ELECTROH CORAELATIONS IN SOLID STATE PHYSICS*

\author{
James Knox Freericks \\ Department of Physics \\ Univergity of California \\ Berkeley, CA 94720 \\ and \\ Materials Sciences Division \\ Lawrence Berkeley Laboratory \\ Berkeley, CA. 94720
}

Apri1 1991

*This work was supported in part by the Director, office or Energy Research, office of Basic Energy Sclences, Materials Sciences Diviaton of the U. $S$. Department of Energy under Contract No. DE-AC03-76SF00098. 


\title{
Electron Correlations in Solid State Physics
}

\author{
Copyright (C) 1991
}

by

James Knox Freericks

The U.S. Department of Energy has the right to use this thesis for any purpose whatsoever including the right to reproduce all or any part thereof 


\title{
Electron Correlations in Solid State Physics
}

\author{
By \\ James Knox Freericks
}

\begin{abstract}
Exactly solvabie models of electron correlations in solid state physics are presented. These models include the spisless Falicov-Kimball model, the $t-t^{\prime}-J$ model, and the Hubbard model. The spinless Falicov-Kimball model is analyzed in one-dimension. Perturbation theory and numerical techniques are employed to determine the phase diagram at zero temperature. A fractal structure is found where the ground-state changes (discontinuously) at each rational electron filling. The $t-t^{\prime}-J$ model (strongly interacting limit of a Hubbard model) is studied on eight-site small clusters in the simple-cubic, body-centered-cubic, face-centered-cubic, and square lattices. Symmetry is used to simplify the problem and determine the exact many-body wavefunctions. Ground states are found that exhibit magnetic order or heavyfermionic character. Attempts to extrapolate to the thermodynamic limit are also made. The Hubband model is examined on an eight-site square-lattice cluster in the presence of and in the absence of a "magnetic field" that couples only to orbital motion. A new magnetic phase is discovered for the ordinary Hubbard model at half-
\end{abstract}


filling. In the "magnetic field" case, it is found that the strongly frustrated Heisenberg model may be studied from adiabatic continuation of a tight-binding model (from weak to strong coupling) at one point. The full symmetries of the Hamiltonian are urilized to make the exact diagonalization feasible. Finally, the presence of "hidden" extra symmetry for finite size clusters with periodic boundary conditions is analyzed for a variery of clusters. Moderately sized systems allow nonrigid tansformarions that map a lattice onto itself preserving its neighbor structure; similar operations are not present in smaller or larger systems. The additional symmetry requires parricular representajons of the space group to stick together explaining many puzzling degeneracies found in exact diagonalization studies. 
This thesis is dedicated to my father, Charles Knox Freericks 


\section{Table of Contents}

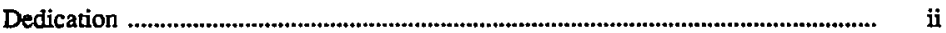

Table of Contents ......................................................................................................... iji

Acknowledgments ....................................................................................................... vi

Chapter I: Introduction ................................................................................................... I

I.1 Overview of the Many-Body Problem ...........................................................

1.2 Models of Electron Correlation .....................................................................

1.3 Methods of Solution .....................................................................................

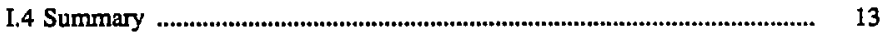

I.5 Appendix: Algorithms for Exact-Diagonalization Studies ......................... 16

I.6 References for Chapter I .................................................................. 19

I.7 Figures for Chapter I ............................................................................... 23

Chapter II: The One-Dimersional Spiniess Falicov-Kimball Model ...................... 25

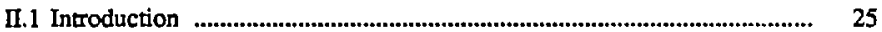

II.2 Perturbative Analysis ........................................................................... 30

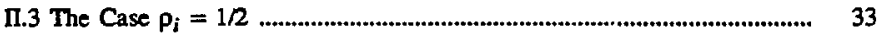

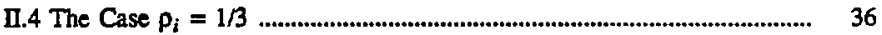

II.5 Conclusion ................................................................................................. 37

II.6 Appendix: Proof of the Periodic Ground-State Theorems ..................... 38

II.7 References for Chapter II ...................................................................... 41

II.8 Tables for Chapter II ................................................................................. 42

II.9 Figures for Chapter II ......................................................................... 
Chapter III: Exact Solution of the $t-t-J$ Model on Eight-Site Cubic Systems

III.1 Introduction ......................................................................................... 58

III.2 Calculational Details .................................................................................. 62

III.3 Results: Ground-State Symmetry ...................................................... 65

III.4 Results: Heavy-Fermion Behavior ........................................................... 71

III.5 Conclusions ............................................................................................... 73

III.6 Appendix: Space-Group Symmetry of Eight-Site Clusters ..................... 75

III.7 References for Chapter III .................................................................. 77

III.8 Tables for Chapter III ........................................................................ 83

III.9 Figures for Chapter III .............................................................. 100

Chapter IV: Exact Solutions of Frustrated Ordinary and Chiral Eight-Site

Hubbard Models

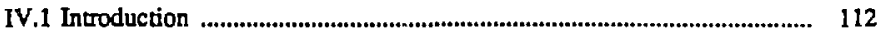

IV.2 Symmetries and Calculational Methods ............................................. 118

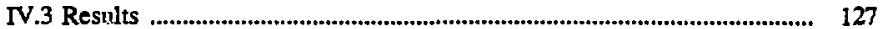

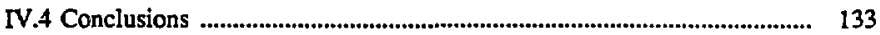

IV.5 Appendix: Full Gauge-Space Group _.................................................... 135

IV.6 References for Chapter IV ............................................................. 138

IV.7 Tables for Chapter IV ........................................................................

IV.8 Figures for Chapter IV ..................................................................... 157

Chapter V: Enlarged Symmetry Groups of Finite-Size Clusters with Periodic Boundary Conditions ..................................................................................................... 168

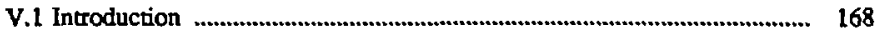


У.2 Group Theory for Cubic Clusters ............................................................ 170

V.3 Example: The Square Lattice ................................................................ 173

V.4 Eight-Site Clusters and the $t-t^{\prime}-J$ Model ......................................... 175

V.5 Conclusions ................................................................................................... 178

V.6 Appendix: Linear-Pair Rule ..................................................................... 179

V.7 References for Chapter V ......................................................................... 181

V.8 Tables for Chapter V ........................................................................ 184

V.9 Figures for Chapter V ........................................................................... 194 


\section{Acknowledgments}

The six years I have spent in Berkeley have been an enjoyable and enlightening experience for me. There are many people I would like to thank who are not explicitly mentioned below. I am grateful to them all.

I would first like to thank my thesis advisor Leo Falicov. He gave me an opportunity to switch fields into condensed matter theory when I was struggling to free myself from "advisor Hell." His guidance, insight, and friendship have provided me with a perfect environment for pursuing academic research. Working with him has been one of my most rewarding experiences.

I would like to thank the members of the Falicov greup who have provided a dynamic atmosphere for research and who have aided me with many stimulating discussions. I would like to thank Dr. Ariel Reich, Dr. Daryl Chrzan, Amitesh Mait, Randolph Hood, Antonio Silva. Dr. Cesar Prortto, Dr. Changfeng Chen, Dr. Henrik Svensmark, Dr. Andreas Giesekus, and Dr. Emilio Artacho.

I would also like to thank the other members of the condensed matter theory group at Berkeley: Prof. Marvin Cohen; Prof. Steven Louie; Prof. Dan Rokhsar, Dr. Troy Barbee; Alberto Garcia; Michael Surh; Xuejun Zhu; and those not explicitly mentioned.

I would like to thank my friends and roommates who have maintained the spirit and vitality of our undergraduate days and have shown me how to balance work in Physics with life outside of Physics.

Finally, I would like to thank my family who made this all possible: my mother whose love and support have helped me endure the hard times and enjoy the good times; my father whose understanding, kindness, and belief in family will never be forgotten; and my brother whose love and friendship have always been present. 


\section{Chapter I: Introduction}

\section{I.1 Overview of the Many-Body Problem}

The many-body probleun constitutes the quantum-mechanical problem of a system with a large number of mutually interacting particles. Its solutions are rich in structure and complex, having applications to many areas in physics and chemistry. The essence of the many-body problem involves the study of correlated motions of particles; i.e., the response of the other particles in the system to the motion of a given particle.

There are many examples of physical phenomena that originate from the correlated motion of electrons. The simplest example is of Van der Waals forees berween neutral atoms. When two atoms are brought close together, the electronic charge cloud of one atom interacts with the charge cloud of the other so that the electrons avoid each other. This correlated motion of the electrons produces fluctuating dipole moments on each atom that attract each other with a potential that depends on the inverse sixth power of the distance between the atoms. Another example is an exciton in a semiconductor. An exciton is formed by the cerrelated motion of an electron in a conduction band and a hole in the valence band that allow the particles to bind together into a complex. A third example is that of a (Mott-Anderson) insulator. Consider a crystal composed of ions that have one conduction elecuron per site and a strong electron-electron interaction that forbids more than one electron on any ion at any time. Band theory (which neglects the electron-electron interaction) predicts that the material is a metal with a half-filled conduction band. When electron currelations are taken into account, the material is a perfect insulator, since the electrons are "frozen" at each lattice site.

Approximation methods must be used, in general, to try to understand the manybody problem. The simplest approximation is to assume the particles are independent 
of one another (although they may interact with a common exuemal potential), the independent-particle (or single-particle) picture. Residual particle-particle interactions may then be added in a self-consistent manner. The independent-particle model neglects correlations between particles, but can be explicitly solved. The problem of treating particle correlations on the same footing as the excernal interactions is a difficult one that has occupied physicists for more than 50 years.

An analogy to the difference in difficulty between the single-particle appreach and the full many-hody problem can be made with two childhood puzzles: the square alphabet puzzle and Rubik's cube. The object of the square alphabet puzzle is to arrange 24 interlocked plastic tiles, each with a letter of the alphabet on it, into alphabetic order on a $5 \times 5$ square. The Rubik's cube puzzle has a $3 \times 3 \times 3$ cube, with each elementary square face colored in one of six colors, that must be rearranged so that each side of the full cube is one color. There are two methods to use in solving the problern. The first method is analogous to the independent-particle picture. The puzzle is disassembled, the pieces arie sorted, and the puzzle is reassembled in solved form. In this solution, each piece of the puzzle is teared independently and the only difficulty is figuring out how to take the puzzle apart and put it back together again. The second method is analogous to the full many-body problem. One figures out how to move individual pieces of the puzzle from one spot to another, taking into account the "correlated" motion of all the other pieces, in such a fashion as to completely solve the puzzle. Although both methods yield the same final result, the first method is viewed as a cheat and the second as an elegant solution (even though it is much more difficult). The many-body problem is not as cut and dry as this example suggests the independent-particle solution usually does not agree with the full many-body solution (due to correlation effects) and the determination of the full many-body solution can be prohibitively more difficult than the indepencent-particle solution. 


\section{I.2 Models of Electron Correlation}

One of the most popular models for strong electron comrelations in solid state physics is the Hubbard model ${ }^{1}$ and its approximations. Originally introduced in the early 1960's, the Hubbard model corsists of two interactions: a tight-binding (hopping) term that models the kinetic energy in a solid and a shor-range (0.1-site) Coulenb interaction term that models the screered electron-electron repulsion in a metal. Th: single-band Hubbard Hamiltonian is

$$
H_{H \times b b}=-\sum_{i, j ; \sigma} t_{i j \sigma} c_{i \sigma}^{t} c_{j \sigma}+U \sum_{i} r_{i \downarrow} n_{i \uparrow},
$$

where $c_{i \sigma}^{\dagger}\left[c_{i \sigma}\right]$ creates [annihilates] a localized (Wannier) electron, with $z$-component of spin $\sigma$, at site $i, n_{i \sigma}=c_{i \sigma}^{\dagger} c_{i \sigma}$ is the corresponding number operator, $t_{i j \sigma}$ is the tight-binding (hopping) matrix, and $U$ is the on-site Coulomb interaction.

The Hubbard model (and its multi-band generalization) was introduced to describe electronic systems in narrow-band materials such as transition metals. It has a deceptively simple Hamiltonian constructed from two noncommuting terms that can be explicitly diagonalized separately (in momentum space for the hopping term) but defy general solution when combined together. The model has been solved exactly in one-dimension using the Bethe ansatz by Lieb and Wu. ${ }^{2}$ However, the wavefunctions are so complex that, even after twenty years of research, their properties have not been completely determined ${ }^{3}$ The Hubbard model is diagonalized exactly for an eight-site system in Chapter IV.

There is an interesting map of the many-body Hubbard Hamiltonian (1.1) onto an effective tight-binding model that is noninteracting ${ }^{4}$ (see below for an example). The "latrice" points denote each of the linearly independent basis states of the many-body Hilbert space. The "lattice connectivity" is determined by how each state is mapped into one another by the Hubbard Hamiltonian. The result is an effective tight-binding model (including on-site terms) that can be trivially diagonalized in theory. In 
practice, the problem becomes intractable for a large system since the effective lattice does not have simple periodic symmetry. In other words, the mapping to an effective lattice model indicates that the Hubbard Hamiltonian matrix is a large $\left[\exp \left(10^{23}\right) \times \exp \left(10^{23}\right)\right]$ and sparse (only many-body states with $N-1, N$, or $N+1$ doubly occupied sites are coupled to a state with $N$ doubly occupied sites) matrix but it cannot be reduced to a very small matrix by symmetry aione (as is done in the phonon problem with the dynamical matrix and Bloch's theorem).

There are two approximations to the Hubbard Hamiltonian shat are commonly studied: the spiniess Falicov-Kimball model ${ }^{5}$ and the $t-J$ model. ${ }^{6}$ Both approximations arise from different limiting forms of (1.1).

The spinless Falicov-Kimball model ${ }^{5}$ results when the Hubbard Hamiltonian is generalized to have spin-dependent hopping $\left(i_{i j} \uparrow \neq t_{i j \downarrow}\right)$ and the limit of infinitely massive spiii-down electrons is taken $\left(t_{i j \downarrow} \rightarrow 0\right)$. The spin-up electrons are independent of one another and have an on-site interaction with the spin-down electrons that are frozen in a particular configuration on the lattice. If a classical variable $W_{i}$ is defined to be one [zero] if the $i$ th site is occupied [unoccupied] by a spin-down electron, then the spinless Falicov-Kimball model is described by the following Hamiltonian

$$
H_{F-K}=-\sum_{i, j} t_{i j} c_{i}^{\dagger} c_{j}+U \sum_{i} n_{i} W_{i}
$$

where the (up) spin subscript has been dropped for convenience. The many-body physics enters by a minimization process where the ground-state configuration $\left(W_{i}\right)$ is determined as the configuration (with fixed static-particle number) that minimizes the total energy of the itinerant particles. This model has many different physical interpretations. It was originally introduced ${ }^{5}$ to describe metal-insulator transitions in rareearh and transition-metal compounds. The itinerant particles correspond to conduction electrons and the static particles to the localized ( $d$ - or $f \rightarrow$ ) electrons. It has subsequently been used to describe electron-induced crystallization ${ }^{7}$ where the static 
particles are now interpreted as ions. The one-dimensional version of the spinless Falicov-Kimball model is studied in Chapter II.

The $t-J$ model $^{6}$ is the strong-coupling limit $(U \rightarrow \infty)$ of the Hubbard model. The full Hilber space of the Hubbard model is truncated to a Hilbert space that does not include any of the many-body states that have more than one electron zar site. In other words, the electrons satisfy a "super" Pauli principle that forbids two electrons with the same spin or opposite spin from occupying the same localized orbital. Residual interactions arise from Anderson's superexchange ${ }^{6}$ and correspond to a nearestneighbor Heisenberg antiferromagnet. The $t-J$ Hamiltonian is derived from a cancnical transformation ${ }^{8.9}$ of (1.1) that projects out doubly occupied states. The result is (to second order)

$$
H_{t-J}=-\sum_{i, j ; \sigma} t_{i j}\left(1-n_{i-\sigma}\right) c_{i \sigma}^{\dagger} c_{j \sigma}\left(1-n_{j-\sigma}\right)+\sum_{i, j} J_{i j} \mathbf{s}_{i} \cdot \mathbf{s}_{j},
$$

when three-site interactions are neglected. The $t-J$ model is examined for a variety of geometries in Chapter III.

In order to make contact with macroscopic (real) systems, the many-body problem must be solved in the thermodynamic limit (number of particles $\rightarrow \infty$ ). This is not possible in general, so approximation schemes must be undertaken. The approximation schemes (see below) fall into two broad categories - the perturbation approach $^{10}$ and the small-cluster approach. ${ }^{11}$ The perturbation approach takes the thermodynamic limit of an independent-particle system and then treats the interactions (usually in an approximate fashion). This first approach depends on the applicability of adiabatic continuation: as the interactions are slowly turneo on, the ground state evolves from an independent-particle ground state to the many-body ground state in a smooth fashion without passing through any phase boundaries. The small-cluster approach stans with a finite system and solves (exactly or approximately) for the many-body solution. These solutions are then extrapolated for larger and larger 
systems to reach the thermodynamic limit. This second approach is subject to many uncontrollable finite-size effects that make the extrapolation process very dificult.

The difference between these two approaches lies in the different order that limits are taken. The perturbation approach works with a large number of particles then turns on the particle-particle interaction, whereas the small-cluster approach first turns on the particle-particle interactions and then lets the system grow. In an ideal siruation, both approaches would be ey licitly solvable and the two solutions would agree (indicating that the order of taking limits is not important). However, whether or not this holds in general is unknown, since neither means of solution can be carried out, for most systems, to suitable limits.

Electro:s in metals form an example to the perturbation approach. ${ }^{10}$ In the independent-particle (one-electron) picture, the electrons fill every one-electron level of the metallic band structure that lie below the Fermi energy. The Fermi surface is the (multiply) connected surface that separates, in $k$-space, occupied and unnccupied states. The electron filling is a step function in one-electron-energy space (see Fig. 1.1). When interactions between the electrons are slowly turned on, the electron filling is modified, with some previously occupied states being emptied and some previously empty states being filled. If perturbation theory is valid; ${ }^{12}$ i.e., the many-body ground state is adiabatically connected to the one-electron ground state, then the distribution is changed (see Fig. 1.1) but a discontinuity in $k$-space still exists that defines the Fermi surface. The volume enclosed by the Fermi surface, before and after the interactions are tumed on, is unchanged. Elementary excitations (quasi-electrons, quasi-holes) are well-defined near the Fermi surface. These excitations are dressed electrons and holes that correspond to a bare (real) particle clothed by its screening cloud. The quasiparticles have very small residual interactions between one another and are well-defined because they have long lifetimes. The analysis of this quasiparticle system is called Fermi liquid theory and was originally proposed by Landau. ${ }^{13}$ 
The two-site Hubbard model (Hydrogen molecule) is the simplest example of the small-cluster approach. It was solved independently by Harris and Lange ${ }^{8}$ and Falicov and Harris. ${ }^{14}$ It corresponds to the Hubbard Hamiltonian (1.1) with $i$ and $j$ running over 1 and 2 only and $t_{12 \sigma}=t_{21 \sigma}=t$. The one and three electron problems are identical to the independent-particle solution so only the half-filled case of two electrons is considered. The Hamiltonian is spin-rotationally symmetric so the many-body eigenstates can be classified according to their total spin ( $S=0$ or 1). Without loss of generality, the problem can be restricted to $S_{Z}=0$. There are four many-body eigenstates:

$$
\begin{aligned}
& 11>=c \uparrow_{\uparrow} \ddagger_{\downarrow} 10>=\uparrow \downarrow 0 \quad ; \\
& 12>=c_{2 \uparrow}^{\dagger} \uparrow \downarrow_{\downarrow} 10>=0 \uparrow \downarrow ； \\
& 13>=c_{1} c_{2} t_{\downarrow} 10>=\uparrow \quad \downarrow ; \\
& 14>=c_{2} \uparrow c t \downarrow \mid 0>=\downarrow \uparrow .
\end{aligned}
$$

The effecrive tight-binding model ${ }^{4}$ is illustrated schemarically in Fig. 1.2.

The spin symmetry has not been taken into account yet. Neither has the spatial symmetry $P$ that permutes sites 1 and 2 . Wavefunctions can be classified as being even or odd under the operation $P$. Changing basis functions to those that have definite spin and spatial symmetry produces

$$
\begin{aligned}
& \left.\mid a>=\frac{1}{\sqrt{2}}(|1>+| 2\rangle\right), \text { even, } s=0, \\
& \mid b>=\frac{1}{\sqrt{2}}(|1>-| 2>), \text { odd, } s=0, \\
& \mid c>=\frac{1}{\sqrt{2}}(|3>+| 4>), \text { even, } s=0, \\
& \mid d>=\frac{1}{\sqrt{2}}(|3>-| 4>), \text { odd, } s=1 .
\end{aligned}
$$

Nore that the assignment of spin to the states $|c\rangle$ and $|d\rangle$ is not incorrect. It arises 
from the convention of labeling many-body states. In particular, if the spin-lowering operator is applied to the $S=1, S_{Z}=1$ eigenstate

$$
\begin{aligned}
& \left.s_{-} c_{1 \uparrow} c_{2 \uparrow}^{\dagger} 10>=\left(c_{1} c_{1 \uparrow}+c_{2 \downarrow}^{\dagger} c_{2 \uparrow}\right) c t_{\uparrow} c_{2 \uparrow}^{\dagger} 10\right\rangle \\
& =|3\rangle-|4\rangle=\sqrt{2}|d\rangle,
\end{aligned}
$$

then the $S=1, S_{Z}=0$ eigenstate results, as claimed. The only states that can be mixed by the Hamiltonian are $|a\rangle$ and $|c\rangle(|b\rangle$ and $|d\rangle$ are eigenstates with eigenvalues $U$ and 0 respectively). The reduced Hamiltonian block is

$$
\left[\begin{array}{cc}
U & -2 t \\
-2 t & 0
\end{array}\right]
$$

for the $|a\rangle,|c\rangle$ subspace with eigenvalues

$$
E_{ \pm}=\frac{U}{2} \pm \frac{1}{2} \sqrt{U^{2}+16 t^{2}}
$$

The ground state has energy $E_{-}$, zero spin, and is even under $P$. Note that by taking full advantage of the symmetry, the problem was reduced to a trivial one.

\section{I.3 Methods of Solution}

There are many different techniques that have been applied to find exact or approximate solutions to the many-body problem. A comprehensive (but not exhaustive) list is given below of the different methods that have been applied to Fermi systems in solid-state physics. The techniques have been grouped into categories that denote the types of methods that are used. These designations are somewhat arbitrary and are not exclusive, as some techniques fall into more than one caregory.

(i) Direct perturbative methods

Perturbation theory can take on many different forms but all methods are based on the assumption that interactions are "small" in comparison to the unperturbed system. Direct perturbation theory ${ }^{15}$ (Rayleigh-Schßdinger perturbation theory) has 
notorious difficulties when applied to the many-body problem ${ }^{10}$ with calculated results diverging to ail orders (this arises in part because the perturbation series tends to be an asymptotic series rather than a Taylor series). Wigner-Brillouin perturbation theory ${ }^{16}$ produces energies that are solutions of algebraic equations but it generally yields results that are less accurate than the Rayleigh-Schrödinger series.

One promising method produces eigenvalue expansions in the form of continued fractions (a special case of Padé approximations) and is called the projection method. ${ }^{17}$ It is based on similar techniques used in statistical mechanics called the memoryfunction formalism. ${ }^{18}$ High-order expansions are difficult because they involve complicated cumulant averages of operators, but low-order results produce quite accurate energies.

(ii) Diagrammatic methods

Diagrammatic methods use powerful techniques of parially summing infinite series of diagrams (corresponding to infinite-order summations of perturbation series) to eliminate spurious divergences of direct perturbation theory. Diagrammatic expansions are based strongly on the concept of adiabatic (analytic) continuation from a noninteracting system to a strongly interacting system (i.e., the perturbation series can be summed and analytically continued if handled "properly"). Dyson's equation "s is the principle means used to extract information from the diagrammatic expansion. To illustrate consider the geometric series $F(x)$

$$
F(x)=\sum_{n=0}^{\infty} x^{n}=1+x F(x) .
$$

The secend equality is the simplest form of a Dyson equation that can be solved $F(x)=1 /(1-x)$. The power series only converges for $|x|<1$, but the Dyson equation produces the correct analytic continuation of the power series for all $x \neq 1$.

Diagrammatic methods have been quite successful in a low-density Fermi sys$\operatorname{tem}^{19}$ (the ladder approximation) and in the high-density Fermi system ${ }^{20}$ (the random- 
phase approximation). These methods can be pushed further by self-consistently renormalizing the diagrams ${ }^{10}$ to calculate the residual interactions between dressed particles.

(iii) Uncontrolled methods

Uncontrolled methods are all based on approximations that have no internal test for how important a role the neglected terms make to the solution. The variational method is one of the most popular uncontrolled approximations. In the variational approach the many-body ground state is approximated by a trial stave which produces an upper bound to the ground-state energy. Unfortunately, the variational method is more of an art than a science, since the end results are only as good as the input trial states.

The simplest variational approximation is the Hartree-Fock approximation ${ }^{21}$ whose trial state is a Slater determinant of independent-particle wavefunctions. This variational calculation can be viewed as a mean-field thcory in the following sense: each particle interacts (self-consistently) with the average field of the other particles and particle-particle correlations are neglected. The Bardeen-Cooper-Schrieffer theory ${ }^{22}$ of superconductivity is another variational method that is a mean-field theory. It agrees extremely well with experimental results for conventional superconductors. Other variational approaches include projecive methods (such as the Gutzwiller ${ }^{23}$ approach) that create trial variational states by continuously interpolating between wavefunctions that satisfy the Pauli principle and wavefunctions that satisfy the super Pauli principle. Laughlin's theory for the fractional quantum Hall effect ${ }^{24}$ illustrates the "artistic" side to the variational method: the trial state is a blind guess that produces remarkable agreement with experiment.

Slave-boson and slave-fermion theories ${ }^{2 x}$ are mean-field theories that have been a popular approach to the strongly coupled limit of the Hubbard model. Auxiliary bosonic or fermionic fields are introduced to enforce the consraint of no double 
occupation of sites (as required by the $t-J$ model) but all particle-particle correlations are neglected.

Density functional theory ${ }^{26}$ (and the more recent quantum Monte Carlo methods ${ }^{27}$ ) have proven themselves as powerful approximations for calculating properties of real materials. The local-density-approximation ${ }^{2 \beta}$ to density-functional theory is an uncontrolled approximation that replaces a functional of the electron density by a function of the electron density, yet it is the state-of-the-art method for band structure calculations that produces remarkable results when combined with the so-called GW approximation ${ }^{29}$ for the quasiparticle excitations.

Other uncontrolled approximations introduce large dimensionalities (in real or internal space) and expand solutions around the infinite-dimensional result. The large$N$ method $^{30}$ for spin systems replaces $S U(2)$ spin operators by $S U(N)$ operators in the limit $N \rightarrow \infty$ and calculates corrections to onder $1 / N$. The large- $D$ method ${ }^{31}$ for strongly coupled particles on a lattice replaces a three-dimensional lattice by a $D$ dimensional lattice in the limit $D \rightarrow \infty$ and calculates corrections to order $1 / D$. Both of these methods produce promising results but it is unclear if the expansions are valid for small (physical) dimensions.

(iv) Phenomenological methods

Fermi liquid theory ${ }^{13}$ is the best example of a phenomenological theory that assumes quasiparticles exist and bave weak residual interactions. Physical phenomena can then be predicted for a wide variery of experiments once a few phenomenological parameters are determined. Phenomenological methods can have nice predictive powers for specific experiments, but they lack direction for insight into microscopic mechanisms, or power of calculation of the parameters that define them.

(v) Direct methods

Direct methods are based on direct manipulation of the many-body Hamiltonian. The equation-of-motion technique ${ }^{10,20}$ calculates the time-evolution of operators from 
the canonical equations of motion

$$
i \hbar \frac{d}{d t} o_{o p}=\left[t_{i}, O_{o p}\right]
$$

These equations frequently link together an infinite number of operators in a chain of coupled differential equations. The standard solution-technique is to decouple the infinite chain after a finite length and explicitly solve the reduced problem.

Another direct method is that of Monte Carlo simulations. ${ }^{32}$ Moderately sized systems are set up with random initial conditions and allowed to evolve in time (under the action of the Hamiltonian) to their ground state. Finite-size effects enter in an uncontrolled fastion and simulations are also bampered by slow evolutions to the ground state.

(vi) Exact solution methods

Exact solutions are the most powerful technique (since no approximations are made) but they rarely occur in the thermodynamic limit. The most common problems that are exactly solvable are one-dimensional or quasi-one-dimensional systems. A Bethe lattice (Cayley tree) is an example of a quasi-one-dimensional system: it is a tree where each node has $z$ branches extending out of it It resembles higherdimensional structures since the coordination number of each lattice site is larger than two, but thete are no closed loops and the surface-to-volume ratio does not vanish in the limit of an infinite lattice. Exact solutions require the use of the Bethe ansatz, 33 or the renormalized-perturbation-expansion ${ }^{34}$ for Green's function. The latter method is used in Chapter II.

Another class of many-body Hamiltonians that can be exactly solved is the class whose Hamiltonian has at most terms that are quadratic in the creation and annihilation operators. Canonical transformations can always be found that diagonalize the Hamiltonian in this case. 
Exact solutions are also possible by direct diagonalization of the many-body problem on small systems - the so-called small-cluster technique. ${ }^{11}$ This method suffers from uncontrolled finite-size effects and cannot be extrapolated to the thermodynamic limit in any obvious way. The small-cluster techrique is used in Chapters III and IV. The appendix includes a detailed description of the algorithms used to incorporate the full symmetry group of the Hamiltonian in its exact diagonalization.

\section{I.4 Summary}

Analysis of the symmetries and invariances of the many-body Hamiltonian is a very poweriul weapon in the many-body physicists arsenal. The consequences of the symmetry group of the Hamiltonian are rigorous, exact results that do not ciepend on any approximation scheme. Symmetry is also used to reduce a large (intractable) problem to a smaller (manageable) one. This contribution utilizes the full symmetry of the Hamiltonian as a guiding principle to determine the exact solution of model many-body problems.

The mathematical models are abstracted from physical systems and simplified in order to make it feasible to find their exact solution. The hope is that the essence of the underlying physicai mechanisms (that determine the strongly interacting phenomena in real materials) are captured by these simplified models. In this sense, the microscopic physics can be qualitatively understood by studying the many-body solutions of the abstract models.

Why do most solids crystallize in a periodic structure and what role do particleparticle correlations play in determining these periodic structures? This is one of the most fundamental questions of solid state physics that remains unanswered. There are many other related questions in the context of the alloy problem; e.g., why do certain combinations of elements form ordered solid. solutions, others form solutions that are disordercd, and still others not form solutions at all? It is very difficult to incorporate 
the quantum-mechanical problem of solu'bility with the thermodynamic problem of ordering and include many-body interactions into one self-consistent theory.

Chapter II of this contribution makes inroads into these questions of lattice formation and stable alloy phase formation for a very simple model ${ }^{35}$ - the onedirnensional version of the Falicov-Kimball model for spinless electrons (1.2). This model describes a one-dimensional crystal or, equivalently, a one-dimensional binary alloy. Many-body interactions play a crucial role, determining periodic latticestructures or segregated alloy phases, as a function of electron concentration and electron-ion interaction strength. The renormalized-perturbation-expansion is used to find the exact ground-state energies in the thermodynamic limit. Phase diagrams are calculated for specific examples. The numerical results are extrapolated to qualitatively determine the complete solution.

Chapter III discusses the strongly interacting limit of the Hubbard model (the $t-J$ model) on eight-site small clusters with cubic [and square] geometries. ${ }^{36}$ The $t-J$ model (1.3) is used to describe materials that sustain long-range magnetic order or heavy-fermionic behavior. Transition metals $(\mathrm{Fe}, \mathrm{Co}, \mathrm{Ni})$ form itinerant ferromagnets; i.e., the magnetism arises from mobile electrons and their correlations with one another. Transition-metal oxides and halides $\left(\mathrm{NiO}, \mathrm{Ni}_{2}\right)$ tend to be antiferromagnetic (Mott-Anderson) insulators. Lanthanide or actinide compounds $\left(\mathrm{CeCu}_{6}, \mathrm{UPt}_{3}, \mathrm{UBe}_{13}\right)$ display physical properies of a system with a huge electronic effective mass (large density-of-states at the Fermi level). These so-called heavy-fermion materials have gigantic coefficients to the linear term in the specific heat, large magnetic susceptibilities, (poor) metallic conductivity, and may become superconducting. The $t-J$ model includes many-body solutions that exhibit (qualitatively) the same physical phenomena. The small-cluster approach is used to exactly diagonalize the $t-J$ model for a variety of different systems and determine how important a role geometry $2: \leq \mathrm{s}$ electron correlation plays in determining these properties. 
In Chapter IV, some aspects of the resonating-valence-bond model of hightemperature superconductivity are studied for (generalized) Hubband models in ficticious magnetic fields ${ }^{37}$ (that couple only to orbital inotion). The premise of these theories is that the half-filled band Mott-insulating state does not sustain long-range order but is "liquid-like" with short-range antiferromagnetic correlations. The superconducting state arises when the system is doped to include charge carriers. The Hubbard Hamiltonian is exactly diagonalized for an eight-site square-lattice cluster (that approximates the $\mathrm{CuO}_{2}$ planes in the new supercenductors) and the applicability of adiabatic continuation from weak-coupling to strong-coupling is analyzed. It is found that a strongly frustrated Heisenberg antiferromagnet can be studied as the Gutzwillerprojected limit of a tight-binding model at only one point in the phase diagram (in a suitable magnetic field). It is unknown whether ( $\tau$ not this phenomenon survives in the thermodynamic limit. A novel mechanism for producing (macroscopic) magnetic moments in a half-filled band is also discovered for a strongly frustrated system.

The final chapter describes hidden symmetries of finite clusters with periodic boundary conditions. ${ }^{38}$ The transition from a self-contained (isolated) cluster to an infinite lattice passes through an intermediate region where the system has extra symmetry. These extra symmetry operations correspond to nonrigid transformations that map the lattice onto itself. The presence of this additional symmetry produces sticking together of many-body energy levels (that have different spatial symmesries) thereby explaining many puzzling degeneracies found in exact-diagonalization studies. The presence of a large symmetry group allows some moderately sized many-body Hamiltonia to be analyrically diagonalized. 


\section{I.5 Appendix: Algorithms for Exact-Diagonalization Studies}

The incorporation of symmetry and the calculation of reduced Hamiltonian blocks for the Hubbard model (and its generalizations) is a straightforward process that rapidly becomes tedious. Computer algorithms are utilized to perform this algebraic task exactly to prevent errors and to make calculations on larger systems feasible. A set of powerful FORTRAN codes have been written to calculate the Hamiltonian in (symmetrized) block-diagonal form for the single-band Hubbard model or the $t-J$ model on arbitrary lattices (with a size of up to 16 sites). Some of the codes are based upon the work ${ }^{39}$ of Ariel Reich.

The matrix-element theorem ${ }^{40}$ (generalized Unsbld theorem) guarantees that a Hamiltonian is in block-diagonal form (with no mixing between blocks that have different symmery) when it is expanded in a coordinate basis consisting of functions that have definite symmetry. Two types of symmetry are taken into account: spacial symmetry and spin symmerry. Basis functions with definite symmetry transform as the (1,1) matrix elements of an irreducible representation of the cluster-permutation group (see Chapter V) and form spin-multiplets (with $m_{S}=S$ ). Projection operators are used on a "seed" state to produce the functions with definite symmetry.

The spin symmetry is constructed in a brute-force fashion. A bootstrap process is employed, wherein total-spin eigenvectors for $N+1$ electrons are built from the eigenstates for $N$ electrons, according to the following rules (a state denoted by $\left|S, m_{S}\right\rangle$ has total spin $S$ and total $z$-component of spin $m_{S}$ ):

$$
\begin{aligned}
& \left|s+\frac{1}{2}, s+\frac{1}{2}>=\right| s, s>\otimes \mid \uparrow>, \\
& \left|s-\frac{1}{2}, s-\frac{1}{2}>=\frac{1}{\sqrt{2 S+1}}\right| s, s-1>\otimes\left|\uparrow>-\frac{2 s}{\sqrt{2 S+1}}\right| s, s>\otimes \mid \downarrow>, \\
& \left.\left|s-\frac{1}{2}, s-\frac{1}{2}>\propto[s-\mid s, s>] \otimes\right| \uparrow>-2 s|s, s>\otimes| \downarrow>\quad, \quad 1.11\right)
\end{aligned}
$$

where $S_{-}$is the total-spin lowering operator 


$$
s_{-}\left|s, m_{S}>=\hbar \sqrt{\left(S+m_{S}\right)\left(S-m_{S}+1\right)}\right| s, m_{S}-1>\text {, }
$$

and the last line (unnormalized state) shows that all of the coefficients can be stored as integers and be normalized later. The recursion starts with the spin-up state $1 \frac{1}{2}, \frac{1}{2}>=1 \uparrow>$.

This method of generating total-spin eigenstates in a localized basis is cumbersome for a large number of particles. The :nemory space required for 16 particles is prohibitively large. A better method has been recently suggested ${ }^{41}$ by R. Saito (but has not been incorporated yet into the routines): the total-spin eigenstates can he stored in a dimer notation with each dimer corresponding to a spin-singlet or a spintripiet pair. The dimers are arranged in the form of noncrossed bond diagrams according to the Young tableau (permutation-symmetry representations) corresponding to a total-spin eigenstate (the Young tableaux contain one of two rows with the total spin equal to half the number of columins in the first row minus half the number of columns in the second row). This procedure is a much more compact method for generating and storing total-spin eigenstates.

The spadial projection operators are also easily generated. They are constructed from the $(1,1)$ matrix elements of each irreducible repressntation of the clusterpermutation group in the standard fashion. ${ }^{42}$ Computer codes are available to determine the different irreducible representation marrices from the group elements and the character table.

Projection operators are used to construct symmetrized basis states with definite total spin, maximal $z$-cemponent of spin $\left(m_{S}=S\right.$ ), and with definite spatial symmerry. The Hamiltonian subblocks for each symmetry subspace are then constructed. The block-diagonal Hamiltonian is checked for completeness and hermiticity. The algebraic operations are performed exactly using integer arithmetic throughout (including multiple-precision integer arithmetic ${ }^{43}$ when required). It is found that the 
symmetriztd Hamiltonian blocks remain quite sparse in general.

The Hamiltonian blocks are diagonalized by the so-called QL algorithm ${ }^{44}$ for block sizes smaller than $100 \times 100$ and by the Lanczos method ${ }^{45}$ for larger block sizes. All of the eigenvectors and eigenvalues can be determined, if desired. Correlation functions (operator ground-state averages) can also be calculated. A correlationfunction matrix is generated for the symmetrized subspace that includes the ground state. The expectation value is determined by double-contracting the ground-state eigenvector with the correlation-function matrix.

Further details may be found in the documentation that accompanies the computer codes (available from the author upon request). 


\section{I.6 References for Chapter I}

1 J. Hubbard, Proc. R. Soc. London, Ser. A 276, 238 (1963); ibid., 277, 237 (1964); ibid., 281, 401 (1964); ibid., 285, 542 (1965); ibid., 296, 82 (1967); ibid., 296, 100 (1967).

2 E.H. Lieb and F.Y. Wu, Phys. Rev. Lett. 20, 1445 (1968).

3 M. Ogata and H. Shiba, Phys. Rev. B 41, 2326 (1990); M. Brech, J. Voit, and H. Blitmer, Europhys. Lett. 12, 289 (1990).

S.A. Trugman in Applications of Statistical and Field Theory Methods to Condensed Matter, edited by D. Baeriswyl, A.R. Bishop, and J. Carmelo, NATO ASI Series B, Vol. 218, (Plenum, New York, 1990) p. 253.

L.M. Falicov and J.C. Kimball, Phys. Rev. Lett. 22, 997 (1969).

P.W. Anderson, in Solid State Physics, edited by F. Seitz and D. Turnbull, (Academic, New York, 1963) Vol. 14, p. 99.

U. Brandt and R. Schmidt, Z. Phys. B 63, 45 (1986); ibid., 67, 43 (1987); T. Kennedy and E.H. Lieb, Physica 138A, 320 (1986); E.H. Lieb, ibid., 140A, 240 (1986).

A.B. Harris and R.V. Lange, Phys. Rev. 157, 295 (1967).

A.H. MacDonald, S.M. Girvin, and D. Yoshioka, Phys. Rev. B 37, 9753 (1988); A.M. Oles, îbid., 41, 2562 (1990); A.H. MacDonald, S.M. Girvin, and D. Yoshioka, ibid., 41, 2565 (1990).

D. Pines, The Many-Body Problem (Benjamin, Reading, 1961); A.A. Abrikosov, L.P. Gorkov, and I.E. Dzyaloshinski, Methods of Quantum Field Theory in Staristical Physics (Dover, New York, 1975); R.D. Mattuck, A Guide to Feynman Diagrams in the Many-Body Problem (McGraw-Hill, New York, 1976).

11 L.M. Falicov, in Recent Progress in Many-Body Theories, edited by A.J. Kallio, E. Pajanne, and R.F. Bishop, (Plenum, New York, 1988) Vol. 1, p. 275; J. 
Callaway, Physica B 149, 17 (1988).

A.B. Migdal, Sov. Phys. JETP 5, 333 (1957); W. Kohn and J.M. Lutinger, Phys. Rev. 118, 41 (1960); J.M. Luttinger and J.C. Ward, ibid., 118, 1417 (1960); J.M. Luttinger, ibid., 119, 1153 (1960); J.M. Luttinger, in The Fermi Surface, edited by W. Harrison and M.B. Webb, (Wiley, New York, 1960) p. 2.

L. Landau, Sov. Phys. JETP 3, 920 (1957); ibid., 5, 101 (1957); ibid., 8, 70 (1959).

L.M. Falicov and R.A. Harris, J. Chem. Phys. 51, 3153 (1969).

A. Messiah, Quantum Mechanics, (Wiley, New York, 1958) Vol. 2, Ch. 16.

J.M. Ziman, Elements of Advanced Quantum Theory, (Cambridge University, Cambridge, 1969) p. 53.

K.W. Becker and P. Fulde, Z Phys. B 72, 423 (1988); K.W. Becker, H. Won, and P. Fulde, ibid., 75, 335 (1989); W. Brenig and K.W. Becker, ibid., 76, 473 (1989).

D. Forster, Hydrodynamic Fluctuations, Broken Symmetry, and Correlation Functions, (Benjamin, Reading, 1975) Ch. 5.

V.M. Galitskii, Sov. Phys. JETP 7, 104 (1958).

D. Bohm and D. Pines, Phys. Rev. 92, 609 (1953); M. Gell-Mann and K. Brueckner, Phys. Rev. 106, 364 (1957).

P.W. Anderson, Concepts in Solids, (Addison-Wesley, Redwood City, 1963) Ch. 2.

J. Bardeen, L. Cooper, and J. Schrieffer, Phys. Rev. 108, 1175 (1957).

M.C. Gutzwiller, Phys. Rev. A 137, 1726 (1965).

The Quantum Hall Effect, edited by R.E. Prange and S.M. Girvin (SpringerVerlag, New York, 1987). 
P. Coleman, Phys. Rev. B 29, 3035 (1984).

P. Hohenberg and W. Kohn, Phys. Rev. B 136, 864 (1964).

D. Ceperley and B. Alder, Science, 231, 555 (1986).

W. Kohn and L.J. Sham, Phys. Rev. A 140, 1133 (1965); R.O. Jones and O. Gunarsson, Rev. Mod. Phys. 61, 689 (1989).

L. Hedin and S. Lundqvist, in Advances in Solid State Physics, Vol. 23, edited by F. Seitz, D. Turnbull, and H. Ehrenreich, (Academic, New York, 1969) p. 1.

I. Affleck, Phys. Rev. Lett, 54, 966 (1985); J.B. Marston and I. Affleck, Phys. Rev. B 39, 11,538 (1989).

W. Metzner and D. Vollhardt, Phys. Rev. Lett. 62, 324 (1989).

Monte Carlo Methods in Quantum Problems, edited by M.H. Kalos (Reidel, Dordrecht, 1984).

H.A. Bethe, Z. Phys. 71, 205 (1931); M. Gaudin, Phys. Lett. 24A, 55 (1967); C.N. Yang, Phys. Rev. Lert. 19, 1312 (1967).

P.W. Anderson, Phys. Rev. 109, 1492 (1958); E.N. Economou and M.H. Cohen, Phys. Rev. B 4, 396 (1971); E.N. Economou, Green's Functions in Quantum Physics (Springer-Verlag, Heidelberg, 1983).

J.K. Freericks and L.M. Falicov, Phys. Rev. B 41, 2163 (1990).

J.K. Freericks and L.M. Falicov, Phys. Rev. B 42, 4960 (1990).

J.K, Freericks, L.M. Falicov, and D.S. Rokhsar, unpublished .

J.K. Freericks and L.M. Falicov, unpublished .

Ariel Reich, Ph.D. Thesis, University of California, Berkeley, (1988) unpublished.

M. Tinkham, Group Theory and Quantum Mechanics, (McGraw-Hill, New York, 1964) pp. 20ff and 80ff. 
41 R. Saito, J. Phys. Soc. Japan 59, 482 (1990).

42 L.M. Falicov, Group Theory and Its Physical Applications, (Universiy of Chicago, Chicago, 1966) pp. $57 \mathrm{ff}$.

$43^{\circ}$ R.P. Brent, ACM Trans. on Math. Soft. 4, 57 (1978).

44 J.H. Wilkinson and C. Reinsch, Handbook for Automatic Computation, Vol. II: Linear Algebra, edited by F.L. Bauer, (Springer-Yerlag, New York, 1971) p. 227.

45 J. Cullum and R.A.. Willoughby, Lanczos Algorithms for Large Symmetric Eigenvalue Compurations Vol. 1 and 2 (Birkhauser, Basel, 1985). 


\section{I.7 Figures for Chapter I}

Figure 1.1. Electron filling as a function of one-electron energy for a noninteracting (a) and an interacting (b) electron gas. The horizontal axis records the energy of the one-electron orbital (in arbitrary units) and the vertical axis records the occupation. of the respective orbital. The noninteracting case (a) is a step function with all states occupied whose energy lies below the Fermi level and all states empty whose energy lies above the Fermi level. The interacting case (b) has a modified distribution with some previously occupied states becoming unoccupied and some previously empty states becoming filled. A discontinuity, however, remains to define the Fermi level.
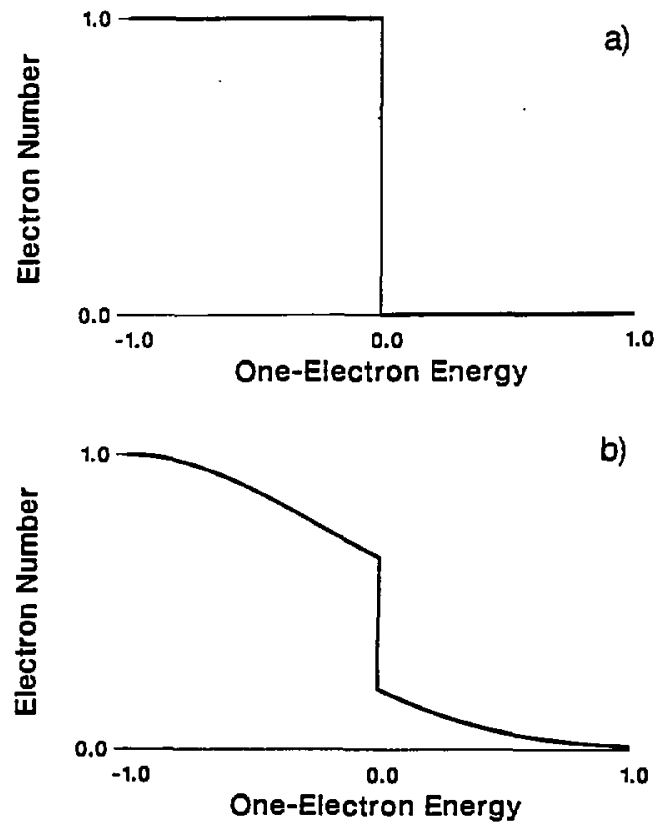
Figure 1.2. Effective tight-binding picture for the two-site Hubbard model. The "lattice sites" correspond to the four many-body states in Eq. (1.4). The "latticeconnectivity" is represented by solid lines corresponding to a hopping integral $(-t)$. The effective tight-binding model also has on-site interactions of size $0[U]$ for the many-body states $\mid 3>$ and $\mid 4>[11>$ and $12>]$.

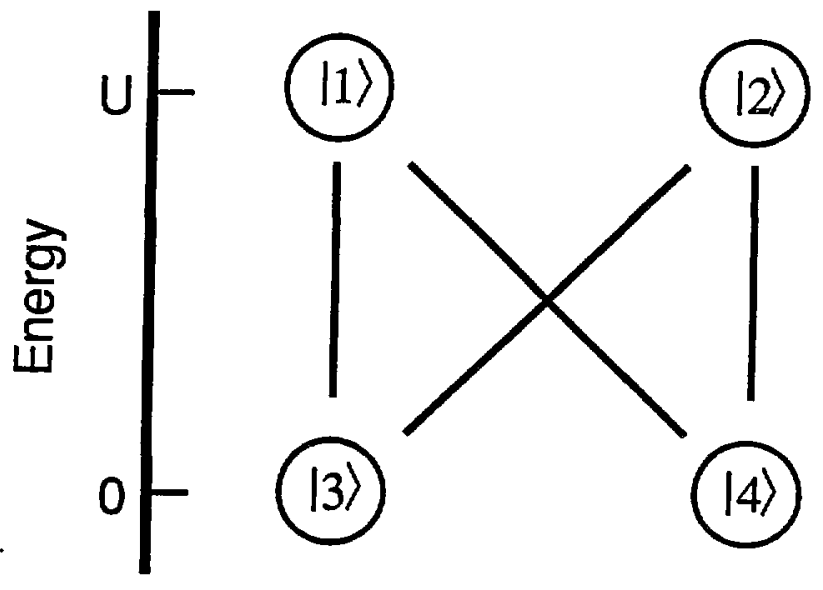




\section{Chapter II: The Ore-Dimensional Spinless Fulicov-Kimball Model}

\section{II.1 Introduction}

It is generally accepted that many properties of heavy-fermion systems and intermediate-valence compounds as well as the phenomena of metal-insulator transitions, itinerant magnetism, metallic crystallization, alloy formation, etc. result from the properties of strongly correlated electrons. There are, however, very few exact results available for correlated electronic systems and approximate methods are sometimes contradictory. In 1969, the Falicov-Kimball model ${ }^{1}$ was introduced as a model for inetal-insulator transitions. It remains one of the simplest interacting fermion systems in which electron correlation effects may be studied exactly. Several rigorous results have already been obtained for the one-band spinless version of the Falicov-Kimball model: Brandt and Schmidt ${ }^{2}$ calculated upper and lower bounds for the ground-state. energy in two dimensions; Kennedy and Lieb ${ }^{3}$ proved theorems on long-range order for arbitrary dimensions; Brandt and Mielsch ${ }^{4}$ obtained an exact solution in infinite dimensions; and Jedrzejewski et. al. ${ }^{5}$ performed numerical studies in two dimensions. In this contribution we present additional rigorous results and restricted phase diagrams for the one-dimensional spinless Falicov-Kimball model at $T=0$.

The Hamiltonian for the one-dimensional spinless Falicov-Kimball model defined on a lattice of $N$ sites with periodic boundary conditions (PBC) is

$$
H=-\imath \sum_{j=1}^{N}\left(c_{j}^{\dagger} c_{j+1}+c_{j+1}^{\dagger} c_{j}\right)+U \sum_{j=1}^{N} c_{j}^{\dagger} c_{j} W_{j}
$$

where $c_{j}^{\dagger}\left[c_{j}\right]$ are fermionic creation [annihilation] operators for a spinless ${ }^{6}$ electron at site $j, W_{j}$ is a classical variable that is 1 [0] if an ion occupies [does not occupy] the $j$ th site of the lattice, $t$ is the hopping integral between nearest neighbors, and $U$ is the ion-electron on-site interaction. The first term in (2.1) is the kinetic energy of the itinerant electrons and the second term is the interaction between electrons and ions. 
The total electron number $N_{e}=\sum_{j=1}^{N} c_{j}^{\dagger} c_{j}$ and the total ion number $N_{i}=\sum_{j=1}^{N} W_{j}$ are both conserved quantities.

The Hamiltonian (2.1) for the Falicov-Kimball model bas various physical interpretations. It was originally introduced to examine the mutual interaction of conduction electrons (our electrons) with localized $d$ - or $f$-electrons (our ions) in transition-metal or rare-earth compounds. ${ }^{1}$ It has recently been proposed as a model for crystalline formation ${ }^{3}-$ if the icn configuration $\left\{W_{j}\right\}$ of the ground-state is periodic, then this model provides a mechanism for electron-induced crystalline order. It also describes a one-dimensional binary alloy problem with the following map: occupied site $\rightarrow$ ion of type A; empty site $\rightarrow$ ion of type B; and $U \rightarrow U_{A}-U_{B}$ the difference in electron-ion site energy between ions of type $A$ and type $B$. Note that the Hamiltonian (2.1) is also identical to the one-dimensional tight-binding Schrbdinger equation with an on-site potential that can assume two different values ( 0 and $U$ ). The tight-binding Schrddinger equation has been studied for random $\left\{W_{j}\right\}$ by mathematicians and physicists ${ }^{7}$ and has been investigated recently for aperiodic deterministic sequences. ${ }^{8}$

Since the electrons do not interact among themselves, the energy levels of (2.1) are determined by the eigenvalues of $H$ and the ground-state energy of a particular ion configuration $I \equiv\left\{W_{j}\right\}$ is found by filling in the lowest $N_{e}$ one-electron levels. We let $E^{\Gamma}\left(X, N_{e}\right)$ denote the ground-state energy for $N_{e}$ electrons in the ion configuration $\Gamma$ with $X \equiv U / t$ (the hopping integral $t$ determines the energy scale; all energies are measured in units of $t$ ). Many-body effects enter into the problem by considering the ground state for $N_{i}$ ions

$$
E\left(X, N_{e}, N_{i}\right)=\min \left[E^{\Gamma}\left(X, N_{e}\right) \mid N_{i}=\sum_{j=1}^{N} W_{j}\right\} \quad,
$$

determined by comparing the $\left[N ! / N_{i} !\left(N-N_{i}\right) !\right]$ ion configurations with fixed ion nurnber. The minimization procedure in (2.2) determines the equivalence class of tie 
ground-state ion configuration as a function of the interaction strength, the number of electrons and the number of ions.

The Hamiltonian exhibits two kinds of particle-hole symmetries ${ }^{3}-$ an ion occupied-empty site symmetry and an electron-hole symmetry. In the frst case, the conjugate ion configuration $\Gamma^{\prime \prime}$ is defined by interchanging occupied and unoccupied sites in the configuration $\Gamma$ (this corresponds to $w_{j}^{*}=1-W_{j}$ ). The ground states for these two configurations are related

$$
E^{\Gamma}\left(X, N_{e}\right)=E^{\Gamma}\left(-X, N_{e}\right)+X N_{e} \quad,
$$

for all $X$ and $N_{e}$. In the second case, the unitary transformation $c_{j} \rightarrow(-1)^{j} c_{j}$ and $c_{j}^{\dagger} \rightarrow(-1)^{j} c_{j}^{\dagger}$ is used to relate electron eigenvalues with interaction $X$ to corresponding hole eigenvalues with interaction $(-X)$ yielding the result

$$
E^{\Gamma}\left(X, N_{e}\right)=E^{\Gamma}\left(-X, N-N_{e}\right)+X N_{i}
$$

These two symmetries are employed to reduce the necessary parameter space in the calculation of the $T=0$ (ground-state) phase diagrams.

In the thermodynamic limit the number of lattice sites becomes infinite $(N \rightarrow \infty)$ but the electron $p_{e} \equiv N_{e} / N$ and the ion $p_{i} \equiv N_{i} / N$ concentrations remain finite. The ground-state energy per lattice site is determined from $n^{\Gamma}(E)$ the density of states (DOS)

$$
E^{\Gamma}\left(X, \rho_{e}\right)=\int_{-\infty}^{E_{F}} n^{\Gamma}(E) E d E
$$

where $E_{F}$ is the Fermi level and

$$
\rho_{e}=\int_{-\infty}^{E_{F}} n^{\Gamma}(E) d E
$$

for each ion configuration $\Gamma$. The DOS is calculared from Green's function by

$$
n(E)=-\frac{1}{\pi} \operatorname{Im} \lim _{\varepsilon \rightarrow 0} G(E+i \varepsilon)
$$




$$
G(E)=\frac{1}{N} \sum_{j=1}^{N} G_{j}(E)
$$

where the local Green's function is defined by the marix element $G_{j}(E) \equiv\langle j|1 /(E-H)| j\rangle$. A renormalized perturbation expansion is used to determine the local Green's function exactly. The result ${ }^{9}$

$$
G_{j}(E)=\frac{1}{E-X W_{j}-\Delta_{j}^{+}(E)-\Delta_{j}^{-}(E)}
$$

is expressed in terms of continued fractions

$$
\Delta_{j}^{ \pm}(E)=\frac{1}{E-X W_{j \pm 1}-\frac{1}{E-X W_{j \pm 2}-\frac{1}{E-X W_{j \pm 3}-\cdots}}}
$$

where the local self-energy is $\Delta_{j}(E)=\Delta_{j}^{+}(E)+\Delta_{j}^{-}(E)$.

The continued fractions in (2.9) are evaluated straightforwardly for any periodic configuration $\Gamma$ since the variables $W_{j}$ are then periodic and the fraction may be made finite. For example, the period-two case is analyzed by

$$
\Delta_{0}^{ \pm}(E)=\frac{1}{E-X W_{1}-\frac{1}{E-X W_{0}-\Delta_{0}^{ \pm}(E)}}
$$

which yields

$$
\begin{aligned}
\Delta_{0}^{ \pm}(E) & =\frac{1}{2}\left(E-X W_{0}\right. \\
& \left. \pm\left[\left(E-X W_{0}\right)^{2}\left(E-X W_{1}\right)^{2}-4\left(E-X W_{0}\right)\left(E-X W_{1}\right)\right]^{1 / 2} /\left(E-X W_{1}\right)\right]
\end{aligned}
$$

and, for the DOS

$$
n(E)=\frac{1}{2 \pi} \operatorname{Re} \frac{\left|E-X W_{0}\right|+\left|E-X W_{1}\right|}{\sqrt{-\left(E-X W_{0}\right)\left(E-X W_{1}\right)\left[E^{2}-X\left(W_{0}+W_{1}\right) E+X^{2} W_{0} W_{1}-4\right]}}
$$


In addition to the one-phase periodic configurations, one physically relevant twophase configuration (called the segregated phase) is considered. The segregated phase is an incoherent mixture of the empty and full lattices with weights $\left(1-p_{i}\right)$ and $p_{i}$ respectively. The segregated phase has the physical interpretation of the case where the ions clump together and do not form a periodic arrangement (crystallization model) or of the case where the ions of type $A$ and the ions of type $B$ are immiscible and separate (alloy model). The DOS is trivial for the segregated phase since it is a weighted linear combination

$$
n^{\operatorname{seg}}(E)=\left(1-p_{i}\right) n^{e m p t y}(E)+p_{i} n^{\text {full }}(E)
$$

of the DOS for the empty and full lattices.

The segregated phase is also important since it is expected to be the ground state in the limit $|X| \rightarrow \infty$. In this limit the potential barrier is so large that the electrons are trapped between ion occupied-empty site boundaries. The dominant contribution to the ground-state energy is the kinetic energy of the electrons which is minimized by making the box as large as possible. This favors the segregated phase to be the ground state. However, at the point where the electrons completely fill the box $\left(p_{e}=1-p_{i}\right.$ for $X \rightarrow+\infty$ and $\rho_{e}=p_{i}$ for $\left.X \rightarrow-\infty\right)$ the Pauli exclusion principle requires the additional electrons to be placed above a large potential barrier. At this point a periodic arangement of the ions may actually lower the ground-state energy. These physical ideas are summarized in what is called the segregation principle: In the limit $|X| \rightarrow \infty$ the segregated phase is the ground state for all values of the electron concentration except the specific values $p_{e}=1-p_{i}$ for $X \rightarrow+\infty$, and $p_{e}=p_{i}$ for $X \rightarrow-\infty$. The segregation principle is found to be true in all calculated cases and is expected to hold for all values of $\rho_{e}$ and $p_{i}$.

In the following section perturbation theory is used to analyze the structure of the ground-state phase diagram near $X=0$. In Sections II.3 and II.4 the cases with ionic densities of $p_{i}=1 / 2$ and $\rho_{i}=1 / 3$ respectively are examined, and complete phase 
diagrams for the segregated phase and all ionic configurations with periods less than 10 compatible with those $\rho_{i}$ are given. Conclusions are presented in the final section.

\subsection{Perturbative Analysis}

In the limit $X \ll 1$ a perturbative analysis of the Hamiltonian (2.1) can be performed to determine the structure of the phase diagrams for small interaction strength. Only periodic structures are considered to avoid the technical difficulties associated with aperiodic configurations. Suppose the configuration $\Gamma(r)$ has period $r$; that is, $W_{j+r}=W_{j}$ for all $j$. The Fourier coefficient $W(2 \pi n / r)$ is defined

$$
W(2 \pi n / r) \equiv \frac{1}{N} \sum_{j=1}^{N} e^{-i \frac{2 \pi n j}{r}} W_{j}=\frac{1}{r} \sum_{j=1}^{r} e^{-i \frac{2 \pi n j}{r}} W_{j}
$$

for $n=0,1, \cdots, r-1$. Straightforward Rayleigh-Schrbdinger perturbation theory through second-order, with the second term in (2.1) as the perturbation, yields the expression

$$
\begin{aligned}
E^{\Gamma(r)}\left(X, \rho_{e}\right) & =-\frac{2}{\pi} \sin \pi \rho_{e}+X \rho_{e} \rho_{i} \\
& +\frac{X^{2}}{8 \pi} \sum_{n=1}^{r-1} \frac{|W(2 \pi n / r)|^{2}}{\sin \pi n / r} \log \left|\frac{\sin \pi n / r-\sin \pi \rho_{e}}{\mid \sin \pi n / r+\sin \pi \rho_{e}}\right|+O\left(X^{3}\right)
\end{aligned}
$$

for the ground-state energy of configuration $\Gamma(r)$. The minimization procedure (2.2) outlined above considers configurations with the same ion concentration $\left(\rho_{i}\right)$ at fixed electron concentration $\left(\rho_{e}\right)$ and interaction strength $(X)$, so that the ground-state energy is degenerate up to first order. The second-order term has a logarithmic singularity at $p_{e}=n / r$ with relative strength $|W(2 \pi n / r)|^{2}$. The singularity indicates that perturbation theory fails at these critical electron concentrations; by comparing the strength of the singularity for different configurations, the ground state can be determined in the region near $\rho_{e}=n / r$ (and by continuity at $\rho_{e}=n / r$ ). 
In fact, if the minimization in (2.2) is restricted to be only over periodic configurations, then for rational concentrations the ground-state configuration has the lowest allowed periodicity (this is expected from a Fermi-surface nesting argument: the state with the largest gap at the Fermi level is the ground state). More rigorously the following theorem is proven:

Theorem 2.1. Given rational electron and ion concentrations

$$
\rho_{e}=\frac{p_{e}}{q_{e}}, \quad \rho_{i}=\frac{p_{i}}{q_{i}}
$$

with $p_{c}$ relatively prime to $q_{e}$ and $p_{i}$ relatively prime to $q_{i}$, then the periodic configuration with the lowest energy has period $Q=\operatorname{LCM}\left(q_{x}, q_{i}\right)_{2}$ where LCM stands for least common multiple. The proof is given in the Appendix and includes an expression for the ion configuration $\Gamma(Q)$ corresponding to the lowest-energy state.

These lowest-energy configurations satisfy certain structural properties. Let $l$ denote the length of the largest connected island of occupied sites in the configuration

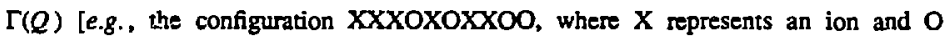
represents an empty site, corresponds to a given $\Gamma(10)$ and has $l=3$ ], then a configuration in which only islands of length $l$ and $(l-1)$ appear is defined to hrve the uniform ion distribution property. For example, XXOXXOOO has the uniform ion distribution property but XXXOOXOO does not. The uniform empty-site distribution property is analogously defined. This characterization of the ground-state configuration in the limit $X \rightarrow 0$ is summarized in the following theorem:

Theorem 2.2. In the limit $X \rightarrow 0$ any periodic lüwest-energy configuration with $p_{i} \leq 1 / 2$ has the uniform ion distribution property and any periodic lowest-energy configuration with $p_{i} \geq 1,2$ has the uniform empty-site distribution property. The proof is given in the Appendix.

The ground-state energy of the segregated phase also has a perturbative expansion about $X=0$. A straightforward analysis using the DOS in equation (2.13) yields 


$$
E^{\text {seg }}\left(X, \rho_{e}\right)=-\frac{2}{\pi} \sin \pi \rho_{e}+X \rho_{e} \rho_{i}-\frac{X^{2}}{4 \pi} \frac{\rho_{i}\left(1-\rho_{i}\right)}{\sin \pi \rho_{e}}+O\left(X^{3}\right)
$$

for the ground-state energy of the segregated phase which is valid in the two-phase, band overlap region $\delta(X)<\rho_{e}<1-\delta(X)$ where

$$
\delta(X)=\frac{\sqrt{|X|}}{\pi}\left[\theta(-X) p_{i}+\theta(X)\left(1-p_{i}\right)\right],
$$

and $\theta(X)$ is the unit step function. This expansion has a singularity in the limit $X \rightarrow 0$ and $p_{e} \rightarrow 0$ which indicates the segregated phase should be the ground state for low electron concentrations.

The solution for the ground-state configuration of the one-dimensional FalicovKimball model is conveniently summarized in a coherent phase diagram. The ion concentration is fixed at $p_{i}=p_{i} / q_{i}$ and the ground-state configuration is plotted as a function of the electron concentration and the interaction strength. The segregated phase is chosen as the zero of the energy scale because of its physical relevance. We limit ourselves to the case $p_{i} \leq 1 / 2$ and $p_{e} \leq 1 / 2$ since the other cases can be obtained by application of the symmetries (2.3) and (2.4). The two theorems above indicate that in the limit $X \rightarrow 0$ the coherent phase diagram has a discontinuous, fractal structure, with a different periodic ground-state configuration at each rational $\rho_{e}$. These configurations all satisfy the relevant uniform-distribution property and appear to be a regular transition from the segregated phase at $\rho_{e} \rightarrow 0$ to a period $q_{i}\left[2 q_{i}\right]$ state at $p_{e}=1 / 2$ if ${ }_{4}$. is even [odd]. The inclusion of aperiodic configurations is not expected to change this general picture. Recent analysis ${ }^{8}$ indicates that sorrs aperiodic configurations have gaps at rational numbers (where the periodic configurations are expected to be lower in energy) aid at irrational numbers (where the aperiodic configurations may be lower in energy). Therefore, we conjecture that in the limit $X \rightarrow 0$ the ground-state configuration changes, point by point, at every value of $\rho_{e}$ and the coherent phase diagram has a regular (discontinuous) transition pattern from the 
segregated phase at $p_{e} \rightarrow 0$ to a periodic phase at $p_{e}=1 / 2$. We also conjecture that the relevant uniform-distribution property holds for each of the ground-state configurations.

\section{II.3 The Case $\rho_{1}=1 / 2$}

In this section the half-full ion case $\left(\rho_{i}=1 / 2\right)$ is examined in detail and the resuits are presented in the form of phase diagrams. We restrict ourselves to the case $p_{e} \leq 1 / 2$ by using the electron-bole symmery (2.4); the phase diagram for the region $\rho_{e} \geq 1 / 2$ is determined by rotating the region $\rho_{e} \leq 1 / 2$ by $180^{\circ}$ about the point $X=0, \rho_{\varepsilon}=1 / 2$. We further restrict ourselves to the case $X \geq 0$ by using the ion occupied-empty site symmetry (2.3); the phase diagram for the region $X \leq 0$ is determined by reflecting the region $X \geq 0$ in a mirror plane along the $X=0$ axis and applying the conjugation operation to the ion configurations (each configuration $\Gamma$ with $\rho_{i}=1 / 2$ is either self-conjugate $\Gamma^{*}=\Gamma$ or forms a conjugation pair with another $p_{i}=1 / 2$ configuration). We finally restrict ourselves to consider only the segregated phase, all periodic phases with $\rho_{i}=1 / 2$ and periods less than 9 , and any incoherent mixture of these phases. These periodic phases are summarized in Table 2.1. The ground state energies are calculated exactly using the Green's function technique outlined in Section II.1.

The coherent phase diagrams are determined by comparing the energy of each periodic phase with the energy of the segregated phase and plotting the lowest-energy state as a function of the electron concentration $\rho_{\varepsilon}$ and the interaction strength $X$. The results are presented in Figures 2.1-2.4 and exhibit the extremely rich structure of the solutions of the model. These resulis are summarized with some observations:

(A) The periodic ground-state theorem and both uniform-distribution properties hold in the region $|X|<1$. 
(B) The alternating phase $X O$ is the ground state at $p_{e}=1 / 2$ for all values of $X$ as stated by previous investigations. ${ }^{23}$

(C) The phase diagrams tend to simplify as the interaction strength increases indicating that many-body effects stabilize the system (this is a consequence of the segregation principle).

(D) There is a trend for phases that disappear from the phase diagram as $X$ increases to reappear as phase-islands at even larger values of $X$ (e.g., the XXXOOO phase in Figs. 2.3 and 2.4 and the XXXX0000 phase in Fig. 2.4).

(E) Phase-islands of configurations not present at $X=0$ may form at larger values of $X$ (e.g., the XXOXXOOO phase in Fig. 2.4).

(F) The uniform-distribution properties may not hold at finite values of $X$ (the XXOXXOOO phase in Fig. 2.4 does not satisfy the uniform empty-site distribution property and its conjugate does not satisfy the uniform ion distribution property).

(G) Some configurations are not the ground state for any value of $X$ or $P_{e}$ (e.g., the configurations XXXOXOOO and XXOXOXOO do not appear in Fig. 2.4).

The incoherent phase diagrams are determined by choosing the minimal onergy state, allowing for incoherent mixing ${ }^{10}$ of the $p_{i}=1 / 2$ periodic phases with themselves and with the segregated phase (which is already an incoherent mixture of the $p_{i}=0$ and $p_{i}=1$ phases). This is accomplished by constructing the convex hull of the ground-state energy curves for fixed $X$ and assigning an incoherent phase mixture to each region where the convex hull is lower than the energy curves. The results are presented in Figures 2.5-2.8 where solid lines and shaded regions correspond to single phases, dashed lines correspond to two-phase mixnures and dotted lines correspond to more than two-phase mixnures (the points where vertical dotted lines pass through horizontal solid lines are the points of phase transitions). The numbers above the single phase lines identify the ground state according to the numbers in Table 2.1. The unshaded region below the dashed line is the region where the segregated phase is the 
ground state. The unshaded regions between a solid [or dashed] line and a solid line are the regions where an incoherent mixture of the two [or three] phases is the ground state. The following observations may be made:

(H) The incoherent phase diagrams are simpler than their single-phase counterparts. The regions enclosing finite areas of single phases are drastically reduced.

(I) The behavior in the limit $|X| \rightarrow 0$ appears to be the same as that predicted by perturbation theory for the coherent diagrams.

(J) The secondary phase-islands that sometimes form at $|X|>0$ either become single phase-lines $(\mathrm{XXXOOO}$ in Figs. 2.7 and 2.8) or vanish altogether $(\mathrm{XXXX0000}$ in Fig. 2.8).

These incoherent phase diagrams are important to study for two reasons: first, they determine the ground state of a real system since any physical system organizes itself in an incoherent mixture of phases to minimize energy (if possible); second, they produce a better approximation to the complete phase diagram of the FalicovKimball model. This is because any incoherent mixture of phases can be reinterpreted as an aperiodic configuration in a coherent phase diagram. By using this reinterpretation the perturbation theory results of Section II.2 are strengthened to conjecture that the ground-state configuration is the segregated phase for a finite region $\left[0 \leq p_{\varepsilon}<\rho_{\varepsilon}^{\max }(X)\right]$; above this region the ground-state configuration changes point by point with $p_{e}$, and has a regular (discontinuous) transition from the segregated phase to a periodic phase at $p_{e}=1 / 2$. Furthermore, for the case $p_{i}=1 / 2$ the inequality $P_{e}^{\max }(X)<1 / 2$ must hold since the altemating state $X O$ is the ground state ${ }^{2.3}$ at $\rho_{e}=1 / 2$ for all $X$. 


\section{II.4 The Case $p_{I}=1 / 3$}

The one-third-full ion case $\left(p_{i}=1 / 3\right)$ is examined as a representative of the general case because it does not bave any extra symmetries. The electron-hole symmetry (2.4) allows us to consider only the case $p_{e} \leq 1 / 2$, but we must consider all values of $X$ since the ion occupied-empty site symmetry (2.3) produces the phase diagrams for the $\rho_{i}=2 / 3$ case. We consider only the segregated phase, the period-three, -six, and -nine phases with $\rho_{i}=1 / 3$, and any incoherent mixture of these phases. ${ }^{10}$ The precise ion configurations considered are summarized in Table $\mathbf{2 . 2}$.

The results for the coherent phase diagrams are presented in Figures 2.9-2.11 and they exhibit a marked asymmetry with respect to the $X=0$ plane. The following observations may be made:

(K) There is no evidence in support of or against the uniform ion distribution property since this property can only be observed for periods 12 and larger, which are not studied here.

(L) The periodic ground-state theorem holds for $|X| \ll 1$ but the many-body effects rapidly become more important and change the structure of the phase diagrams.

(M) It appears that the period-three phase XOO is the ground state at $p_{e}=1 / 3$ for all values of $X$ less than zero.

(N) The segregation principle holds; In the limit $|X| \rightarrow \infty$ the segregated phase is the ground state for all values of $p_{e}$ except for a region about $p_{e}=1 / 3$ and $X \rightarrow-\infty$.

(O) There is still a trend for phases present at $X=0$ to appear as phase-islands at larger values of $|X|$ (e.g., the XXO000 phase in Figs. 2.10 and 2.11; the XOXOOO phase in Fig. 2.10; the XXXOOOOOO phase in Fig. 2.11; and the XXOOOXOOO phase in Fig. 2.11). 
(P) All studied configurations are the ground state for some value of the parameters $p_{e}$ and $X$.

The results for the incoherent phase diagrams are summarized in Figures 2.122.14. We present the following observations:

(Q) Observations (H), (I), and (J) of the previous section still hold.

(R) Two phases (XXOOXOOOO and XOXOOXOOO) do not appear in the incoherent phase diagram although they were present in the coherent phase diagram.

The structure for the full Falicov-Kimball model in the general case emerges from these incoherent phase diagrams. If an incoherent mixture of phases is reinterpreted as an aperiodic phase in the coherent phase diagram, then it appears that at each value of $X$ there is a finite region where the segregated phase is the ground state. In the remaining region the ground state changes from point to point with $\rho_{e}$ and has a regular (discontinuous) transition from the segregated phase to a periodic phase (and possibly) back to the segregated phase.

\section{IL.5 Conclusion}

Since its introduction twenty years ago, the Falicov-Kimball model ${ }^{1}$ is one of the simplest models of interacting electron systems. We have studied the one-dimensional spinless version of this model by exact numerical calculation for a restricted number of phases and by perturbation theory for small interaction strength. Our rigorous results include a periodic ground-state theorem and uniform ion ano empty-site distribution properties for rational electron and ion concentrations and small interaction strength. Our numerical calculations indicate that the phase diagram of the complete model is separated into two distinct regions: In the first region the segregated phase is the ground state; and in the second region the phase diagram has a complex structure with the ground state apparently changing point by point at every value of the electron concentration for fixed interaction strength. In this second region the ground state has a 
regular (discontinuous) transition pattern from the segregated phase to a periodic phase and back to the segregated phase.

Two unproven conjectures are presented that characterize further the structure of the phase diagram as illustrated by the numerical work The first is called the segregation principle which states at large interaction strength the segregated phase is the ground state for almost all electron concentrations. The only exceptions are when the electron concentration matches the ion or the empty-site concentration, where a periodic phase is the ground state. The second is the uniform ion or empty-site distribution property which states that the ground state configurations satisfy certain structural characteristics. The properties are true for small interaction strength but appear to be violated for moderate interactions.

One final open question remains: The proper incoherent phase diagram is plotted as a function of both the electron and ion concentrations. Restricted phase diagrams have been evaluated for only five ion concentrations $\left(p_{i}=0,1 / 3,1 / 2,2 / 3,1\right)$ and there is no concrete conjecture for the structure of the incoherent phase diagram. However, this phase diagram is expected to separate into two regions with simple behavior in one region and complex behavior in the other.

\section{II.6 Appendix: Proof of the Periodic Ground-State Theorems}

In this appendix the two tis...ems stated in Section II. 2 are proven. We begin with the periodic ground-state thecrem:

Theorem 2.1. Given rational electron and ion concentrations

$$
\rho_{e}=\frac{p_{e}}{q_{e}}, \quad p_{i}=\frac{p_{i}}{q_{i}} \text {, }
$$

with $p_{e}$ relatively prime to $q_{e}$ and $p_{i}$ relatively prime to $q_{i}$, then the periodic configuration with the lowest energy has period $Q=\operatorname{LCM}\left(q_{e}, q_{i}\right)$.

Proof: The periodic configuration with the lowest energy is the configuration with the 
largest square Fourier coefficient $\left|W\left(2 \pi \rho_{e}\right)\right|^{2}$. The trial configurations that have non-zero Fourier coefficient and proper ion concentration must have a periodicity that is a multiple of $Q$. Consider all periodic configurations with ion concentration $p_{i}$ and with period less than or equal to $r=m Q$. These configurations all lie on a latrice with $\mathrm{PBC}$ and size $N=M Q$ where $M=\operatorname{LCM}(1,2, \cdots, m)$. We show the configuration with the lowest energy in this restricted set has period $Q$ which (since $m$ is arbitrary) proves the theorem.

The proof proceeds by construction of the largest $\left|W\left(2 \pi p_{c}\right)\right|^{2}$. Assume, for simplicity, that $q_{e}=Q$. Define integers $k_{i}$ by the relation

$$
\left(p_{e} k_{i}\right) \bmod Q=i \quad, \quad i=0,1, \cdots, Q-1 .
$$

Then the choice of $W_{j}=1$ for

$$
j=k_{i}+l Q \quad, \quad i=0,1, \cdots, Q p_{i} l q_{i}-1 \quad, \quad l=0,1, \cdots, M-1
$$

gives an ion concentration

$$
\rho_{i}=\frac{1}{N} \sum_{j=0}^{N-1} W_{j}=\frac{p_{i}}{q_{i}}
$$

and maximizes the square Fourier coefficient

$$
\begin{aligned}
\left|W\left(2 \pi p_{e}\right)\right|^{2} & =\frac{1}{N^{2}} \sum_{j, k} W_{j} W_{k} \cos 2 \pi p_{e}(j-k) / Q \\
& =\frac{1}{Q^{2}}\left[Q p_{i}+2 \sum_{j=1}^{Q p_{1} / q_{i}-1}\left(Q p_{i} / q_{i}-j\right) \cos 2 \pi j / Q\right],
\end{aligned}
$$

since the summation in (2.23a) has the maximal allowed number of $(j-k) \bmod Q=0$, $(j-k) \bmod Q=1, \cdots$, and $(j-k) \bmod Q=Q p_{i} / q_{i}-1$. The minimal configuration $\Gamma(Q)$ constructed above has period $Q$ which completes the proof. The proof for the case $q_{e} \neq Q$ is similar and is omitted here. The only complication of this case is that the second-order perturbation theory may nat fully lift the degeneracy of the lowestenergy state. These degenerate states all have period $Q$ however, which is sufficient 
to prove the theorem. ${ }^{11}$

As an example, we consider the case $p_{e}=3 / 8$ and $p_{i}=1 / 2$. This gives $Q=8$ with $k_{0}=0, k_{1}=3, k_{2}=6$, and $k_{3}=1$, so that the configuration XXOXOOXO is the lowest energy periodic state in the limit $X \rightarrow 0$.

We continue with the proof of the uniform-distribution properties.

Theorem 2.2. In the limit $X \rightarrow 0$ any periodic lowest-energy configuration with $\rho_{i} \leq 1 / 2$ has the uniform ion distribution property and any periodic lowest-energy configuration with $p_{i} \geq 1 / 2$ has the uniform empty-site distribution property.

Proof: We restrict ourselves to the case $\rho_{i} \leq 1 / 2$ and $\rho_{e} \leq 1 / 2$ since the other cases immediately follow upon application of the symmetries (2.3) and (2.4). Assume that $q_{e}=Q$ (the proof of the more general case is similar and is omitted). The $Q$ integers $\left\{k_{i}\right\}$ can be represented in terms of the first $p_{e}$ integers by

$$
k_{s p_{\varepsilon}+j}=k_{j}+s \quad j=0,1, \cdots, p_{\varepsilon}-1 \quad s=0,1, \cdots, r-1 \quad \text {, }
$$

and

$$
k_{r p_{t}+j}=k_{j}+r \quad j=0,1, \cdots, t-1 \quad \text {, }
$$

where $Q=r p_{e}+t$ and $t<p_{e}$. Since each integer from 0 to $Q-1$ appears in $\left(k_{i}\right)$ once and only once, the nearest neighbors in the first $p_{c}$ integers $k_{0}, k_{1}, \cdots, k_{p_{0}-1}$ are separated by gaps of length $r$ or $r-1$ (there are $t$ neighbors with separation $r$ and $p_{e}-t$ neighbors with separation $\left.r-1\right)$. As the ions are filled in according to the prescription (2.21) of Theorem 2.1, each configuration will satisfy the uniform ion distribution property until the gap between any two nearest neighbors in the original $p_{e}$ ions is filled in. This occurs when $\rho_{i}>1-p_{e}$ which is not possible by the hypothesis and proves the theorem. 


\section{II.7 References for Chapter II}

1 L.M. Falicov and J.C. Kimball, Phys. Rev. Lett. 22, 997 (1969).

2 U. Brandt and R. Schmidt, Z. Phys. B 63, 45 (1986); Z. Phys. B 67, 43 (1987).

3 T. Kennedy and E.H. Lieb, Physica 138A, 320 (1986); E.H. Lieb, Physica 140A, 240 (1986).

4 U. Brandt and C. Mielsch, Z. Phys. B 75, 365 (1989).

5 J. Jedrzejewski, J. Lach, and R. Lyżwa, Physics Letters A134, 319 (1989); Physica A154, 529 (1989).

6 Spin may be introduced by simply doubling the allowed electron occupancy of each lattice site since the electrons do not interact with themselves in this model.

7 B. Simon, Adv. Appl. Math. 3, 463 (1982); E.N. Economou, C.M. Soukoulis and M.H. Cohen, Phys. Rev. B 37, 4399 (1988).

8 K. Machida and M. Nakano, Phys. Rey. B 34, 5073 (1986); 2. Cheng, R. Savit and R. Martig, Phys. Rev. B 37, 4375 (1988); J.M. Luck, Phys. Rev. B 39, 5834 (1989); D. Wurtz, T. Schneider, A. Politi and M. Zanneti, Phys. Rev. B 39, 7829 (1989)

9 P.W. Anderson, Phys. Rev. 109, 1492 (1958); E.N. Economou and M.H. Cohen, Phys, Rev. B 4, 396 (1971); E.N. Economou, Green's Functions in Quantum Physics, 2nd ed. (Springer Verlag, Heidleberg, 1983).

10 In theory one can mix configurations with any ion concentrations as long as the mixed phase has the proper ion and electron average concentrations. Only the simplest mixtures are formed here.

11 Higher-order perturbation theory may lift any residual degeneracy of this case. 
II.8 Tables for Chapter II

Table 2.1. Periodic configurations for the $p_{i}=1 / 2$ case

\begin{tabular}{rlc}
\hline \hline & Configuration & Conjugate \\
\hline 1 & X0 & 1 \\
2 & XXOO & 2 \\
3 & XXXOOO & 3 \\
4 & XXOXOO & 4 \\
5 & XXXXOOOO & 5 \\
6 & XXXOXOOO & 6 \\
7 & XXXOOXOO & 8 \\
8 & XXOXXOOO & 7 \\
9 & XXOXOXOO & 9 \\
10 & XXOXOOXO & 10 \\
\hline \hline
\end{tabular}


Table 2.2. Periodic configurations for the $p_{i}=1 / 3$ case

\begin{tabular}{|c|c|}
\hline & Configuration \\
\hline 1 & xOO \\
\hline 2 & Xxo000 \\
\hline 3 & xoxooo \\
\hline 4 & XxxO000000 \\
\hline 5 & XXox00000 \\
\hline 6 & XX00X0000 \\
\hline 7 & xxo00x000 \\
\hline 8 & XOXOX0000 \\
\hline 9 & XOXOOXOOO \\
\hline
\end{tabular}




\section{II.9 Figures for Chapter II}

Figure 2.1. Calculated coherent phase diagram for the segregated and period-two phases with $\rho_{i}=1 / 2$. See Table 2.1 for the key to the legend.

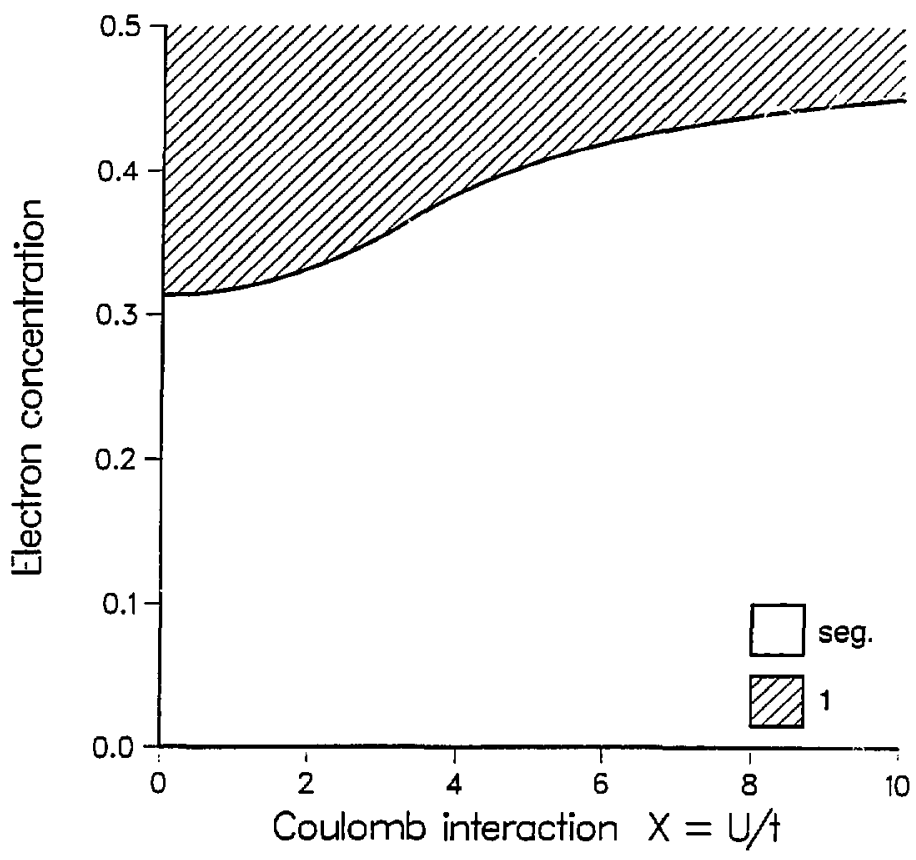


Figure 2.2. Calculated coherent phase diagram for the segregated, period-two and -four phases with $\rho_{i}=1 / 2$. See Table 2.1 for the key to the legend.

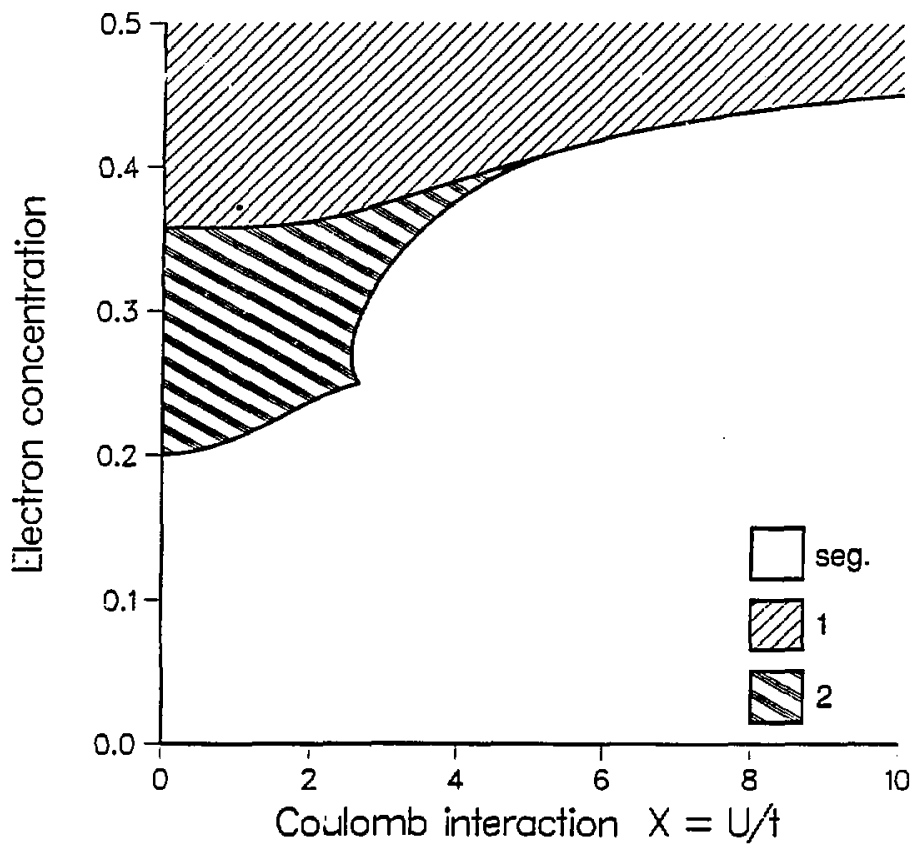


Figure 2.3. Calculated coherent phase diagram for the segregated, period-two, -four and - six phases with $p_{i}=1 / 2$. See Table 2.1 for the key to the legend.

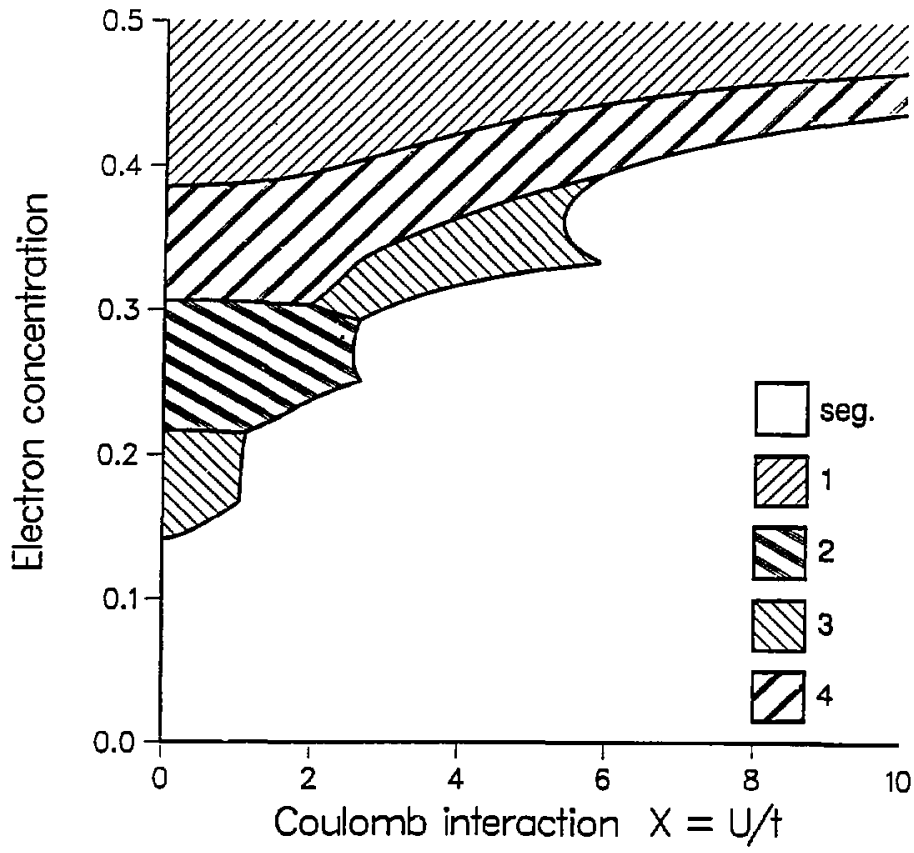


Figure 2.4. Calculated coherent phase diagram for the segregated, period-two, -four, -six and -eight phases with $p_{i}=1 / 2$. See Table 2.1 for the key to the legend.

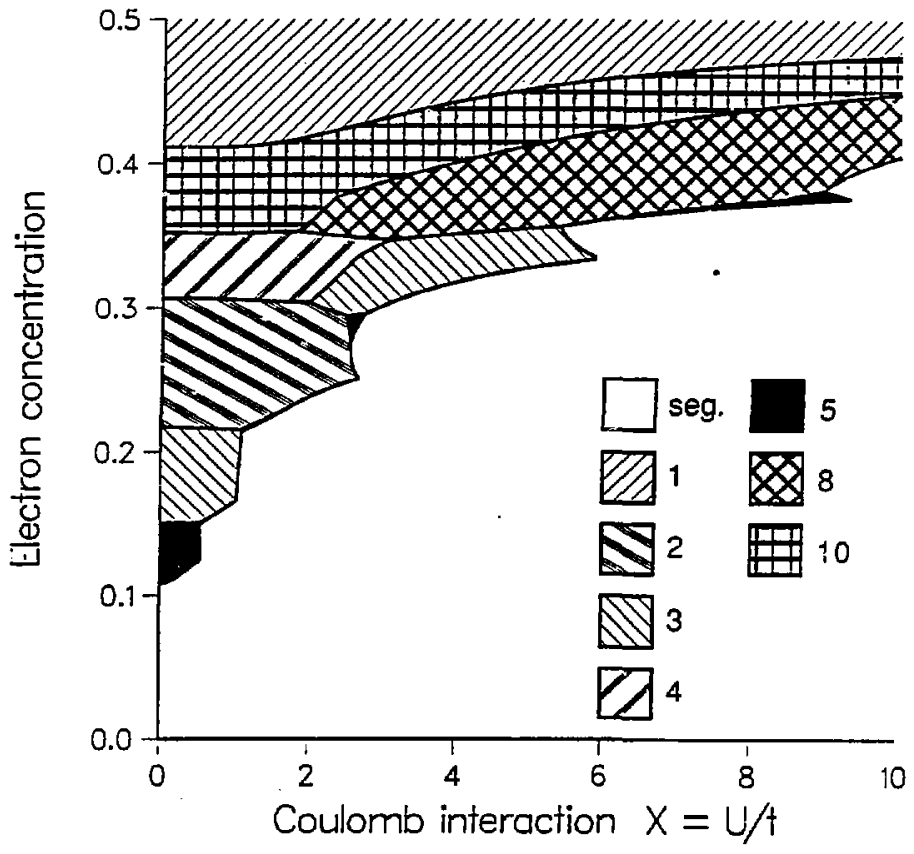


Figure 2.5. Calculated incoherent phase diagram for the segregated and period-two phases with $p_{i}=1 / 2$. See Table 2.1 for the key to the legend.

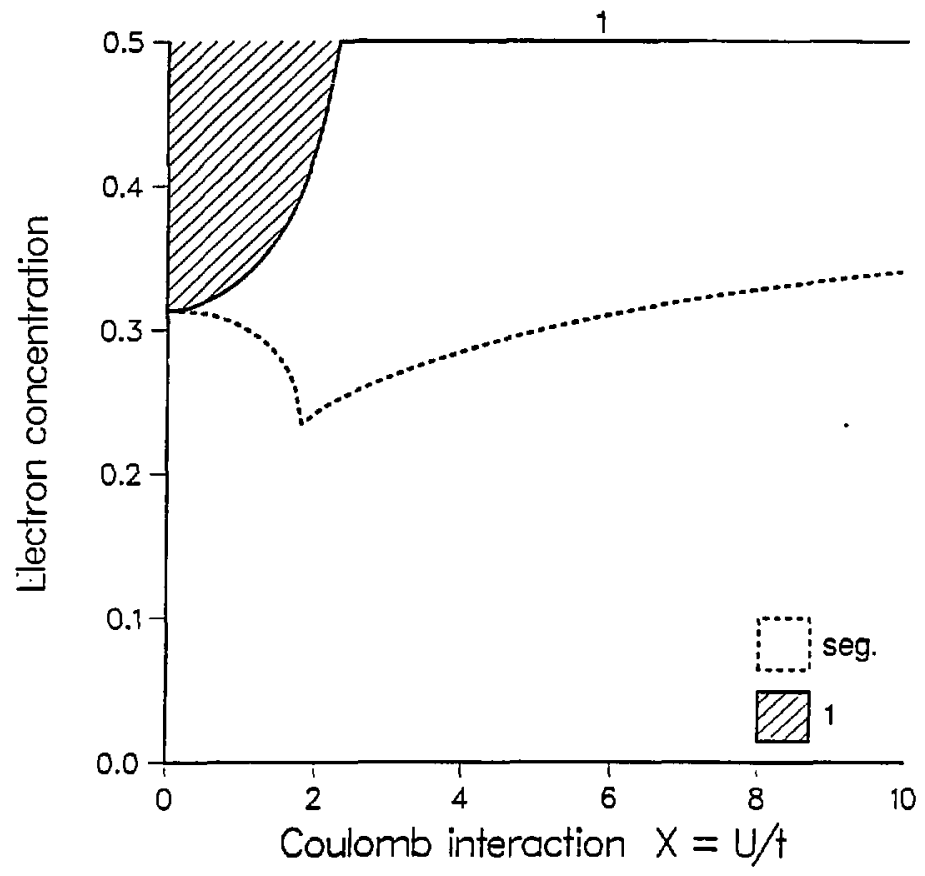


Figure 2.6. Calculated incoherent phase diagram for the segregated, period-two and -four phases with $\rho_{i}=1 / 2$. See Table 2.1 for the key to the legend.

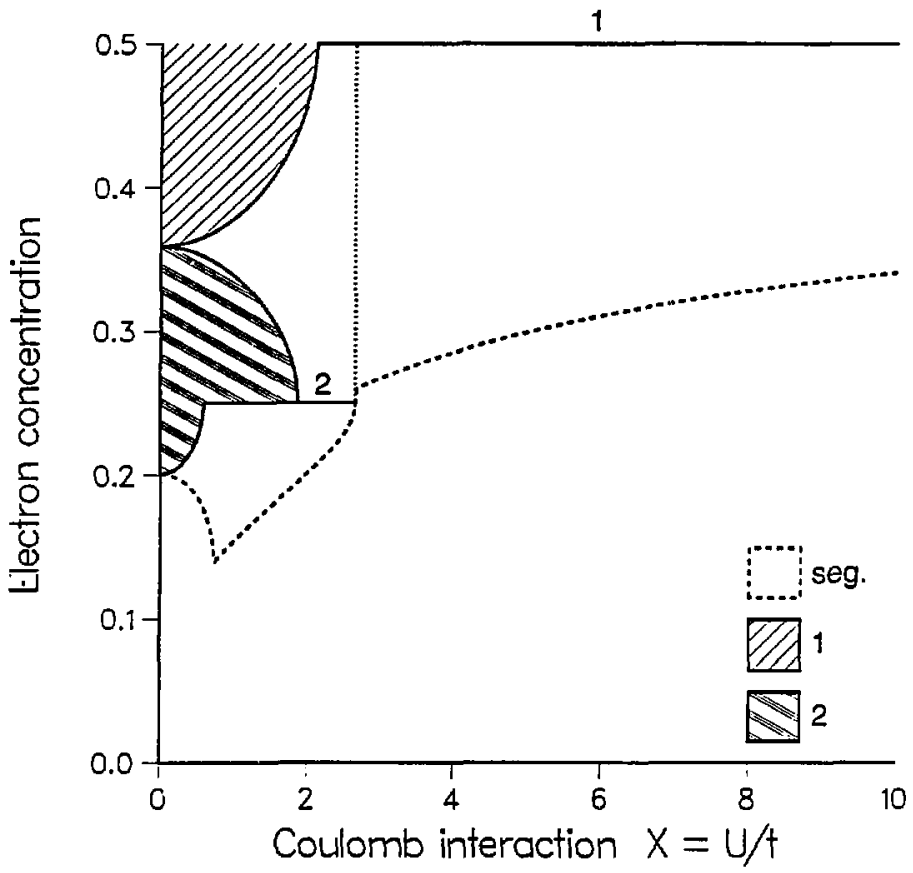


Figure 2.7. Calculated incoherent phase diagram for the segregated, period-two, -four and -six phases with $p_{i}=1 / 2$. See Table 2.1 for the key to the legend.

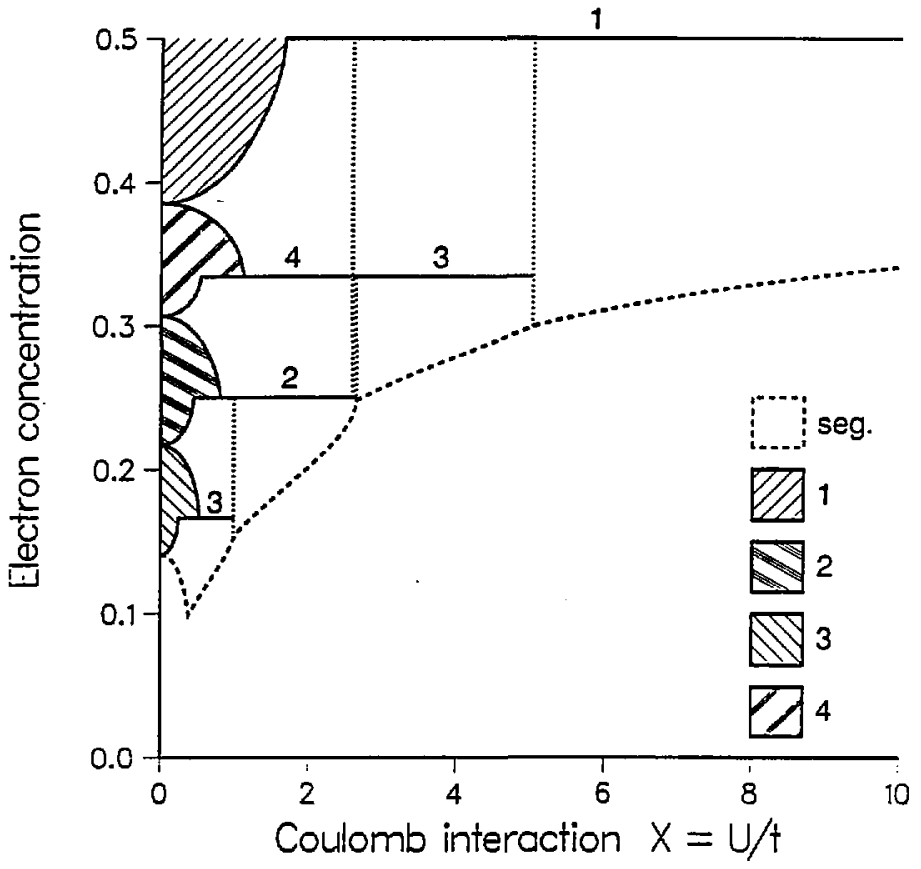


Figure 2.8. Calculated incoherent phase diagram for the segregated, period-rwo, -four, -six and -eight phases with $p_{i}=1 / 2$. See Table 2.1 for the key to the legend.

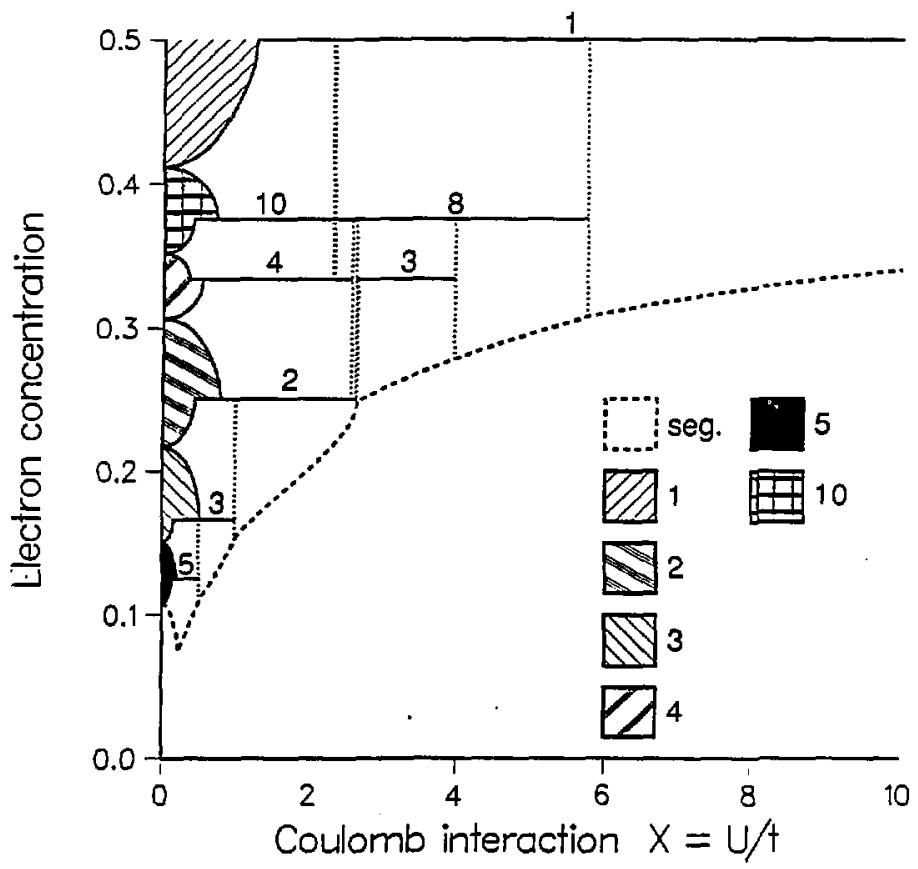


Figure 2.9. Calculated coherent phase diagram for the segregated and period-three phases with $p_{i}=1 / 3$. See Table 2.2 for the key to the legend.

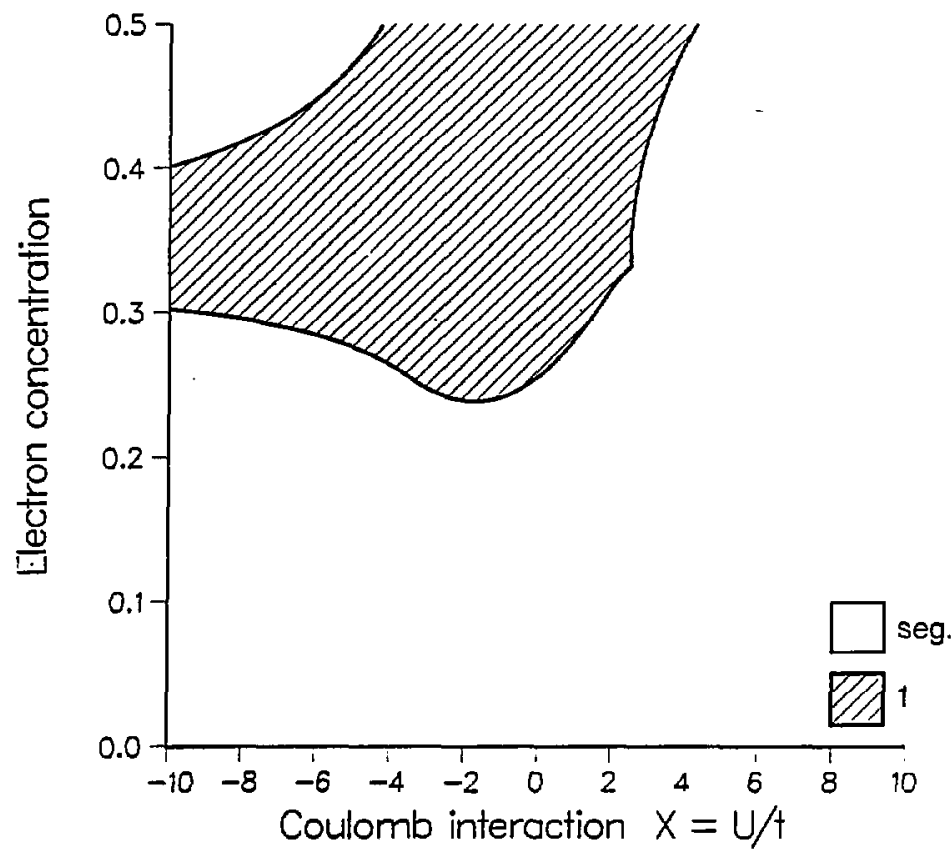


Figure 2.10. Calculated coherent phase diagram for the segregated, period-three and -six phases with $p_{i}=1 / 3$. See Table 2.2 for the key to the legend.

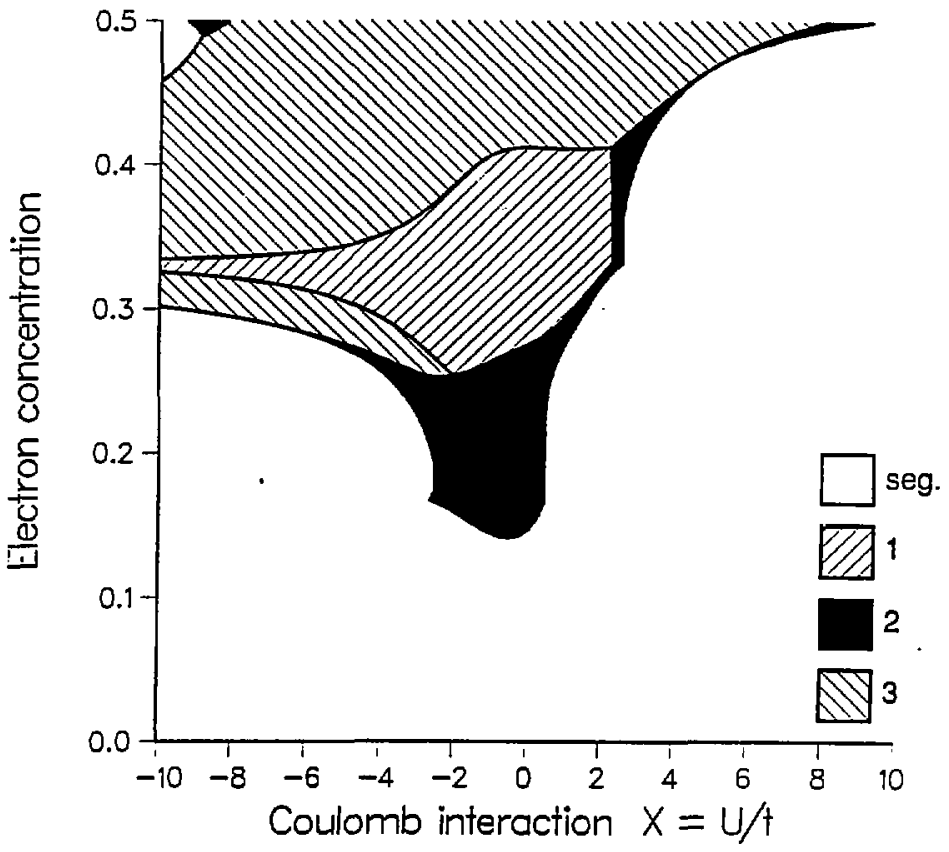


Figure 2.11. Calculated coherent phase diagram for the segregated, period-three, -six and -nine phases with $\rho_{i}=1 / 3$. See Table 2.2 for the key to the legend.

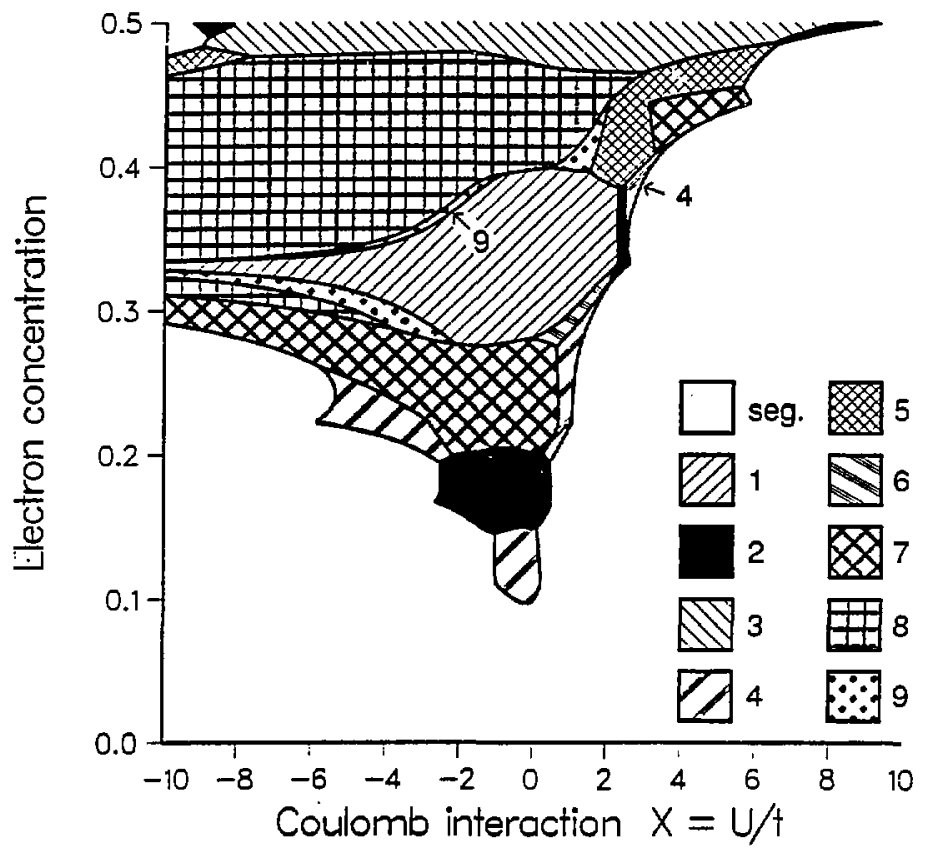


Figure 2.12. Calculated incoherent phase diagram for the segregated and period-three phases with $p_{i}=1 / 3$. See Table 2.2 for the key to the legend.

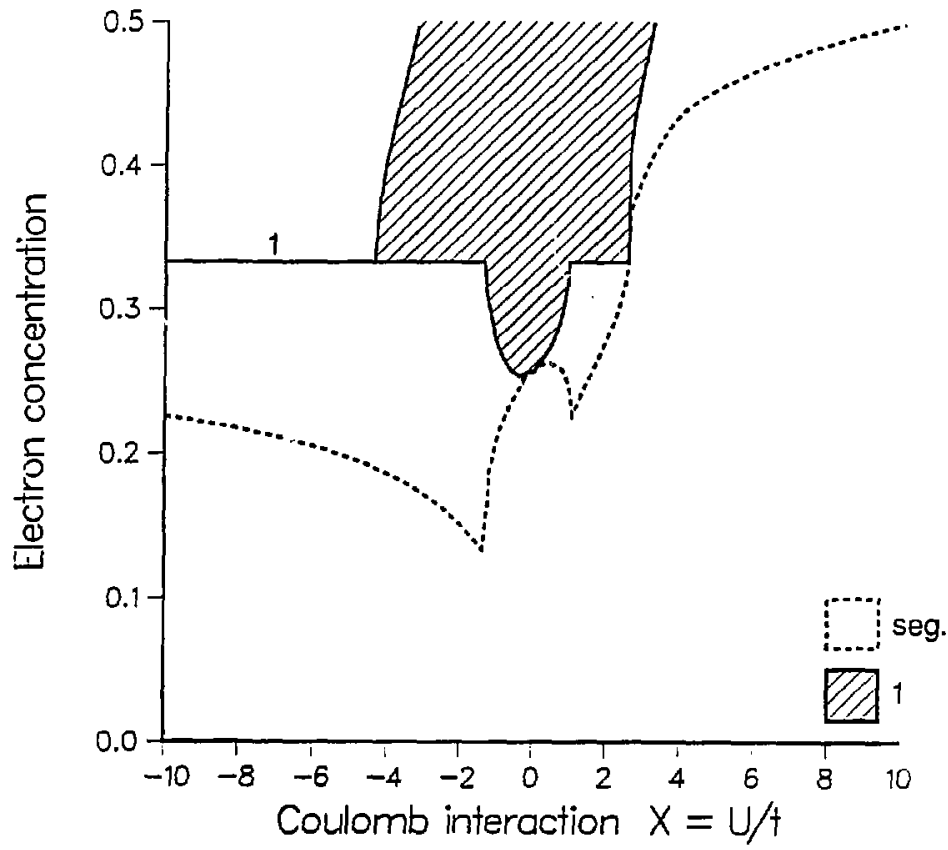


Figure 2.13. Calculated incoherent phase diagram for the segregated, period-three and -six phases with $\rho_{i}=1 / 3$. See $\mathrm{Ta}^{2} \cdot 1 \mathrm{e}, 2$ for the key to the legend.

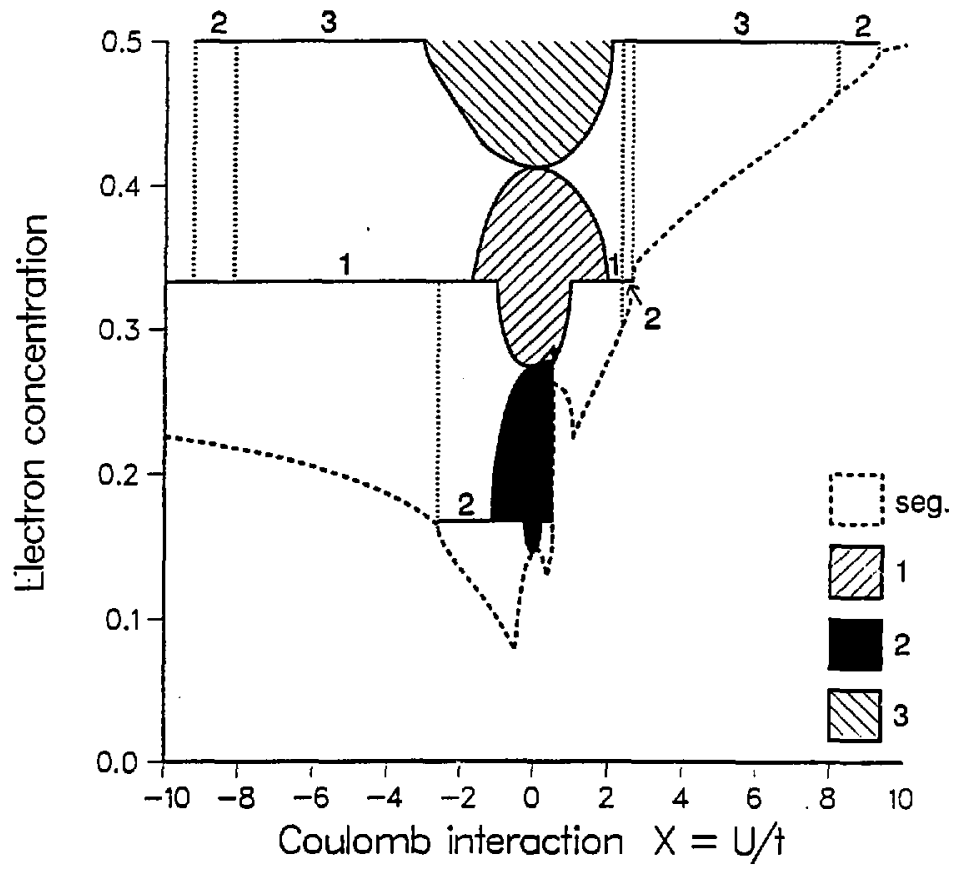


Figure 2.14. Calculated incoherent phase diagram for the segregated, period-three, -six and -nine phases with $p_{i}=1 / 3$. See Table 2.2 for the key to the legend.

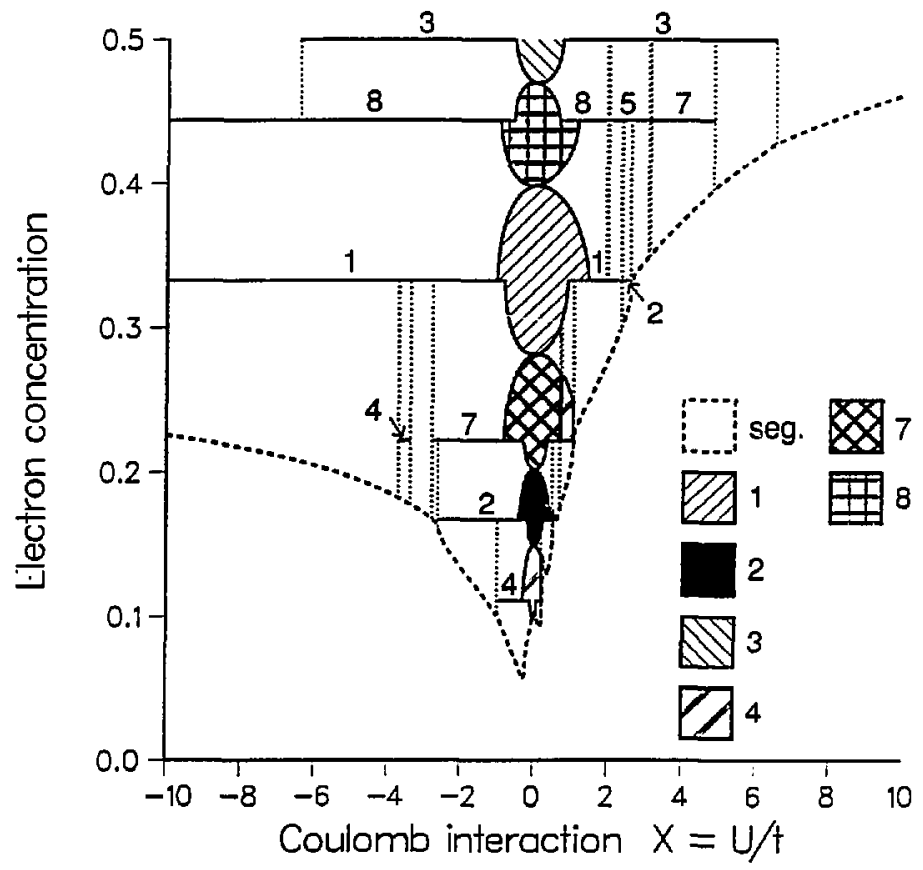




\section{Chapter III: Exact Solution of the $\mathbf{t}-\mathbf{t}^{\prime}-\mathbf{J}$ Model on Eight-Site Cubic Systems}

\section{III.1 Introduction}

Strong electron correlation is responsible for long-range-order magnetic materials, ${ }^{1}$ heavy fermion (HF) behavior, ${ }^{2,3}$ and high-temperature superconductivity. ${ }^{4,5}$ The $t-r^{\prime}-J$ model is the simplest model of an interacting electronic system that mimics the strong correlation effects present in these materials. In ferromagnetic and HF systems this model describes the mutual interaction and effective electron transfer of the narrow $d$ - and $f$-band electrons while in the high-temperature superconductors it approximates the hole-hole interaction and hole hopping in the $\mathrm{CuO}_{2}$ planes.

The $t-t^{\prime}-J$ model is defined on a lattice with one spherically symmetric orbital per site by the following Hamiltonian:

$$
H=H_{1 N N}+H_{2 N N}+H_{\text {int }} \quad .
$$

where ${ }^{6}$

$$
\begin{gathered}
H_{1 N N}=-t \sum_{\substack{i, j: \sigma \\
\langle i, j>=1 N N}}\left(1-n_{i-\sigma}\right) c_{i \sigma}^{\dagger} c_{j \sigma}\left(1-n_{j-\sigma}\right) \\
H_{2 N N}=-t \sum_{\substack{i, j ; \sigma \\
\langle i, j>=2 N N}}\left(1-n_{i-\sigma}\right) c_{i \sigma}^{\dagger} c_{j \sigma}\left(1-n_{j-\sigma}\right) \\
H_{i N \pi}=J \sum_{\substack{i, j \\
\langle i, j>=1 N N}} S_{i} \cdot S_{j} .
\end{gathered}
$$

In these equations $c_{i \sigma}^{\dagger}\left[c_{i \sigma}\right]$ are creation [destruction] operators for an electron in the orbital at site $i$ with z-component of spin $\sigma, n_{i \sigma}=c_{i \sigma}^{\dagger} c_{i \sigma}$ is the corresponding number operator, and $S_{i}$ is the vector spin of an electron at site $i$. The term, in $H$ include a band "hopping" interaction between conduction states on nearest-neighbor sites (3.2a) and next-nearest-neighbor sites (3.2b) and an antiferromagnetic nearest-neighbor Heisenberg superexchange interaction term (3.2c) with exchange integral $2 J$. The 
hopping terms contain projection operators that prevent doubls occupation of any orbital.

This Hamiltonian has two interpretations: it is an electronic system with indirect exchange interactions and a "super" Pauli principle that forbids electrons of like or unlike spin from occupying the same spatial site; or it is an approximation to the $U \rightarrow \infty$ limit of the single-band Hubbard ${ }^{7}$ model

$$
H_{H u b b}=-t \sum_{\substack{i, j j \sigma \\<i, j>=1 N N}} c_{i \sigma}^{\dagger} c_{j \sigma}-t \sum_{\substack{i, j: \sigma \\<i, j>=2 N N}} c_{i \sigma}^{\dagger} c_{j \sigma}+U \sum_{i} n_{i \downarrow} n_{i \uparrow}
$$

Anderson ${ }^{8}$ first showed the equivalence of the half-filled band Hubbard model at large interaction strength to the Heisenberg model. His proof was based upon second-order perturbation theory: At half-filling and infinite $U$ each lattice site is singly occupied and all spin states are degenerate. When $C^{\prime}$ is made finite, the lowest order correction to the energy comes from virtual processes where an electron hops to its nearest neighbor (if the spins are antiparallel) and then hops back. The energy gain for such a fluctuation is $=r^{2} / U$ since doubly occupied states have energy $=U$. This hopping creates the Heisenberg superexchange interaction term to lowest order in $t / U$. Away from half-filling, the electrons can hep from occupied to empty sites and additional fluctuations that involve three sites (an electron hops to a neighboring occupied site and then hops to a third unoccupied site) are present. Schrieffer and Wolff ${ }^{9}$ found a canonical transformation to the single-occupied sector of a related model that was valid for arbitrary fillings. This technique was applied to the Hubbard model to first order, ${ }^{19}$ and recently to arbitrary order. ${ }^{.1}$ Since the $t-t^{\prime}-J$ Hamiltonian (3.1) only involves the nearest-neighbor superexchange interaction, it approximates the canonically transformed Hubbard Hamiltonian (3.3) in the limit of large $U$ when $J=2 t^{2} U$ and when any termis of order $O\left(t^{2} / U^{2}\right)$ or $O\left(t^{2} / t U\right)$ and any three-site hopping terms in the transformed Hubbard Humiltonian are neglected. This approximarion is exact at half-filling for $t^{\prime}=0$ but becomes increasingly less accurate with hole concentration 
away from half-filling.

A few rigorous results are known about the $t-t^{\prime}-J$ model:

(a) At half-filling it reduces to a Heisenberg model whose ground state ${ }^{12}$ is a nondegenerate singlet on bipartite lattices and possibly ferrimagnetic for other cases. Lieb $^{13}$ recently extended this analysis to the Hubbard model with finite $U$.

(b) The case of one hole in a half-filled band at $J=0(U=\infty)$ is known to be ferromagnetic ${ }^{14}$ (Nagaoka's theorem) when $r \leq 0$ for the simple cubic (sc), bodycentered cubic (bcc), and the square (sq) lattices for all $t$ and for the facecentered cubic $(f c c)$ when $t<0$.

(c) The one-dimensional $t-t^{\prime}-J$ model with free boundary conditions and an even number of electrons has a spin-singlet ground state. ${ }^{15}$

(d) The one-dimensional Hubbard model has been solved exactly with the Bethe ansatz fer arbitrary fillings by $\mathrm{Lieb}$ and $\mathrm{Wu}^{16}$ which yields solutions ${ }^{17,18}$ to the $t-r^{\prime}-J$ model at $t^{\prime}=0$ and $J=0(U=\infty)$.

(e) The Bethe ansatz has also been applied ${ }^{19}$ to the one-dimensional $t-t-J$ model with $r=J$ and $t^{\prime}=0$.

Aside from these theorems little else is known rigorously about the solutions of this many-body problem. The standard approach is to apply variational, perturbative, or mean-field approximations to such interacting models. An alternate method is chosen which is exact, but subject to finite-size effects. It is called the small-cluster approach. 20

The small-cluster approach begins with the periodic crystal approximation: ${ }^{21} \mathrm{~A}$ bulk crystal of $M$ atoms is modeled by a latrice of $M$ sites with periodic boundary conditions (PBC). Bloch's theorem then labels the electron many-body wavefunctions by one of $M k$-vectors of the first Brillouin zone. The standard approach takes the thermodynamic limit $(M \rightarrow \infty)$, which replaces the finite grid in reciprocal space by a 
continuum that spans the Brillouin zone. Electron correlation effects are then treated in an approximate fashion. The small-cluster approach takes the opposite limit: The number of sites is chosen to be a small number $(M=8)$ restricting the sampling in momenrum space to a few high-symmetry points. However, the interacting electronic system is solved exactly taking into account all electron correlation effects. The oneelectron band structure of these two methods is identical when sampled at the common points in reciprocal space. The relationship of the many-body solutions (at equal electron concentration) for the macroscopic crystal and the small cluster is much more complicated due to uncontrolled finite-size effects in the latter. Nevertheless, the small-cluster approach does provide an alternate means of rigorously studying the mariy-body problem and (possibly) extrapolating these results to macroscopic crystals.

The small-cluster approach was proposed independently for the Hubbard model by Hartis and Lange ${ }^{10}$ and Falicov and Harris ${ }^{22}$ with the exact solution of a two-site cluster. Subsequent work concentrated on the ground-state ${ }^{23}$ and thermodynamic ${ }^{24}$ properties of the one-dimensional half-filled band Hubbard model on four- and six-site clusters.

The first truly three-dimensional case to be investigated was the eight-site sc cluster. Ground state properties at infinite ${ }^{25}$ and finite ${ }^{26} U$ and thermodynamic ${ }^{25-27}$ properties have all been studied. The solution of the four-site square $(s q)$ and tetrahedral $(f c c)$ clusters $^{28}$ marked the first time that group theory was used to factorize the Hamiltonian into block-diagonal form by using basis functions of definite spin that transform acconding to irreducible representations of the full space group.

Takahashi ${ }^{29}$ studied the ground-state spin as a function of electron filling in the infinite $U$ limit of the Hubbard model on a variety of clusters (up to twelve sites). Unfortunatel" the use of free boundary conditions (instead of PBC) introduces strong surface effects that complicate extrapolation to the thermodynamic limit. The effect of geometry on the ground state has also been examined ${ }^{30}$ for finite U. 
The $t-r^{\prime}-J$ model was solved for 7 electrons in eight-site $f c c$ bulk $^{31}$ and surface $^{32}$ clusters. The bulk calculation illustrates clearly the power of group-theoretical techniques, where a $1024 \times 1024$ matrix is diagonalized in closed form after being block-diagonalized. Recent work has concentrated on the square lattice at half-filling and with one or two holes. ${ }^{33}$ The cluster sizes are large (up to 18 sites) so only the low-lying eigenvalues and eigenvectors were determined.

The small-cluster approach has also been applied to the study of real materials. It is quite successful in describing properties that depend on short-range many-body correlations. These include photoemission in transition metals ${ }_{4}{ }^{34}$ alloy formation, ${ }^{35}$ surface photoemission ${ }^{36}$ in $\mathrm{Ni}$ and $\mathrm{Co}$, and surface magnetization ${ }^{37}$ in $\mathrm{Fe}$. This technique has also been applied to multi-band versions of the Hubbard model that describes high-temperature superconductivity in the $\mathrm{CuO}_{2}$ planes. ${ }^{38}$

In this contribution the-ground-state symmerry, $k$-vector, and spin are examined as a functions of electron concentration and interaction strength for the $t-f^{\prime}-J$ model on eight-site clusters for $s c, b c c, f c c$, and $s q$ lattices with PBC. In the next section the method of calculation is described; in Section III.3 the results for the ground-state properties, phase diagrams for regions of stability in parameter space, and ferromagnetic ground-state solutions are presented; in Section II.4 low-lying excitations is the many-body spectra are examined to determine regions in parameter space where HF behavior is expected; in the final section the conclusions and some conjectures are presented.

\section{III.2 Calculational Details}

The dimension of the Hamiltonian matrix grows exponentially with the size of the cluster (e,g., an $M$-site cluster with one orbital per site has dimension $\left.4^{M} \times 4^{M}\right)$. This rapid srowth restricts the maximum size of the cluster to be on the order of 10 sites. In the strong-interaction regime (i.e., the $t-t^{\prime}-J$ model), double-occupancy of an 
orbital is forbidden, reducing the Hilber space from $4^{M}$ to $3^{M}$ (for eight-site clusters this corresponds to an order of magnitude simplification from 65,536 to 6,561). The systematic use of conserved quantities and symmetries of the Hamiltonian provides further simplifications.

The total-number operator $N=\sum_{i, \sigma} n_{i \sigma}$ commutes with the Hamiltonian in Eq. (3.1) and is a conserved quantity. The Hilbert space with definite electron number $N$ reduces to dimension $2^{N} M ! / N !(M-N) !$ as summarized in Table 3.1 for the eightsite cluster. The largest remaining block size is now $1,792 \times 1,792$ for the 5 and 6 electron cases.

The electronic states can be further characterized by their spin and spatial symmetries. Since the total spin, the total z-componeri of spin, and the total spin raising and lowering operators all commute with the Hamiltonian, the many-body states may be labeled by the total spin $S$ and the total z-component of spin $m_{S}$, with every state in a given spin multiplet degenerate in energy. The spatial symmetry is labeled by the irreducible representation of the space group that transforms according to the manybody state. In our case, the space groups are symmorphic, moderaisly sized finite groups, that are constructed from the point group operations and the eight transiation vectors of the lattice (see the appenfix). The grand orthogonality theorem and the matrix element theorem ${ }^{39-41}$ (generalized Unsbld theorem) guarantee that the Hamiltonian matrix will be in block-diagonal form, with no mixing between states of different spin or spatial symmery, when it is expanded in a symmetrized basis that has definite spin and transforms according to the $(1,1)$ matrix elements of an irreducible representation of the space group. A symmetry-adapted computer algorithm was written that, given the lattice structure of a small cluster with PBC (see the appendix), the generators ${ }^{42}$ of the space group, and the character table $e^{43}$ of the space group, calculates the $(1,1)$ matrix elements of the irreducible representations (in a fashion similar to Lvehrmann $^{41}$ ). These matrix elements are used to construct projection operators that 
operate on maximum z-component of spin states $\left(m_{S}=S\right)$ to generate symmetrized basis functions of definite spin and spatial symmetry. The Hamiltonian blocks are determined in this symmetrized basis and are checked for completeness within each subspace of definite spin and spatial symmetry. The resultant blocks are diagonalized by the so-called QL algorithm ${ }^{44}$ which determines all of the eigenvalues and eigenvectors in the many-body problem. Table $\mathbf{3 . 2}$ summarizes the reduced block sizes for the four different lattices considered. The application of full spin and space group symmetry reduces the block sizes by another two orders of magninde which, in turn, reduces the diagonalization time by six orders of magnitude. This symmetry-adapted algorithm was tested for 7 electrons in an eight-site $f c c$ cluster and verified the known analytic results ${ }^{31}$ for that case.

The effect of geometry on the many-body solutions to the $t-t^{\prime}-J$ model is studied by solving the model exactly for four different crystalline environments: the $s c$, $b c c, f c c$, and $s q$ lattices. The eight-site clusters with PBC for these different structures are illustrated in real space and reciprocal space in Figs. 3.1-3.4. The PBC will renormalize the parameters in the Hamiltonian (3.1) when the summations in Eq. (3.2) are restricted to run over the finite cluster $(1 \leq i, j \leq 8)$. For example, the six nearestneighbors of an even [odd] site $i$ in the sc lattice (see Fig. 3.1) are two each of the odd [even] sites (excluding the site 9-i), the twelve next-nearest neighbors are four each of the remaining even [odd] sites, and the eight third-nearest neighbors are eight each of the site $9-i$. This renormalizes the parameters in the $t-t-J$ model by $t \rightarrow 2 t$, $t \rightarrow 4 t$, and $J \rightarrow 2 J$. Similar analysis for the other crystalline structures is given in Table 3.3.

The small-cluster approach samples the first Brillouin zone at eight $k$-vectors, which correspond to only three $(b c c, f c c)$ or four $(s c, s q)$ different symmetry stars. As summarized in Table 3.4, the one-electron energies of the small-cluster Hamiltonian agree precisely with the one-electron band structure of the infinite crystal, when 
sampled at the common $k$-vectors. Some of the properties of the many-body states can be understood by the naive picture of occupying these one-electron levels as if the electrons were noninteracting (see below).

The space groups that are relevant for totally symmetric orbitals on each site of the cluster have $48(s c), 192(b c c, f c c)$ or $64(s q)$ distinct elements. They are divided into $10(s c), 14(b c c), 13(f c c)$, and $16(s q)$ elasses, respectively. The character tables for these space groups are given in the appendix.

The nearest-neighbor hopping matrix element $|t|$ is chosen to be the unit of energy. Three different cases are examined for the next-nearest-neighbor hopping matrix element: $t^{\prime}>0, t^{\prime}=0$, and $t^{\prime}<0$. The magnitude of $t^{\prime}$ is chosen to be 0.5 for the $b c c$ lattice. This sets $\left|t^{\prime}\right|=0.15$ for the other three lattices, when exponential dependence of the hopping matrix elements on the distance between lattice sites is assumed.

Finally, note that whenever the lattice is bipartite $(s c, b c c, s q)-i . e .$, it can be separated into two sublattices $A$ and $B$ such that the nearest-neighbor hopping is $A \rightarrow B$ and $B \rightarrow A$ and the next-nearest-neighbot hopping is $A \rightarrow A$ and $B \rightarrow B$ only

- then the $t-t^{\prime}-J$ model has an eigenvalue spectrum that is symmetric ${ }^{16}$ in $t$. This allows the discussion to be limited ${ }^{45}$ to $t=1$ for the $s c, b c c$, and $s q$ latices; while both $t=1$ and $t=-1$ are considered for the $f c c$ lattice.

\section{III.3 Results: Ground State Symmetry}

The $k$-vector, spatial (small group of $k$ ) symmetry, and spin of the many-body ground state are calculated exactly for all elsctron fillings $(0 \leq N \leq 8)$ and for $0.0 \leq J<1.0$. The symmetry of the ground state is recorded by attaching the spinmultiplicity $(2 S+1)$ as a superscript to the symbol for the irreducible representation that transforms according to the many-body state (as given in the appendix). The $t-t^{\prime}-J$ model on small clusters has many accidental degeneracies; that is, degeneracies 
that are not required by the spin and space-group symmetries of the underlying latrice (see below). Some of these degeneracies are inherent in the model itself, ${ }^{7.23}$ while other degeneracies occur due to finite-size effects ${ }^{46}$ (permutation symmetries of the small cluster that are not representable as space group symmetries; see Chapter V for a more complete discussion).

The cases of low electron filling $(N \leq 3)$ are well-described by occupying the lowest one-electron energy levels (Table 3.4). These one-tectron energy levels have a rich structure. The lowest level is nondegenerate and has $\Gamma_{1}$ symmetry for the sc, $b c c, f c c(t>0)$, and $s q$ lattices, while the lowest level for the $f c c(t<0)$ lattice is threefold degenerate with $X_{1}$ symmery. The first excited level is threefold $\left(X_{1}\right)$, sixfold $\left(N_{1}\right)$, or fourfold $\left(L_{1}\right)$ degenerate for the $s c, b c c$, and $f c c$ lattices, respectively. The sq lattice does not have a unique first excited level: when the $2 N N$ hopping integral vanishes $\left(t^{\prime}=0\right)$ there is an accidental degeneracy of $\Sigma_{1}$ and $X_{1}$, creating a sixfold degenerate level; for $t>0$ the ordering is $\Sigma_{1}$ (fourfold degenerate) $<X_{1}$ (twofold degenerate), and vice versa for $t<0$.

Since the case of one electron $(N=1)$ contains no many-body effects, the ground state is formed by occupying the lowest one-electron level. The ground state, therefore, has symmetry ${ }^{2} \Gamma_{1}(d=2)$ for the $s c, b c c, f c c(t>0)$, and $s q$ latices and ${ }^{2} X_{1}(d=6)$ for the $f c c(t<0)$ lattice. A second electron $(N=2)$ is added by placing $^{15}$ it in a spin-singlet state in the same level as the first electron. This results in a ${ }^{\mathrm{t}} \Gamma_{1}(d=1)$ symmetry for the ground state of the $s c, b c c, f c c(t>0)$, and $s q$ lattices. The $f c c(t<0)$ lattice has ${ }^{1} \Gamma_{12}(d=2)$ symmetry for finite $J$, but has a spindegenerate ${ }^{3} X_{2} \oplus^{1} \Gamma_{12}(d=11)$ ground state when $J=0$ (because of the degeneracy of the one-electron levels).

In general, the addition of a third electron $(N=3)$ is made by placing it in the first-excited one-electron energy level. This yields a ${ }^{2} X_{1}(d=6),{ }^{2} N_{1}(d=12)$, and ${ }^{2} L_{1}(d=8)$ ground state for the $s c, b c c$, and $f c c(t>0)$ lattices. The $s g$ latice has a 
${ }^{2} \Sigma_{1}(d=8),{ }^{2} X_{1} \oplus{ }^{2} \Sigma_{1}(d=12)$, or ${ }^{2} X_{1}(d=4)$ ground state for $t^{\prime}>0, t=0$, and $t<0$ respecrively. However, many-body effects begin to play a more important role in the three-electron case. There is a level crossing in the $s c$ ground state from ${ }^{2} X_{1}(d=6)$ to ${ }^{2} \Gamma_{12}(d=4)$ at $J / t=0.85100$ when $t^{\prime}<0$. The $f c c(t<0)$ case is even more interesting. It is the first example of a ferromagnetic ground state ${ }^{4} \Gamma_{1}(d=4)$ (resulting from the application of Hund's empirical rule ${ }^{47}$ ) which undergoes a level crossing to a spin-doublet ${ }^{2} X_{2}(d=6)$ at $J / t=0.29972\left(t^{\prime}>0\right), J / t=0.23617\left(t^{\prime}=0\right)$, or $J / t=0.15045\left(t^{\prime}<0\right)$.

Many-body effects become increasingly more important for $N \geq 4$. The groundstate symmetries are recorded in Tables 3.5-3.8 for the cases $4 \leq N \leq 7$.

The half-filled band $(N=8)$ reduces to the case ${ }^{8}$ of a Heisenberg antiferromagnet. The solutions are all spin-singlets, have symmetry ${ }^{1} \Gamma_{1}(d=1)$ for the $s c$, bcc, and $s q$ lattices, and have ${ }^{1} \Gamma_{1} \oplus^{1} \Gamma_{12}(d=3)$ symmetry for the $f c c$ lattices.

The results agree with previous work for the $s c$ latrice, ${ }^{25-27}$ the $f c c$ lattice, ${ }^{31}$ and the sq latrice. ${ }^{48}$ There are a few salient fearures of thsse results that deserve comment:

(a) The case of the sq lattice with $t^{\prime}=0$ is identical to the $b c c$ lattice with $t^{\prime}=0$ due to a hidden symmetry of the eight-site sq lattice.

(b) There is a large number of ferromagnetic ${ }^{49}$ solutions for $J \ll t$. These solutions occur in the sc latrice $\left(t^{\prime} \leq 0 . N=4 ; t^{\prime} \leq 0, N=7\right)$, in the bcc lattice $\left(t^{\prime} \leq 0, N=7\right)$, in the $f c c(t<0)$ lattice (all $t^{\prime}, N=3$; all $t, N=7$ ), and in the $s q$ lattice (all $t^{\prime}, N=7$ ). The ferromagnetic solutions for $N=7$ are all examples of Nagaoka's theorem ${ }^{24}$ for one hole in a half-filled band at $J=0$.

(c) There is also a large number of ferrimagnetic ${ }^{50}$ solutions for $J \ll t$. When $N=4$, ferrimagnetic solutions occur for all geometries except the sc lattice; when $N=5$, ferrimagnetism occurs in the sc and $f c c(t>0)$ lattices; and when $N=7$, it occurs for all lattices except $f c c(t>0)$. 
(d) Whenever the Heisenberg interaction $J$ is large enough, the ground state is stabiljzed in the lowest spin configuration ( $S=0$ for $N=$ even, $S=1 / 2$ for $N=$ odd). In particular, the case of two holes $(N=6)$ is always a spin-singlet.

(e) Non-minimal-spin solutions undergo "spin-cascade" transitions, passing through each intermediate spin en route to minimal spin solutions, as $J$ is increased. The only exceptions are in the sc lattice $(t \leq 0, N=4$ and $t=0, N=7)$ which have one level crossing from maximal spin to minimal spin and the sq lattice $\left(t^{\prime}<0, N=7\right)$ which does not have a spin-5/2 ground state in the cascade from spin-7/2 to spin-1/2.

(f) Ground states that are accidentally degenerate for all values of $J$ always have the same total spin, but usually have space symmetries corresponding to different $k$ vectors. The $s c$ lattice is the only cluster that has no "accidental" degeneracies in the ground state.

(g) At $J=0$ there are some solutions with additional accidental degeneracies. The degenerate states contain mixtures of different total spin. These special solutions are summarized in Table 3.9.

There are many magnetic solutions to the $t-t-J$ model. Hund's empirical rules ${ }^{47}$ may be employed to explain the occurence of ferrimagnetism for $N=4$ and $N=5$, but, as the filling increases, many-body effects overwhelm the system and the one-electron picture loses its predictive power. The $N=7$ cases verify Nagaoka's theorem, ${ }^{\text {I4 }}$ but the ferromagnetic state is quite unstable with respect to the interaction parameter $J$, with a rapid level crossing to a lower spin state. Geometry also plays a role, as the $s c$ and $f c c$ lattices exhibit far stronger magnetic properties than the bcc or $s q$ lattices.

The case with two holes $(N=6)$ has a spin-quenched ground state for all four different geometries. This fact has been observed for many geometries by previous investigators in small cluster calculations. ${ }^{25-27,29-31,33}$ There are also variational and 
heuristic arguments why the two-hole state cannot be ferromagnetic. ${ }^{17,51}$ Our solutions (Table 3.7) show one interesting additional feature: The ground-state manifold always contains a state with ${ }^{1} \Gamma_{1}$ symmetry whenever the hypotheses of Nagaoka's theorem ${ }^{14}$ are satisfied $(t \leq 0$ for the $s c, b c c, f c c(t<0)$, and sq latrices). This result suggests that there is a two-hole extension to Nagaoka's theorem which yields a spir-singlet ground staie. This result is left as a conjecture, however, and no proof is offered.

Up to this point the electron occupation number $N$ has been fixed. It is important, however, to examine the stability of a fixed- $N$ ground state with respect to disproportiation (a macroscopic rearrangement of the crystal into domains, with different electron number in each domain, but with an average filling $N$ ). The stability of a particular ground state (for fixed interaction $J$ ) is determined by forming the convex hull of the ground-state energy versus electron filling and comparing it to the calculated ground-state energy for $N$ electrons. If the convex hull is lower in energy, then the ground state with $N$ electrons is unstable against disproportiation. Previous work ${ }^{33}$ on the phenomenon of disproportiation has concentrated exclusively on one and two holes in the half-filled band of a square lattice (determining the binding energy of hole pairs).

Alternatively, one can view the small cluster with $P B C$ as an approximation to an infinite crystal constructed from a repetition of the small-cluster units. The trial energy for a variational wavefunction (built out of produets of many-body wavefunctions for each small-cluster unit) lies on the convex hull for a given electron concentration. Therefore, the stability against disproportiation can be interpreted as the simplest possible extrapolation of the small-cluster wavefunctions (neglecting any "interference effects") to the thermodynamic limit.

The resules are summarized in the form of phase diagrams (Figs. 3.5-3.9). The phase diagrams plot regions of parameter space that are stable against disproportiation 
as functions of the electron filling $N$ (vertical axis) and the Heisenberg interaction $J$ (horizontal axis). Horizontal solid lines denote stable ground-state solutions for fixed $N$. Dotted vertical lines separate regions where disproportiation occurs and also denote regions where the ground state for fixed $N$ has a level crossing (see Tables 3.5-3.8). The level crossings for fixed $N$ are also marked by a solid dot in the phase diagrams.

In general, the tendency toward disproportiation increases as the interaction $J$ increases, however, there are cases where islands of stable ground-state configurations form (these include $N=4$ in the bcc, $f c c(t<0)$, and $s q$ latrices, and $N=7$ in the $f c c(t<0)$ latrice). The role of geometry on the structure of the phase diagrams can be explained by three empirical rules (listed in order of importance): (1) ground-state solutions with even numbers of electrons are more stable than solutions with odd numbers of electrons (in particular, $N=0, N=2$, and $N=8$ are always stable); filled or half-filled one-electron shells are stable in relation to other electron fillings; and (3) when the ground state foi an odd number of electrons $(N)$ is stable, the ground states for even numbers of electrons $(N \pm 1)$ are also stable. In particular, the third sule implies that whenever an ( $N=$ odd) solution becomes unstable with respect to disproportiation, it always separates into even mixtures of solutions with $(N \pm 1)$ electrons. However, when an $(N=$ even) solution becomes unstable, it separates into many different kinds of mixtures $(N \pm 2 ; N+2, N-4 ; N+2, N-1 ; N+4, N-2)$. These empirical rules explain the stability of $N=0,1,2,4,5,6,8$ for the se lattice; $N=0,1,2,8$ for the bce and $s q\left(l^{\prime}=0\right)$ lattices; $N=0,1,2,6,8$ for the $f c c(t>0)$ and $s q(t>0)$ lattices; $N=0,2,3,4,6,8$ for the $f c c(t<0)$ lattice; and $N=0,1,2,4,8$ for the $s q\left(l^{\prime}<0\right)$ lattice. The nules do not explain the stability of $N=6,7$ in the bcc lartice or $N=7$ in the $f c c(t<0)$ lattice. We believe the last feature arises from many-body effects and a sensitivity of the solutions to the nextnearest-neighbor hopping $r$. The $b c c$ and $f c c(t<0)$ lattices are strongly sensidive to 
$t$, the sq lattice is moderately sensitive to $t$, and the $s c$ and $f c c(t>0)$ lattices are insensitive to $t$. Finally, note that although the sq lattice does show regions of parameter space which favor pair-formation of holes in the half-filled band, it is the $f c c$ lattice with $t>0[t<0]$ that shows the strongest tendency toward hole [electron] pair-formation in the $t-t^{\prime}-J$ model. This result suggests that frustration is a key element for stable pair-formation in itinerant interacting electronic systems and that the $f c c$ lattice is more likely to be superconducting than the sq lattice for a single-band model.

\section{III.4 Results: Heavy-Fermion Behavior}

Two electrons which have strong correlation (i.e., satisfy the "super" Pauli principle) must avoid each other when moving in a solid. This places an additional constraint on the electron dynamics which should, in turn, strongly affect the transport properties and the density of states at the Fermi level; e.g. Jeduce the specific heat, electron conductivity, etc. The constraint is felt in many-body solutions by a drastic reduction in the number of available states (reduced by one order of magnitude in eight-site clusters). Under certain circumstances, however, some properties are enhanced by orders of magnitude because of strong correlation (as evidenced in the HF materials ${ }^{2,3}$ ). Analogous behavior is found in the many-body solutions to the $t-t-J$ model on small clusters.

The HF materials exhibit large coefficients of the term linear in the temperature in their specific heat, quasi-elastic magnetic excitations (large magnetic susceptibilidies), and poor metallic conductivity. The solutions to the $t-r^{\prime}-J$ model are tested to find candidate solutions that depict this HF behavior. Since electron correlation effects begin to be large at $N=4$, it is expected that solutions near half-filling will have the strongest HF character. 
The large coefficient of the linear term in the specific heat arises from an abundance of low-lying excitations, i.e. many-body states in the ground-state manifold and energetically close to it. We calculated the maximum number of states (including all degeneracies) lying within an energy of $0.1|t|$ of the ground state for $0.0 \leq J<1.0$ (see Table 3.10). The maximum number of states appear for only a finite range of $J$, as illustrated for the three cases in Figs. 3.10-3.12. Enhancements ${ }^{52}$ are searched for in the strong-correlation regime by comparing the maximum number of states in Table 3.10 with the total number of states in the ground-state manifold of the noninteracting regime (Table 3.11). The degeneracy of the noninteracting ground-state manifold is determined by a paramagnetic ${ }^{53}$ filling of the one-electron levels of Table 3.4 (all of the excited states in the noninteracting electron spectrum lie beyond $0.1|t|$ of the ground state). The possible HF lie predominantly near half-filling and are highlighted in boldface in Table 3.10. Both the bcc and the sq $(t=0)$ lattices show no tendency toward HF behavior due, in part, to the large density of states of the noninteracting electrons at half-filling.

Large magnetic susceptibilities and large magnetic fluctuations accur whenever two states with different total spin are nearly degenerate. These fluctuations increase when more than two different total-spin configurations are nearly degenerate (a feature that we call the spin-pileup effect). Many soluvions exhibit this spin-pileup effect: the case of a half-filled band has, for all structures, five different total-spin configurations degenerate at $J=0$; for $N=7$, the spin-pileup effect is seen in the sc $\left(t^{\prime} \leq 0\right)$, $b c c\left(t^{\prime}=0\right), f c c(t>0)$, and sq lattices; for $N=6$ and $N=4$ it is observed in the $f c c(t>0)$ lattice and in the sc $\left(r^{\prime}=0\right)$ lattice, respectively. A simple example of the spin-pileup effect is illustrated in Fig. 3.11. Cases when only two different total-spin states are nearly degenerate occur in the regions near isolated level crossings between the two states. These regions have been summarized in Tables 3.5-3.8. 
Finally, the candidate HF solution: are required to exhibit weak metallic conductivity. Previous investigations ${ }^{31}$ hrive shown that electrons in the strongly correlated regime are poor conductors (in particular, the half-filled band has electrons that are frozen in space, i.e. an insulator). However, an enhancement of the conductivity is expected whenever a solution is close to a disproportiation instability, since the system has states with two different charge distributions which are nearly degenerate.

Solutions to the $t-t^{\prime}-J$ model that satisfy all three criteria ${ }^{54}$ are the best candidates for models of HF systems. These solutions are listed in Table 3.12. The solutions lie predominantly near half-filling, are quite sensitive to variations in $J$, are moderately sensitive to changes in $r$, and may be magnetic. In fact, the geometrical tendency toward HF appears to be closely linked to the geometrical tendericy toward magnetism of the previous section, with the $s c$ and $f c c$ lattices having stronger HF character than the $b c c$ and $s q$ latices.

\section{III.5 Conclusions}

The effect of geometry on the exact many-body solutions of the $t-t^{\prime}-J$ model in eight-site small clusters has been studied. Five particular cases were examined: sc; $b c c ; f c c(t>0) ; f c c(t<0)$; and $s q$ lattices. Spin and space-group symmetries were used to reduce the Hamiltonian to block-diagonal form, which decreased the diagonalization time by six orders of magninde.

The spatial symmetry, $k$-vector, and total spin of the ground state were calculated for all electron fillings as a function of the interaction strength. It was found that the ground state typically has minimal spin and there are many accidental degeneracies. Magnetic solutions (including ferromagnetism) occur in some cases when $J \propto 1$. In particular, Nagaoka's theorem ${ }^{14}$ was verified, the ferromagnetic solutions were found to be quite unstable wiln respect to increasing $J$, and an extension of the theorem to the case of two holes was proposed: Whenever the hypotheses of Nagaoka's 
theorem ${ }^{14}$ are satisfied and there are exactly two holes in the half-filled band, then the ground-state manifold includes a spin-singlet with. ${ }^{1} \Gamma_{1}$ symmetry. This conjectured extension of Nagaoka's theorem indicates that the ferromagnetic solution is quite unstable to both interaction strength $J$ and electron filling $N$.

The stability of the many-body solutions with respect to disproportiation was studied. Amazingly enough, it was found that the phase diagrams can be almost entirely described by a one-electron picture: The stability of solutions tends to decrease as the interaction $J$ is increased; the one-eighth $(N=2)$ and one-half $(N=8)$ filled bands are always stable; an even number of electrons tends to be more stable than an odd number; and an odd number of electrons that forms a half-filled one-electron shell tends to be stable. Frustration was a key element to the binding of two holes or two electrons, as shown in the $f c c$ lattice. In particular, no evidence was found for enhanced superconductivity (via the binding of holes) in the two-dimensional sq lattice versus the three-dimensional latices.

Heavy-fermion behavior was studied by examining the character of the groundstate manifold and its low-lying excitations. Many-body solutions were found that have a large density of many-body states near the ground state, have large spin fluctuations, and are poor metallic conductors. These solutions exhibit HF character for only a small range of the interaction and are sometirnes magnetic.

Geomery plays a similar role in both magnetism and HF behavior. The sc and $f c c$ lattices have a stronger tendency toward magnetism and HF behavior than the bcc and $s q$ lattices.

In conclusion, the small-cluster technique is an alternate approach to the manybody problem that treats electron correlation effects exactly, but has uncontrolled finite-size effects. Group theory is used to simplify the problem, so that many different cases can be studied. A richness to the stucture of the ground-state solutions as functions of the interaction strength, electron flling, and geometry, is found that 
includes magnetism and $\mathrm{HF}$ behavior.

\section{II.6 Appendix: Space-Group Symmetry of Eight-Site Clusters}

The cubic point group $O_{h}$ has 48 operations, however, the improper rotations and inversion yield no additional information when spherically symmetric orbitals are placed at each lattice site. Therefore, the relevant cubic point group for the small clusters that are studied is the octahedral group $O$ which has 24 operations. Similarly, the relevant point group for the square lattice is $C_{4 v}$ which has 8 operations. The eightsite cluster has eight translations which yield space groups of order 192 [64] for the cubic [square] latices. However, it turns out that there is a fourfold redundancy of group operations in the sc lattice when spherically symmerric orbitals are placed at the lattice sites (a similar phenomena occurs in the four-site tetrahedral cluster ${ }^{28}$ ). This reduces the order of the space group for the sc cluster to 48 and this reduced group is isomorphic to the point group $O_{h}$ with an origin at the center of the cube (see Chapter v).

The sc Brillouin zone ${ }^{43}$ is sampled at four symmetry stars: $\Gamma(\mathrm{d}=1) ; R(\mathrm{~d}=1) ; M$ $(d=3)$; and $X(d=3)$. The character table ${ }^{43}$ is reproduced in Table 3.13 with the conventional and the space group notations for the 10 irreducible representations.

The $b c c$ and $f c c$ lattices display the full symmetry of the proper space gre?Their Brillouin zones ${ }^{43}$ are sampled at three symmetry stars: $\Gamma(\mathrm{d}=\mathrm{i}) ; H(\mathrm{~d}=1)$; and $N$ $(d=6)$ for the bce lattice and $\Gamma(d=1) ; X(d=3)$; and $L(d=4)$ for the $f c c$ latrice. The character tables ${ }^{43}$ are reproduced in Tables 3.14 and 3.15. The space group operations are denoted by a point group operation (with origin at site 1) and a translation vector. Nearest-neighbor translations are denoted by $\tau$ and next-nearest neighbor translations by $\theta$. The subseripts 11,1 , and $L$ refer to translations that are parallel to, perpendicular to, or at an angle to the rotation axis of the point group operation. 
The sq lattice also displays the full symmetry of the proper space group. The Brillouin zone $\mathrm{e}^{43}$ is sampled at four symmetry stars: $\Gamma(\mathrm{d}=1) ; M(\mathrm{~d}=1) ; X(\mathrm{~d}=2)$; and $\Sigma$ $(d=4)$. The character table ${ }^{43}$ is reproduced in Table 3.16. The symbol $\sigma$ denotes reflections in the planes perpendicular to the $x$ - and $y$-axes, $\sigma$ denotes reflections in planes perpendicular to the diagonals $x \pm y, \Omega$ denotes the third-nearest-neighbor translations, and the subscripts $\|(1)$ refer to translations that are parallel (perpendicular) to the normal of the mirror plane.

Finally, we elaborate upon the algebraic identification of the lattice points in an infinite lattice with those of an eight-site cluster with PBC. A sc lattice is described by triples of integers $(i, j, k)$. The eight-site sc cluster with $\mathrm{PBC}$ describes the same set of points, but each point on the infinite lattice is identified with one of eight equivalence classes, determined by the site in the small cluster with which it is equivalent. These equivalence classes are given in Table 3.17 for the $s c, b c c, f c c$, and $s q$ lattices. 


\section{III.7 References for Chapter III}

1 R. M. White, Quannum Theory of Magnetism, (McGraw-Hill, New York, 1970).

2 G.R. Stewart, Rev. Mod. Phys. 56, 755 (1984).

3 P. Fulde, J. Keller, and G. Zwicknagl, in Solid Stare Phys., edited by H. Ehrenreich, F. Seitz and D. Tumbull (Academic, New York, 1988), Vol. 41, p. 1.

4 J.G. Bednorz and K.A. Muller, Z. Phys. B 64, 189 (1986); M.K. Wu, J.R. Ashburn, C.J. Torng, P.H. Hor, R.L. Meng, L. Gao, Z.J. Huang, Y.Q Wang, and C.W. Chu, Phys. Rev. Lett. 58, 908 (1987).

5 P.W. Anderson, Science 235, 1196 (1987); C.M. Varma, S. Schmitt-Rink, and E. Abrahams, Solid State Commun. 62, 681 (1997); V.J. Emery, Phys. Rev. Lett. 58, 2794 (1987); F.C. Zhang and T.M. Rice, Phys. Rev. B 37, 3759 (1988).

6 The summations in Eq. (3.2) run over all nearest-neighbor $i, j$ (i.e., each pair is counted twice) to guarantee hermiticity of the Hamiltonian.

7 J. Hubbard, Proc. R. Soc. London, Ser. A 276, 238 (1963); ibid., 277, 237 (1964); ibia., 281, 401 (1964); ibid., 285, 542 (1965); ibid., 296, 82 (1967); ibid., 296, 100 (1967).

8 P.W. Anderson, in Solid Stase Physics, edited by F. Seizz and D. Tumbull (Academic, New York, 1963), Vol. 14, p. 99.

9 J.R. Schrieffer and P.A. Wolff, Phys. Rev. 149, 491 (1966).

10 A.B. Harris and R.V. Lange, Phys. Rev. 157, 295 (1967).

11 A.H. MacDonald, S.M. Girvin, and D. Yoshioka, Phys. Rev. B 37, 9753 (1988); A.M. Oles, ibid., 41, 2562 (1990); A.H. MacDonald, S.M. Girvin, and D. Yoshioka, ibid., 41, 2565 (1990).

12 W. Marshall, Proc. R. S $\propto$. London, Ser. A 232, 48 (1955); E.H. Lieb and D.C. Mattis, J. Math. Phys. 3, 749 (1962). 
E.H. Lieb, Phys. Rev. Lett. 62, 1201 (1989).

Y. Nagaoka, Solid State Commun. 3, 409 (1965); D.J. Thouless, Proc. Phys. Soc. London 86, 893 (1965); Y. Nagaoka, Phys. Rev. 147, 392 (1966); H. Tasaki, Phys. Rev. B 40, 9192 (1989).

E.H. Lieb and D.C. Martis, Phys. Rev. 125, 164 (1962).

E.H. Lieb and F.Y. Wu, Phys. Rev. Lett. 20, 1445 (1968).

B. Doucpt and X.G. Wen, Phys, Rev. B 40, 2719 (1989).

M. Ogata and H. Shiba, Phys. Rev. B 41, 2326 (1990).

B. Sutherland, Phys. Rev. B 12, 3795 (1975); P. Schlorman, ibid., 36, 5177 (1987); ibid., 41, 4164 (1990); M. Kotrla, Phys. Lett. A 145, 33 (1990); P.A. Bares and G. Blatter, Phys. Rev. Lett. 64, 2567 (1990).

For a review see L.M. Falicov in Recent Progress in Many-Body Theories, edited by A.J. Kallio, E. Pajanne, and R.F. Bishop (Plenum, New York, 1988), Vol. 1, p. 275; J. Callaway, Physica B 149, 17 (1988).

L.M. Falicov, Group Theory and Its Physical Applicarions, (University of Chicago, Chicago, 1966) p. 144ff.

L.M. Falicov and R.A. Harris, J. Chem. Phys. 51, 3153 (1969).

O.J. Heilmann and E.H. Lieb, Trans. NY Acad. Sci. 33, 116 (1971).

H. Shiba and P.A. Pincus, Phys. Rev. B 5, 1966 (1972); K.-H. Heinig and J. Monecke, Phys. Stat. Sol. B 49, K139 (1972); ibid. 49, K141 (1972); ibid. 50, K1 17 (1972); H. Shiba, Prog. Theor. Phys. 48, 2171 (1972); D. Cabib and T.A. Kaplan, Phys. Rev. B 7, 2199 (1973).

M. Takahashi, Phys. Soc. Japan 47, 47 (1979).

A. Kawabata, Solid State Commun. 32, 893 (1979); A. Kawabata in Electron Correlation and Magnetism in Narrow Band Systems, edited by T. Moriya, (Springer, New York, 1981) p. 172. 
J. Callaway, D.P. Chen, and Y. Zhang, Phys. Rev. B 36, 2084 (1987).

A.M. Oleś, B. Oles, and K.A. Chao, J. Phys. C 13, L979 (1980); J. Rössler, B. Fermandez, and M. Kiwi, Phys. Rev. B 24, 5299 (1981); D.J. Newman, K.S. Chan, and B. Ng, J. Phys. Chem. Solids 45, 643 (1984); L.M. Falicov and R.H. Victora, Phys. Rev. B 30, 1695 (1984); C.M. Willet and W.-H. Steeb, J. Phys. Soc. Japan 59, 393 (1990).

M. Takahashi, J. Phys. Soc. Japan 51, 3475 (1982).

Y. Ishii and S. Sugano, J. Phys. Soc. Japan 53, 3895 (1984); J. Callaway, D.P. Chen, and R. Tang, J. Phys. D 3, 91 (1986); Phys. Rev, B 35, 3705 (1987); J. Callaway, ibid., 35, 8723 (1987); W. Pesch, H. Bluttner, and J. Reichl, ibid., 37, 5887 (1988); J. Callaway, D.P. Chen, Q. Li, D.G. Kanhere, and P. Misra, (unpublished); J. Callaway, D.P. Chen, D.G. Kanhere, and Q.Li, (unpublished).

A. Reich and L.M. Falicov, Phys. Rev. B 37, 5560 (1988); ibid., 38, 11199 (1988).

C. Chen, A. Reich, and L.M. Falicov, Phys. Rev. B 38, 12823 (1988); C. Chen, Phys. Lett. A 142, 271 (1989).

E. Kaxiras and E. Manousakis, Phys. Rev. B 37, 656 (1988); X. Zotos, ibid., 37, 5594 (1988); E. Kaxiras and E. Manousakis, ibid., 38, 866 (1988); J. Bonca, P. Prelovsek, and I. Sega, ibid., 39, 7074 (1989); J.A. Riera and A.P. Young, ibid., 39, 9697 (1989); ibid., 40, 5285 (1989); E. Dagoto, A. Moreo, and T. Barnes, ibid., 40, 6721 (1989); Y. Fang, A.E. Ruckenstein, E. Dagotto, and S. SchmittRink, ibid. 40, 7406 (1989); Y. Hasegawa and D. Poilblanc, ibid., 40, 9035 (1989); Int. J. Mod. Phys. B 3, 1853 (1989); A. Parola, S. Sorella, S, Baroni, M. Parrinello, and E. Tosatti, ibid., 3, 139 (1989); M. Imada and Y. Hatsugai, J. Phys. Soc. Japan 58, 3752, (1989); E. Dagotto, A. Morec. R.L. Sugar, and D. Toussaint, Phys. Rev. B 41, 811 (1990); D. Poilblanc, Y. Hasegawa, and T.M. Rice, ibid., 41, 1949 (1990); K.-H. Luk and D.L. Cox, ibid., 41, 4456 (1990); V. 
Elser, D.A. Huse, B.I. Schraiman, and E.D. Siggia, ibid., 41, 6715 (1990); D. Poilblanc and Y. Hasegawa, ibid., 41, 6989 (1990).

34 R. H. Victora and L.M. Falicov, Phys. Rev. Lett. 55, 1140 (1985); E.C. Sowa and L.M. Falicov, Phys. Rev. B 35, 3765 (1987).

A. Reich and L.M. Falicov, Phys. Rev. B 37, 5560 (1988); E.C. Sowa and L.M. Falicov, ibid., 37, 8707 (1988).

36 C. Chen and L.M. Falicov, Phys. Rev. B 40, 3560 (1989); C. Chen, Phys. Rev. Lett. 64, $2176(1990)$.

C. Chen, Phys. Rev. B 41, 1320 (1990).

M. Ogata and H. Shiba, J. Phys. Soc. Japan, 57, 3074 (1988); H. Shiba and M. Ogata, J. Magn. and Magn. Mat. 76/77, 59 (1988); W.H. Stephan, w. v.d. Linden and P. Horsch, Phys. Rev. B 39, 2924 (1989); M. Ogata and H. Shiba J. Phys. Soc. Japan 58, 2836 (1989); Physica C 158, 355 (1989); D.M. Frenkel, R.J. Gooding, B.I. Shraiman, E.D. Siggia, Phys. Rev. B 41, 350 (1990); M.S. Hybersen, E.B. Stechel, M. Schllter, and D.R. Jennison, Phys. Rev. B 41, 11068 (1990).

M. Tinkham, Group Theory and Quantum Mechanics, (McGraw-Hill, New York, 1964) p. 20ff.; p. $80 f$.

40 L.M. Falicov, Group Theory and Its Physical Applications, (University of Chicago, Chicago, 1966) p. 20ff.; p. $46 \mathrm{ff}$.

41 A.W. Luehmann, Adv. in Phys. 17, 1 (1968).

G. Burns and A.M. Glazer, Space Groups for Solid State Scientises, (Academic, New York, 1978) p. 213ff.

L.P. Bouckaert, R. Smoluchowski, and E. Wigner, Phys. Rev. 50, 58 (1936).

J.H. Wilkinson and C. Reinsch, Handbook for Automatic Computation, Vol. II: Linear Algebra, edired by F.L. Bauer, (Springer-Verlag, New York, 1971) p. 227. 
45 Although the eigenvalue spectra is s:mmetric with respect to the sign of $t$, the symmery label for many-body states with odd numbers of electrons may depend on the sign of $t$.

46 T. Oguchi and H. Kitatani, J. Phys. Soc. Japan 58, 1403 (1989); R. Saito, Solid State Commun. 72, 517 (1989).

47 F. Hund, Linienspektren und Periodisches System der Elemente, (Springer, Berlin, 1927) p. 124; E.U. Condon and G.H. Shorley, The Theory of Atomic Spectra, (Cambridge University, Cambridge, 1957) p. 209.

48 The results agree with the work of Riera and Young in Ref. 32 except that in the $N=6$ case the spin-2 state is found to be lower in energy than the spin- 1 state (rather than degenerate in energy) when $J=0$.

49 Solutions with maximum spin are denoted as ferromagnetic.

5o Solutions with neither maximal nor minimal spin are denoted as ferrimagnetic, regardless of the spin-spin correlation functions (which have not been computed).

51 P.W. Anderson, B.S. Shastry, and D. Hristopulos, Phys. Rev. B 40, 8939 (1989); B. Doucot and R. Rammal, Int J. Mod. Phys. B 3, 1755 (1989); B.S. Shastry, H.R. Krishnamurthy, and P.W. Anderson, Phys. Rev. B 41, 2375 (1990); A. Basile and V. Elser, Phys. Rev. B 41, 4842 (1990).

52 There is a finite-size effect that can be very strong in the noninteracting case - it occurs whenever a one-electron shell is filled. The noninteracting solution is then an insulator with only one state in the ground-state manifold. In this case (and in the fillings near the closed shell) the strongly correlated solutions will undoubtedly be HF. These potential HF are not excluded unless they fail to satisfy the additional criteria.

53 Comparisons can be made with respect to ferromagnetic filling of the one-electron levels (which spans a Hilber space with the same dimension as the strongly 
correlated Hilbert space) but none of the HF are eliminated from such a comparison (the finite-size effect is much stronger in this case).

54 The HF with $N=3$ is near a disproportiation instability and may be too good a conductor. The HF with $N=4$ is stable against disproportiation but is probably metallic since strong-correlation effects are not expected to reduce drastically the electronic conductivity in a quarter-filled band. Neither solution is discarded. 
III.8 Tables for Chapter III

Table 3.1. Hamiltonian block sizes for $N$ electrons in an eight-site cluster in the strongly interacting limit (no double-occupation of a lattice site).

\begin{tabular}{cr}
\hline \hline & $\begin{array}{r}\text { Dimension of } \\
N\end{array}$ \\
\hline 0 & Hilbert Space \\
\hline 1 & 1 \\
2 & 16 \\
3 & 112 \\
4 & 448 \\
5 & 1120 \\
6 & 1792 \\
7 & 1792 \\
8 & 256 \\
\hline \hline
\end{tabular}


Table 3.2. Largest Hamilionian block sizes for $N$ electrons in the four different eightsite clusters when expanded in a symmetrized basis of definite spin and spatial symmetry.

\begin{tabular}{l|r|r|r|r}
\hline \hline$N$ & $s c$ & $b c c$ & $f c c$ & $s q$ \\
\hline 0 & 1 & 1 & 1 & 1 \\
1 & 1 & 1 & 1 & 1 \\
2 & 3 & 2 & 2 & 3 \\
3 & 8 & 5 & 5 & 8 \\
4 & 16 & 8 & 9 & 14 \\
5 & 18 & 9 & 12 & 18 \\
6 & 18 & 9 & 11 & 18 \\
7 & 8 & 5 & 5 & 8 \\
8 & 3 & 2 & 2 & 3 \\
\hline \hline
\end{tabular}


Table 3.3. Renormalized parameters for the $t-t^{\prime}-J$ Hamiltonizn when restricted to isolated eight-site lattices.

\begin{tabular}{c|c|c|c|c}
\multicolumn{7}{c}{$s c$} & $b c c$ & $f c c$ & $s q$ \\
\hline $1 \mathrm{NN}$ & $2 t$ & $2 t$ & $2 t$ & $t$ \\
$2 \mathrm{NN}$ & $4 t^{\prime}$ & $2 t^{\prime}$ & $6 t^{\prime}$ & $2 t$ \\
int & $2 J$ & $2 J$ & $2 J$ & $I$ \\
\hline
\end{tabular}


Table 3.4. One-electron energy levels for the four different eight-site clusters.

\begin{tabular}{l|l|l|l}
\hline \multicolumn{1}{c}{$s c$} & \multicolumn{1}{c}{$b c c$} & \multicolumn{1}{c}{$f c c$} & \multicolumn{1}{c}{$s q$} \\
\hline$E_{\Gamma}=-6 t-12 t^{\prime}$ & $E_{\Gamma}=-8 t-6 t^{\prime}$ & $E_{\Gamma}=-12 t-6 t^{\prime}$ & $E_{\Gamma}=-4 t-4 t^{\prime}$ \\
$E_{X}=-2 t+4 t^{\prime}$ & $E_{N^{\prime}}=2 t^{\prime}$ & $E_{L}=6 t^{\prime}$ & $E_{\Sigma}=0$ \\
$E_{M}=2 t+4 t^{\prime}$ & $E_{H}=8 t-6 t^{\prime}$ & $E_{X}=4 t-6 t^{\prime}$ & $E_{X}=4 t^{\prime}$ \\
$E_{R}=6 t-12 t^{\prime}$ & & & $E_{M}=4 t-4 t^{\prime}$ \\
\hline
\end{tabular}


Table 3.5. Ground-state symmetry for the $N=4$ case. The symmetry labels refer to the irreducible representations of the space group (see the appondix) with the superscript corresponding to the spin-degeneracy $(2 S+1)$. The critical values of $J / t$ record the parameter values where a level crossing occurs within the interval $0.0 \leq J / t<1.0$.

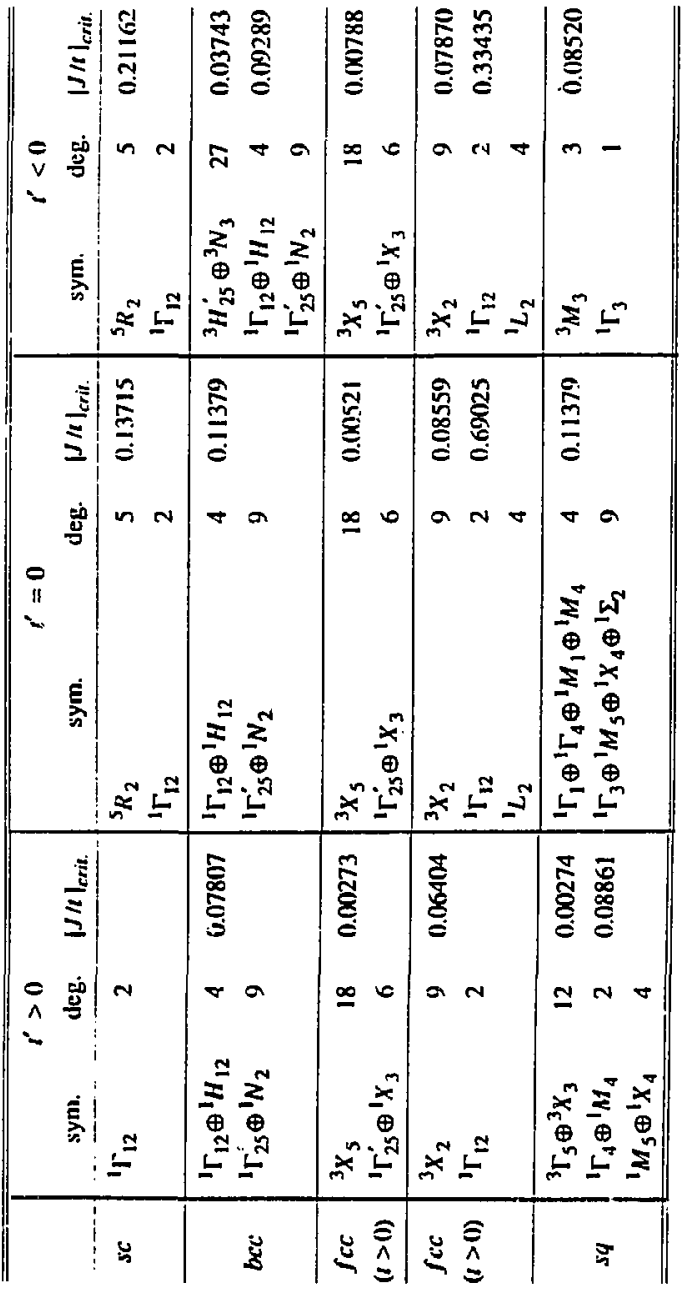




\begin{tabular}{|c|c|c|c|c|c|c|c|c|c|}
\hline & \multicolumn{3}{|c|}{$t>0$} & \multicolumn{3}{|l|}{$t^{\prime}=0$} & \multicolumn{3}{|l|}{$i<0$} \\
\hline & sym. & deg. & $|J / /|_{\text {cris. }}$ & sym. & deg. & $|J / t|_{c r i t .}$ & sym. & deg. & {$[\mathrm{J} / \mathrm{l}]_{\text {eris. }}$} \\
\hline$s c$ & $\begin{array}{l}{ }^{4} R_{2} \\
{ }^{2} X_{2}\end{array}$ & $\begin{array}{l}4 \\
6\end{array}$ & 0.22719 & $\begin{array}{l}{ }^{4} R_{2} \\
{ }^{2} X_{2}\end{array}$ & $\begin{array}{l}4 \\
6\end{array}$ & 0.28014 & $\begin{array}{l}{ }^{4} R_{2} \\
{ }^{2} X_{2}\end{array}$ & $\begin{array}{l}4 \\
6\end{array}$ & $\overline{0.30507}$ \\
\hline$b c c$ & $\begin{array}{l}{ }^{2} N_{1} \oplus^{2} N_{4} \\
{ }^{2} \Gamma_{15}^{\prime} \oplus^{2} H_{15} \\
\oplus^{2} N_{2} \oplus^{2} N_{3}\end{array}$ & $\begin{array}{l}24 \\
36\end{array}$ & 0.13027 & $\begin{array}{l}{ }^{2} N_{1} \oplus{ }^{2} N_{4} \\
{ }^{2} \Gamma_{15}^{\circ} \oplus^{2} H_{15} \oplus^{2} N_{2} \oplus^{2} N_{3}\end{array}$ & $\begin{array}{l}24 \\
36\end{array}$ & 0.21214 & $\begin{array}{l}{ }^{2} N_{1} \oplus{ }^{2} N_{4} \\
{ }^{2} \Gamma_{15}^{\prime} \oplus^{2} H_{15} \oplus^{2} N_{2} \oplus{ }^{2} N_{3} \\
{ }^{2} \Gamma_{25}^{\prime} \oplus \oplus^{2} N_{2}\end{array}$ & $\begin{array}{l}24 \\
36 \\
18\end{array}$ & $\begin{array}{l}0.20073 \\
0.82705\end{array}$ \\
\hline $\begin{array}{c}f c c \\
(1>0)\end{array}$ & $\begin{array}{l}{ }^{4} L_{2} \\
{ }^{2} L_{3}\end{array}$ & $\begin{array}{l}16 \\
16\end{array}$ & 0.00216 & $\begin{array}{l}{ }^{4} L_{2} \\
{ }^{2} L_{3}\end{array}$ & $\begin{array}{l}16 \\
16\end{array}$ & 0.00432 & $\begin{array}{l}{ }^{4} L_{2} \\
{ }^{2} L_{3}\end{array}$ & $\begin{array}{l}16 \\
16\end{array}$ & 0.00690 \\
\hline $\begin{array}{c}f c c \\
(\ell<0)\end{array}$ & $\begin{array}{l}{ }^{2} X_{1} \\
{ }^{2} L_{3} \\
{ }^{2} X_{5}\end{array}$ & $\begin{array}{r}6 \\
16 \\
12\end{array}$ & $\begin{array}{l}0.65744 \\
0.85651\end{array}$ & $\begin{array}{l}{ }^{2} X_{1} \\
{ }^{2} X_{5}\end{array}$ & $\begin{array}{r}6 \\
12\end{array}$ & 0.52150 & $\begin{array}{l}2 x_{1} \\
2 x_{5}\end{array}$ & $\begin{array}{r}6 \\
12\end{array}$ & 0.30260 \\
\hline$s y$ & $\begin{array}{l}{ }^{2} \Sigma_{1} \\
{ }^{2} \Sigma_{2}\end{array}$ & $\begin{array}{l}\text { R } \\
8\end{array}$ & 0.16958 & $\begin{array}{l}{ }^{2} X_{1} \oplus^{2} X_{2} \oplus^{2} \Sigma_{1} \oplus^{2} \Sigma_{2} \\
{ }^{2} \Gamma_{2} \oplus^{2} \Gamma_{5} \oplus^{2} M_{2} \oplus^{2} M_{5} \\
\oplus^{2} X_{3} \oplus^{2} X_{4} \oplus 2^{2} \Sigma_{2}\end{array}$ & $\begin{array}{l}24 \\
36 \\
\end{array}$ & 0.21214 & $\begin{array}{l}{ }^{2} x_{1} \\
{ }^{2} \Sigma_{2}\end{array}$ & $\begin{array}{l}4 \\
8\end{array}$ & 0.27940 \\
\hline
\end{tabular}




\begin{tabular}{|c|c|c|c|c|c|c|c|c|c|}
\hline & \multicolumn{3}{|c|}{$r^{\prime}>0$} & \multicolumn{3}{|c|}{$r^{\prime}=0$} & \multicolumn{3}{|c|}{$t<0$} \\
\hline & sym. & deg. & $|J / \||_{\text {crit. }}$ & sym. & deg. & $|S / \||_{\text {crid. }}$ & sym. & deg. & {$[S / l]_{\text {crit. }}$} \\
\hline sc & ${ }^{1} \Gamma_{12}$ & 2 & & $\begin{array}{l}{ }^{2} \Gamma_{1} \\
{ }^{1} \Gamma_{12} \\
\end{array}$ & $\begin{array}{l}1 \\
2\end{array}$ & 0.04489 & $\begin{array}{l}{ }^{1} \Gamma_{1} \\
{ }^{1} \Gamma_{12} \\
\end{array}$ & $\begin{array}{l}1 \\
2 \\
-1\end{array}$ & 0.21765 \\
\hline$b c c$ & $\begin{array}{l}{ }^{1} \Gamma_{1} \oplus^{1} \Gamma_{12} \oplus^{1} H_{2} \\
{ }^{1} \Gamma_{2}^{\prime} \oplus^{1} N_{2}\end{array}$ & $\begin{array}{l}4 \\
9\end{array}$ & $0.13 \mathrm{M} \times 7$ & $\begin{array}{l}{ }^{1} \Gamma_{1} \oplus{ }^{1} \Gamma_{12} \oplus^{1} H_{2} \\
{ }^{1} \Gamma_{25}^{\prime} \oplus^{1} N_{2}\end{array}$ & $\begin{array}{l}4 \\
9\end{array}$ & 0.21355 & $\begin{array}{l}{ }^{1} \Gamma_{1} \oplus^{1} \Gamma_{12} \oplus{ }^{\prime} H_{2} \\
{ }^{1} \Gamma_{25}^{\prime} \oplus^{1} N_{2} \\
{ }^{1} \Gamma_{1}\end{array}$ & $\begin{array}{l}4 \\
9 \\
1\end{array}$ & $\begin{array}{l}0.20287 \\
0.54656\end{array}$ \\
\hline $\begin{array}{c}f c c \\
(t>0)\end{array}$ & ${ }^{1} \Gamma_{12}$ & 2 & & ${ }^{1} \Gamma_{12}$ & 2 & & ${ }^{1} \Gamma_{12}$ & 2 & \\
\hline $\begin{array}{l}f c c \\
(l<0)\end{array}$ & $\begin{array}{l}{ }^{1} \Gamma_{1} \\
{ }^{t} \Gamma_{25}^{\prime} \oplus{ }^{i} x_{3}\end{array}$ & $\begin{array}{l}1 \\
6\end{array}$ & 0.63022 & $\begin{array}{l}{ }^{1} \Gamma_{1} \\
{ }^{\prime} \Gamma_{2}^{\prime} x^{\prime} x_{3} \\
{ }^{1} L_{2}\end{array}$ & $\begin{array}{l}1 \\
6 \\
4\end{array}$ & $\begin{array}{l}0.49013 \\
0.80484\end{array}$ & $\begin{array}{l}{ }^{1} \Gamma_{1} \\
{ }^{1} \Gamma_{25}^{\prime} \oplus^{\prime} X_{3} \\
{ }^{1} L_{2} \\
{ }^{1} \Gamma_{1} \oplus^{1} \Gamma_{12}\end{array}$ & $\begin{array}{l}1 \\
6 \\
4 \\
3\end{array}$ & $\begin{array}{l}0.30941 \\
0.42641 \\
0.78826\end{array}$ \\
\hline$s q$ & $\begin{array}{l}{ }^{1} \Gamma_{1} \\
{ }^{1} \Gamma_{3}\end{array}$ & $\begin{array}{l}1 \\
1\end{array}$ & 0.17094 & $\begin{array}{l}{ }^{1} \Gamma_{1} \oplus^{1} \Gamma_{4} \oplus^{1} M_{4} \\
{ }^{1} \Gamma_{3} \oplus^{1} M_{5} \oplus^{1} X_{4} \oplus^{1} \Sigma_{2}\end{array}$ & $\begin{array}{l}4 \\
9 \\
\end{array}$ & 0.21355 & $\begin{array}{l}{ }^{1} \Gamma_{1} \\
{ }^{1} M_{5} \oplus{ }^{1} X_{4}\end{array}$ & $\begin{array}{l}1 \\
4\end{array}$ & 0.28349 \\
\hline
\end{tabular}




\begin{tabular}{|c|c|c|c|c|c|c|c|c|c|}
\hline \multirow{2}{*}{ - } & \multicolumn{3}{|c|}{$r>0$} & \multicolumn{3}{|l|}{$r=0$} & \multicolumn{3}{|c|}{$r^{\prime}<0$} \\
\hline & sym. & deg. & {$\left[J \|\left.\right|_{\text {erit. }}\right.$} & sym. & deg. & {$[J / t]_{\text {crit. }}$} & sym. & deg. & $\mid \boldsymbol{J} \boldsymbol{\|} \boldsymbol{|}_{\text {cris. }}$ \\
\hline$s c$ & ${ }^{2} X_{1}$ & 6 & & $\begin{array}{l}{ }^{8} R_{1} \\
{ }^{2} x_{1}\end{array}$ & $\begin{array}{l}8 \\
6\end{array}$ & 0.05878 & $\begin{array}{l}{ }^{8} R_{1} \\
{ }^{6} \Gamma_{1} \\
{ }^{4} R_{1} \\
{ }^{2} X_{1} \\
{ }^{2} \Gamma_{1}\end{array}$ & $\begin{array}{l}8 \\
6 \\
4 \\
6 \\
2\end{array}$ & $\begin{array}{l}0.11763 \\
0.14158 \\
0.27134 \\
0.34310\end{array}$ \\
\hline$b c c$ & $\begin{array}{l}{ }^{6} N_{1} \\
{ }^{4} \Gamma_{12} \oplus^{4} H_{12} \\
{ }^{2} N_{1}\end{array}$ & $\begin{array}{l}36 \\
16 \\
12\end{array}$ & $\begin{array}{l}0.02488 \\
0.09174\end{array}$ & $\begin{array}{l}{ }^{8} H_{1} \\
{ }^{6} N_{1} \\
{ }^{4} \Gamma_{12} \oplus^{4} H_{12} \\
{ }^{2} N_{1}\end{array}$ & $\begin{array}{l}8 \\
36 \\
16 \\
12\end{array}$ & $\begin{array}{l}0.10383 \\
0.11856 \\
0.16573\end{array}$ & $\begin{array}{l}{ }^{8} H_{1} \\
{ }^{6} \Gamma_{1} \\
{ }^{4} H_{1} \\
{ }^{2} \Gamma_{1}\end{array}$ & $\begin{array}{l}8 \\
6 \\
4 \\
2\end{array}$ & $\begin{array}{l}0.14286 \\
0.20000 \\
0.33333\end{array}$ \\
\hline $\begin{array}{c}f c c \\
(t>0)\end{array}$ & ${ }^{2} \Gamma_{2} \oplus^{2} X_{1} \oplus^{2} X_{2}$ & 14 & & ${ }^{2} \Gamma_{2} \oplus^{2} X_{1} \oplus^{2} X_{2}$ & 14 & & ${ }^{2} \Gamma_{2} \oplus^{2} X_{1} \oplus^{2} X_{2}$ & 14 & \\
\hline $\begin{array}{c}f c c \\
(t<0)\end{array}$ & $\begin{array}{l}{ }^{8} \Gamma_{1} \\
{ }^{6} L_{1} \\
{ }^{4} \Gamma_{25}^{0} \oplus^{4} X_{3} \\
{ }^{2} L_{1} \\
{ }^{2} X_{5} \\
{ }^{2} L_{2} \oplus^{2} L_{3} \\
{ }^{2} X_{1} \oplus^{2} X_{2}\end{array}$ & $\begin{array}{r}8 \\
24 \\
24 \\
8 \\
12 \\
24 \\
12\end{array}$ & $\begin{array}{l}0.08785 \\
0.11555 \\
0.22932 \\
0.26969 \\
0.34658 \\
0.69048\end{array}$ & $\begin{array}{l}{ }^{8} \Gamma_{1} \\
{ }^{6} L_{3} \\
{ }^{4} \Gamma_{25}^{\prime} \oplus{ }^{4} X_{3} \\
{ }^{4} L_{3} \\
{ }^{4} \Gamma_{1} \oplus \oplus^{4} \Gamma_{12} \oplus{ }^{4} X_{1} \\
{ }^{2} X_{1} \oplus^{2} X_{2}\end{array}$ & $\begin{array}{r}8 \\
24 \\
24 \\
32 \\
24 \\
12\end{array}$ & $\begin{array}{l}0.12633 \\
0.17794 \\
0.20209 \\
0.25(000) \\
0.33333\end{array}$ & $\begin{array}{l}{ }^{8} \Gamma_{1} \\
{ }^{6} x_{1} \\
{ }^{4} \Gamma_{1} \oplus^{4} \Gamma_{12} \oplus^{4} x_{1} \\
{ }^{2} x_{1} \oplus^{2} x_{2}\end{array}$ & $\begin{array}{l}8 \\
18 \\
24 \\
12\end{array}$ & $\begin{array}{l}0.14286 \\
0.20000 \\
0.33333\end{array}$ \\
\hline$s q$ & $\begin{array}{l}{ }^{8} M_{1} \\
{ }^{6} X_{1} \\
{ }^{4} \Gamma_{1} \oplus{ }^{4} M_{1} \\
{ }^{2} X_{1}\end{array}$ & $\begin{array}{r}8 \\
12 \\
8 \\
4\end{array}$ & $\begin{array}{l}0.03336 \\
0.05991 \\
0.12436\end{array}$ & $\begin{array}{l}{ }^{2} M_{1} \\
{ }^{6} X_{1} \oplus^{6} \Sigma_{1} \\
{ }^{4} \Gamma_{1} \oplus^{4} \Gamma_{4} \oplus^{4} M_{1} \oplus^{4} M_{4} \\
{ }^{2} X_{1} \oplus^{2} \Sigma_{1}\end{array}$ & $\begin{array}{r}8 \\
36 \\
16 \\
12\end{array}$ & $\begin{array}{l}0.10383 \\
0.11856 \\
0.16573\end{array}$ & $\begin{array}{l}M_{i} \\
{ }^{4} \Gamma_{4} \oplus^{4} M_{4} \\
{ }^{2} \Sigma_{1} \\
{ }^{2} \Gamma_{1}\end{array}$ & $\begin{array}{l}8 \\
8 \\
8 \\
2\end{array}$ & $\begin{array}{l}0.13165 \\
0.19593 \\
0.53125\end{array}$ \\
\hline
\end{tabular}


Table 3.9. Accidental degeneracies in the $t-t^{0}-\jmath$ model at $J=0$.

\begin{tabular}{c|c|lr}
\hline \hline latice & $N$ & \multicolumn{1}{|c}{ ground state } & deg. \\
\hline$f c c$ & 2 & ${ }^{3} X_{2} \oplus^{1} \Gamma_{12}$ & 11 \\
$(t<0)$ & & & \\
$b c c$ & 4 & ${ }^{3} H_{25}^{\prime} \oplus^{3} N_{3} \oplus^{1} \Gamma_{12} \oplus^{1} H_{12}$ & 31 \\
$t=0$ & & & \\
$s q$ & & ${ }^{3} \Gamma_{5} \oplus^{3} M_{3} \oplus^{3} X_{3} \oplus^{3} \Sigma_{2}$ & \\
$t=0$ & 4 & ${ }^{1} \Gamma_{1} \oplus^{1} \Gamma_{4} \oplus^{1} M_{1} \oplus^{1} M_{4}$ & 31 \\
$f c c$ & 6 & ${ }^{5} \Gamma_{2} \oplus^{3} X_{2} \oplus^{1} \Gamma_{12}$ & 16 \\
$(t>0)$ & & & \\
$f c c$ & & ${ }^{6} X_{2} \oplus^{4} L_{2} \oplus^{4} \Gamma_{12} \oplus^{4} X_{1} \oplus^{4} X_{2}$ & \\
$(t>0)$ & 7 & $\oplus^{2} L_{3} \oplus^{2} \Gamma_{2} \oplus^{2} X_{1} \oplus^{2} X_{2}$ & 96 \\
$a l l$ & 8 & $a l l$ & 256 \\
\hline \hline
\end{tabular}


Table 3.10. Maximum number of many-body states lying within $0.1|t|$ of the ground-state energy (including the degeneracy of the ground-state manifold). Potential HF are highlighted in bold.

\begin{tabular}{|c|c|c|c|c|c|c|c|c|c|c|c|c|c|c|c|}
\hline$N$ & & $s c$ & & & $b c c$ & & & $(t>$ & & & $:(t$ & & & $s q$ & \\
\hline$i^{\prime}$ & + & 0 & . & + & 0 & - & + & 0 & - & + & 0 & - & + & 0 & - \\
\hline 1 & & 2 & & & 2 & & & 2 & & & 6 & & & 2 & \\
\hline 2 & & 1 & & & 1 & & & 1 & & & 11 & & & 1 & \\
\hline 3 & 4 & 4 & 18 & & 12 & & & 8 & & & 10 & & 8 & 12 & 4 \\
\hline 4 & 11 & 16 & 7 & & 40 & & & 24 & & & 11 & & 18 & 40 & 4 \\
\hline 5 & & 10 & & & 60 & & & 32 & & 28 & 34 & 34 & 16 & 60 & 12 \\
\hline 6 & 2 & 3 & 3 & & 13 & & & 16 & & 7 & 10 & 10 & 2 & 13 & 5 \\
\hline 7 & 6 & 38 & 18 & 52 & 60 & 14 & & 96 & & 48 & 98 & 42 & 28 & 76 & 54 \\
\hline 8 & & 256 & & & 256 & & & 256 & & & 256 & & 256 & 256 & 256 \\
\hline
\end{tabular}


Table 3.11. Maximum number of noninteracting-electron states lying within $0.1 / t \mid$ of the ground-state energy (including the degeneracy of the ground-state manifold).

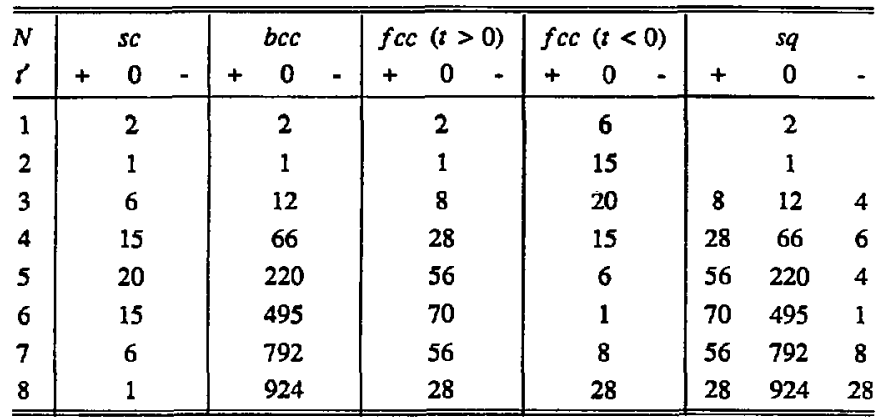


Table 3.12. Many-body solutions to the $t-t^{\prime}-J$ model that exhibit strong HF character. The range of interaction strength $J / t$ where the solutions are HF and the total spin of the ground state $S_{G S}$ are included.

\begin{tabular}{ccccc}
\hline \hline & lattice & $t$ & $J / t$ & $s_{G S}$ \\
\hline 3 & $s c$ & $t<0$ & $0.0<J / t<0.05$ & $1 / 2$ \\
4 & $s c$ & $t=0$ & $0.0<J / t<0.04$ & 2 or 0 \\
7 & $s c$ & $t=0$ & $0.05<J / t<0.065$ & $7 / 2$ or $1 / 2$ \\
7 & $s c$ & $t<0$ & $0.12<J / t<0.13$ & $7 / 2$ or $5 / 2$ \\
7 & $f c c(t>0)$ & all $t$ & $0.0<J / t<0.01$ & $1 / 2$ \\
7 & $f c c(t<0)$ & $t>0$ & $0.1<J / t<0.12$ & $5 / 2$ or $3 / 2$ \\
7 & $f c c(t<0)$ & $t=0$ & $0.17<J / t<0.19$ & $5 / 2$ or $3 / 2$ \\
7 & $s q$ & $t<0$ & $0.15<J / t<0.16$ & $3 / 2$ \\
\hline \hline
\end{tabular}


Table 3.13. Character table for the space group of the eight-site sc cluster. The space group is isomorphic to the cubic point group $O_{h}$, with an origin at the center of the small cluster, when spherically syminetric orbitals are placed at the lartice sites. $E$ is the identity, $C_{n}^{m}$ is the rotation of $2 \pi \mathrm{m} / n$ about an $n$-fold axis, and $J$ is the inversion. Both the space group and the point group notations for the irreducible representations are included.

\begin{tabular}{|c|c|c|c|c|c|c|c|c|c|c|c|}
\hline & & $E$ & $\begin{array}{r}3 \\
C_{4}^{2}\end{array}$ & $\begin{array}{c}6 \\
C_{4}\end{array}$ & $\begin{array}{r}6 \\
C_{2}\end{array}$ & $\begin{array}{c}8 \\
C_{3}\end{array}$ & $\begin{array}{l}1 \\
j\end{array}$ & $\begin{array}{c}3 \\
J C_{4}^{2}\end{array}$ & $\begin{array}{r}6 \\
J C_{4}\end{array}$ & $\begin{array}{r}6 \\
J C_{2}\end{array}$ & $\begin{array}{r}8 \\
J C_{3}\end{array}$ \\
\hline$\Gamma_{\mathrm{I}}$ & $A_{\mathrm{Ig}}$ & 1 & 1 & 1 & 1 & 1 & 1 & 1 & 1 & 1 & 1 \\
\hline$\Gamma_{2}$ & $A_{28}$ & 1 & 1 & -1 & -1 & 1 & 1 & 1 & -1 & -1 & 1 \\
\hline$\Gamma_{12}$ & $E_{g}$ & 2 & 2 & 0 & 0 & -1 & 2 & 2 & 0 & 0 & -1 \\
\hline$M_{2}$ & $T_{1 g}$ & 3 & -1 & 1 & -1 & 0 & 3 & -1 & 1 & -1 & 0 \\
\hline$M_{1}$ & $T_{2 g}$ & 3 & -1 & -1 & 1 & 0 & 3 & -1 & -1 & 1 & 0 \\
\hline$R_{2}$ & $A_{1 \mu}$ & 1 & 1 & 1 & 1 & 1 & -1 & -1 & -1 & -1 & -1 \\
\hline$R_{1}$ & $A_{2 u}$ & 1 & 1 & -1 & -1 & 1 & -1 & -1 & 1 & 1 & -1 \\
\hline$R_{12}$ & $E_{u}$ & 2 & 2 & 0 & 0 & -1 & -2 & -2 & 0 & 0 & I \\
\hline$x_{1}$ & $T_{14}$ & 3 & -1 & 1 & -1 & 0 & -3 & 1 & -1 & 1 & 0 \\
\hline$X_{2}$ & $T_{2 u}$ & 3 & -1 & -1 & 1 & 0 & -3 & 1 & 1 & -1 & 0 \\
\hline
\end{tabular}


Table 3.14. Character table for the space group of the eight-site $b c c$ cluster. The space group operations are constructed by a point group operation with origin at site 1 followed by a translation. The symbol 0 denotes no translation, $\tau$ denotes a nearestneighbor translation, and $\theta$ is a next-nearest-neighbor translation. The subscripts $\|, 1$, and $\angle$ refer to translations that are parallel to, perpendicular to, and at an angle to the rotation axis of the point group operation.

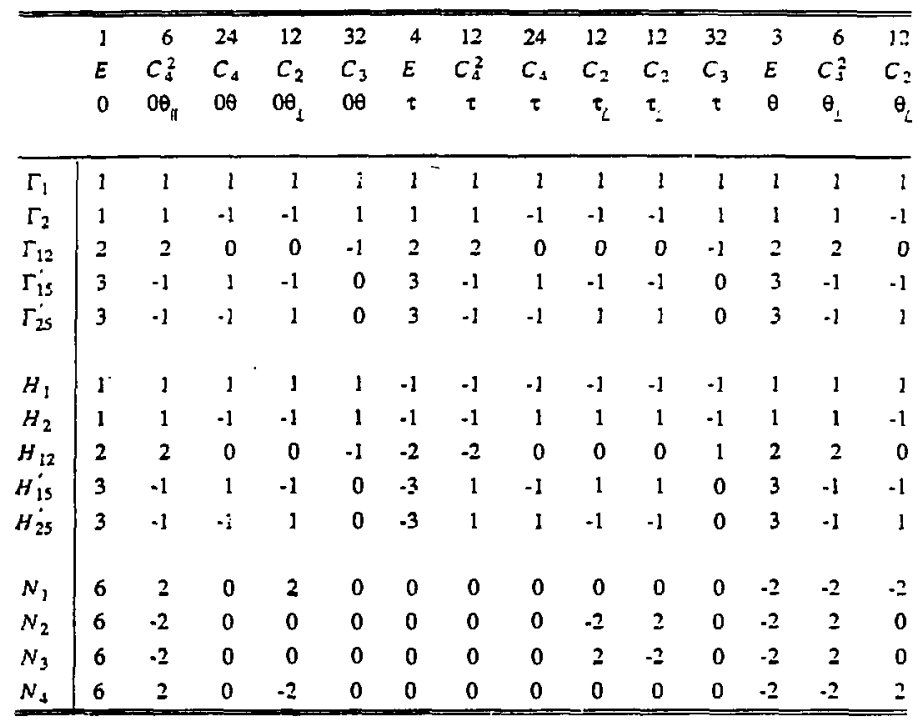


Table 3.15. Character table for the space group of the eight-site $f c c$ cluster. The notation is identical to that of Table 3.14.

\begin{tabular}{lrrrrrrrrrrrrr}
\hline & 1 & 6 & 24 & 12 & 32 & 6 & 6 & 12 & 24 & 24 & 1 & 12 & 32 \\
& $E$ & $C_{4}^{2}$ & $C_{4}$ & $C_{2}$ & $C_{3}$ & $E$ & $C_{4}^{2}$ & $C_{4}^{2}$ & $C_{4}$ & $C_{2}$ & $E$ & $C_{2}$ & $C_{3}$ \\
& 0 & $0 \theta$ & $0 \theta \tau_{i}$ & $0 \tau_{11}$ & $0 \tau_{1}$ & $\tau$ & $\tau_{1}$ & $\tau_{L}$ & $\tau_{L}$ & $\tau_{L}$ & 2 & $\theta \tau_{L}$ & $\theta \tau_{L}$ \\
\hline$\Gamma_{1}$ & 1 & 1 & 1 & 1 & 1 & 1 & 1 & 1 & 1 & 1 & 1 & 1 & 1 \\
$\Gamma_{2}$ & 1 & 1 & -1 & -1 & 1 & 1 & 1 & 1 & -1 & -1 & 1 & -1 & 1 \\
$\Gamma_{12}$ & 2 & 2 & 0 & 0 & -1 & 2 & 2 & 2 & 0 & 0 & 2 & 0 & -1 \\
$\Gamma_{15}^{\prime}$ & 3 & -1 & 1 & -1 & 0 & 3 & -1 & -1 & 1 & -1 & 3 & -1 & 0 \\
$\Gamma_{35}^{\prime}$ & 3 & -1 & -1 & 1 & 0 & 3 & -1 & -1 & -1 & 1 & 3 & 1 & 0 \\
& & & & & & & & & & & & & \\
$X_{1}$ & 3 & 3 & 1 & 1 & 0 & -1 & -1 & -1 & -1 & -1 & 3 & 1 & 0 \\
$X_{2}$ & 3 & 3 & -1 & -1 & 0 & -1 & -1 & -1 & 1 & 1 & 3 & -1 & 0 \\
$X_{3}$ & 3 & -1 & -1 & 1 & 0 & -1 & 3 & -1 & 1 & -1 & 3 & 1 & 0 \\
$X_{4}$ & 3 & -1 & 1 & -1 & 0 & -1 & 3 & -1 & -1 & 1 & 3 & -1 & 0 \\
$X_{5}$ & 6 & -2 & 0 & 0 & 0 & -2 & -2 & 2 & 0 & 0 & 6 & 0 & 0 \\
& & & & & & & & & & & & & \\
$L_{1}$ & 4 & 0 & 0 & 2 & 1 & 0 & 0 & 0 & 0 & 0 & -4 & -2 & -1 \\
$L_{2}$ & 4 & 0 & 0 & -2 & 1 & 0 & 0 & 0 & 0 & 0 & -4 & 2 & -1 \\
$L_{3}$ & 8 & 0 & 0 & 0 & -1 & 0 & 0 & 0 & 0 & 0 & -8 & 0 & 1 \\
\hline \hline
\end{tabular}


Table 3.16. Character table for the space group of the eight-site $s q$ cluster. The symbol $\sigma$ denotes the mirror planes perpendicular to the $x$ - and $y$-axes and $\sigma$ denotes the mirror planes perpendicular to the diagonals $x \pm y$. The translations are denoted by 0 (no translation), $\tau$ (nearest-neighbor translation), $\theta$ (next-nearest-neighbor), and $\Omega$ (third-nearest-neighbor). The subscripts II and $\perp$ refer to translations parallel to or perpendicular to the normals of the mirror planes.

\begin{tabular}{|c|c|c|c|c|c|c|c|c|c|c|c|c|c|c|c|c|}
\hline & $\begin{array}{l}1 \\
E \\
0\end{array}$ & $\begin{array}{c}8 \\
c_{4}\end{array}$ & $\begin{array}{c}2 \\
c_{4}^{2} \\
0 \Omega\end{array}$ & $\begin{array}{c}4 \\
\sigma \\
0 \Omega\end{array}$ & $\begin{array}{c}4 \\
\sigma^{\prime} \\
c \theta_{1}\end{array}$ & $\begin{array}{l}4 \\
E \\
\tau\end{array}$ & $C_{4}^{8}$ & $\begin{array}{c}4 \\
C_{1}^{2} \\
\tau\end{array}$ & $\begin{array}{l}4 \\
\sigma \\
t_{H}\end{array}$ & $\begin{array}{l}4 \\
0 \\
\tau_{1}\end{array}$ & $\begin{array}{l}8 \\
\sigma^{\prime} \\
5\end{array}$ & $\begin{array}{l}2 \\
E \\
\theta\end{array}$ & $\begin{array}{c}2 \\
C_{4}^{2} \\
\theta\end{array}$ & $\begin{array}{l}4 \\
0 \\
\theta\end{array}$ & $\begin{array}{c}4 \\
\sigma^{\prime} \\
\theta_{11} \Omega\end{array}$ & $\begin{array}{l}1 \\
E \\
\Omega\end{array}$ \\
\hline$\Gamma_{1}$ & 1 & 1 & 1 & 1 & 1 & 1 & 1 & 1 & 1 & 1 & 1 & 1 & 1 & 1 & 1 & 1 \\
\hline$\Gamma_{2}$ & 1 & 1 & 1 & -1 & -1 & 1 & 1 & 1 & -1 & -1 & -1 & 1 & 1 & -1 & -1 & 1 \\
\hline$\Gamma_{3}$ & 1 & -1 & 1 & 1 & -1 & 1 & -1 & 1 & 1 & 1 & -1 & 1 & 1 & I & $-i$ & 1 \\
\hline$\Gamma_{4}$ & 1 & -1 & 1 & -1 & 1 & 1 & -1 & 1 & -1 & -1 & 1 & 1 & $\mathbf{l}$ & -1 & 1 & 1 \\
\hline$\Gamma_{s}$ & 2 & 0 & -2 & 0 & 0 & 2 & 0 & $\begin{array}{l}-2 \\
\end{array}$ & 0 & 0 & 0 & 2 & -2 & 0 & 0 & 2 \\
\hline$M_{l}$ & 1 & 1 & 1 & 1 & 1 & -1 & -1 & -1 & -1 & -1 & -1 & $\mathbf{J}$ & 1 & 1 & 1 & 1 \\
\hline$M_{2}$ & 1 & 1 & 1 & -1 & -1 & -1 & -1 & -1 & 1 & 1 & 1 & 1 & 1 & -1 & -1 & 1 \\
\hline$M_{3}$ & 1 & -1 & 1 & 1 & -1 & -1 & 1 & -1 & -1 & -1 & 1 & 1 & 1 & 1 & -1 & 1 \\
\hline$M_{4}$ & 1 & -1 & 1 & -1 & 1 & -1 & 1 & -1 & 1 & 1 & -1 & 1 & 1 & -1 & 1 & 1 \\
\hline$M_{9}$ & 2 & 0 & -2 & 0 & 0 & -2 & 0 & 2 & 0 & 0 & 0 & 2 & -2 & 0 & 0 & 2 \\
\hline$x_{1}$ & 2 & 0 & 2 & 2 & 0 & 0 & 0 & 0 & 0 & 0 & 0 & -2 & -2 & -2 & 0 & 2 \\
\hline$x_{2}$ & 2 & 0 & 2 & -2 & $n$ & 0 & 0 & 0 & 0 & 0 & 0 & -2 & -2 & 2 & 0 & 2 \\
\hline$x_{3}$ & 2 & 0 & -2 & 0 & 0 & 0 & 0 & 0 & -2 & 2 & 0 & -2 & 2 & 0 & 0 & 2 \\
\hline$x_{4}$ & 2 & 0 & -2 & 0 & 0 & 0 & 0 & 0 & 2 & -2 & 0 & -2 & 2 & 0 & 0 & 2 \\
\hline$\Sigma_{1}$ & 4 & 0 & 0 & 0 & 2 & 0 & 0 & 0 & 0 & 0 & 0 & 0 & 0 & 0 & -2 & -4 \\
\hline$\Sigma_{2}$ & 4 & 0 & 0 & 0 & -2 & 0 & 0 & 0 & 0 & 0 & 0 & 0 & 0 & 0 & 2 & -4 \\
\hline
\end{tabular}


Table 3.17. Equivalence classes of the eight small-cluster sites in the $s c, b c c, f c c$, and $s q$ infinite lattices.

\begin{tabular}{c|c|c|c|c}
\hline \hline Class & $s c$ & \multicolumn{1}{c}{$f c c$} & $s q$ \\
\hline 1 & $(2 i, 2 j, 2 k)$ & $(4 i, 4 j, 4 k)$ & $(2 i, 2 j, 2 k)$ & $(2 i, 2 j)$ \\
& & $(4 i+2,4 j+2,4 k+2)$ & $i+j+k=$ even & $i+j=\varepsilon v e n$ \\
2 & $(2 i+1,2 j, 2 k)$ & $(4 i+2,4 j, 4 k)$ & $(2 i, 2 j+1,2 k+1)$ & $(2 i+1,2 j)$ \\
& & $(4 i, 4 j+2,4 k+2)$ & $i+j+k=$ even & $i+j=$ even \\
3 & $(2 i+1,2 j+1,2 k)$ & $(4 i, 4 j, 4 k+2)$ & $(2 i+1,2 j, 2 k+1)$ & $(2 i+1,2 j+1)$ \\
& & $(4 i+2,4 j+2,4 k)$ & $i+j+k=$ odd & $i+j=$ odd \\
4 & $(2 i, 2 j+1,2 k)$ & $(4 i, 4 j+2,4 k)$ & $(2 i+1,2 j+1,2 k)$ & $(2 i, 2 j+1)$ \\
& & $(4 i+2,4 j, 4 k+2)$ & $i+j+k=$ odd & $i+j=$ even \\
5 & $(2 i+1,2 j, 2 k+1)$ & $(4 i+1,4 j+1,4 k+1)$ & $(2 i, 2 j, 2 k)$ & $(2 i, 2 j)$ \\
& & $(4 i+3,4 j+3,4 k+3)$ & $i+j+k=$ odd & $i+j=$ odd \\
6 & $(2 i, 2 j, 2 k+1)$ & $(4 i+3,4 j+1,4 k+1)$ & $(2 i, 2 j+1,2 k+1)$ & $(2 i+1,2 j)$ \\
& & $(4 i+1,4 j+3,4 k+3)$ & $i+j+k=$ odd & $i+j=$ odd \\
7 & $(2 i, 2 j+1,2 k+1)$ & $(4 i+1,4 j+1,4 k+3)$ & $(2 i+1,2 j, 2 k+1)$ & $(2 i+1,2 j+1)$ \\
& & $(4 i+3,4 j+3,4 k+1)$ & $i+j+k=$ even & $i+j=$ even \\
& & $(4 i+1,4 j+3,4 k+1)$ & $(2 i+1,2 j+1,2 k)$ & $(2 i, 2 j+1)$ \\
& & $(4 i+3,4 j+1,4 k+3)$ & $i+j+k=$ even & $i+j=$ odd \\
\hline \hline
\end{tabular}


Figure 3.1. Eight-site cluster with PBC for the sc lattice in (a) real and (b) reciprocal space. The nearest neighbors of the site 1 are two each of the sites 2, 4, and 6 as indicated in (a). The four symmetry stars in (b) are $\Gamma=(0,0,0) ; R=(1,1,1) \pi / a$; $M=(1,1,0) \pi / a ;$ and $X=(1,0,0) \pi / a$.
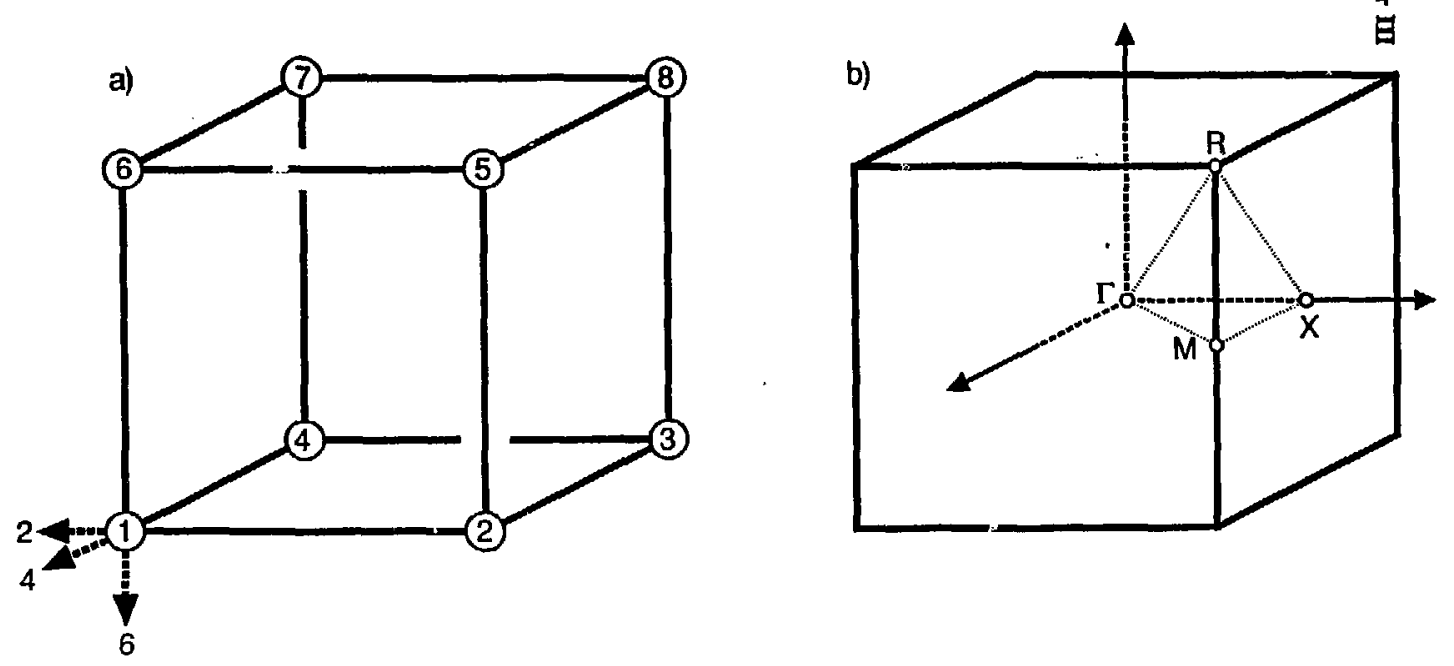
Figure 3.2. Eight-site cluster with PBC for the bcc lattice in (a) real and (b) reciprocal space. The dotted line in (a) is the bedy diagonal. The nearest neighbors of site 5 are two each of the sites $1,2,3$, and 4. The three symmetry stars in (b) are $\Gamma=(0,0,0) ; H=(2,0,0) \pi / a ;$ and $N=(1,1,0) \pi / a$.
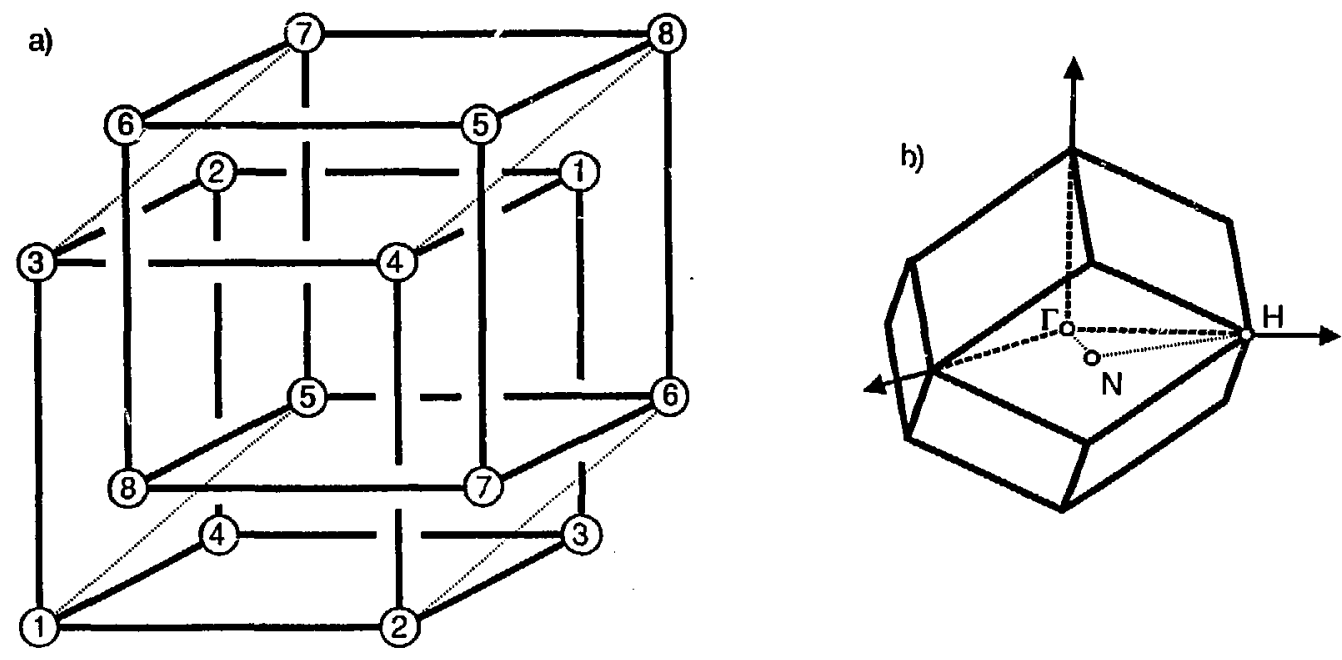
Figure 3.3. Eight-site cluster with PBC for the $f c c$ lattice in (a) real and (b) reciprocal space. The double-tetrahedral structure is highlighted with dotted lines in (a). The three symmetry stars ịn (b) are $\Gamma=(0,0,0) ; X=(0,0,2) \pi / a$; and $L=(1,1,1) \pi / a$.
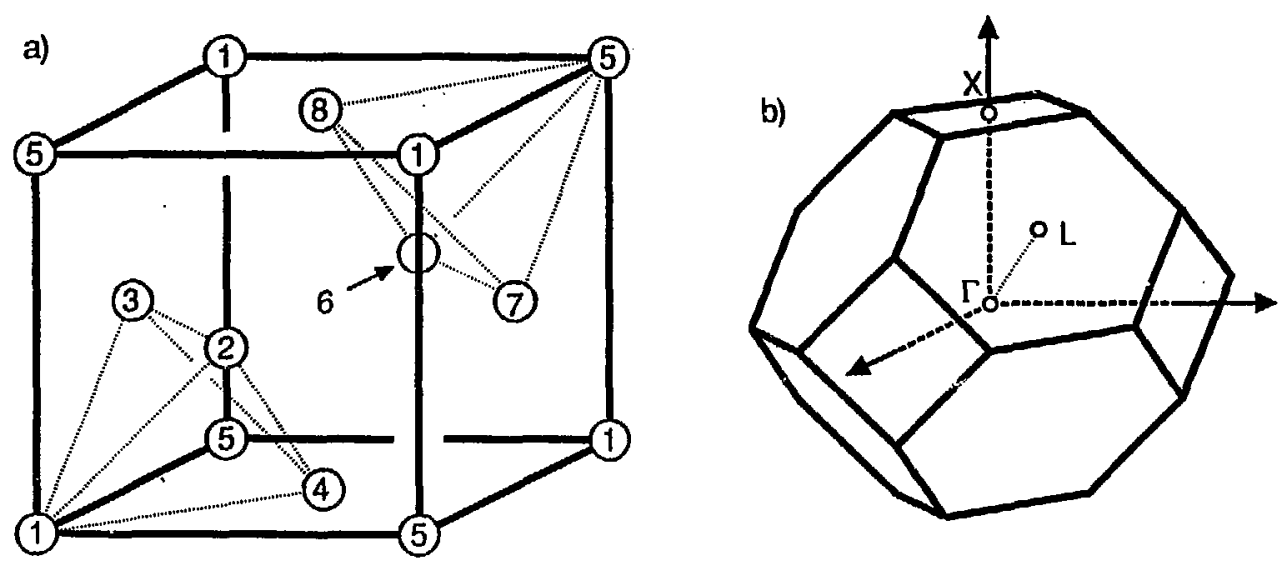
Figure 3.4. Eight-site cluster with PBC for the sq lattice in (a) real and (b) reciprocal space. The $2 \sqrt{2} a \times 2 \sqrt{2} a$ "primitive" cell is highlighted with dotted lines in (a). The four symmetry stars are $\Gamma=(0,0) ; M=(1,1) \pi / a ; X=(1,0) \pi / a$; and $\Sigma=(1,1) \pi / 2 a$.

a)

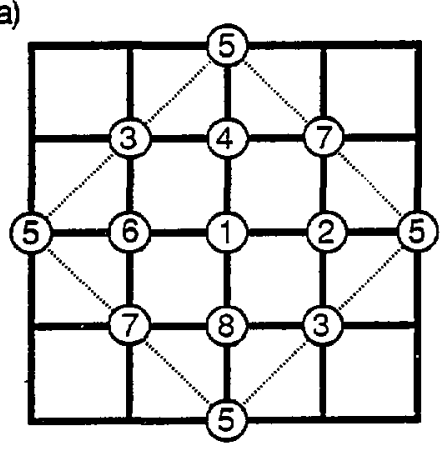

b)

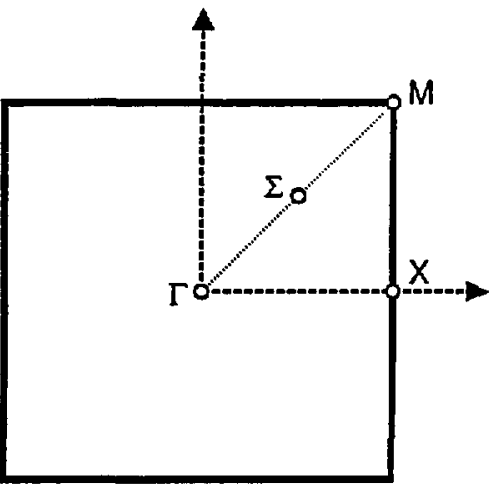


Figure 3.5. Stability phase diagram for the sc lattice. The horizontal axis is the interaction strength $J / t$ and the vertical axis is the electron filling $N$. Solid horizontal lines correspond to stable single-phases. Dotted vertical lines denote disproportiation instabilities or level crossings in the fixed- $N$ solutions. Level crossings are also marked by a black dot. Three cases have been calculated: (a) $t=0.15 \mid \mathrm{t}$ l; (b) $t^{\prime}=0.0$; and (c) $t^{\prime}=-0.15|t|$.

a)

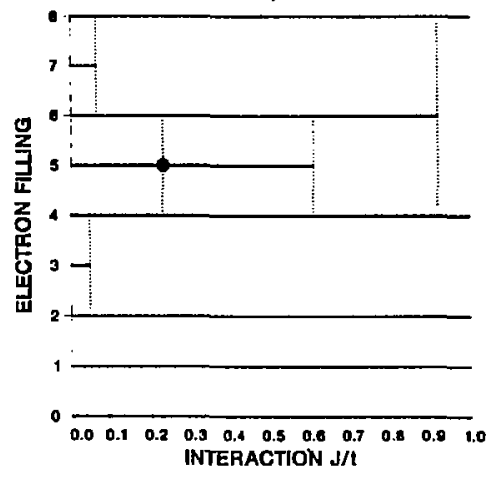

b)

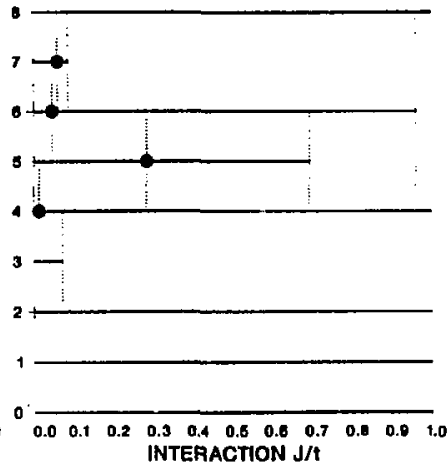

c)

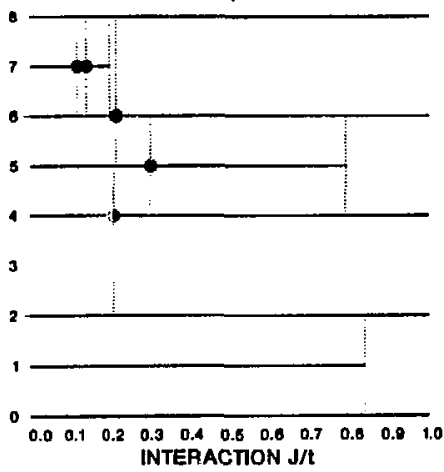


Figure 3.6. Stability phase diagram for the bcc lattice. Three cases have been calculated: (a) $t^{\prime}=0.5|t|$; (b) $t^{\prime}=0.0$; and (c) $t^{\prime}=-0.5|t|$. Note the phase islands for $N=4$.

a)

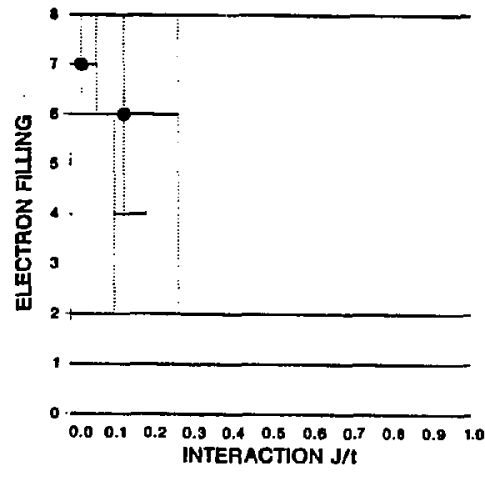

b)

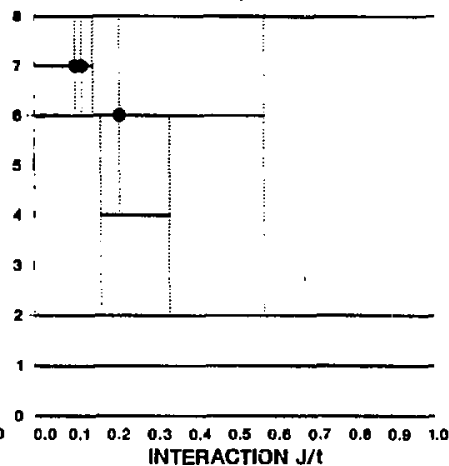

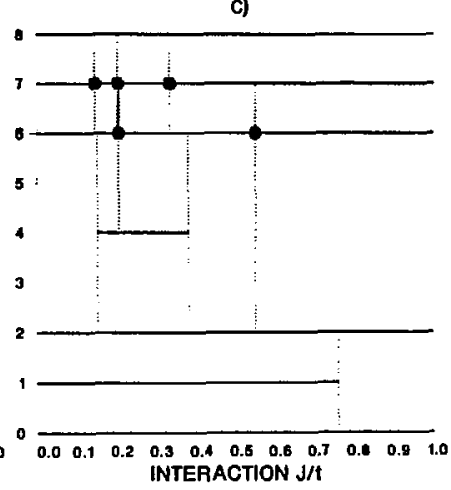


Figure 3.7. Stability phase diagram for the $f c c(t>0)$ lattice. Three cases have been calculated: (a) $t^{\prime}=0.15|t|$; (b) $t^{\prime}=0.0$; and (c) $t^{\prime}=-0.15|t|$.

a)

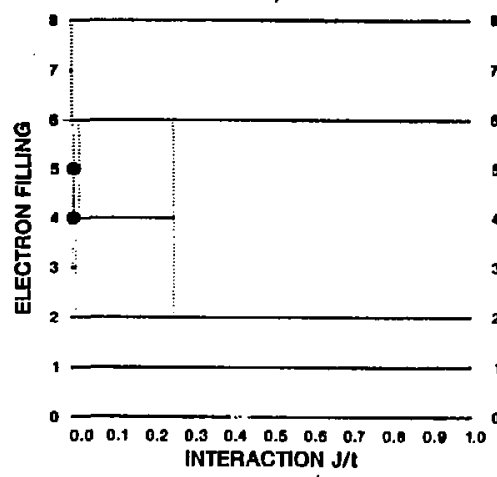

b)

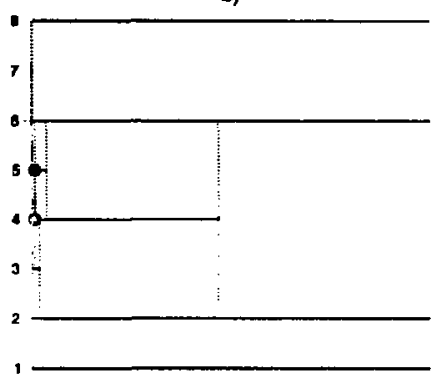

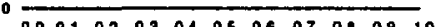
INTERACTION $\mathbf{J} / \mathrm{t}$

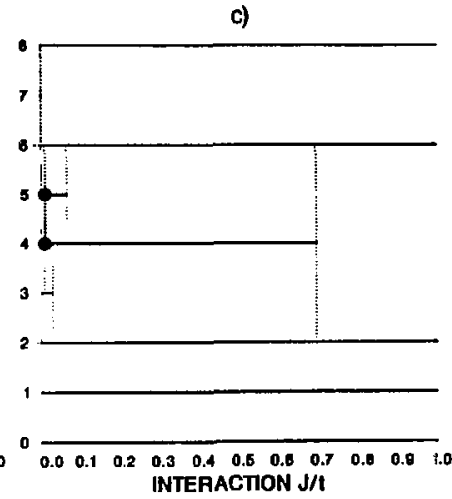


Figure 3.8. Stability phase diagram for the $f c c(t<0)$ latice. Three cases have been calculated: (a) $t^{\prime}=0.15|t|$; (b) $t^{\prime}=0.0$; and (c) $t^{\prime}=-0.151 t \mathrm{l}$. Note the phase islands for $N=4$ and $N=7$ and that $N=1$ is unstable.

a)

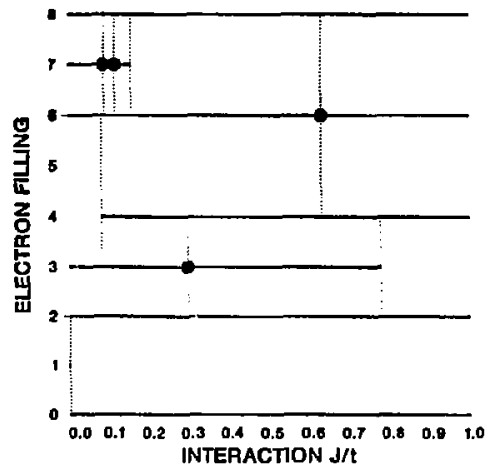

b)

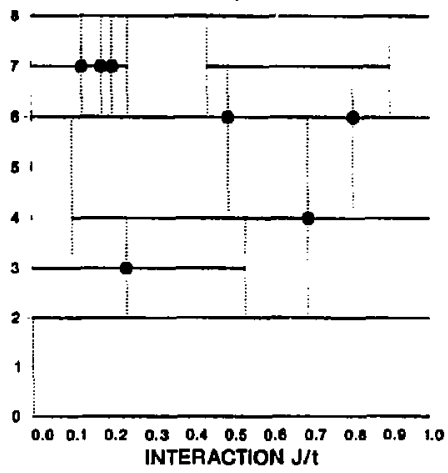

c)

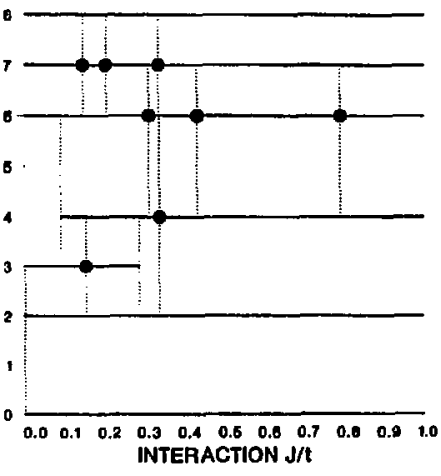


Figure 3.9. Stability phase diagram for the sq lattice. Three cases have been calculated: (a) $t^{\prime}=0.15|t|$; (b) $t=0.0$; and (c) $t^{\prime}=-0.15|t|$. Note the phase islands for $\boldsymbol{N}=\mathbf{4}$.

a)

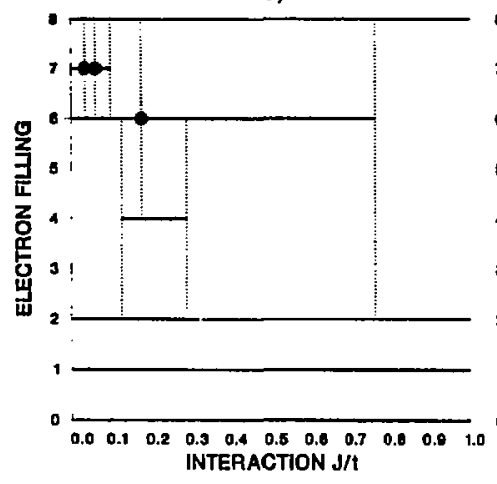

b)

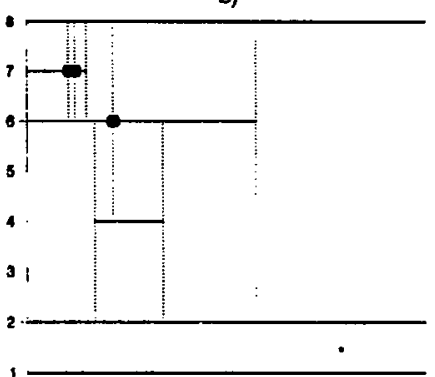

c)

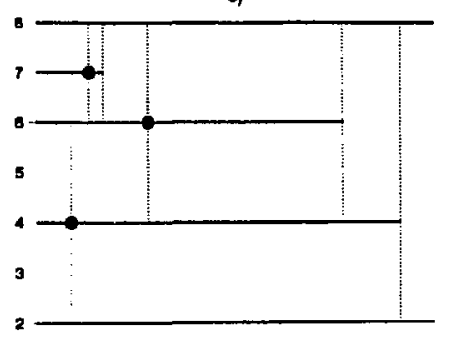

$\begin{array}{llllllllllll}0.0 & 0.1 & 0.2 & 0.3 & 0.4 & 0.6 & 0.6 & 0.7 & 0.8 & 0.6 & 1.0\end{array}$ INTERACTION $\mathbf{J} / \mathrm{t}$ 
Figure 3.10. Total number of states in the ground-state manifold and in the low-lying excitations within 0.1 I! I of it for the sc $\left(t^{\prime}<0\right)$ lattice and $N=3$.

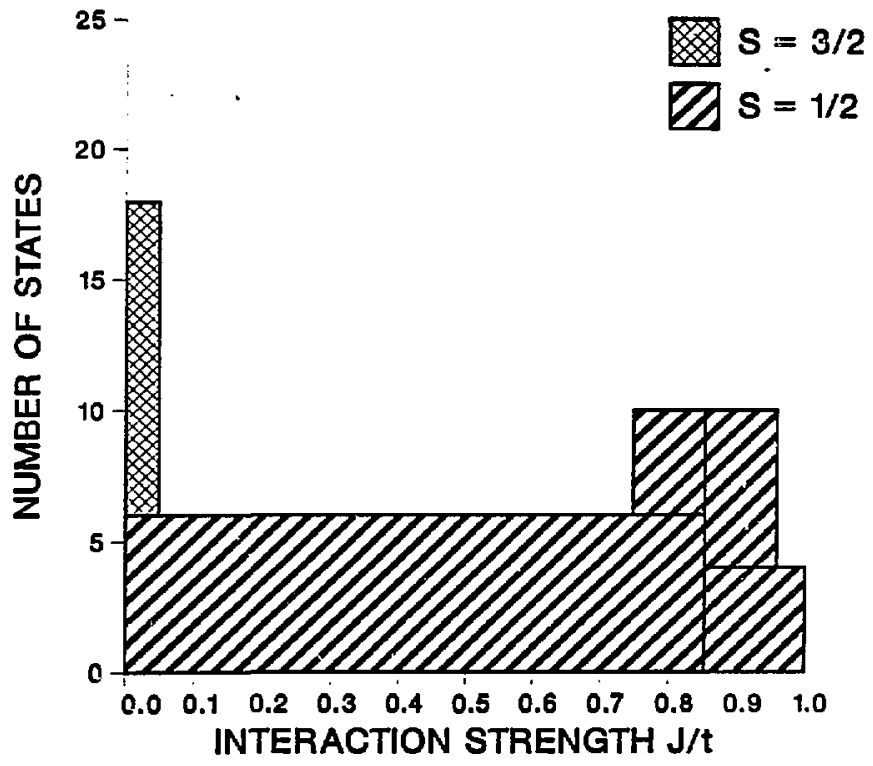


Figure 3.11. Total number of states in the ground-state, manifold and in the low-lying excitations within $0.1|t|$ of it for the $f c c(t>0)$ 1attice and $N=7$. There are no low-lying excitations in the range $0.1<J / t<1.0$. The spin-pileup effect can be seen at $J=0$.

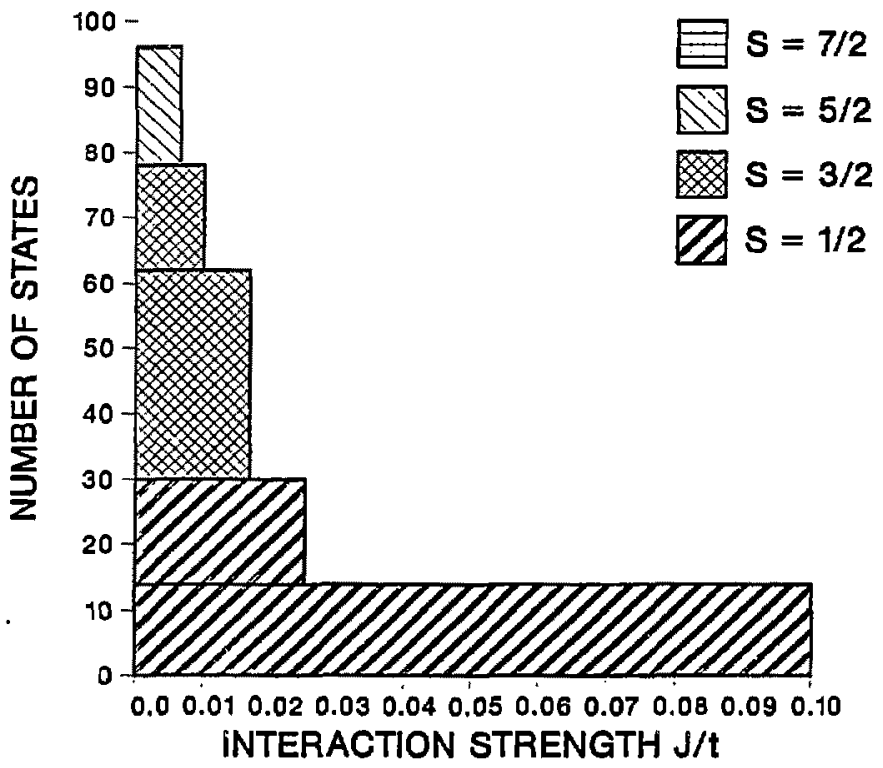


Figure 3.12. Total number of states in the ground-state manifold and in the low-lying excitations within $0.1|t|$ of it for the $f c c(t<0)$ lattice with $r=0$ and $N=7$. There are no low-lying excitations in the range $0.5<J /|\mathrm{l}|<1.0$.

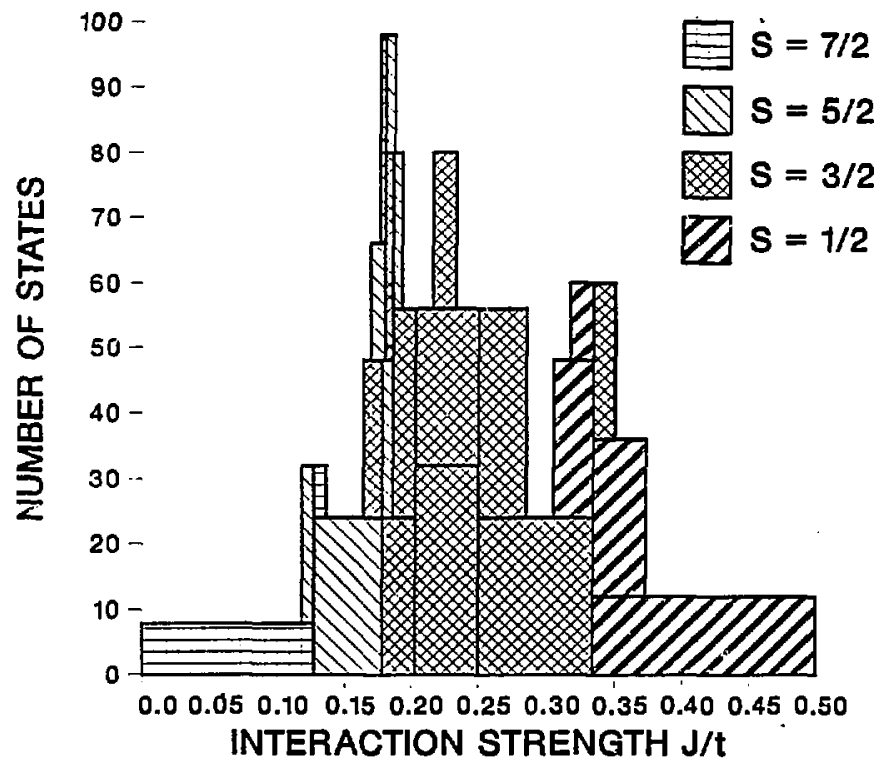


Chapter IV: Exact Solutions of Frustrated Ordinary and Chiral Eight-Site Hubbard Models

\section{IV.1 Introduction}

Although the ground states of the Mott-insulating progenitors of high-temperature superconductors display long-range antiferromagnetic order, it has been suggested that hypotherical "spin-liquid" or "resonating-valence-bond" states which are translationally and spin-rotationally invariani may hold the key to the mechanism of oxide superconductivity ${ }^{1-3}$. These states possess shor-range spin correlations which mimic the shor-range spin comelations in a singlet superconductor, and may therefore serve as an appropriate starting point for understanding the nature of superionduetivity in doped Mott insulators. The excitation spectrum of these spin liquids is supposed to contain quasiparticles with reversed spin-charge-relations - the neutral spin-1/2 "spinon" and the spinless charge-e "holon" - and the superconducting ground state is thought of as a condensate of holons.

Despite the theoretical interest in spin liquids, it has proven dinficult to identify a particular frustrated spin-1/2 Heisenberg model with a spin-liguid ground state. The search for these states is complicated by the difficulty of solving frustrated spin models on infinite lattices (or lattices which are large enough to render boundary effects unimportant), especially in view of the possibility of incommensurable spin-density-wave ground states. The understanding of spin liquids is therefore largely based on approximate methods which fall into three different categories: (a) mean-field theories; (b) variational and projection technigues; and (c) small-cluster (finite-system) exact calculations.

\section{(a) Mean-Field Theories.}

Conventional mean-field theory for antiferromagnets (AF), in which the spin at every site acquires a nonzero expectation value, is not appropriate for the study of spin 
liquids, since they are by hypothesis spin-rotationally invariant. Affleck and Marston ${ }^{4}$ have proposed an altemative mean-field theory by recalling that antiferromagnetic excharge between two spins at sites $i$ and $j$ arises from the virtual hop of an electron from site $i$ to site $j$ and back again, viz., $\left[-2 J_{i j}\left(c_{i \sigma}^{\dagger} c_{j \sigma}\right)\left(c_{j \tau}^{\dagger} c_{i \tau}\right)\right]$. This expression leads naturally to the introduction of the (possibly complex) link variables $x_{i j} \equiv\left(J_{i j} / 2\right)<c_{j}^{\dagger} c_{i \sigma}>$ and the corresponding mean-field Hamiltcaian

$$
H_{M F}=\sum_{(i, i)} \frac{\left|\chi_{i j}\right|^{2}}{J_{i j}}+\sum_{(i, j), \sigma}^{n}\left[\chi_{i j} c_{i \sigma}^{t} c_{j \sigma}+\chi_{i j}^{*} c_{j \sigma}^{\dagger} c_{i \sigma}\right] .
$$

The mean-field state is obtained by minimizing $H_{M F}$ with respect to the link variables $\chi_{i j}$, a procedure which yields exact results for the $S U(N)$ Heisenberg model in the iarge- $N$ linnit. The ground state in the mean-field approximation is then the Slater determinant obtained by filling the single-particle states in the lower half of the specrum of (4.1). Since the effective hopping amplitudes $\chi_{i j}$ are generally complex, the Hamiltonian (4.1) is equivalent to a system of noninteracting fermions moving in a "magnetic field" which couples only to orbizal motion. The mean-field Hamiltonian (4.1) preserves an important symmerty of the Heisenberg model, naracly the local $U$ (1) gauge symmetry associated with the conservation of particie number 2i each site ${ }^{5}$. Under a local gauge transformation, $c_{j \sigma}^{\dagger} \rightarrow \exp \left(i \Lambda_{j}\right) c_{j \sigma}^{\dagger}$, evtry stat: in the Hilbert space of the Heisenberg model is multiplied by the same overall phase factor $\exp \left(i \Sigma_{j} \Lambda_{j}\right)$ and therefore all observables are unafiected.

In general $H_{M F}$ is minimized ${ }^{6}$ by states with nonzero $\chi_{i j}$ only on isolated links of the lattice. To obtain stable translationally invariant spin-liquids in a mean-field approximation one may intruduce biquadratic spin-spin interactions ${ }^{4}$ which suppress fluctuations of the magnitudes $\left|\chi_{i j}\right|$. The resulting states have uniform (bun fluctuating) charge density. 


\section{(b) Variationll and Projection Techniques.}

Slater determinants such as the mean-field states described above can be converted into suitable variational wavefunctions for the Heisenberg model by progressively eliminating those components of the Slater determinant which correspond to multiple occupancy of sites - the "Gutzwiller" technique. These projected wavefunctions yield excellent variational energies ${ }^{7}$ (and therefore accurately describe short-range correlaions) when the "flux" through every elementary plaquette (ijkl) is $\pi$, i.e., when the phase of the product $\chi_{i j} \chi_{j k} \chi_{k i} \chi_{d i}$ of the link variables around the plaquette is $\pi$. On a square lattice with diagonal (frustrating) interactions, the optimal state (with uniform $\left(\chi_{i j} \mid\right)$ is the "chiral" state 3,8 , with flux $\pi / 2$ through each elementary triangle, which breaks both time-reversal and parity symmetries.

The philosophy behind the Gutzwiller approach is a familiar one. To study a strongly interacting many-body system, one first identifies a simpler weak-coupling limit which embodies the same symmetries, and then imagines a smooth deformation of this soluble model into the intractable Hamiltonian under consideration. If no phase Jansition or level crossing intervenes, then the two opposite limits will be qualitatively similar. In some cases, the smooth continuation from weak to strong coupling can be convincingly demonstrated. An instructive example of such a continuum of models is the half-flled, square-lattice Hubbard model with nearest-neighbor-only hopping ${ }^{9}$. In the small Hubbard $U$ limit, ths system is a commensurable spin-density-wave insulator, with an exponentially small charge gap. In the opposite (large Hubbard $U$ ) limit, charged excitations can be formally eliminated, resulting in a nearest-neighbor Heisenberg AF with a Neel-ordered ground state. In both cases, the ground-state density correlations decay exponentially, and the low-energy, long-wavelength excitations are gapless antiferromagnons. Despite the apparent conceptual difference between a commensurable spin-density-wave insulator (whose charge gap is caused by a doubling of the unit cell), and a Neel-ordered Mott insulator (whose gap is generally viewed as a 
many-body effect), there appears to be no phase-boundary separating them. The use of Gutzwiller-projected wavefunctions tacilly assumes a smooth interpolation of ground states from weak to strong coupling. If these two limits can be continuously related, the Gutzwiller appsuach provides a crude but powerful approximation for discussing strongly interacting problems using weak-coupling methods.

If the proposed spin-liquid states do indeed exist as ground states of an appropriately frustrated spin model, one may ask whether or not a smooth continuation to a more easily studied weak-coupling model exists. In particular, the mean-field theory of equation (4.1) suggests $^{10}$ a clear possible starting point: a Hubbard model in the presence of an arbitrary magnetic field which couples only to orbital motion, i.e.,

$$
\begin{gathered}
H=-\sum_{i, j, 0} t_{i j} c_{i \sigma}^{\dagger} c_{j \sigma}+U \sum_{i} n_{i \uparrow n_{i \downarrow}}, \\
s_{i j}=r_{i j} e^{i \phi_{i j}} \quad ; \quad t_{i j}=r_{j i}^{*},
\end{gathered}
$$

where $n_{j \mathrm{o}}=c_{i \mathrm{\sigma}}^{t} c_{i \sigma}$ is the particle number at site $i$, the $T_{i j}$ are real and positive, and hopping is not limited to nearest-neighbors. At half-filling and in the large- $U$ limit, for any choice of link phases $\left\{\phi_{i j}\right\}$, this Hubbard model approaches a frustrated spin$1 / 2$ Heisenberg model in which the ratios $J_{i j} / J_{k d}$ are simply $\left(T_{i j} / T_{k J}\right)^{2}$. Each set of link phases therefore specifies a family of Hamiltonians which interpolates between different soluble models $(U=0)$ and one intractable spin mndel $(U=\infty)$. If for a sufficiently clever choice of phases it is possible to interpolate smocthly between the two limits, then one can infer properties of the frustrated Heisenberg model from a careful study of tight-binding independent-particle models.

For a generic choice of hopping phases $\phi_{i j}$, the model described by the Hamiltonian (4.2) explicitly breaks time-reversal and parity symmetries. For large $U$ this is reffected in the fact that the corresponding Heisenberg model includes three-spin interactions ${ }^{10}$

$$
\sum_{i j k} \frac{T_{i j} T_{j k} T_{k i}}{U^{2}} \sin \left[\Phi_{i j k}\right] S_{i} \cdot S_{j} \times S_{k}
$$


where $\Phi_{i j k} \equiv \phi_{i j}+\phi_{j k}+\phi_{k i}$ is the flux through triangle $i j k$. These terms vanish in the Mott limit, since they are smaller by a factor of $(T / U)$ than the usual quadratic spinspin Heisenberg coupling $\left[\left(4 T_{i j}^{2} / U\right) S_{i} \cdot S_{j}\right]$. Thes may, however, act as infinitesimal symmetry-breaking fields (in the large- $U$ limit) if the ground state of the corresponding frustrated Heisenberg model spontaneously breaks time-reversal or parity symmetries.

Of course, a continuous family of models does not ensure that the corresponding states will vary smoothly, since a phase transition could (and frequently will) intervene. A necessary condition for the absence of a phase transition between the large and small- $U$ limits is that both limiting states must have the same symmetry (nocrossing rule). For example, weakly frustrated square-lattice antiferromagnets are thonght to have Neel-ordered ${ }^{11-13}$ ground states, so one cannot expect the (paramagnetic) ground states of ight-binding models (4.2) with weak second-neighbor hopping to continue smoothly as $U$ is increased. A second requirement for continuity between a small- $U$ state and a Mott insulator is that the small- $U$ state must be locally neurral with a gap in the charged excitation spectrum (insulator rule). This condition ensures that a metal-insulator transition does not interrupt the continuation process.

Which link phases $\phi_{i j}$ are most likely to pernit continuation from a (paramagnetic) Slater determinant to a translationally invariant Mott insulator? The similarity between the generalized Hubbard model (4.2) and the mean-field theory (4.1) suggests distributions of flux which correspond to mean-field solutions with uniform magnitudes $\left|\chi_{i j}\right|$. The corresponding Slater determinant will then have spin-correlations which should closely resemble its large- $U$ cousin, facilitating a smooth interpolation between the two states. It is also necessary to have a single-paricle gap at $U=0^{+}$, to satisfy the insulator rule. To obtain a translationally invariant insulator the charge density must also be uniform and the current on each link must vanish. This latter condition is simply the statement that the experazsion value of the Hamiltonian is stationary with 
respect to varying the link phases, which is automatically satisficd by choosing fluxes corresponding to a uniform-amplitude mean-field state.

A strong candidate for adiabatic continuation (from a tight-binding model to a spin-liquid) in the strongly frustrated regime is then the chiral Hubbard model ${ }^{\mathbf{3 , 8}}$ with $\pi / 2$ flux per triangle. It has a spin-singlet translationally invariant ground state that breaks time-reversal and parity (satisfying the no-crossing rule) and the presence of $\pi$ flux per plaquette doubles the (magnetic) unit cell opening a "chiral gap" to singleparticle excitations for nonzero next-nearest-neighbor hopping (satisfying the insulator rule).

(c) Exact Diagonalization of a Periodic Small-Cluster Hamiltonian.

The approach used in this contribution is the small-cluster approximation ${ }^{14}$ which consists in the exact diagonalization of the generalized Hubbard Hamiltonians (4.2) applied to a small cluster with periodic boundary conditions (PBC). The small-cluster afproach begins with the periodic crystal approximation, ${ }^{15}$ modeling a bulk crystal by a lattice of $M$ sites with PBC. Standard approaches ${ }^{16}$ take the thermodynamic limit $(M \rightarrow \infty)$ of the noninteracting system (sampling a continuum in momentum space that spans the Brillouin 20ne) and treat the subsequent electron-correlation effects in an approximate manner. The small-cluster approach fixes the number of lattice sites to be small (restricting the momentum-space sampling to a coarse grid of high-symmetry points) but solves exactly for all electron-correlation effects. The one-electron band structure of both methods is identical at the sampled wavevectors. The relationship of the many-body solutions (at equal electron concentration) for the macroscopic crystal and the small cluster is much more complicated because of uncontrolled finite-size effects in the lamer. However, the small-cluster approach provides a rigorous and complimentary method to study the many-body problem that may be extrapolated to macroscopic crystals. 
The small-cluster approach was proposed independently for the Hubbard model by Harris and Lange ${ }^{17}$ and Falicov and Harris ${ }^{18}$ with the exact solution of the two-site cluster. The solution of the four-site square (and tetrahedral) cluster ${ }^{19}$ marked the first time that group theory was used to factorize the Hamiltonian (4.2) into block-diagonal form by using basis functions of definite spin that transform according to the irreducible representations of the full space group. Recent work has concentrated on moderately sized $(M \leq 20)$ square-lattice clusters ${ }^{20,21}$. A brief history of applications of the small-cluster approach to different geometries and real materials can be found in Ref. 21.

This contribution examines the ground-state symmetry, wavevector, spin, and correlation functions for the ordinary and the chiral Hubbard models at half-filling on an eight-site square-lattice cluster as functions of the interaction strength $U$ and of the hopping parameters $t$ and $t$. Section IV.2 discusses the symmetries of the two models and the method of calculation; Section IV.3 includes the results for the ground states of both models and their properties; the final section presents the conclusions and suggestions for further work.

\section{TV.2 Symmetries and Calculational Methods}

Two different eight-site square-lattice clusters are illustrated in real and reciprocal space in Figs. 4.1 and 4.2: the "ordinary" Hubbard model 22 and the "chiral" Hubbard model ${ }^{3.8}$. Both models have hopping amplinudes with the same magninudes for the nearest-neighbor hopping $(t)$ and for the next-nearest-neighbor hopping $(r)$, respectively, but differ in the relative phases of the hopping parameters. The Hubbard model has all real hopping matrix elements whereas the chiral Hubbard model has relative phases chosen so that each fundamental triangle contains a "flux" of $\pi / 2$ (in units where one flux quanturn $=2 \pi$ ). Tables 4.1 and 4.2 summarize the nonzero hopping matrix elements $t_{i j}$ for the two models in terms of the parameters $t$ and $t$. One 
should note that the factor of two multiplying the next-nearest-neighbor hopping matrix elements arises from a renormalization of the hopping parameters caused by the PBC (the four next-nearest-neighbors of an odd [even] site $i$ are two each of the remaining odd [even] sites except for the site $i \pm 4$ ).

(i) Number operator.

The total-number operator for each spin $N_{\sigma}=\Sigma_{i} n_{i \sigma}$ commutes with the Hamiltonian in equation (4.2) and is a conserved quantity. The many-body states may be labeled by the total number of electrons $N=N_{\uparrow}+N_{\downarrow}$.

(ii) Spin symmetry.

The total 2 -component of $\operatorname{spin} S_{z}=\frac{1}{2}\left(N_{\uparrow}-N_{\downarrow}\right)$, formed from the difference of these number operators, is the third component of an iniernal SU(2) spin symmetry with raising and lowering operators, $S_{+}=\Sigma_{i} c_{i} \dagger c_{i \downarrow}$ and $S_{-}=\left(S_{+}\right)^{\dagger}$, that commute with the Hamiltonian (4.2)

$$
\left[S_{+}, S_{-}\right]=2 S_{z},\left[S_{z}, S_{ \pm}\right]= \pm S_{ \pm},\left[H, S_{ \pm}\right]=\left[H, S_{z}\right]=0
$$

These commutation relations imply that the square of the total-spin operator $S^{2}=\frac{1}{2}\left(S_{+} S_{-}+S_{-} S_{+}\right)+S_{2}^{2}$ also commutes with the Hamiltonian, so the many-body states may be labeled by their total spin $S$ and total $z$-component of spin $m_{S}$, with every state in a given spin multiplet degenerate in energy.

(iii) Pseudospin symmetry.

Another internal SU(2) "pseudospin" symmetry can be found in special cases. ${ }^{23-25}$ The 2 -component of pseudospin is given by $J_{z}=\frac{1}{2}(N-M)$. As seen above it commutes with tie Hamiltonian; it also satisfies an SU(2) algebra 


$$
\left[J_{+}, J_{-}\right]=2 J_{2} \quad, \quad\left[J_{1}, J_{ \pm}\right]= \pm J_{ \pm},
$$

with the pseudospin raising and lowering operators: $J_{+}=\Sigma_{j} \exp \left(i \theta_{j}\right) c_{j}^{\dagger} c_{j}^{\dagger}$ and $J_{-}=\left(J_{+}\right)^{\dagger}$. Although the latter do not commute with the Hamiltonian, they become raising and lowering operators of the Hamiltonian,

$$
\left[H, J_{ \pm}\right]= \pm U J_{ \pm}
$$

whenever the phase condition

$$
t_{i j}=-e^{i\left(\theta_{i}-\theta_{j}\right)} t_{i j}^{*}
$$

is satisfied, or equivalently

$$
\theta_{i}-\theta_{j}=2 \phi_{i j}+\pi(\bmod 2 \pi)
$$

is satisfied. If (4.6) holds, then the square of the pseudospin operator $J^{2}=\frac{1}{2}\left(J_{+} J_{-}+J_{-} J_{+}\right)+J_{z}^{2}$ commutes with the Hamiltonian and is another conserved quantity. The phase condition can be satisfied whenever the hopping matrix is bipartite (i.e., when there are two disjoint sublattices $A$ and $B$ with nonzero hopping between $A \leftarrow B$ only) by the choice

$$
\theta_{i}=\left[\begin{array}{ll}
0, & i \in A \\
\pi, & i \in B
\end{array}\right.
$$

Equation (4.7) holds for the ordinary Hubbard model when $t=0$, but cannot be satisfic otherwise. The phase condition (with the phase choice of Eq. 4.8 ) is always satisfied for the chiral Hubbard model when one chooses a gauge that is real for $A \longleftrightarrow B$ sublattice hopping, and imaginary for $A \longleftrightarrow A$ and $B \hookleftarrow B$ sublattice hopping. The many-body states for the half-filled band $(N=M)$ all have $J_{z}=0$. In the case of the chiral Hubbard mociei they may also be labeled by their pseudospin $J$. 
(iv) Space operations for the ordinary Hubbard model.

The space group of the ordinary Hubbard model is a symmorphic, moderately sized finite group constructed from the $C_{4 v}$ point-group operations and the eight translation vectors of the lattice: the four nearest-neighbor translations are denoted by $\tau$; the two next-nearest-neighbor translations by $\theta$; and the one third-nearest-neighbor translation by $\Omega$. The space group is of onder 64 and is composed of 16 classes. The Brillouin zone $^{26}$ (see Fig. 4.1) is sampled at four symmery stars: $\Gamma(\mathrm{d}=1) ; M(\mathrm{~d}=1) ; X(\mathrm{~d}=2)$; and $\Sigma(d=4)$. The character table ${ }^{26}$ is reproduced in Table 4.3.

(v) Site-permutation operations for the ondinary Hubbard model.

There is a larger group, a cluster-permutation group, that includes the space group as a subgroup and is generated by the space-group generators plus a permutation operator that cornmutes with the Hamiltonian but is not a space-group operation. ${ }^{27}$ In general, this extra permutation operator may be constructed from a set of transpositions (pair interchanges): the origin is interchanged with the site that is farthest away from it. The remaining sites are also pairwise interchanged (if necessary) so that the origival neighbor structure of the cluster is preserved. The resultant permutation operator is a nonrigid mapping of the lattice onto itself and, therefore, is not an element of the space group. For example, the nearest-neighbors of site- 1 are the sites $2,4,6$, and 8 (see Fig. 4.1) and the next-nearest-neighbors are the sites 3 and 7. Site-5 has an identical neighbor structure, so the permutation operator $P$ that interchanges site- 1 and site-5 will commute with the Hamiltonian but it is not simply a combination of translations and point-group operations and hence is not a space-group operation. A similar permutation operator has been found for other clusters, e.g. a ten-site square-lattice cluster. ${ }^{27}$ The existence of this nontrivial permutation operator is a finite-size effect of periodic elusters since an infinite system does not have any symmetry beyond that of the space group. It also depends strongly on the geometry of the system since every 
finite cluster does not necessarily have this extra "hidden" symmetry.

The cluster-permutation group is composed of 128 elements divided into twenty classes and recorded in Table 4.4. Note that the presence of the permutation operator $P$ forces physically different space-group operations (such as the translations, rotations and reflections) to be sometimes in the same class. The character table is reproduced in Table 4.5 and includes the compatibility relations between representations of the cluster-permutation group and the real space-group in the last column.

The group of translations forms an abelian subgroup of the cluster-permutation group, but it is not an invariant subgroup. This means that one cannot build representations of the cluster-permutation group in the ordinary manner ${ }^{28}$ for a space group and, in particular, there are some representations that require essential degeneracies between states that have different wavevectors. Such is the case for representations $\phi_{1}$, $\phi_{2}, \phi_{3}$, and $\phi_{4}$ in Table 4.5 .

(vi) Space operations for the chiral Hubbard model.

The fixing of gauge for the chiral Hubbard model drastically reduces the symmetry of the Hamiltonian. The crystal structure becomes a rectangular lattice with a basis of two atoms (see Fig. 4.2); there are no fourfold rotations or mirror planes. The space group is a symmorphic group constructed from the $C_{2}$ point-group operations and the four translation vectors of the lattice. It is of order 8 and composed of 5 classes. The Brillouin zone $e^{26}$ (see Fig. 4.2) is sampled at three symmetry stars: $\Gamma(d=1) ; X(d=1)$; and $\Sigma(d=2)$. The character table ${ }^{26}$ is reproduced in Table 4.6 .

There are no finite-size-effect permutation operators that commute with the Hamiltonian for the chiral Hubbard model. This is because the preservation of the neighbor structure of the cluster is not a sufficient condition for a permutation operator to commute with the Hamilonian if the phases in the hopping matrix are not uniform. 
(vii) Gauge-space operations for the chiral Hubbard model.

There is a larger group, a gauge-space group, that includes the space group as a subgroup and is composed of rotations and translations followed by gauge transformations. The gauge transformations $\chi$ are unitary operators of order 2 (i.e., square to the identity); they are composed of products of the single-site gauge transformations

$$
G_{i} \equiv\left(1-2 n_{i \uparrow}\right)\left(1-2 n_{i \downarrow}\right), G_{i}^{\dagger}=G_{i}^{-1}=G_{i}, G_{i}^{2}=1,
$$

which change the sign of the electron creation and annihilation operators

$$
G_{i} c_{j \sigma}^{\dagger} G_{i}=(-1)^{\delta_{i j}} c_{j \sigma}^{\dagger} \quad, G_{i} c_{j \sigma} G_{i}=(-1)^{\delta_{i j}} c_{j \sigma},
$$

at the corresponding atomic site. The uniform gauge transformation $\bar{E}$

$$
E=\prod_{i=1}^{8} G_{i}
$$

changes the sign of the creation and annihilation operators at every site and, acting on state vectors, equals $1[-1]$ when the number of electrons is even [odd]. The full group, which allows $\bar{E}$ to be 1 or [-1], is discussed in the appendix. The case with $E=1$ is discussed here since the half-filled band contains an even number $(N=8)$ of electrons. In this case, the gauge-space group requires three nontrivial gauge transformations

$$
\text { - } \chi_{1}=G_{3} G_{7}, \chi_{2}=G_{4} G_{8}, \chi_{3}=G_{1} G_{4} G_{5} G_{8}
$$

in its group elements. The group is generated by a fourfold rotation followed by a gauge transformation (gauge-rotation) $-\chi_{1}\left\{C_{4} \mid 0\right\}$, the translation from site-1 to site-2 - $\left\{E \mid \tau_{2}\right\}$, and a translation from sice-1 to site-4 followed by a gauge transformation (gauge-translation) $-\chi_{3}\left(E \mid \tau_{4}\right)$. The gauge-space group, which commutes with the chiral Hubbard model Hamiltonian, is composed of 32 elements divided into 11 classes and recorded in Table 4.7. The character table is reproduced in Table 4.8. 
The group of translations and gauge-translations forms an abelian invariant subgroup of the gauge-space group; therefore Bloch's theorem ${ }^{29}$ holds with gauge-wavevectors ${ }^{30}$ distributed in a gauge-Brillouin zone (see Fig. 4.2). The gaugeBrillouin zone is sampled at four symmetry stars: $\gamma(d=1) ; m(d=1) ; x(d=2)$; and $\sigma(d=4)$. Lower-case letters are used to denote the gauge-wavevectors; the compatibility relations between representations of the gauge-space group and the real space group have been included in the last column of Table 4.6. The gauge-space group is isomorphic to the space group of an eight-site square lattice with point group $C_{4}$, which is physically sensible since the "magnetic field" is uniform.

(viii) Particle-hole parity operator.

A particle-hole parity operator 23,31

$$
R=G_{1} G_{3} G_{5} G_{7} \prod_{i=1}^{8}\left(c_{i}^{\dagger}+c_{i \uparrow}\right)\left(c_{i \downarrow}^{\dagger}+c_{i \downarrow}\right)
$$

is constructed out of the $B$-sublattice gauge transformation and the operator that interchanges particles with holes. The particle-hole parity operator is unitary and squares to one so its eigenvalues are \pm 1 . It satisfies a commutation relation with the Hamiltonian (4.2)

$$
[H, R]=2 U J_{z} R
$$

whenever the hopping matrix $t_{i j}$ obeys

$$
\begin{aligned}
& t_{i j}=t_{j i} \text { for } i \in A, j \in B \text { or } i \in B, j \in A \\
& t_{i j}=-t_{j i} \text { for } i \in A, j \in A \text { or } i \in B, j \in B .
\end{aligned}
$$

The particle-hole parity operator commules with the Hamiltonian if condition (4.15) is satisfied and $J_{z}=0$ (half-filled band). Condition (4.15) holds for the ordinary Hubbard model only when $r=0$ but always holds for the chiral Hubbard model (with the chosen gauge). The particle-hole parity operator anticommutes with the z-component 
of spin $\left(R, S_{z}\right\}_{+}=0$ so it may be used as an additional symmetry label for the manybody states of the chiral Hubbard model with $m_{s}=0$.

An interesting characteristic of the chiral Hubbard model is that it retains all of the "special" parameter-independent symmetries (pseudospin and particle-hole parity) of the nearest-neighbor Hubbard model on a bipartite lattice even when next-nearestneighbor hopping introduces frustration. The chiral Hubbard model also does not have the finite-size effect of extra permutation symmetries that are not space-group (gaugespace-group) symmetries.

(ix) Parameter-dependent symmetries.

In addition to the parameter-independent symmetries there are two classes of parameter-dependent symmetries. The first class is geometrical and depends on the ratio $t^{\prime} / t$. When $t / t=0$, the Hubbard model on an eight-site square-lattice cluster is identical to the Hubbard model on an eight-site body-centered-cubic-lattice cluster. ${ }^{21}$ When $t / t=1 / 2$, the renormalized hopping to nearest-neighbors equals the renormalized hopping to next-nearest-neighbors and the eight-site square-lattice cluster becomes an eight-site triangular-lattice cluster which is, in turn, identical to an eight-site facecentered-cubic-lattice cluster. In this second case, the large $U$ solutions are known to be threefold degenerate at half-filling. ${ }^{13,21}$ There are no geometrical degeneracies for the chiral Hubbard model because the hopping matrix elements have nonzero phases.

The second class is dynamical and depends on the interaction parameter $U$. The first two of an infinite class of conserved currents have already been found ${ }^{32.33}$ for the nearest-neighbor Hubband model in one-dimension. Almost certainly additional currents exist for finite clusters since any finite cluster can be mapped onto a onedimensional ring with hopping terms that extend beyond nearest-neighbors, but these currents hav? not been determined for either of the models considered here. 


\section{(x) Simultaneous-eigenvector symmetry.}

Finally, there is a whole class of eigenstates of the Hamilionian (4.2) whose structure (although not necessarily their energies) is independent ${ }^{23,25}$ of the interaction parameter $U$. This occurs whenever an eigenstate of the kinetic energy is simultaneously an eigenstate of the interaction term. An example is the fully polarized ferromagnetic state which is annihilated by both the kinetic and potential energy operators. Such phenomenon does not often occur and it is not pursued further here.

The total number of independent many-bcdy states for the half-filled band of an eight-site cluster is 12,870 . The systematic application of group theory is used to reduce the size of the Hamiltonian matrix. The grand orthogonality theorem and the matrix-element theorem ${ }^{34-36}$ (generalized Unsbld theorem) guarantee that the Hamiltonian matrix will be in block-diagonal form, with no mixing between states of different spin or spatial symmetry, when it is expanded in a symmetrized basis that has definite spin and transforms according to the $(1,1)$ matrix elements of an irreducible representation of the symmetry group. Neither the additional pseudospin, nor the particle-hole parity symmetries (which produce a furthrs reduction of the block sizes for the chiral Hubbard model), nor any of the parameter-dependent symmetries were utilized. Use was made of a symmetry-adapted computer algorithm ${ }^{21}$ that calculates the $(1, !)$ matrix elements ${ }^{37}$ of the irreducible representations, constructs projection operators from these matrix elements, and operates on maximum $z$-component of spin states $\left(m_{S}=S\right)$ to generate symmetrized basis functions of definite spin and spatial symmetry. The Hamiltonian blocks are determined exactly in this symmetrized basis (incorporating multiple-precision integer arithmetic ${ }^{38}$ when necessary) and are checked for completeness within each subspace of definite spin and spatial symmetry. The resultant blocks are diagonalized by the so-called QL algorith ${ }^{39}$ (which determines all of the eigenvalues and eigenvectors) when the blocks were smaller than $100 \times 100$ and by Cullum and Willoughby's single-vector Lanczos routines ${ }^{40}$ (which determine 
the lowest five eigenvalues and eigenvectors) for the larger blocks. Tables 4.9 and 4.10 summarize the reduced block sizes for the ordinary Hubbard model and for the chiral Hubbard model. The applisation of group theory reduces the block sizes by a factor of $\mathbf{5 0}$ which, in turn, reduces the diagonalization time by five orders of magnitude.

\section{IV.3 Results}

The specrum of a generalized Hubbard Hamiltonian (4.2) is independent ${ }^{23,41}$ of the sign of $t$ or $t^{\prime}$ for the half-filled band, so only the cases with $t \geq 0$ and $t \geq 0$ were considered. In the strong-interaction limit $(U \rightarrow \infty)$, both the Hubbard model and the chiral Hubbard model approach the same frustrated Heisenberg antiferromagnet ${ }^{10.17,42}$ with exchange integrals $J=4 t^{2} / U$ (nearest-neighbor) and $J^{\prime}=4 t^{2} / U$ (next-nearestneighbor). Therefore, the ground-state phase diagrams for both models are expected to be identical to second order $O\left(t^{2} / U\right)$ although at higher order they need not be the same. It is known' that the ground state of the nearest-neighbor Hubbard model $\left(t^{\prime}=0\right)$ is a spatially uniform spin-singlet that may be adiabatically continued from a spin-density wave insulator $\left(U=0^{+}\right)$to a two-sublattice Nél antiferromagnet ${ }^{11}$ $(U \rightarrow \infty)$ without level crossings. The eight-site frustrated Heisenberg model has been studied for all values of $J$ and $J$ in Ref. 13. It undergoes a levei crossing from a two-sublattice NEel AF to a four-sublattice NEel AF, with a threefold degeneracy at the level crossing as $J^{\prime} / J$ increases.

There are four different ground-state correlation functions that were computed in order to determine the ground-state properties: The spin-spin correlation function $\left(L_{i}\right)$; the z-component of spin-z-component of spin correlation function $\left(M_{i}\right)$; the numbernumber correlation function $\left(N_{i}\right)$; and the spin-triple-product correlation function $\left(O_{124}\right)$. The correlation functions are defined by the following ground-state expectation values: 


$$
\begin{gathered}
L_{i}=\frac{1}{v_{j}} \quad \sum_{(j k)=i \text { th NN }}\left\langle G S\left|S_{j} \cdot S_{k}\right| G S\right\rangle, i=0,1,2,3 ; \\
M_{i}=\frac{1}{v_{i}} \quad \sum_{(j k)=i \text { th NN }}\left\langle G S\left|S_{j}^{2} S_{k}^{2}\right| G S\right\rangle, i=0,1,2,3 ; \\
N_{i}=\frac{1}{v_{i}} \quad \sum_{(j k)=i \text { th NN }}\left\langle G S\left|n_{j} n_{k}\right| G S\right\rangle, i=0,1,2,3 ; \\
O_{124}=\left\langle G S\left|S_{1} \cdot S_{2} \times S_{4}\right| G S\right\rangle ;
\end{gathered}
$$

where $i$ denotes the distance between site- $j$ and site- $k$ and $v_{i}$ denotes the number of site-pairs separated by this distance. The correlation functions satisfy three sum rules:

$$
\begin{gathered}
\sum_{i=0}^{3} v_{i} L_{i}=S(S+1) ; \\
\sum_{i=0}^{3} v_{i} M_{i}=m_{S}^{2} ; \\
\sum_{i=0}^{3} v_{i} N_{i}=N^{2}=64 ;
\end{gathered}
$$

since the ground state has definite total-spin and definite electron number. Furthermore, the spin correlation functions are related by

$$
L_{i}=3 M_{i}, \text { for } S=0,
$$

i.e., whenever the ground state is a spin-singlet. The sum rules (4.17) and the relation (4.18) hold for all the correlation functions that were calculated. The spin-tripleproduct correlation function is formed from three spins that lie on the vertices of a right triangle consisting of two nearest-neighbor pairs and one next-nearest-nuighbor pair. This is the cnly (potentially) nonzero spin-triple-product correlation function for an eight-site cluster.

The maximum hopping integral $T=\max \left(t, t^{\prime}\right)$ was chosen as the unit of energy and the hopping parameters were selected in the range

$$
0 \leq \frac{2 r^{\prime}}{t+2 t^{\prime}} \leq 1 \text {, }
$$


from pure nearest-neighbor hopping $\left(r^{\prime}=0\right)$ to pure next-nearest-neighbor hopping $(t=0)$. The interaction strength was varied in the range

$$
0 \leq \frac{U}{4 t+4 r^{\prime}+U} \leq 1
$$

from the noninteracting regime $(U=0)$ to the strong-coupling limit $(U=\infty)$.

(i) Ordinary Hubband Model.

The one-electron band structure of the Hubbard model consists of four levels: $\Gamma_{1 p}$ (degeneracy $d=1$; energy $\left.\varepsilon=-4 t-4 r^{\prime}\right) ; \Sigma_{1 p}(d=4 ; \varepsilon=0) ; X_{1 p} \quad\left(d=2 ; \varepsilon=4 r^{\prime}\right)$; and $M_{1 p}\left(\mathrm{~d}=1 ; \varepsilon=4 t-4 t^{\prime}\right)$. The ground state for the noninteracting $(U=0)$ half-filled band is formed by filling the $\Gamma_{1 p}$ level and placing six electrons in the $\Sigma_{1_{p}}$ level for $r<t$ or by filling the $\Gamma_{1_{p}}$ and $M_{1_{p}}$ levels and placing four electrons in the $\Sigma_{1_{p}}$ level for $t^{\prime}>t$. In either case, the noninteracting half-filled band has a degenerate ground state and would require degenerate perturbation theory to determine the small- $U$ ground state.

The symmetry of the ground state is recorded by attaching the spin multiplicity $(2 S+1)$ as a superscript to the symbol fo: the irreducible representation that transforms according to the many-body state (as given in Table 4.5). The ground-state symmetries are plotted as a function of the relative hopping (4.19) along the vertical axis and of the interaction strength (4.20) along the horizontal-axis in the phase diagram of Figure 4.3. The spin-spin correlation functions $\left(L_{i}\right)$ and the number-number correlation functions $\left(N_{i}\right)$ are recorded in Fig. 4.4 and Fig. 4.5 respectively, for three different values of $f / t$. There are no discontinuities in the correlation functions when there are no level zrossings in the ground state. Note that as $U \rightarrow \infty$ the ground state contains one electron per site and the spins are oriented ${ }^{13}$ into a two-sublatice Neel AF $\left(t^{\prime}<t / 2\right)$ or a four-sublattice NEel AF $(t>t / 2)$. The spin-triple-product correlation function, $O_{124}$, vanishes for all values of $t, t$, and $U$ (since the ground states are 
invariant under parity).

Note that the presence of the cluster-permutation group symmetries $\phi_{2}$ and $\phi_{4}$ as ground states is a finite-size effect that requires particular states with wavevectors $\Gamma$ and $M$, or $M$ and $X$, to be "accidentally" degenerate.

There are only two regimes where the ground state may be adiabatically continued from $U=0^{+}$to $U \rightarrow \infty$ at fixed $\zeta / t$ without any level crossings. At $t=0$ the ground state has ${ }^{1} \Gamma_{1 n}$ symmetry and continuously changes 9 from a spin-density wave insulator $\left(U=0^{+}\right)$to a two-sublattice NEel AF $(U \rightarrow \infty)$. If $t>t$, the ground state also has ${ }^{1} \Gamma_{1 n}$ symmery and continuously changes from another spin-density wave insulator $\left(U=0^{+}\right)$to a four-sublattice Nél AF $(U \rightarrow \infty)$. For any other value of $t^{\prime}$, $0<t^{\prime}<t$, there are level crossings as $U$ increases from zero to infinity.

There is a small region of phase space $(t=t \approx U)$ where the ground state is ferrimagnetic (symmetry ${ }^{5} \Sigma_{2 n}$ ). This region is very sensitive to the ratio of the hopping parameters $t / t$ but is stable for a wide range of the interaction strength, producing the sliver in Fig. 4.3. The nonzero magnetic moment of this state arises from a complicated interaction between spin and orbital angular momentum that becomes favorable when the two hopping parameters and the interaction stiength are all of the same order of magnitude. This mechanism for producing ferimagnetism in a halfflled band is different from Lieb's mechanism, ${ }^{43}$ since the hopping matrix makes the lattice not bipartite. It is similar in the sense that the magnetism is not saturated. To the authors' knowledge, this is the first observation of a nonzero magnetic moment in the square-lattice Hubbard model at balf-filling.

When $t=t / 2$ the square-lattice cluster becomes a face-centered-cubic-lattice cluster and the ground stave has extra degeneracies. The thick-dashed line in Fig. 4.3 corresponds to a ground state with symmetry ${ }^{1} \phi_{2} \oplus^{1} \phi_{4}(d=6)$ that separates the region where the ordering of the small $U$ ground state changes. The chain-dashed line corresponds, for large- $U$, to the threefold degenerate state ${ }^{1} \Gamma_{1 n} \oplus^{1} \Gamma_{1 n} \oplus^{1} \Gamma_{3 n}$, and 
shows that the ordinary Hubbard model ground state is threefold degenerate in the limit $U \rightarrow \infty$ to all orders in $|t|$.

A few cases of accidental degeneraries remain in the many-body spectrum. ${ }^{44}$ Heilmann's numerical methods ${ }^{45}$ were used to search for parameter-independent hidden symmetries that explain these accidental degeneracies but the problem was not completely resolved. ${ }^{46}$

(ii) Chiral Hubbard Model.

The one-electron band structure for the chiral Hubbard model (see the Appendix) consists of four twofold degenerate levels: $\omega_{1}$ (energy $\left.\varepsilon=-\sqrt{8} t\right) ; \omega_{2}(\varepsilon=\sqrt{8} t) ; \omega_{5}$ $\left(\varepsilon=4 t^{\prime}\right)$; and $\omega_{6}\left(\varepsilon=-4 l^{\prime}\right)$. The noninteracting ground state $(U=0)$ for the halffilled band is formed by completely filling the lowest two energy levels. It is nondegenerate with symmetry ${ }^{1} \gamma_{2}$ (see Table 4.8) for all cases except $t=0$ or $t=0$, where the ground state is degenerate. The large $U$ ground state is known ${ }^{13,21}$ to have symmetry ${ }^{l} \gamma_{1}$ everywhere except at the point $t=t / 2$ where the $(U \rightarrow \infty)$ ground state is threefold degenerate $\left({ }^{1} \gamma_{1} \oplus{ }^{1} \gamma_{1} \oplus{ }^{1} \gamma_{2}\right)$. Therefore, the chiral Hubbard model may satisfy the no-crossing rule only at three points: $t=0 ; t=t / 2$; and $t^{\prime}=0$.

The ground-state symmeries are plotted as a function of the relative hopping (4.19) along the vertical axis and of the interaction strength (4.20) along the horizontal axis in the phase diagram of Fig. 4.6. The ground state is always a spin singlet $(S=0)$, a pseudospin singlet $(J=0)$, and has even particle-hole parity $(R=1)$. The spin-spin correlation functions $\left(L_{i}\right)$, the number-number correlation functions $\left(N_{i}\right)$, and the spin-triple-product correlation functions $\left(O_{124}\right)$ are recorded in Fig. 4.7, Fig. 4.8, and Fig. 4.9 respectively, for representative values of $t / t$. As $U \rightarrow \infty$ the ground state contains one electron per site and is oriented ${ }^{13}$ in a two-sublattice Néel AF $\left(t^{\prime}<t / 2\right)$ or a four-sublattice Nél AF $(\ell>t / 2)$ as expected. The case with $t=t / 2$ (Fig. 4.7) is not ordered as a NEel AF, but rather has intermediate-range AF order that may be interpreted as the approximation to a spin-liquid ${ }^{1-3}$ for a finite system. The spin-triple- 
product correlation function does not vanish for the chiral Hubbard model (at finite $U$ ) because of the explicit breaking of time-reversal and parity symmetries in the Hamiltonian (see Table 4.2). The sign of $O_{124}$ changes at the level crossing between the ${ }^{1} \gamma_{2}$ (small $U$ ) and the ${ }^{1} \gamma_{1}$ (large $U$ ) ground state and its magnitude approaches zero.

It is inleresting to note that, as $U$ approaches infinity (at constant $t$ and $r$ ) not only is $O_{124} \rightarrow 0$ but, in addition, the derivative of $O_{124}$ with respect to $(1 / U)$ also approaches zero (except for the case with $t=t / 2$ ). This feature could be understood in terms of a "triple-product" susceptibility if the following facts are taken into account:

1) The derivative of $O_{124}$ with respect to $(1 / U)$ is directly proportional to the expectation value for the ground state for $U \rightarrow \infty$ of

$$
\left[\sum_{\langle i, j, k\rangle} s_{i} \cdot s_{j} \times s_{k}\right]^{2} ;
$$

2) In the cluster examined here all eigenstates in the $U \rightarrow \infty$ limit are independent ${ }^{13}$ of $t$ and $t^{\prime}$;

3) The eigenstates of the Hamiltonian either for $t=0$ or for $r=0$ are eigenstates of $S_{1} \cdot S_{2} \times S_{4}$, with zero eigenvalue because of the conserved chiral symmetry;

4) From 1), 2), and 3) it follows that the expectation value of (4.21) is zero, regardless of the values of $t$ and $t$. Therefore the value of the derivative of $O_{124}$ with respect to $(1 / U)$ as $U \rightarrow \infty$ must be identically zero for these cases.

It is important to emphasize that the property 2) above is probably a consequence of the finite cluster Hamiltonian, and in all probability does not survive for arbitrary Hamiltonians in the themodynamic limit.

The specific value $t=t / 2$ is singular. The derivative mentioned above is zero if the limit $U \rightarrow \infty$ is taken before $t \rightarrow t / 2$; the slope is finite if the limits are taken in the opposite order (see Fig. 9). In the latter case condition 3) is violated (the ground 
state of the Hamiltonian is not an eigenstate of $\mathbf{S}_{1} \cdot \mathbf{S}_{2} \times \mathbf{S}_{4}$ ).

There are three points where the ground state may be adiabatically continued (at fixed $r^{\prime}(t)$ from $U=0^{+}$to $U \rightarrow \infty$ without any level crossings: at $t=0$; at $l=t / 2$; and at $l=0$. The two cases when one of the hopping parameters vanishes produce the smooth crossover from a flux-phase spin-density-wave limit $\left(U=0^{+}\right)$to a quantum Neel limit $(U \rightarrow \infty)$ as suggested ${ }^{47}$ by Hsu. The other case $\left(t^{\prime}=t / 2\right)$ indicates that the $U=0$ ground state of a tight-binding model can be smoothly related to the ground state of a frustrated Heisenberg model with no intervening phase transition.

On a $4 \times 4$ cluster, ${ }^{13}$ the ground state of the fustrated Heisenberg model remains nondegenerate, although there is a sharp level repulsion in the vicinity of the transition between the two- to four-sublattice Neel states. There is as yet no evidence which points to the existence of a "spin-liquid" phase in any finite system calculation.

There are a few interesting results for the excited states in the chiral Hubbard model. The particle-hole parity operator is not an independent quantity, but rather satisfies $R=(-1)^{S+J}$ for all cases tested. A few accidental degeneracies remain in the many-body spectrum: Fifteen cases arise from many-body eigenstates that are simultaneous eigenvectors ${ }^{48}$ of the kinetic energy and potential energy operators of (4.2); and eight levels of ${ }^{1} \gamma_{3}(J=0)$ symmetry are degenerate with eight levels of ${ }^{1} \gamma_{4}(J=0)$ symmetry. Heilmann's method ${ }^{45}$ is used to show that the latter degeneracies do not correspond to any pararneter-independent symmetries, so they probably arise from the dynamical effect ${ }^{49}$ discussed in Ref. 33.

\section{IV.4 Conclusions}

Exact solutions of the Hubbard model on an eight-site square lattice cluster with nearest- and next-nearest-neighbor hopping $t$ and $t$ have been presented for two different flux distributions. In the first case (the "ordinary" Hubbard model), the flux through any closed loop vanishes, and all link phases $\phi_{i j}$ can be set to zero. In the 
seconu case (the "chiral" Hubbard model), the link phases are selected so that the flux through every elementary triangle is $\pi / 2$. The ground and low-lying states of an eight site cluster with PBC are exactly solved for both the ordinary Hubbard model (Fig. 4.1) with nearest- and next-nearest-neighbor hopping and the chiral Hubbard model (Fig. 4.2) with nearest- and next-nearest-neighbor hopping in the presence of a "magnetic field" which couples only to orbital motion and whose strength corresponds to one-half flux quantum per plaquette. These exact solutions are made possible by using the cluster-symmetry group of the models and spin-rotation symmetry. In the case of the ordinary Hubbard model, the cluster-symmetry group includes the space group and extra site-permutation operators (which are a finite-size effect of the eight-site squarelattice cluster). In the case of the chiral Hubbard model, the complete clustersymmetry group is composed of combinations of gauge transformations and spacegroup operations.

The phase diagram of the half-filled ordinary Hubbard model (with zero flux through every closed path) is shown in Fig. 4.3. For small or large $t / t$, the ground state of the system is seen to vary smoothly from the $U=0^{+}$spin-density-wave limit to the large- $U$ quantum Néel limit, as discussed ${ }^{9}$ by Schrieffer, Wen, and Zhang. When both hopping parameters are comparable, however, we find several level crossings between the small and large- $U$ limits, and a complicated set of ground-state phases at small and intermediate $U$. These intermediate- $U$ phases include a peculiar state which has a nonzero (but unsaturated) magr:slik: moment, and contradicts the folklore that the ground state of a half-filled Hubbard risiel is spin quenched. It is found that for $0<t<t$ there is no path from $U=0^{+}$to large $U$ along which the ground state changes continuously. Thus when both nearest- and next-nearest-neighbor hopping contribute appreciably to the kinetic energy, one cannot apply a simple weak-coupling theory to extract the physics of the corresponding large- $U$ frustrated Heisenberg spin system. According to Fig. 4.3, the best path from weak coupling to to the frustrated 
Heisenberg model either stars with $t^{\prime}=0$, proceeds to large $U$, and then turns on a finite $t$, or starts with $t>t$, proceeds to large- $U$, and then decreases $t$ to values $t<t$.

The phase diagram of the half-filled chiral Hubbard model with a flux of $\pi / 2$ per triangle is displayed in Fig. 4.6. When $t$ or $t$ vanish, there is a smooth transit from the flux-phase spin-density-wave limit to the quantum Neel limit, as suggested ${ }^{47}$ by Hsu. When $t$ and $r$ are comparable, however, the phase diagram acquires a pleasing simplicity when compared with that of the ordinary Hubbard model shown in Fig. 4.3. A single phase at $t^{\prime}=t / 2$ stretches from the $U=0$ axis all the way to $U=\infty$, where it pinches off to a single point at the transition between the two-sublatrice and the foursublatrice Néel states. In accord with several other calculations, no evidence was found for an intermediate spin-liquid phase (except for a single point) in the spin-1/2 Heisenberg model with nearest- and next-nearest-neighbor antiferromagnetic couplings on the relatively small eight-site cluster. The results do suggest, however, that the ground state of a $U=0$ tight-binding model (at one value of $t^{\prime} / t$ ) may be smoothly related to the ground state of a frustrated Heisenberg model without an intervening phase transition. It is plausible that exact-diagonalization studies of Heisenberg models on larger clusters would indicate whether this region of analytic continuation becomes finite or disappears entirely by comparing the symmetries of (candidate) $U=0$ tight-binding ground states to the corresponding frustrated Heisenberg-model ground state.

\section{IV.5 Appendix: Full Gauge-Space Group}

The uniform gauge transformation $E$ (Eq. 4.11) is a unitary operator that corresponds to multiplication of a many-body wavefunction by the overall phase factor $\exp \left[i \pi\left(N_{\uparrow}+N_{\downarrow}\right)\right]$ which yields $1[-1]$ for an even [odd] number of electrons. The element $E$ also commutes with every element of the gauge-space group in Table 
4.7. The uniform gauge transformation, therefore, has an identical relationship to the gauge-space group (when one considers representations with an even or odd number of electrons) is a rotation by $2 \pi$ has to ordinary space groups (when one considers representations with integral or half-integral $\operatorname{spin}^{50}$ ).

The intraduction of the uniform gauge transformation as an independent group element produces a double group, called the full gauge-space group, that has 64 elements. Six different gauge transformations

$$
\begin{gathered}
\chi_{1}=G_{3} G_{7}, \chi_{2}=G_{4} G_{8}, \chi_{3}=G_{1} G_{4} G_{5} G_{8}, \chi_{4}=G_{2} G_{3} G_{6} G_{7}, \\
\chi_{5}=G_{1} G_{2} G_{3} G_{5} G_{6} G_{7}, \chi_{6}=G_{1} G_{2} G_{4} G_{5} G_{6} G_{8},
\end{gathered}
$$

are required for closure. There are 19 classes in the double group and three of those classes include barred and unbarred elements (a barred element corresponds to an unbarred element multiplied by $(E \mid 0\})$. The group elements and class strus:ture are summarized in Table 4.11. It must be reiterated that the double-group structure of the full gauge-space group is not related to the wotal spin of the electrons, but rather it arises from the transformation properties of the chiral Hubbard model under gauge transformations.

Since some of the classes of the full gauge-space group include both barred and unbarred elements, all of the "double-valued" representations are at least twofold degenerate, which is analogous to Kramers degeneracy. The eleven "single-valued" representations of the full gauge-space group (which correspond to representations with an even number of electrons) can be found in Table 4.8. Table 4.12 records the eight "double-valued" representations (which correspond to representations with an odd number of electrons) for the full gauge-space group including the compatibility relations with the real space group (Table 4.6) in the last column.

There is no Brillouin zone or even a gauge-Brillouin zone for the "double-valued" representations because the gauge-translation subgroup (composed of all elements with 
a point-group operation $E$ or $\bar{E}$ ) forms a nonabelian invariant subgroup of the full gauge-space group: there is no Bloch's theorem.

The one-electron band structure of the chiral Hubbard model is easily determined. There are four twofold degenerate levels of symmetries $\omega_{1}$ (enlerigy $\varepsilon=-\sqrt{8} t$ ), $\omega_{2}(\varepsilon=\sqrt{8} t), \omega_{5}\left(\varepsilon=4 t^{\prime}\right)$, and $\omega_{6}\left(\varepsilon=-4 r^{\prime}\right)$. The noninteracting ground state for the half-filled band consists of the filled shells of the $\omega_{1}$ and $\omega_{6}$ levels, has symmetry ${ }^{1} \gamma_{2}$, and is nondegenerate (whenever $r$ and $r$ are both nonzero).

The fact that all representations corresponding to an odd number of electrons are twofold degenerate implies that a symmetry-lowering distorion of the phases in (4.2), as has been recently proposed ${ }^{10}$ for the spinons and holons, would be energetically more favorable than the "uniform" choice of the chiral Hubbard model. 


\section{IV.6 References for Chapter IV}

1 P.W. Anderson, Science 235, 1196 (198\%).

2 S. Kivelson, D.S. Rokhsar, and J.P. Sethna, Phys. Rev. B 35, 8865 (1987).

3 V. Kalmeyer and R.B. Laughlin, Phys. Rev. Lett. 59, 2095 (1987); R.B. Laughlin, Science 242, 525 (1988).

4 I. Affleck and J.B. Marston, Phys. Rev. B 37, 3774 (1988); J.B. Marston and I. Affleck, ibid. B 39, 11538 (1989).

5 G. Baskaran and P.W. Anderson, Phys. Rev. B 37, 580 (1989).

6 D.S. Rokhsar, Phys. Rev. B 42, 2526 (1990).

7 C. Gros, Phys. Rev, B 38, 931 (1988).

8 X.G. Wen, F. Wilczek, and A. Zee, Phys. Rev, B 39, 11413 (1989).

9 J.R. Schrieffer, X.G. Wen and S.-C. Zhang, Phys. Rev. B 39, 11663 (1988).

10 D.S. Rokhsar, Phys. Rev. Lett. 65, 1506 (1990).

11 A Néel antiferromagnet is the quantum-mechanical ground state of an unfrustrated Heisenberg model and can be viewed as a classical Neel state with quantum fluctuations.

Numerical studies of frustrated spin-1/2 Heisenberg models suggest that Néel order is destroyed for sufficient frustration, but the nature of the ground states remains unclear. See, e.g., E. Dagotto and A. Moreo, Phys. Rev. B 39, 4744 (1989) and Ref. 13.

13 F. Figuerido, A. Karlhede, S. Kivelson, S. Sondhi, M. Rocek, and D.S. Rokhsar, Phys. Rev. B 41, 4619 (1990).

14 For a review see L.M. Falicov in Recent Progress in Many-Body Theories Vol. 1, edited by A.J. Kaltio, E. Pajanne, and R.F. Bishop, (Plenum, New York, 1988) p. 275; J. Callaway, Physica B 149, 17 (1988). 
L.M. Falicov, Group Theory and Its Physical Applicarions, (University of Chicago, Chicago, 1966) p. $144 f f$.

D. Pines, The Many-Bady Problem (Benjamin/Cummings, Reading, 1962).

A.B. Harris and R.V. Lange, Phys. Rev. 157, 295 (1967).

L.M. Falicov and R.A. Harris, J. Chem. Phys. 51, 3153 (1969).

A.M. Oles, B. Oles, and K.A. Chao, J. Phys. C 13, L979 (1980); J. Rß̈ssler, B. Fernandez, and M. Kiwi, Phys. Rev. B 24, 5299 (1981); D.J. Newman, K.S. Chan, and B. Ng, J. Phys. Chem. Solids 45, 643 (1984); L.M. Falicov and R.H. Victora, Phys. Rev. B 30, 1695 (1984); C.M. Willet and W.-H. Steeb, J. Phys. Soc. Japan 59, 393 (1990).

E. Kaxiras and E. Manousakis, Phys. Rev. B 37, 656 (1988); E. Kaxiras and E. Manousakis, ibid., 38, 866 (1988); J. Bonca, P. Prelovsek, and I. Sega, ibid., 39, 7074 (1989); J.A. Riera and A.P. Young, ibid., 39, 9697 (1989); ibid., 40, 5285 (1989); E. Dagotto, A. Moreo, and T. Bames, ibid., 40, 6721 (1989); M. Ogata and H. Shiba, J. Phys. Soc. Japan 58, 2836, (1989); Y. Fang, A.E. Ruckenstein, E. Dagotto, and S. Schmitt-Rink, Phys. Rev. B 40, 7406 (1989); Y. Hasegawa and D. Poilblanc, ibid., 40, 9035 (1989); Int. J, Mod. Phys. B 3, 1853 (1989); A. Parola, S. Sorella, S. Baroni, M. Parrinello, and E. Tosatt, ibid., 3, 139 (1989); M. Imada and Y. Hatsugai, J. Phys. Soc. Japan 58, 3752 (1989); D.M. Frenkel, R.J. Gooding, B.I. Shraiman, and E.D. Siggia, ibid., 41, 350 (1990); E. Dagotto, A. Moreo, R.L. Sugar, and D. Toussaint, Phys. Rev. B 41, 811 (1990); D. Poilblanc, Y. Hasegawa, and T.M. Rice, ibid., 41, 1949 (1990); E. Dagoto, A. Morco, R. Joynt, S. Bacci, and E. Gagliano, ibid., 41, 2585 (1990); K.-H. Luk and D.L. Cox, ibid., 41, 4456 (1990); V. Elser, D.A. Huse, B.I. Shraiman, and E.D. Siggia, ibid., 41, 6715 (1990); D. Poilblanc and Y. Hasegawa, ibid., 41, 6989 (1990); J. Richter, Z. Phys. B 79, 403 (1990); I. Sega and P. Prelovsek, Phys. Rev. B 42, 892 (1990); T. Itoh, M. Arai, and T. Fujiwara, ibid., 42, 4834 
(1990); D. Poilblanc and E. Dagotto ibid., 42, 4861 (1990); A. Moreo and E. Dagotto, ibid., 42, 4786 (1990); E. Gagliano, S. Bacci, and E. Dagotto, ibid., 42, 6222 (1990); G. Fano, F. Ortolani, and A. Parola, ibid., 42, 6877 (1990).

21 J.K. Freericks and L.M. Falicov, Phys. Rev. B 42, 4960 (1990).

22 J. Hubbard, Proc. R. Soc. London, Ser. A 276, 238 (1963); ibid., 277, 237 (1964); ibid., 281, 401 (1964); ibid., 285, 542 (1965); ibid., 296, 82 (1967); ibid., 296, 100 (1967).

23 C.J. Heilmann and E.H. Iieb, Trans. NY Acad. Sci. 33, 116 (1971).

24 C.N. Yang, Phys. Rev. Lett. 63, 2144 (1989); M. Pernici, Europhys. Leit. 12, 75 (1989); S. Zhang, Phys. Rev. Lett. 65, 120 (1990).

C.N. Yang and S.C. Zhang, Mod. Phys. Lett. A 4, 759 (1990).

L.P Bouckaert, R. Smoluchowski, and E. Wigner, Phys. Rev. 50, 58 (1936).

R. Saito, Sol. St. Commun. 72, 517 (1989); T. Ishino, R. Saito, and H. Kamimura, J. Phys. Soc. Japan, 59, 3886 (1990); see a so Chapter V.

I.M. Falicov, Group Theory and Its Physical Applications, (University of Chigago Press, Chicago, 1966) p. $155 f$.

C. Kittel, Quansum Theory of Solids, (Wiley, New York, 1987) pp. 179ff.

The labels of the many-body eigenstates are called gauge-wavevectors because they determine the phase shift of these states under normal and gaugetranslations.

S. Kivelson and W.-K. Wu, Phys. Rev. B 34, 5423 (1986).

W. Brinkman and T.M. Rice, Phys. Rev. B 2, 1324 (1970); B.S. Shastry, J. Stat. Phys. 50, 57 (1988).

H. Grosse, Lett. Math. Phys. 18, 151 (1989). 
$\therefore$ M. Tinkham, Group Theory and Quantum Mechanics, McGraw-Hill, New York, 1964) p. 20ff.; p. $80 \mathrm{ff}$.

L.M. Falicov, Group Theory and Its Physical Applications, (University of Chicago, Chicago, 1966) p. 20ff.; p. 46ff.

A.W. Luehrmann, Adv. in Phys. 17, 1 (1968).

In the case of representations that have complex-valued $(1,1)$ matrix elements the projection is onto the subspace spanned by the complex representation and its conjugate so that all marrix elements are real.

R.P. Brent, ACM Trans. on Math. Soft. 4, 57 (1978).

J.H. Wilkinson and C. Reinsch, Handbook for Automatic Computation, Vol. II: Linear Algebra, ed. FL. Bauer, (Springer-Verlag, New York, 1971) p. 227.

J. Cullum and R.A. Willoughby, Lanczos Algorithms for Large Symmetric Eigenvalue Computations Vol. 1 and 2 (Birkhauser, Basel, 1985).

41 E.H. Lieb and F.Y. Wu, Phys. Rev. Lett. 20, 1445 (1968).

P.W. Anderson, Solid State Phys. 14, 99 (1969); J.R. Schrieffer and P.A. Wolff, Phys. Rev. 149, 491 (1966); A.H. MacDonald, S.M. Girvin, and D. Yoshioka, Phys. Rev. B 37, 9753 (1988); A.M. Oles, ibid., 41, 2562 (1990); A.H. MacDonald, S.M. Girvin, and D. Yoshioka, ibid., 41, 2565 (1990).

44 Two cases have nontrivial energy eigenvalues: nine of the ${ }^{5} \Gamma_{1 n}$ levels are degenerate for all values of the interaction strength with nine of the ${ }^{5} M_{1 n}$ levels; and nine of the ${ }^{3} \Gamma_{l n}$ levels are degenerate with nine of the ${ }^{3} M_{1 n}$ levels. Three cases arise from many-body states that are annihilated by the kinetic energy operator (i.z., they are simultaneous eigenstates of the kinetic and potential energy operators): the level $E=U$ has a degeneracy $\mathrm{d}=129 ; E=2 U$ has $\mathrm{d}=107$; and $E=3 U$ has $\mathrm{d}=6$. 
45 This method uses the fact that the $U$-independent invariant subspaces of the Hamiltonian correspond to the invariant subspaces of a test matrix that is constructed out of the Hamiltonian (with $U=1$ ) and is expressed in a coordinate basis that diagonalizes the Hamiltonian for a particular value of $U \neq 1$. For further details see OJ. Heilmann, J. Math. Phys. 11, 3317 (1970).

The invariant subspace decomposition produced the trivial one-dimensional suispaces that correspond to each eigenvector that is a simultaneous eigenvector of the two terms in (4.2) and it produced thirteen nontrivial decompositions in the subspaces highlighted in bold in Table 4.10. The subspace ${ }^{3} M_{1 n}$ decomposes into three nontrivial invariani subspaces and the other twelve decompose into two nontrivial invariant subspaces. It is easy to show by a counting argument that the extra symmetry is not a permutation symmetry of the eight sites. There are probably two possibilities that can explain the extra symmetry: there may be an extra permutation symmetry of the sixteen orbitals that conserves $S^{2}$ and $S_{z}$ but mixes the space and spin labels creating an even larger group that would explain the accidental degeneracies; there may be an operator similar to the pseudospin $J^{2}$ that causes the decomposition and the accidental degeneracies are explained by a dynamical effect using conserved currents as in Ref. 33.

T. Hsu, Phys. Rev. B 41, 11379, 1990.

48 A larger number of parameter-independent eigenstates than those constructed in Ref. 25 [out of states that have maximal values of pseudospin $(J=M / 2-S=4-S)$ ) have been found (816 found versus 640 predicted); this disproves Yang and Zhang's conjecture that the only parameter-independent eigenstates are those lying in a maximal-pseudospin family.

49 The parameter-dependent conserved current would commute with $\left\{E \mid \tau_{2}\right)$, $\chi_{3}\left\{E \mid \tau_{4}\right\}$, and $R$; anticommute with $\chi_{1}\left(C_{4} \mid 0\right\}$; and commute or anticommute with both $S U$ (2) operators. It would take each of the eight eigenstates with ${ }^{1} \gamma_{3}$ 
$(J=0)$ symmetry and map them into the corresponding eigenstate with ${ }^{1} \gamma_{4}(J=0)$ symmetry that has the same energy eigenvalue (and vice versa). The other many-body eigenstates would either be annihilared by the current or, if the state is a member of a multiplet (i.e., has symmetry $x_{1}, x_{2}$, or $\sigma_{1}$ ), it may be mapped into another member of the multiplet by the current.

50 L.M. Falicov, Group Theory and Its Physical Applications, (University of Chicago, Chicago, 1966) p. $103 \mathrm{ff}$. 


\section{IV.7 Tables for Chapter IV}

Table 4.1. Renormalized hopping matrix elements $t_{i j}$ for $(i<j)$ in the ordinary Hubbard model. The eight cluster sites are illustrated in Fig. 4.1. All diagonal matrix elements $t_{i i}$ are zero and the matrix elements $t_{i j}$ with $i>j$ are determined by hermiticity $\left(t_{i j}=t_{j i}^{*}\right)$.

\begin{tabular}{cllll}
\hline \hline parameter & \multicolumn{4}{c}{ indices $(i j)$} \\
\hline$-t$ & $(12)$ & $(14)$ & $(16)$ & $(18)$ \\
& $(23)$ & $(25)$ & $(27)$ & $(34)$ \\
& $(36)$ & $(38)$ & $(45)$ & $(47)$ \\
& $(56)$ & $(58)$ & $(67)$ & $(78)$ \\
\hline$-2 t^{\prime}$ & $(13)$ & $(17)$ & $(24)$ & $(28)$ \\
& $(35)$ & $(46)$ & $(57)$ & $(68)$ \\
\hline 0 & $(15)$ & $(26)$ & $(37)$ & $(48)$ \\
\hline \hline
\end{tabular}


Table 4.2. Renormalized hopping matrix elements $t_{i j}$ for $(i<j)$ in the chiral Hubbard model. The eight cluster sites are illustrated in Fig. 4.2. All diagonal matrix elements $t_{i i}$ are zero and the matrix elements $t_{i j}$ with $i>j$ are determined by hermiticity $\left(t_{i j}=t_{j i}^{*}\right)$.

\begin{tabular}{ccccc}
\hline \hline parameter & \multicolumn{4}{c}{ indices $(i j)$} \\
\hline$-t$ & $(12)$ & $(14)$ & $(16)$ & $(18)$ \\
& $(23)$ & $(25)$ & $(27)$ & $(36)$ \\
& $(45)$ & $(56)$ & $(58)$ & $(67)$ \\
\hline$t$ & $(34)$ & $(38)$ & $(47)$ & $(78)$ \\
\hline$-2 i r$ & $(17)$ & $(28)$ & & \\
\hline $2 i r$ & $(13)$ & $(24)$ & $(35)$ & $(46)$ \\
& $(57)$ & $(68)$ & & \\
\hline 0 & $(15)$ & $(26)$ & $(37)$ & $(48)$ \\
\hline \hline
\end{tabular}


Table 4.3. Character table for the space group of the eight-site square-lattice cluster (ordinary Hubbard model). The symbol $E$ is the identity, $C_{n}^{m}$ is the rotation by $2 \pi m / n$ about the $z$-axis, $\sigma$ denotes the mirror planes perpendicular to the $x-$ and $y$-axes and $\sigma^{\prime}$ denotes the mirror planes perpendicular to the diagonals $x \pm y$. The translations are denoted by 0 (no translation), $\tau$ (nearest-neighbor translation), $\theta$ (next-nearestneighbor), and $\Omega$ (third-nearest-neighbor). The subscripts $\|$ and $\perp$ refer to translations parallel to or perpendicular to the normals of the mirror planes.

\begin{tabular}{|c|c|c|c|c|c|c|c|c|c|c|c|c|c|c|c|c|}
\hline & $\begin{array}{l}1 \\
E \\
0\end{array}$ & $\begin{array}{c}8 \\
c_{4} \\
0 \theta \Omega\end{array}$ & $\begin{array}{c}2 \\
c_{4}^{2} \\
0 \Omega\end{array}$ & $\begin{array}{c}4 \\
0 \\
0 \Omega\end{array}$ & $\begin{array}{c}4 \\
\sigma \\
0 \theta_{1}\end{array}$ & $\begin{array}{c}4 \\
E \\
\tau\end{array}$ & $\begin{array}{c}B \\
C_{4} \\
\tau\end{array}$ & $\begin{array}{r}4 \\
C_{4}^{2} \\
t\end{array}$ & $\begin{array}{l}4 \\
\sigma \\
\tau_{H}\end{array}$ & $\begin{array}{l}4 \\
0 \\
\tau_{1}\end{array}$ & $\begin{array}{l}8 \\
\sigma \\
7\end{array}$ & $\begin{array}{l}2 \\
E \\
\theta\end{array}$ & $\begin{array}{c}2 \\
c_{1}^{2} \\
\theta\end{array}$ & $\begin{array}{l}4 \\
\sigma \\
\theta\end{array}$ & $\begin{array}{c}4 \\
\sigma \\
\theta_{\|} \Omega\end{array}$ & $\begin{array}{l}1 \\
E \\
\Omega\end{array}$ \\
\hline$\Gamma_{1}$ & 1 & 1 & 1 & 1 & 1 & 1 & 1 & 1 & 1 & 1 & 1 & 1 & 1 & 1 & 1 & 1 \\
\hline$\Gamma_{2}$ & 1 & 1 & 1 & -1 & -1 & 1 & 1 & 1 & -1 & -1 & -1 & 1 & 1 & -1 & -1 & 1 \\
\hline$\Gamma_{3}$ & 1 & -1 & $i$ & 1 & -1 & 1 & -1 & 1 & 1 & 1 & -1 & 1 & 1 & 1 & -1 & 1 \\
\hline$r_{4}$ & 1 & -I & $\mathbf{I}$ & .1 & 1 & 1 & -1 & 1 & -1 & $-I$ & 1 & 1 & 1 & -1 & 1 & 1 \\
\hline$\Gamma_{s}$ & 2 & 0 & -2 & 0 & 0 & 2 & 0 & -2 & 0 & 0 & 0 & 2 & -2 & 0 & 0 & 2 \\
\hline$M_{1}$ & 1 & 1 & 1 & 1 & 1 & -1 & -1 & -1 & -1 & -1 & -1 & 1 & 1 & 1 & 1 & 1 \\
\hline$M_{2}$ & 1 & 1 & 1 & -1 & -1 & -1 & -1 & -1 & 1 & 1 & 1 & 1 & 1 & -1 & -1 & 1 \\
\hline$M_{3}$ & 1 & -1 & 1 & 1 & -1 & -1 & $\mathbf{I}$ & -1 & .1 & -1 & 1 & 1 & 1 & 1 & -1 & 1 \\
\hline$M_{4}$ & 1 & -1 & 1 & -1 & 1 & -2 & 1 & -1 & 1 & 1 & -1 & 2 & 1 & -1 & 1 & 1 \\
\hline$M_{s}$ & 2 & 0 & .2 & 0 & 0 & -2 & 0 & 2 & 0 & 0 & 0 & 2 & -2 & 0 & 0 & 2 \\
\hline$x_{1}$ & 2 & 0 & 2 & 2 & 0 & 0 & 0 & 0 & 0 & 0 & 0 & .2 & -2 & -2 & 0 & 2 \\
\hline$x_{2}$ & 2 & o & 2 & -2 & 0 & 0 & 0 & 0 & 0 & 0 & 0 & -2 & -2 & 2 & 0 & 2 \\
\hline$x_{3}$ & 2 & 0 & -2 & 0 & 0 & o & 0 & 0 & -2 & 2 & 0 & -2 & 2 & 0 & 0 & 2 \\
\hline$x_{4}$ & 2 & 0 & -2 & 0 & 0 & 0 & 0 & 0 & 2 & -2 & 0 & -2 & 2 & 0 & 0 & 2 \\
\hline$\Sigma_{j}$ & 4 & 0 & 0) & 0 & 2 & 0 & 0 & 0 & 0 & 0 & 0 & 0 & 0 & 0 & -2 & -4 \\
\hline$\Sigma_{2}$ & 4 & 0 & 0 & 0 & -2 & 0 & 0 & 0 & 0 & 0 & 0 & 0 & 0 & 0 & 2 & .4 \\
\hline
\end{tabular}


Table 4.4. Class structure and group elements of the 128 element cluster-permutation group of the ordinary Hubbard model. The element $\boldsymbol{P}$ corresponds to the transposition of site-1 and site-5. The notation is identical to that of Table 4.3.

\begin{tabular}{|c|c|c|c|c|}
\hline class & & group elements & & size of class \\
\hline 1 & $\{E \mid 0\}$ & & & 1 \\
\hline 2 & $\left\{C_{4} \mid 0, \theta, \Omega\right\}$ & & & 8 \\
\hline 3 & $\left\{C_{4}^{2} 10, \Omega\right\}$ & & & 2 \\
\hline 4 & $\{\sigma \mid 0, \Omega\}$ & & & 4 \\
\hline 5 & $\left\{\sigma^{\prime} \mid 0, \theta_{\perp}\right\}$ & & & 4 \\
\hline 6 & {$[E \mid \tau]$} & $\left\{\sigma \mid \tau_{1}\right\}$ & & 8 \\
\hline 7 & $\left\{C_{4} \mid \tau\right\}$ & $\left\{\sigma^{\prime} \mid \bar{\tau}\right\}$ & & 16 \\
\hline 8 & $\left\{C_{4}^{2} \mid \tau\right\}$ & {$\left[\sigma \mid \tau_{\|}\right]$} & & 8 \\
\hline 9 & $\{E \mid \theta\}$ & $\left\{C_{4}^{2} \mid \theta\right\}$ & & 4 \\
\hline 10 & $\{\sigma \mid \theta\}$ & & & 4 \\
\hline 11 & $\left\{\sigma \mid \theta_{\|}, \Omega\right\}$ & & & 4 \\
\hline 12 & $\{E \mid \Omega\}$ & & & 1 \\
\hline 13 & $P\{E \mid 0\}$ & $P\left\{C_{4}^{2} \mid \Omega\right\}$, & $P\{\sigma \mid \Omega\}$ & 4 \\
\hline 14 & $P\left\{C_{4} \mid 0\right\}$ & $P\left(\sigma^{\prime} \mid \theta_{\|}\right\}$ & & 4 \\
\hline 15 & $P\left\{C_{4}^{2} 10\right\}$ & $P\{\sigma \mid 0\}$ & $P\{E \mid \Omega\}$ & 4 \\
\hline 16 & $P\left(\sigma^{\prime} \mid 0, \Omega\right\}$ & $P\left\{C_{4} \mid \theta\right\}$ & & 8 \\
\hline 17 & $P\{E \mid \tau\}$ & $P\left(C_{4}^{2} \mid \tau\right)$ & $P(\sigma \mid \tau)$ & 16 \\
\hline 18 & $P\left\{C_{4} \mid \tau\right\}$, & $P\left(\sigma^{\prime} \mid \tau\right)$ & & 16 \\
\hline 19 & $P\{E \mid \theta\}$ & $P\left\{C_{4}^{2} \mid \theta\right\}$ & $P\{\sigma \mid \theta\}$ & 8 \\
\hline 20 & $P\left\{C_{4} \mid \Omega\right\}$ & $P\left\{\sigma^{\prime} \mid \theta_{1}\right\}$ & & 4 \\
\hline
\end{tabular}


Table 4.5. Character table of the 128 element cluster-permutation group for the ordinary Hubbard model. The class structure and group elements are given in Table 4.4. The classes are labeled by their number to save space in the table below. The last column gives the compatibility relations with the irreducible representations of the real space group (Table 4.3). The subscripts $p, z$, and $n$ denote representations that have a poitive character, zero character, or negative character, respectively, for the element $P(E \mid 0\}$. The symbol $\phi$ is tised to denote representations that mix different wavevectors. 


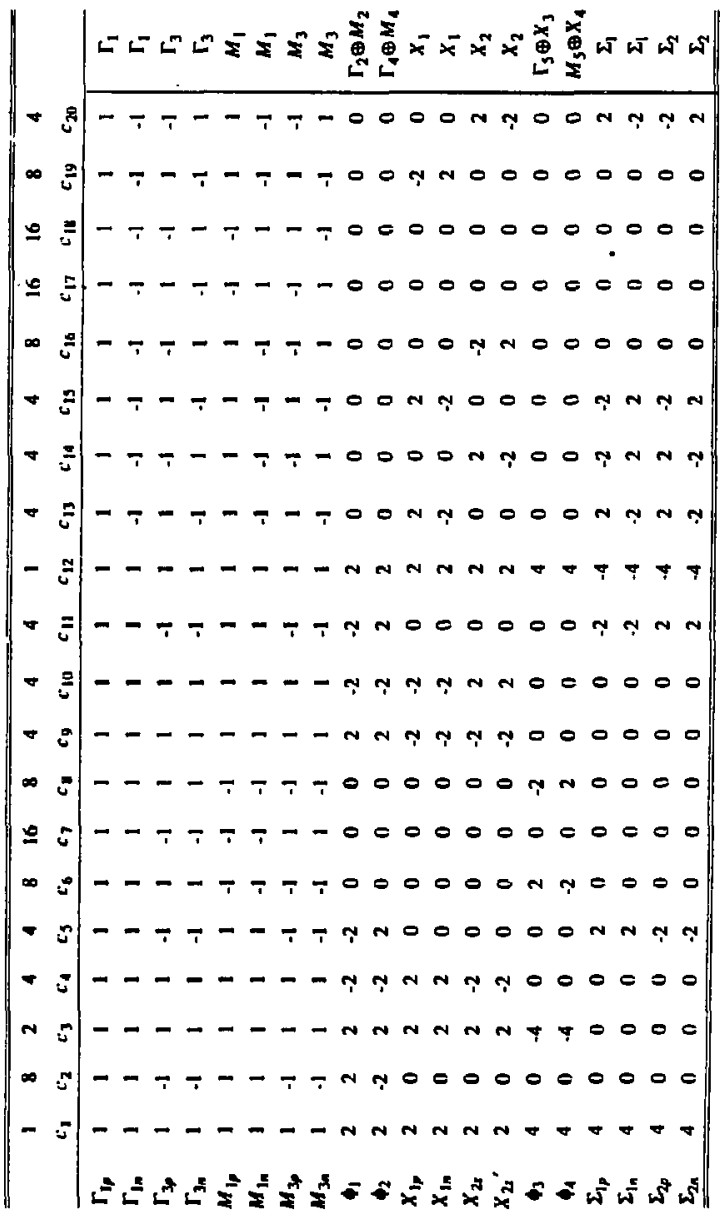


Table 4.6. Character table for the space group of the eight-site rectangular-lattice cluster (chiral Hubbard model). The notation for the space group operations is the same as in Table 4.3.

\begin{tabular}{lccccc}
\hline & 1 & 2 & 2 & 2 & 1 \\
& $E$ & $C_{2}$ & $E$ & $C_{2}$ & $E$ \\
0 & $0 \Omega$ & $\tau$ & $\tau$ & $\Omega$ \\
\multicolumn{1}{c}{} & & & & & \\
\hline$\Gamma_{1}$ & 1 & 1 & 1 & 1 & 1 \\
$\Gamma_{2}$ & 1 & -1 & 1 & -1 & 1 \\
$X_{1}$ & 1 & 1 & -1 & -1 & 1 \\
$X_{2}$ & 1 & -1 & -1 & 1 & 1 \\
$\Sigma_{1}$ & 2 & 0 & 0 & 0 & -2 \\
\hline \hline
\end{tabular}


Table 4.7. Class structure and group elements of the 32 element gauge-space group of the chiral Hubbard model (for an even number of electrons). The gauge factors $\chi_{i}$ are recorded in (4.12). The group elements without any gauge factors form a subgroup corresponding to the space group of Table 4.6.

\begin{tabular}{|c|c|c|c|}
\hline class & \multicolumn{2}{|c|}{ group elements } & size of class \\
\hline 1 & \multicolumn{2}{|c|}{$E \mid 0$} & 1 \\
\hline 2 & \multicolumn{2}{|c|}{$C_{4}^{2} \mid 0, \Omega$} & 2 \\
\hline 3 & $\chi_{1}\left\{C_{4} \mid 0, \Omega\right\}$ & $\chi_{2}\left\{C_{4} \mid \theta_{3}, \theta_{7}\right\}$ & 4 \\
\hline 4 & $\chi_{1}\left(C_{4}^{3} \mid 0, \Omega\right\}$ & $\chi_{2}\left\{C_{4}^{3} \mid \theta_{3}, \theta_{7}\right\}$ & 4 \\
\hline$s$ & $\left\{E \mid \tau_{2}, \tau_{6}\right\}$ & $\chi_{3}\left\{E \mid \tau_{4}, \tau_{8}\right\}$ & 4 \\
\hline 6 & $\left\{C_{4}^{2} \mid \tau_{2}, \tau_{6}\right\}$ & $\chi_{3}\left(C_{4}^{2} \mid \tau_{4}, \tau_{8}\right)$ & 4 \\
\hline 7 & $x_{2}\left[C_{4} \mid \tau_{2}, \tau_{6}\right]$ & $\chi_{1}\left\{C_{4} \mid \tau_{4}, \tau_{8}\right\}$ & 4 \\
\hline 8 & $\chi_{2}\left(C_{4}^{3} \mid \tau_{2}, \tau_{6}\right)$ & $\chi_{1}\left[C_{4}^{3} \mid \tau_{4}, \tau_{8}\right]$ & 4 \\
\hline 9 & \multicolumn{2}{|c|}{$\chi_{3}\left\{E \mid \theta_{3}, \theta_{7}\right\}$} & 2 \\
\hline 10 & \multicolumn{2}{|c|}{$\chi_{3}\left\{C_{4}^{2}, \theta_{3}, \theta_{7}\right\}$} & 2 \\
\hline 11 & \multicolumn{2}{|c|}{$[E \mid \Omega\}$} & 1 \\
\hline
\end{tabular}


Table 4.8. Character table of the 32 element gauge-space group for the even-electronnumber sector of the chiral Hubbard model. The class structure and group elements are given in Table 4.7. The gauge factors have been suppressed to save space in the table below. The last column gives the compatibility relations with the irreducible representations of the real space group (Table 4.6).

\begin{tabular}{ccccccccccc}
\hline 1 & 2 & 4 & 4 & 4 & 4 & 4 & 4 & 2 & 2 & 1 \\
$E$ & $C_{4}^{2}$ & $C_{4}$ & $C_{4}^{3}$ & $E$ & $C_{4}^{2}$ & $C_{4}$ & $C_{4}^{3}$ & $E$ & $C_{4}^{2}$ & $E$ \\
0 & $0 \Omega$ & $0 \theta \Omega$ & $0 \theta \Omega$ & $\tau$ & $\tau$ & $\tau$ & $\tau$ & $\theta$ & $\theta$ & $\Omega$
\end{tabular}

\begin{tabular}{l|ccccccccccc|c}
\hline$\gamma_{1}$ & 1 & 1 & 1 & 1 & 1 & 1 & 1 & 1 & 1 & 1 & 1 & $\Gamma_{1}$ \\
$\gamma_{2}$ & 1 & 1 & -1 & -1 & 1 & 1 & -1 & -1 & 1 & 1 & 1 & $\Gamma_{1}$ \\
$\gamma_{3}$ & 1 & -1 & $i$ & $-i$ & 1 & -1 & $i$ & $-i$ & 1 & -1 & 1 & $\Gamma_{2}$ \\
$\gamma_{4}$ & 1 & -1 & $-i$ & $i$ & 1 & -1 & $-i$ & $i$ & 1 & -1 & 1 & $\Gamma_{2}$ \\
& & & & & & & & & & & & \\
$m_{1}$ & 1 & 1 & 1 & 1 & -1 & -1 & -1 & -1 & 1 & 1 & 1 & $X_{1}$ \\
$m_{2}$ & 1 & 1 & -1 & -1 & -1 & -1 & 1 & 1 & 1 & 1 & 1 & $x_{1}$ \\
$m_{3}$ & 1 & -1 & $i$ & $-i$ & -1 & 1 & $-i$ & $i$ & 1 & -1 & 1 & $x_{2}$ \\
$m_{4}$ & 1 & -1 & $-i$ & $i$ & -1 & 1 & $i$ & $-i$ & 1 & -1 & 1 & $X_{2}$ \\
$x_{1}$ & 2 & 2 & 0 & 0 & 0 & 0 & 0 & 0 & -2 & -2 & 2 & $\Gamma_{1} \oplus X_{1}$ \\
$x_{2}$ & 2 & -2 & 0 & 0 & 0 & 0 & 0 & 0 & -2 & 2 & 2 & $\Gamma_{2} \oplus X_{2}$ \\
$\sigma_{1}$ & 4 & 0 & 0 & 0 & 0 & 0 & 0 & 0 & 0 & 0 & -4 & $\Sigma_{1} \oplus \Sigma_{1}$ \\
\hline
\end{tabular}


Table 4.9. Reduced Hamiltonian block sizes for the ordinary Hubbard model. The largest block size is ${ }^{3} \Sigma_{1 p}(78 \times 78)$. The numbers nighlighted in bold indicate blocks that are further reducible by a hidden parameter-independent symmetry. ${ }^{44-46}$

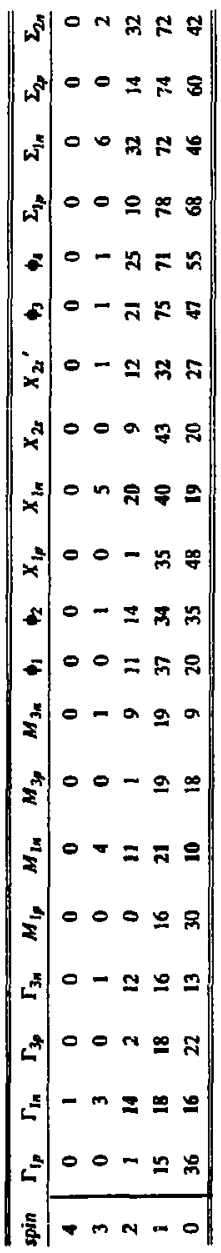


Table 4.10. Reduced Hamiltonian block sizes for the chiral Hubbard model. Note that the complex representation pairs $\left(\gamma_{3}, \gamma_{4}\right)$ and $\left(m_{3}, m_{4}\right)$ have not been separated. ${ }^{37}$ The largest block size is ${ }^{3} \sigma_{1}(296 \times 296)$.

\begin{tabular}{l|rrrrrrrrr}
\hline \hline \multicolumn{1}{l}{ spin } & $\gamma_{1}$ & $\gamma_{2}$ & $\gamma_{3}$ and $\gamma_{4}$ & $m_{1}$ & $m_{2}$ & $m_{3}$ and $m_{4}$ & $x_{1}$ & $x_{2}$ & $\sigma_{1}$ \\
\hline 4 & 1 & 0 & 0 & 0 & 0 & 0 & 0 & 0 & 0 \\
3 & 3 & 2 & 2 & 4 & 2 & 2 & 6 & 2 & 8 \\
2 & 26 & 28 & 42 & 22 & 24 & 50 & 42 & 46 & 88 \\
1 & 70 & 68 & 150 & 74 & 72 & 142 & 150 & 146 & 296 \\
0 & 72 & 70 & 94 & 50 & 62 & 110 & 114 & 102 & 216 \\
\hline \hline
\end{tabular}


Table 4.11. Class structure and group elements of the 64 element full gauge-space group of the chiral Hubbard model. The gauge factors $\chi_{i}$ are re:orded in (4.22). The barred elements correspond to the unbarred elements multiplied by $[E \mid 0]$. Classes 5 , 6, and 9 include both barred and unbarred elements.

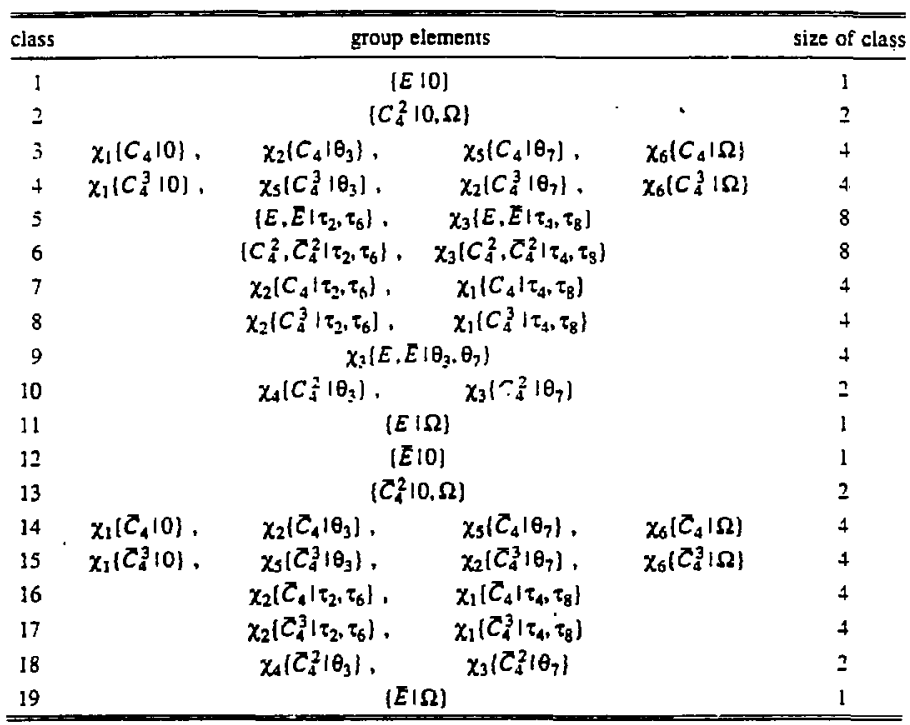


Table 4.12. Character table of the 64 element full gauge-space group for the chiral Hubbard model. The class structure and group elements are given in Table 4.11. The eleven "single-valued" representations are recorded in Table 4.8. Only the eight "double-valued" representations are recorded here. The gauge factors have been suppressed to save space in the table below. The last column gives the compatibility relations with the irreducible representations of the real space group (Table 4.6). The symbol $\alpha=(1+i) / \sqrt{2}$ is used to denote the square root of $i$.

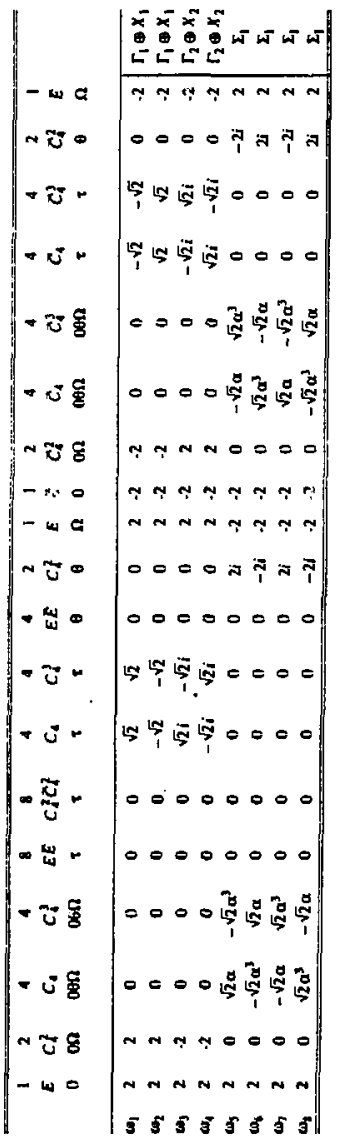




\section{T.8 Figures for Chapter IV}

Figure 4.1. Eight-site square-lattice cluster with periodic boundary conditions for the ordinary Hubbard model in (a) real and (b) reciprocal space. The nearest-neighbor hopping is indicated in (a) by thick solid lines, the next-nearest-neighbor hopping by thin dashed lines (see Table 4.1), and the primitive unit cell is highlighted in gray. Note that the four next-nearest neighbors of site 1 are two each of the sites 3 and 7 . The four symmetry stars in (b) are $\Gamma=(0,0) ; M=(1,1) \pi / a ; X=(1,0) \pi / a$; and $\Sigma=(1,1) \pi / 2 a$. 
a)

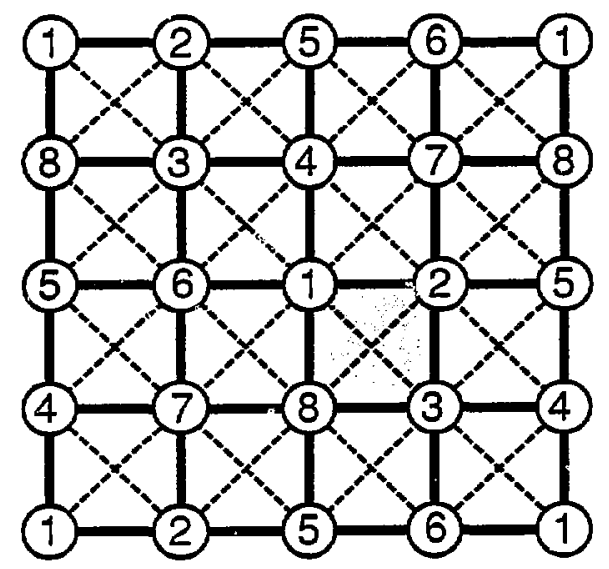

b)

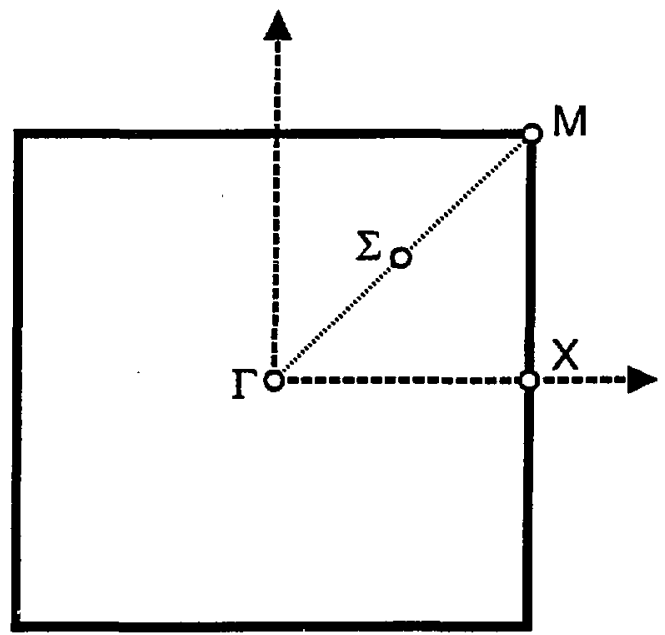


Figure 4.2. Eight-site cluster with periodic boundary conditions for the chiral Hubbard model in (a) real and (b) reciprocal space. The nearest-neighbor hopping is indicated in (a) by thick solid lines $(-t)$ and thick dotted lines $(+t)$, and the next-nearestneighbor hopping by thin dashed lines in the direction of the arrow (+it) and in the opposite direction of the arrow (-it) [see Table 4.2]. The gauge is chosen so that each elementary triangle contains a flux of $\pi / 2$, the nearest-neighbor hopping elements are real, and the next-nearest-neighbor hopping elements are imaginary. The rectangular primitive unit cell is highlighted in gray. The four wavevectors of the real space group are indicated by white dots in (b) and correspond to $\Gamma=(0,0) ; X=(1,0) \pi / a$; and $\Sigma=(1,1) \pi / 2 a,(-1,1) \pi / 2 a$. The Brillowin zcne for the chiral Hubbard model in the chosen gauge is highlighted in gray. The black dots in (b) comespond to the four additional gauge-wavevectors of the enlarged gauge-Brillouin zone for the gauge-space group of the chiral Hubbard model [see the description in (vii) of Section IV.2]. 
a)

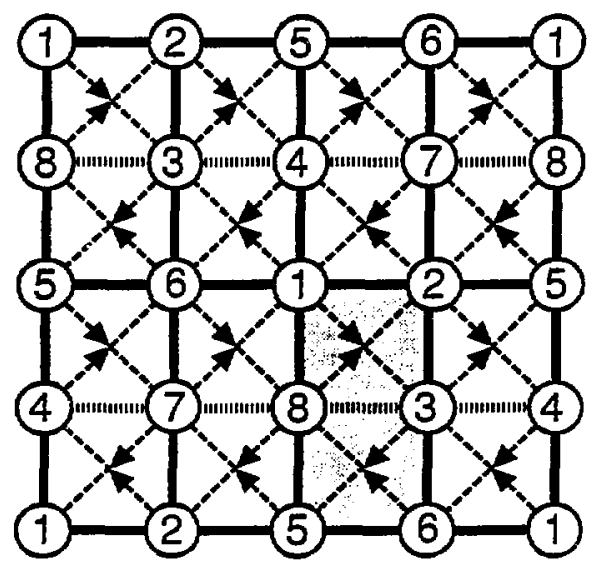

b)

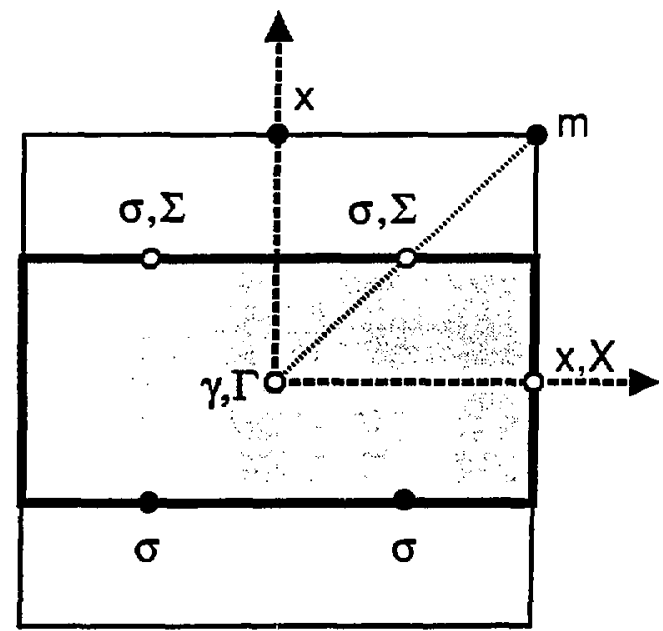


Figure 4.3. Ground-state phase diagram for the ordinary Hubbard model at half filling $(N=M=8)$. The vertical axis records the relative hopping [Eq. (4.19)] and the horizontal axis records the interaction strength [Eq. (4.20)]. The labels denote the ground-state symmetry for each corresponding phase as given in Table 4.5. The ground state is degenerate at $r^{\prime}=t / 2$ : the dashed line (at small $U$ ) corresponds to the ground state ${ }^{1} \phi_{2} \oplus^{1} \phi_{4}$ and the solid line (at large $U$ ) corresponds to the ground state ${ }^{1} \Gamma_{1 n} \oplus{ }^{1} \Gamma_{1 n} \oplus{ }^{1} \Gamma_{3 n}$. Note that the phase ${ }^{5} \Sigma_{2 n}$ is a ferrimagnetic ground state and the regions where adiabatic continuation is possible are $t^{\prime}=0$ and $t^{\prime}>t$.

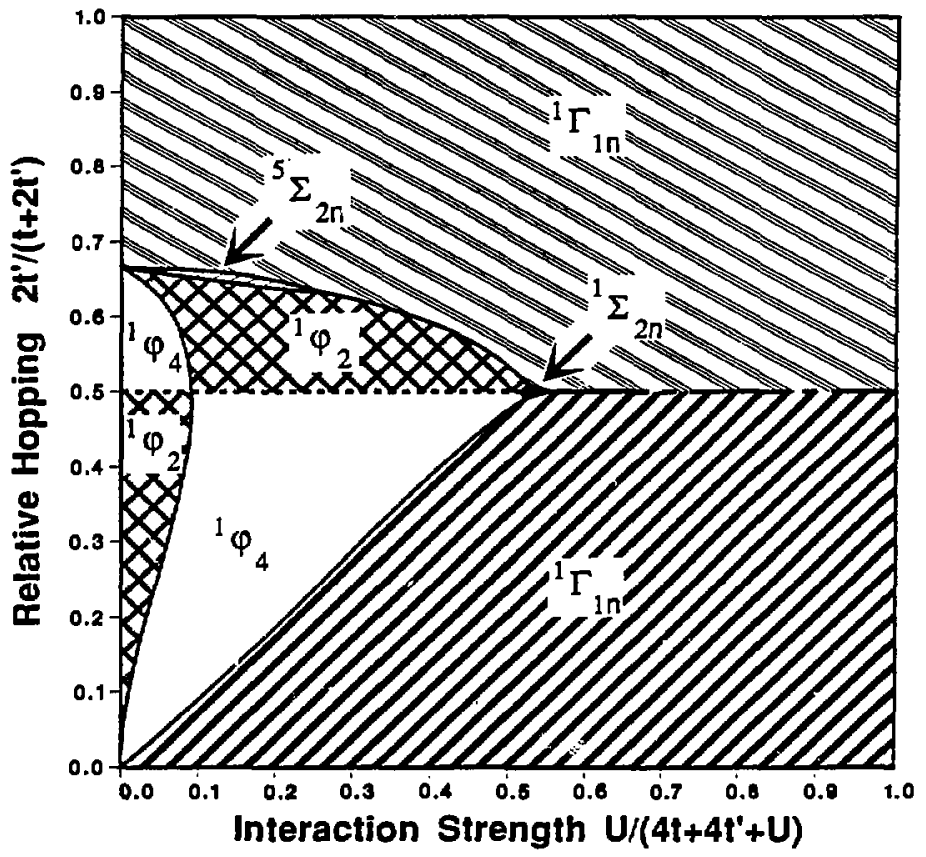


Figure 4.4. Spin-spin correlation functions $L_{i}$ [Eq. (4.16a)] for three different values of $t^{\prime} t t$ in the ordinary Hubbard model. The value of the cornelation function lies on the vertical axis and the interaction strength [Eq. (4.20)] lies on the horizontal axis. The labels 0 (on-site), 1 (nearest neighbor), 2 (next-nearest neighbor), and 3 (thirdnearest neighbor) denote the subscript $i$. Discontinuities in the spin-spin correlation functions occur only at the level crossings (see Fig. 4.3). At large $U$ the ground state is ordered as a two-sublattice Neel antiferromagnet $(t<t / 2)$ or a four-sublattice NEel antiferromagnet $\left(t^{\prime}>t / 2\right)$. Note that the case with $t^{\prime}=0.95 t$ includes the correlation functions for the magnetic phase at moderate values of $U$.

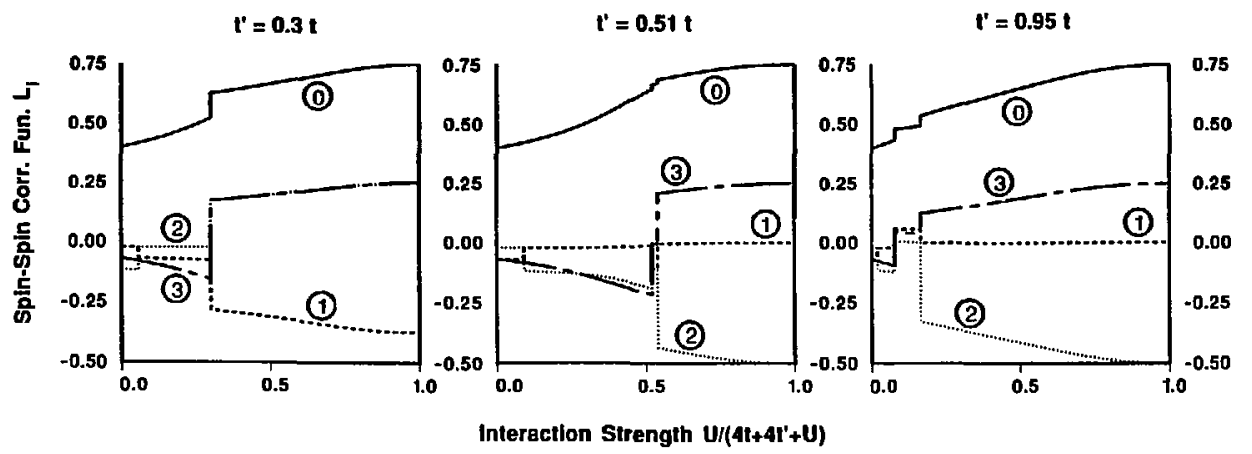


Figure 4.5. Number-number correlation functions $N_{i}$ [Eq. (4.16c)] for three different values of $t^{\prime} t t$ in the ondinary Hubbard model. The vertical axis is the interaction strength [Eq. (4.20)] and the labels denote the subscript $i$. Note that $N_{i} \rightarrow 1$ when $U \rightarrow \infty$, as expected.

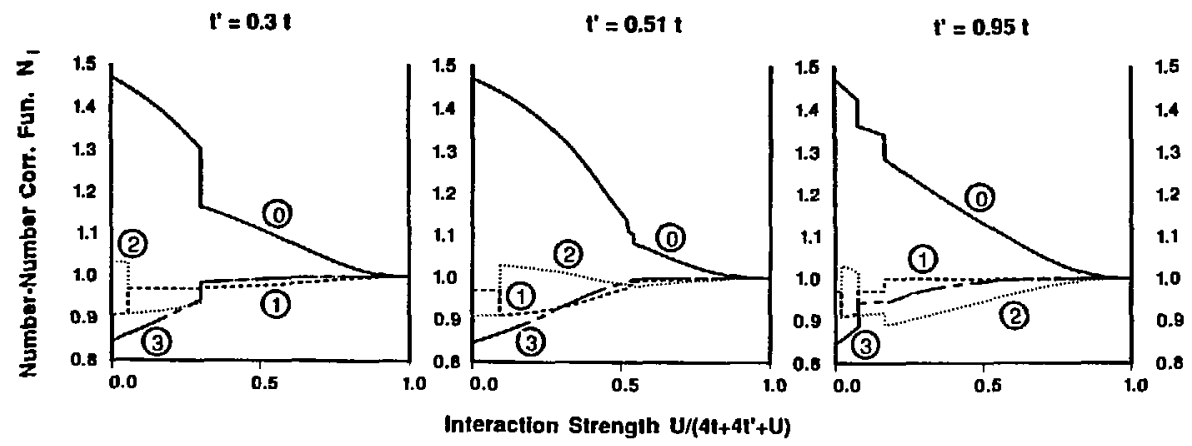


Figure 4.6. Ground-state phase diagram for the chiral Hubbard model at half-filling $(N=M=8)$. The vertical axis records the relative hopping [Eq. (4.19)] and the horjzontal axis records the interaction strength [Eq. (4.20)]. The labels denote the groundstate symmetry for each corresponding phase as given in Table 4.8. All ground states are spin singlets $(S=0)_{2}$ pseudospin singlets $(J=0)$, and have even particle-hole parity $(R=1)$. Adiabatic continuation is possible at three points: $t^{\prime}=0 ; t^{\prime}=t / 2$; and $t=0$.

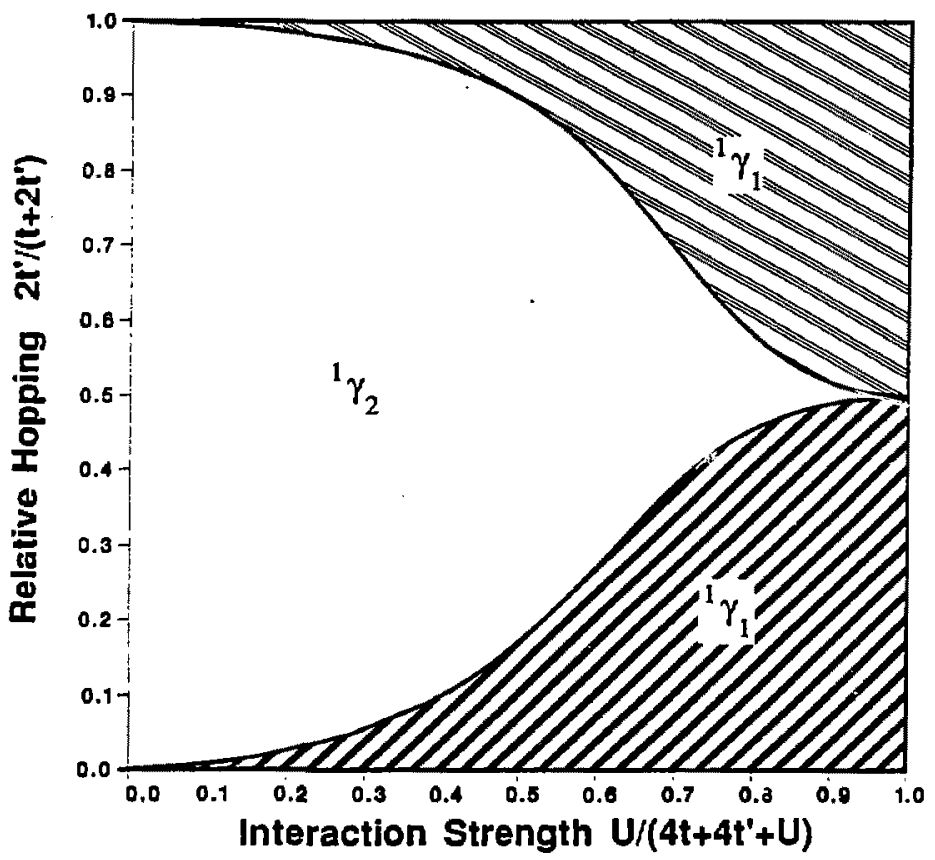


Figure 4.7. Spin-spin correlation functions $L_{i}$ [Eq. (4.16a)] for three different values of $t^{\prime} t t$ in the chiral Hubbard model. The point $r^{\prime}=0.3 t$ is representative of the case $t<t / 2$ and the point $t=0.8 t$ is representative of the case $t>t / 2$. The vertical axis plots the interaction strength [Eq. (4.20)] and the labels in the figures denote the subscript $i$. At large $U$ the ground state is ordered as a two-sublattice Néel antiferromagnet $(t<t / 2)$ or a four-sublattice Neel antiferromagnet $\left(l^{\prime}>t / 2\right)$. The point $r^{\prime}=t / 2$ is special and has intermediate-range antiferromagnetic order as $U \rightarrow \infty$. This may be a representative of a spin-liquid state for a finite system.

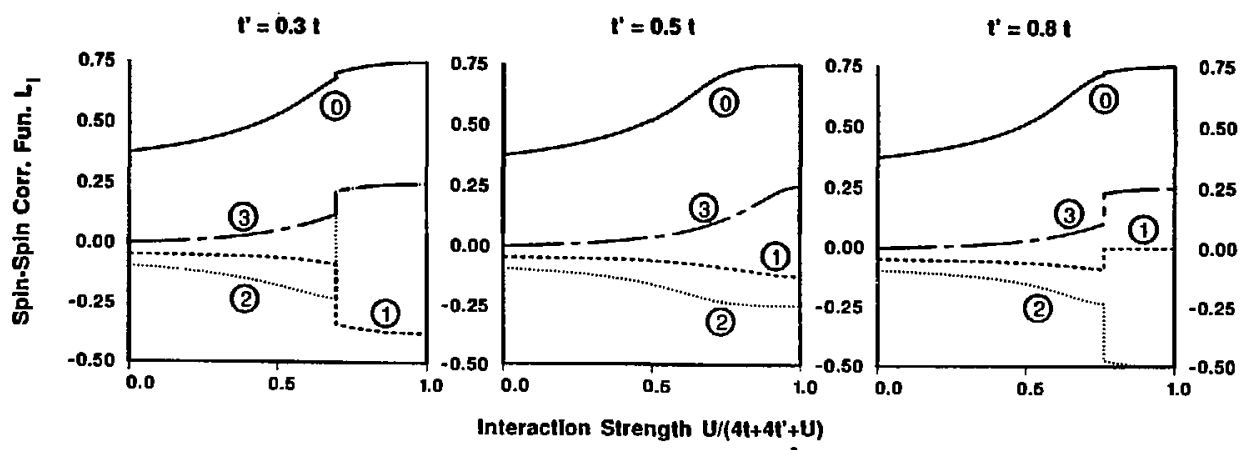


Figure 4.8. Number-number correlation functions $N_{i}$ [Eq. (4.16c)] for $t^{\prime}=0.3 t$ in the chiral Hubbard model. The other cases all have similar number-number correlation functions.

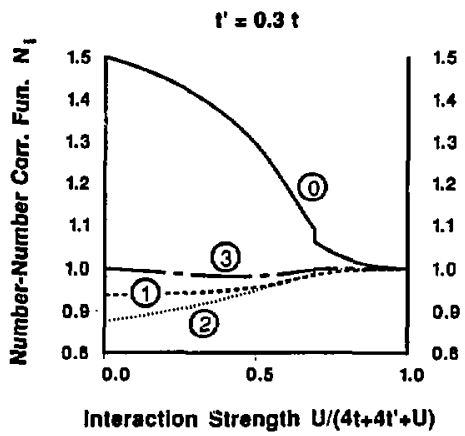


Figure 4.9. Spin-triple-product correlation function $O_{124}$ [Eq. (4.16d)] for two values of $t^{\prime} / t$ in the chiral Hubbard model. The point $t^{\prime}=0.3 t$ is representative of the general case where the sign of $O_{124}$ changes and the magnitude decreases by a factor of ten at the level crossing between the ${ }^{1} \gamma_{2}$ (small $U$ ) and the ${ }^{1} \gamma_{1}$ (large $U$ ) ground state. Note that at the special point $t=t / 2$ (where there is no level crossing) $O_{124}$ approaches zero with a fivite slope as $U \rightarrow \infty$.

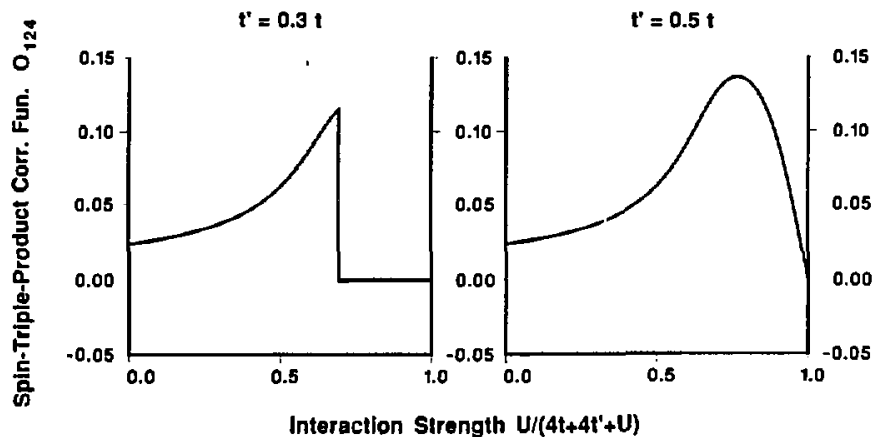




\section{Chapter V: Enlarged Symmetry Groups of Finite-Size Clusters with Periodic Boundary Conditions}

\section{V.1 Introduction}

The fundamental approximation for studying bulk properties of solid-state systems is the periodic crystal approximation. ${ }^{1}$ It has been used quite successfully in bandstructure calculations, ${ }^{2}$ Monte-Carlo simulations, ${ }^{3}$ and the small-cluster approach to the many-body problem. ${ }^{4}$ In the periodic crystal approximation an $M$-site crystal is modeled by a lattice of $M$ sites ${ }^{5}$ with periodic boundary conditions (PBC). Bloch's theorem ${ }^{6}$ then labels the quantum-mechanical wavefunctions by one of $M$ wavevectors in the Brillouin zone. In principle, a mactoscopic crystal is studied by taking the thermodynamic limit $(M \rightarrow \infty)$, which replaces the finite grid in reciprocal space by a continuum withir the Brillouin zone. In practice, the number of lattice sites is chosen to te as large as possible ( $M=$ finite), and the solution of the quantum-mechanical problem corresponds to a finite sampling in reciprocal space.

In the thermodynamic limit the complete symmetry group of the lattice is the space group, which is composed of all translations, rotations, and reflections that (rigidly) map the lattice onto itself and preserve its neighbor structure. In the case of a finite cluster, the complete symmetry group is a subgroup of $S_{M}$, the permutation group of $M$ elements, and is called the cluster-permutation group. The clusterpermutation group can be a proper subgroup of the syace group (i.e., it has fewer elements than the space group), contain operations that are not elements of the space group, or be identical to the space group. These three regimes are called, respectively, the self-contained-cluster regime, the high-symmetry regime, and the latrice regime. Note that the space group need not be a subgroup of the cluster-permutation group in the high-symmetry regime (although it usually is). 
A self-contained cluster is a cluster that does not add any new connections between lattice sites when PBC are imposed, but merely renormalizes parameters in the Hamiltonian. In this case, the cluster-pesmutation group is identical to the symmetry group of the same cluster with box boundary conditions. This symmetry group is, in turn, a point group (not necessarily the full point group of the lattice) with its origin at some point which can be called the center of the cluster, it is 2 proper subgroup of the space group. This phenomenon was first observed in the four-site square and tetrahedral clusters ${ }^{7}$ and in the eight-site simple-cubic cluster. ${ }^{8}$ The regime where the cluster-permutation group is a subgroup of the space group is called the selfcontained-cluster regime since every known example occurs in self-contained clusters.

The four-site square-lattice cluster is an example of a self-contained cluster. The lattice sites of the isolated cluster lie on the comers of a square and are numbered from one to four in a clockwise direction (ste Fig. 5.1a). When PBC are imposed (see Table 5.1) the four first-nearest-neighbors (1NN) of site-1 are two each of the sites 2 and 4 and the four second-nearest neighbors (2NN) are four each of site-3. Therefore, $1 \mathrm{NN}$ interactions must be renormalized by a factor of two and $2 \mathrm{NN}$ interacsions by a factor of four. Note that the imposition of PBC does not add any new connections to the lattice. The cluster-permutation group is isomorphic to the point group $C_{4 v}$ with an origin at the center of the square, the latter is a proper subgroup (order 8) of the space group (order 32).

The neighbor structure of a finite cluster is only defined out to the full extent of the cluster, i.e., the neighbor structure inciudes the minimal set of neighbor shells that exhaust all of the sites of the cluster. The neighbor structure for the four-site squarelattice cluster is recorded in Table 5.1. This information is, in fact, overcomplete since the entire lattice can be defined by the $1 \mathrm{NN}$ structure alone. Such a lattice is called a $1 \mathrm{NN}$-determined lattice and all known examples of self-contained clusters are 1NNdetermined lattices. 
There are two ways to generate symmetry operations that are not elements of the space group yielding the high-symmerry regime. The first possibility is that the lattice is not a $1 \mathrm{NN}$-determined lattice. In this case, there are always additional permutation operations that (nonrigidly) map the lattice onto itself and preserve the entire neighbor structure of the lattice. ${ }^{9}$ The size of the cluster-permutation group can be very large in this case. The second possibility occurs in a 1NN-determined lattice but the extra symmetry operations preserve only the 1NN structure of the lattice. ${ }^{10}$ A necessary (but not sufficient) condition for this phenomenon is given in the Appendix.

When the size of the cluster is large enough, the system enters the lattice regime with the cluster-permutation group identical to the space group. This always occurs because a large enough cluster is $1 N N$-determined and does not satisfy the necessary condition for extra symmetry operations given in the Appendix.

This contribution examines the implications of these extra symmetry operations to the quantum-mechanical solutions of Hamiltonians defined on small clusters. In the next section the transition from a self-contained system to an infinite lattice is studied for a class of cubic (and square) clusters and the consequences of the enlarged symmetry groups are outlined. Section V.3 follows this transition in detail for a squarelattice system. Section V.4 studies the eight-site clusters for the simple, body-centered, and face-centered cubic lattices and the square lattice. The group theory is applied to the many-body solutions of a model of strongly correlated electrons (the $t-t^{\prime}-J$ model). The final section contains a summary of the results and a conclusion.

\section{V.2 Group Theory for Cubic Clusters}

The transition from a self-contained cluster to a lattice is illustrated for the simplest set of simple $(s c)$, body-centered $(b c c)$, and face-centered $(f c c)$ cubic-lattice clusters and the square-lattice (sq) cluster. the set whose number of sites is a power of two $\left(M=2^{j}\right)$. Note that a subset of clusters (those with sizes that correspond to $j$ 
being a multiple of the spacial dimension of the lattice) are easily formed by repeatedly doubling the unit cell. For example, doubling the unit cell increases the cluster size by a factor of eight [four] for the cubic [square] systems.

The clusters for general $j$ are constructed as follows. A sc lattice is composed of two interpenetrating $f c c$ sublattices or four interpenetrating $b c c$ sublattices. Similarly, a bcc $[f c c]$ lattice is composed of two [four] interpenetrating se sublattices. A small cluster with $\mathrm{PBC}$ is constructed by decomposing an infinite lattice into $M$ interpenetrating sublattices (using the above decompositions) and assigning a different equivalence class (site number) to each of the $M$ sublanices. For example, an eightsite $f c c$-lattice cluster is constucted from four sc sublattices with each sc sublattice represented by two $f c c$ sublattices (see Fig. 3.3 of Chapter III). If each $s c$ sublattice is represented by four $b c c$ sublattices a sixteen-site $f c c$-lattice cluster is formed, and so on. The sq-lattice clusters (see Fig. 5.1) are constructed from $\sqrt{M} \times \sqrt{M}$ tilings of the plane that are aligned with [rotated by $45^{\circ}$ with respect to] the underlying square lattice for even [odd] $j$. In this fashion, every cluster whose number of sites is a power of two can be constructed except for the two-site $f c c$-lattice cluster.

Tables 5.2 and 5.3 summarize the results for the order of the cluster-permutation groups of the $s c, b c c, f c c$, and $s q$ lattice clusters as a function of cluster size. Table 5.2 corresponds to arbitrary Hamiltonians; Table 5.3 to Hamiltonians with $1 \mathrm{NN}$ interactions only. The self-contained-cluster regime corresponds to $M \leq 8[M \leq 4]$ for the $s c$ lattice [otherwise]. The high-symmetry regime is present at intermediate values of $M$ : for example, when the Hamiltonian contains only 1NN interactions, the highsymmery regime appears at $16 \leq M \leq 64$ for the sc lattice; $8 \leq M \leq 32$ for the bcc lattice; and $8 \leq M \leq 16$ for the $f c c$ and $s q$ lattices (see Table 5.3). The lattice regime. is entered for larger cluster sizes. The cluster-permutation group (in the highsymmery regime) has been studied previously for sq-Iattice clusters. ${ }^{11-13}$ 
A size range always exists where the lattice is not a 1NN-deternined lattice. In this case the order of the cluster-permutation group can be huge. For example, the sixteen-site $f c c$ lattice is composed of four interpenetrating $s c$ sublattices with each $s c$ lattice composed of four sites (four interpenetrating bcc sublattices). The 1NN of any site are the twelve sites that comprise the other three se sublattices. The secondnearest neighbors (2NN) are the three remaining sites of the original sc sublattice (each counted twice). Therefore, any permutation of the four elements within a sc sublartice or any permutation of the four $s c$ sublattices will commute with the Hamiltonian. The order of the cluster-permutation group is then $(4 !)^{5}=7,962,624$.

There are many implications that result from a cluster-permutation group that is not identical to the space group. In the self-contained-cluster regime, the clusterpermutation group is a subgroup of the space group, because some space-group operations are redundant (identical to the identity operation). Put in other words, a homomorphism exists between the space group and the cluster-permutation group with a nontrivial kemel composed of the redundant operations. This implies that only a subset of the irreducible representations of the space group (those that represent the sedundant operations by the unit matrix) are accessible to the solutions of the Hamiltonian. This process of rigorously eliminating irreducible representations as acceptable representations is well known. It occurs, for example, in systems that possess inversion s' nmetry: if the basis functions are inversion symmetric, then the system sustains only representations that are even under inversion.

In the high-symmetry regime, the cluster-permutation group $G$ contains operations that are not elements of the space group. The set $H$ of elements of the clusterpermutation group $G$ that are elements of the space group forms a subgroup of the cluster-permutation group that usually is equal to the space group. The group of translations forms an abelian invariant subgroup of $\boldsymbol{H}$ so that Bloch's theorem ${ }^{6}$ holds. The irreducible representations of $H$ are all irreducible representations of the space 
group. When the full cluster-permutation group $G$ is considered, the class structure of $H$ is expanded and modified, in general, with classes of $H$ combinilg together, and/or elements of $G$ outside of $H$ uniting with elements in a class of $H$, to form the new class structure of the cluster-permutation group $G$. The classes that contain the set of translations gpically contain elements that are not translations, so that the translation subgroup is no longer an invariant subgroup and representations of the clusterpermutation group cannot be constructed in the standard way. ${ }^{14}$ Furthermore, every irreducible representation of $\boldsymbol{H}$ that has nonuniform characters for the set of classes of $H$ that have combined to form one class of $G$ must combine with other irreducible representations to form a higher-dimensional irreducible representation of the clusterpermutation group. This phenomenon can be interpreted as a sticking together of irreducible representations of the space group arising from the extra (hidden) symmetry of the cluster.

There are further implications for short-ranged interactions. In the cases when the Hamiltonian has extra symmetry for INN-only interactions, the energy spectrum has levels that stick together in the absence of longer-ranged interactions and split as these interactions are turned on. However, the solutions will be nearly degenerate if the longer-ranged interactions are "weak" in relation to the $1 \mathrm{NN}$ interactions.

\section{V.3 Example: The Square Lattice}

The transition from the self-contained-cluster regime, through the high-symmetry regime, to the lattice regime is illustrated for the sq lattice. The four-site sq-lattice cluster (Fig. 5.1a) is a self-contained cluster. The space group is of order 32 and is composed of 14 classes. The Brillouin zone ${ }^{15}$ is sampled at three symmetry stars: $\Gamma$ $(\mathrm{d}=1) ; M(\mathrm{~d}=1)$; and $X(\mathrm{~d}=2)$. The origin of the space group is chosen to be site- 1 . One finds that the twofold rotation $\left\{C_{4}^{2} \mid 0\right\}$, and the reflections about the $x$ - and $y$ axes $\left\{\sigma_{x} \mid 0\right\},\left\{\sigma_{y} \mid 0\right\}$ are all redundant operations; i.e., they are identical to the 
identity operation $\{E \mid 0\}$, because the four-site cluster is self-contained. This implies that only irreducible representations of the space group that represent the twofold rotation and the reflections about the $x$ - and $y$-axes by the unit matrix are acceptable representations.

The character table of the full space group (with the acceptable representations highlighted in bold) is recorded in Table 5.4. The cluster-permutation group, with all repeated operations eliminated, is isomorphic to the point group $C_{4 \mathrm{v}}$ with its origin at the center of the square. Table 5.5 shows the mapping between the space-group notation and the point-group notation for the group elements. The acceptable space-group representations can now be identified with the more traditional point group representations: $\Gamma_{1} \rightarrow A_{1} ; \Gamma_{3} \rightarrow A_{2} ; M_{1} \rightarrow B_{1} ; M_{3} \rightarrow B_{2} ;$ and $X_{1} \rightarrow E$.

The eight-site $s q$-latrice cluster (Fig. 5.1b) is in the high-symmetry regime. The subgroup $H$ of the cluster-permutation group $G$ is the full space group, containing 64 elements distributed among 16 classes. The Brillouin zone ${ }^{15}$ is sampled at four symmetry stars: $\Gamma(\mathrm{d}=1) ; M(\mathrm{~d}=1) ; X(\mathrm{~d}=2)$; and $\Sigma(\mathrm{d}=4)$. The character table of $H$ may be found in Table 3.16 of Chapter III.

The eight-site sq-lattice cluster is not a $1 \mathrm{NN}$-determined lattice: if site-3 is placed arbitrarily on the lattice and its 1 NNs (sites 2, 4,6, and 8) are added, there are two inequivalent possibilities for the placement of the 2NN pair (sites 1 and 5). The permutation operator $P$ that interchanges site-1 with site-5 will map the lattice (nonrigidly) onto itself, preserving the entire neighbor structure of the lattice. The clusterpermutation group $G$ is then generated from the space group $H$ by closure. The existence of this nontrivial permutation operator is a finite-size effect of the eight-site cluster with PBC since it occurs because the lattice is not a 1NN-determined lattice.

The cluster-permutation group is composed of 128 elements divided into twenty classes and recorded in Table 5.6. Note that the presence of the permutation operator $\boldsymbol{P}$ forces physically different space-group operations (such as the translations, rotations 
and reflections) to be sometimes in the same class. In fact, four pairs of classes of $H$ combine to form single classes of $G$ (see Table 5.6): $(E \mid \tau)$ and $\left(\sigma \mid \tau_{\perp}\right\} ;\left\{C_{4} \mid \tau\right)$ and $\left\{\sigma^{\prime} \mid \tau\right\} ;\left\{C_{4}^{2} \mid \tau\right\}$ and $\left\{\sigma \mid \tau_{h}\right\}$; and $\{E \mid \theta\}$ and $\left\{C_{4}^{2} \mid \theta\right\}$. The translation subgroup is no longer an invariant subgroup and eight imeducible representations of $\boldsymbol{H}$ $\left(\Gamma_{2}, \Gamma_{4}, \Gamma_{5}, M_{2}, M_{4}, M_{5}, X_{3}\right.$, and $\left.X_{4}\right)$ must combine to form higher-dimens.unal representations of $G$. The character table is reproduced in Table 5.7 and includes the compatibility relations between representations of the cluster-permutation group $G$ and the space-group representations (of $H$ ) in the last column.

The case when the Hamiltonian contains only $1 \mathrm{NN}$ interactions has an enlarged symmetry group since the eight-site sq-lattice cluster with 1NN-only interactions is identical to a $b c c$-lattice cluster with $1 \mathrm{NN}$-only interactions ${ }^{8}$ (see Section V.4).

The sixteen-site sq-lattice cluster (Fig. 5.1c) is in the lattice regime for arbitrary interactions. There is no extra symmetry beyond the space-group symmetry and furcher analysis proceeds in a standard fashion [the Brillouin zone ${ }^{15}$ is sampled at six symmetry stars: $\Gamma(\mathrm{d}=1) ; M(\mathrm{~d}=1) ; X(\mathrm{~d}=2) ; \Sigma(\mathrm{d}=4) ; \Delta(\mathrm{d}=4) ;$ and $Z(\mathrm{~d}=4)$ ]. Hidden symmetry exists when the Hamiltonian is restricted to $1 \mathrm{NN}$-interactions only. The cluster-permutation group then contains 384 elements divided into twenty classes. The nonsigid permutation operator that generates the cluster-permutation group from the space group is given in the Appendix. This group is identical to the point group of a four-dimensional hypercube ${ }^{12}$ but will not be pursued furcher here.

\section{V.4 Example: Eight-Site Clusters and the $t-t^{\prime}-J$ Model}

As a further illustration, the eight-site clusters are examined in more detail. The se-lattice cluster is a self-contained cluster (see Fig. 3.1 of Chapter III). The point group operations (with origin at a lattice site) corresponding to a rotation by $180^{\circ}$ about the $x-, y$-, or $z$-axis $\left(C_{4}^{2} \mid 0\right\}$ and the inversion $(J \mid 0)$ are all redundant operations; i.e., they are identical to the identity operation $\{E \mid 0\}$. Therefore, only 
ireducible representations of the space group that represent $\left\{C_{4}^{2} \mid 0\right\}$ and $[J \mid 0\}$ by the unit matrix are acceptable representations. This is summarized in the character table for the cluster-permutation group (see Table 3.13 in Chapter III). The clusterpermutation group is isomorphic to the full cubic point group, $O_{h}$, with an origin at the center of the cube defined by the eight sides of the cluster.

The $b c c-, f c c$-, and $s q$-lattice clusters are all in the high-symmerry regime. The $b c c$-latice is constructed from two four-site sublattices. The points in the $b c c$ Brillouin zone $e^{15}$ sampled here are $\Gamma, H$, and $N$. The cluster-permutation group includes any independent permutation of the elements within each sublattice and the interchange of the two sublattices. The subgroup $H$ corresponds to all translations and proper rotations and contains the following fourteen representations (with corresponding dimensions in parentheses): $\Gamma_{1}(1) ; \Gamma_{2}(1) ; \Gamma_{12}(2) ; \Gamma_{1 s^{\prime}}(3) ; \Gamma_{2 s^{\prime}}(3) ; H_{1}(1) ; H_{2}$ (1); $H_{12}(2) ; H_{15}^{\prime}(3) ; H_{25}{ }^{\prime}(3) ; N_{1}(6) ; N_{2}(6) ; N_{3}(6) ;$ and $N_{4}(6)$. The character table is given in Table 3.14 of Chapter III. The classes $[E \mid \tau)$ and $\left\{C_{2} \mid \tau_{1}\right\}$ combine to form one class of the cluster-permutation group as do the two classes $\{E \mid \theta\}$ and $\left\{C_{4}^{2} \mid \theta_{1}\right\}$ and the three classes $\left\{C_{4} \mid \tau\right\},\left\{C_{4}^{2} \mid \tau\right\}$, and $\left\{C_{2} \mid \tau_{L}\right\}$. The only representations that are not required to stick together are then $\Gamma_{1}, H_{1}, N_{1}$, and $N_{4}$. The cluster-permutation group has twenty irreducible representations that satisfy the compatibility relations with $H$ given in Table 5.8.

The $f c c$-lattice cluster-permutation group ${ }^{16}$ has a subgroup if that corresponds to all translations and proper rotations (see Table 3.15 of Chapter III) and is generated by the space-group generators and the permutation operator $P$ that transposes the originwith its $2 \mathrm{NN}$. There are twenty irreducible representations as recorded in Table 5.8.

The sq-latrice cluster-permutation group has been srudied in detail in Section V.3. The compatibility relacions of the twenty irreducible representations can be found in the last column of the character table (Table 5.7). Note that in the case of INN-only interactions the eight-site $s q$-lattice cluster is identical to the eight-site bcc-lattice 
cluster. $^{8}$

As an application of these enlarged symmetry groups, a model of strong electron correlation (the $t-t^{\prime}-J$ model) is studied on these eight-site clusters. The $t--t^{\prime}-J$ model involves hopping between INN and between 2NN (excluding any doubleoccupation of a site) and a Heisenberg antiferromagnetic INN exchange interaction. Previous work on this model ${ }^{8}$ utilized only the symmetry of the subgroup $H$ of the space group. Use of the cluster-permutation group simplifies the problen even further and explains most of the "accidental" degeneracies observed in the many-body energy levels. The largest Hamiltonian blocks that need to be diagonalized after the clusterpermutation group symmetry is incorporated are as follows: $5 \times 5$ for five electrons in the bcc-lattice; $7 \times 7$ for six electrons in the $f c c$-lattice; and $11 \times 11$ for six electrons in the $s q$-lattice. It is interesting to note that, with the exception of two $5 \times 5$ blocks, the 6,561 $\times 6,561$ Hamiltonian matrix can be diagonalized analytically for the bcclattice.

There are only a few cases of extra degeneracies that remain in the energy spectrum. Most of these degeneracies involve parameter-independent eigenvectors; i.e., eigenvectors that do not depend on the hopping integrals $t$ or $t$ or on the Heisenberg antiferromagnetic interaction $J$. The $f c c$ - and $s q$-lattices both have parameterdependent eigenstates with energy levels that stick together and are summarized in Table 5.9. This sticking-together ${ }^{17}$ of levels would be explained if there was a larger symmetry group, an orbital-permutation group, that involves permutations mixing spatial and spin degrees of freedom, and contains the cluster-permutation group as a subgroup. The evidence in favor of this conjecture is that the extra degeneracies oceur only between specific cluster-permutation group representations that have the sume total spin. ${ }^{18}$ A similar phenomenon was observed in the Hubbard model at half-filling on an eight-site sq-lattice cluster. ${ }^{13}$ 


\section{V.5 Conclusions}

This contribution outlines the transition from a system that resembles an isolated cluster (point-group symmetry) to a system that resembles an infinite lattice (spacegroup symmetry). An intermediate region is discovered that has increased symmetry beyond that of the space group. These additional symmetry operations are nonrigid . transformations that map the cluster onto itself and form a group, the clusterpermutadion group (which typically includes the space group as a subgroup). An analysis of the cluster-permutation group shows two different effects: (1) the Eamiltonian matrix for a given representation of the space group may split into irreducible blocks; and (2) irreducible representations of the space group (which frequently correspond to different points in the Brillouin zone) may "stick together." These two effects explain several puzzling degeneracies and level-crossings found, numerically or analytically, in many cluster calculations.

The order of the cluster-permitation group may be quite large (see for example, the group of order $7,962,624$ for the sixteen-site cluster in the $f c c$-latticis). The extra symmetry of such a large group greatly facilitates the numerical problem of diagonalizing large Hamiltonians and may result in completely analytical solutions (as seen in the eight-site cluster in the $f c c$-lattice ${ }^{18}$ ). The size of the cluster may be fairly large before this extra symmetry is lost (it survives up to the sixty-four-site sc-lattice cluster for Hamiltonians with $1 \mathrm{NN}$ interactions only). The effect of an enlarged symmetry group is more pronounced in systems with short-range-only interactions (compare Tables 5.2 and 5.3) since many nonrigid transformations that map the cluster onto itself preserve only the $1 \mathrm{NN}-\mathrm{structure}$ of the lattice.

The transition from the self-contained-cluster regime, through the hiddensymmetry regime, to the lattice regime were studied explicitly for the two-dimensional square lattice. The group theory for the eight-site clusters in the simple, bodycentered, and face-centered cubic lattices and in the square lattice were discussed in 
detail and applied to a model of strong electron correlation (the $t-t^{\prime}-J$ model). Mcst "accidental" degeneracies of the many-body energy levels are now explained. There is a strong indication that additional hidden symmetry remains in the $f c c$ - and sq-lattices that mixes spatial and spin degrees of freedom.

\section{V.6 Appendix: Linear-Pair Rule}

A necessary (but not sufficient) condition for extra symmetry operations is the linear-pair rule: A linear pair is defined to be a pair of distinct opposite INN of a lattice site (i.e., the linear pair and the chosen lattice site all lie on a line). An infinite lattice has one unique lattice site that has both elements of the linear pair as 1NN. The linear-pair I is satisfied whenever there is more than one lattice site that has both elements of ... linear pair as 1 NN. If the linea r ule is satisfied, then the cluster-permutation group may contain elements outside $c$. the space group for Hamiltonians that inciude only $1 \mathrm{NN}$ interactions, but is a (proper or improper) subgroup of the space group otherwise.

The nonrigid permutation operations that can be constructed when the lattice satisfies the linear-pair rule involve a nonrigid transformation of the INN of a give.. site. If a permutation operation can be constructed that interchanges $1 \mathrm{NN}$ of a given site so that elements that initially formed a linear pair do not form a linear pair after the permutation, and this operation can be completed (consistently) to the entire cluster (preserving the 1NN-structure of the lattice), then a nonrigid permutation operator has been discovered.

As an example, consider the sixteen-site sq-lattice cluster (Fig. 5.1c). The lineas-pair sule is satisfied since both elements of the linear pair $(2,4)$ are $1 \mathrm{NN}$ to the sites 1 and 3. The permutation operator

$$
\left[\begin{array}{cccccccccccccccc}
1 & 2 & 3 & 4 & 5 & 6 & 7 & 8 & 9 & 10 & 11 & 12 & 13 & 14 & 15 & 16 \\
11 & 12 & 13 & 14 & 3 & 4 & 5 & 6 & 7 & 8 & 1 & 2 & 15 & 16 & 9 & 10
\end{array}\right]
$$


is an order-six element that corresponds to a nonrigid transformation of the sq-lattice cluster onto itself preserving the 1NN-structure of the lattice. It will generate the entire cluster-permutation group from the space group by closure.

The linear-pair rule is not a sufficient condition to produce extra symmetry for 1NN-only interactions since the sixty-four-site bcc-lattice and the thirty-two- and sixty-four-site $f c c$-lattice clusters all satisfy th: linear-pair rule, but do not have any additional symmetry beyond the space group (see Tables 5.2 and 5.3).

It is interesting to note that the $f c c$ latrice is the only lattice that has no extra symmetry for 1NN-only interactions (compare Tables 5.2 and 5.3). This probably arises because the $f c c$ lattice is not bipartite. ${ }^{19}$ 


\section{V.7 References for Chapter V}

1 L.M. Falicov, Group Theory and Its Physical Applications, (University of Chicago Press, Chicago, 196i) p. 144ff.

2 J. Callaway, Quantum Theory of the Solid State, (Academic Press, San Diego, 1974) Ch. 4.

3 Monte Carlo Methods in Quantum Problems, edited by M.H. Kalos, NATO ASI Series C, Vol. 125 (Reidel, Dordrecht, 1984).

4 For a review see L.M. Falicov in Recent Progress in Many-Body Theories Vol. 1, sdited by A.J. Kallio, E. Pajanne, and R.F. Bishop, (Plenum, New York, 1988) p. 275; J. Callaway, Physica B 149, 17 (1988).

5 Lattices are chosen that are compatible with the space group of the infinite lattice; i.e., the lattice is mapped onto itself by every element of the space group (not necessarily in a unique fashion). For example, a rectangular cluster with periodic boundary conditions would not be allowed as an approximation to a square lattice since a $90^{\circ}$ rotation does not map the cluster onto itself.

6 C. Kittel, Quantum Theory of Solids, (Wiley, New York, 1987) pp. $179 f f$.

7 A.M. Oles, B. Oles, and K.A. Chao, J. Phys. C 13, L979 (1980); J. Rbssler, B. Fernandez, and M. Kini, Phys. Rev. B 24, 5299 (1981); D.J. Newman, K.S. Chan, and B. Ng, J. Phys. Chem. Solids 45, 643 (1984); L.M. Falicov and R.H. Victora, Phys. Rev. B 30, 1695 (1984).

B J.K. Freericks and L.M. Falicov, Phys. Rev. B 42, 4960 (1990).

9 There may be additional nonrigid operations that preserve only the INN structure of the lattice.

10 An exception to the second possibility is the eight-site $f(c$-lattice cluster. It is a 1NN-determined lerrice, but the cluster-permutation group preserves the entire neighbor-structure of the lattice. The reason is each site has six sites that are 
1NNs and one site that is a $2 \mathrm{NN}$. Therefore, any permutation operation that preserves the 1NN-structure must, by default, also preserve the $2 \mathrm{NN}$-structure.

11 Although the ten-site sq-lattice eluster does not satisfy the criterion of Ref. 5, the cluster-permutation group does sustain operations that are not elements of the space group, as discussed by R. Saito, Sol. St. Commun. 72, 517 (1989); T. Ishino, R. Saito, and H. Kamimura, J. Phys. Soc. Japan, 59, 3886 (1990).

12 The presence of additional symmetry for a sixteen-site $s q$-lattice cluster with 1NN-only interactions is noted by J.A. Riera and A.P. Young, Phys. Rev. B 39, 9697 (1989).

13 The complete group theory for the eight-site sq-lattice cluster has been examined by J.K. Freericks, L.M. Falicov, and D.S. Rokhsar, unpublished.

14 L.M. Falicov, Group Theory and Its Physical Applications, (University of Chicago Press, Chicago, 1966) p. 151ff.

15 L.P. Bouckaert, R. Smoluchowski, and E.P. Wigner, Phys. Rev. 50, 58 (1936).

16 J.K. Freericks and L.M. Falicov, unpublished.

17 Energy levels of a different symmetry can cross, but cannot be degenerate for all values of the parameters, as discussed by O.J. Heilmann and E.H. Lieb, Trans. N.Y. Acrd. Sci. 33, 116 (1971).

The many-body energy levels of the seven-electron case of the $t-t^{t}-J$ model on the $f c c$-lattice have been analysically determined by A. Reich and L.M. Falicov, Phys. Rer. B 35, 5560 (1988); ibid., 38, 11199 (1988). The largest Hamiltonian block is $24 \times 4$ block when the full cluster-permutation-group symmetry is taken into account. There appears to be even more hidden symmetry, however, as the largest secular equation is a quadraric equation. Since the cluster-permutation group exhausts all of the spatial symmetry, any additional symmetry must mix spatial and spin degrees of freedom. 
19 E.H. Lieḅ, Phys. Rev. Lett. 62, 1201 (1989). 
V.7 Tables for Chapter V

Table 5.1. Neighbor structure for the four-site square-lattice cluster.

\begin{tabular}{c|rrrr|rrrr}
\hline \hline site & \multicolumn{4}{c}{1 1NN } & \multicolumn{4}{c}{ 2NN } \\
\cline { 2 - 8 } 1 & 2 & 2 & 4 & 4 & 3 & 3 & 3 & 3 \\
2 & 1 & 1 & 3 & 3 & 4 & 4 & 4 & 4 \\
3 & 2 & 2 & 4 & 4 & 1 & 1 & 1 & 1 \\
4 & 1 & 1 & 3 & 3 & 2 & 2 & 2 & 2 \\
\hline
\end{tabular}


Table 5.2. Order of the cluster-permutation group for arbitrary interactions on finitesize clusters with periodic boundary conditions of the simple, body-centered, and facecentered cubic lattices and of the two-dimensional square lattice. The symbols $S, I i$, and $L$ denote the self-contained, high-symmetry, and lattice regirnes, respectively. The cases with cluster sizes larger than 32 are in the lattice regime.

\begin{tabular}{c|c|cc|cc|ccc|cc|c}
\hline $\begin{array}{c}\text { cluster } \\
\text { size }\end{array}$ & $\begin{array}{c}\text { cubic } \\
\text { space group }\end{array}$ & \multicolumn{2}{c}{$s c$} & \multicolumn{2}{c}{ bcc } & \multicolumn{2}{c}{$f c c$} & \multicolumn{2}{c}{$\begin{array}{c}\text { square } \\
\text { space group }\end{array}$} & $s q$ \\
\hline 1 & 48 & $\mathrm{~S}$ & 1 & $\mathrm{~S}$ & 1 & $\mathrm{~S}$ & 1 & 8 & $\mathrm{~S}$ & 1 \\
2 & 96 & $\mathrm{~S}$ & 2 & $\mathrm{~S}$ & 2 & - & - & 16 & $\mathrm{~S}$ & 2 \\
4 & 192 & $\mathrm{~S}$ & 24 & $\mathrm{~S}$ & 8 & $\mathrm{~S}$ & 24 & 32 & $\mathrm{~S}$ & 8 \\
8 & 384 & $\mathrm{~S}$ & 48 & $\mathrm{H}$ & 1,152 & $\mathrm{H}$ & 384 & 64 & $\mathrm{H}$ & 128 \\
16 & 768 & $\mathrm{H}$ & 12,288 & $\mathrm{H}$ & 4,608 & $\mathrm{H}$ & $7,962,624$ & 128 & $\mathrm{~L}$ & 128 \\
32 & 1,536 & $\mathrm{~L}$ & 1,536 & $\mathrm{~L}$ & 1,536 & $\mathrm{~L}$ & 1,536 & 256 & $\mathrm{~L}$ & 256 \\
\hline \hline
\end{tabular}


Table 5.3. Order of the cluster-permutation group for 1NN-only interactions on finitesize clusters with periodic boundary conditions of the simple, body-centered, and facecentered cubic lattices and of the two-dimensional square lattice. The symbols $S, H$, and $L$ denote the self-contained, high-symmetry, and lattice regimes, respectively. The cases with cluster sizes larger than 128 are in the lattice regime.

\begin{tabular}{c|c|cc|cc|cc|ccc}
\hline $\begin{array}{c}\text { cluster } \\
\text { size }\end{array}$ & $\begin{array}{c}\text { cubic } \\
\text { space group }\end{array}$ & \multicolumn{2}{c}{$s c$} & \multicolumn{1}{c}{ bcc } & \multicolumn{4}{c}{$\begin{array}{c}\text { square } \\
\text { space group }\end{array}$} & $s q$ \\
\hline 1 & 48 & S & 1 & S & 1 & S & 1 & 8 & S & I \\
2 & 96 & S & 2 & S & 2 & - & - & 16 & S & 2 \\
4 & 192 & S & 24 & S & 8 & S & 24 & 32 & S & 8 \\
8 & 384 & S & 48 & H & 1,152 & H & 384 & 64 & H & 1,152 \\
16 & 768 & H & 12,288 & H & $3,251,404,800$ & H & $7,962,624$ & 128 & H & 384 \\
32 & 1,536 & H & 13,824 & H & 6,144 & L & 1,536 & 256 & L & 256 \\
64 & 3,072 & H & 27,648 & L & 3,072 & L & 3,072 & 512 & L & 512 \\
128 & 6,144 & L & 6,144 & L & 6,144 & L & 6,144 & 1,024 & L & 1,024 \\
\hline
\end{tabular}


Table 5.4. Character table for the space group of the four-site cluster on the square latice. The symbol $\sigma$ denotes the mirror planes perpendicular to the $x$ - and $y$-axes and $\sigma$ denotes the mirror planes perpendicular to the diagonals $x \pm y$. The translations are denoted by 0 (no translation), $\tau$ (first-nearest-neighbor translation), and $\theta$ (secondnearest-neighbor). The subscripts If and 1 refer to translations parallel to or perpendicular to the normais of the mirror planes. The acceptable representations of the space group, that form the representations of the cluster-permutation group, are highlighted in bold.

\begin{tabular}{lrrrrrrrrrrrrrrr}
\hline \hline \multicolumn{1}{l}{} & 1 & 1 & 2 & 1 & 1 & 2 & 4 & 4 & 2 & 2 & 2 & 2 & 4 & 4 \\
& $E$ & $C_{4}^{2}$ & $\sigma$ & $E$ & $C_{4}^{2}$ & $\sigma$ & $C_{4}$ & $\sigma$ & $E$ & $C_{4}^{2}$ & $\sigma$ & $\sigma$ & $C_{4}$ & $\sigma^{\prime}$ \\
& 0 & 0 & 0 & $\theta$ & $\theta$ & $\theta$ & $\tau$ & $\tau$ & $\tau$ & $\tau$ & $\tau_{1}$ & $\tau$ & $0 \theta$ & $0 \theta$ \\
\hline$\Gamma_{1}$ & 1 & 1 & 1 & 1 & 1 & 1 & 1 & 1 & 1 & 1 & 1 & 1 & 1 & 1 \\
$\Gamma_{2}$ & 1 & 1 & -1 & 1 & 1 & -1 & 1 & -1 & 1 & 1 & -1 & -1 & 1 & -1 \\
$\Gamma_{3}$ & 1 & 1 & 1 & 1 & 1 & 1 & -1 & -1 & 1 & 1 & 1 & 1 & -1 & -1 \\
$\Gamma_{4}$ & 1 & 1 & -1 & 1 & 1 & -1 & -1 & 1 & 1 & 1 & -1 & -1 & -1 & 1 \\
$\Gamma_{5}$ & 2 & -2 & 0 & 2 & -2 & 0 & 0 & 0 & 2 & -2 & 0 & 0 & 0 & 0 \\
& & & & & & & & & & & & & & \\
$\mathbf{M}_{1}$ & 1 & 1 & 1 & 1 & 1 & 1 & -1 & -1 & -1 & -1 & -1 & -1 & 1 & 1 \\
$M_{2}$ & 1 & 1 & -1 & 1 & 1 & -1 & -1 & 1 & -1 & -1 & 1 & 1 & 1 & -1 \\
$\mathbf{M}_{3}$ & 1 & 1 & 1 & 1 & 1 & 1 & 1 & 1 & -1 & -1 & -1 & -1 & -1 & -1 \\
$M_{4}$ & 1 & 1 & -1 & 1 & 1 & -1 & 1 & -1 & -1 & -1 & 1 & 1 & -1 & 1 \\
$M_{5}$ & 2 & -2 & 0 & 2 & -2 & 0 & 0 & 0 & -2 & 2 & 0 & 0 & 0 & 0 \\
& & & & & & & & & & & & & & \\
$\mathbf{X}_{1}$ & 2 & 2 & 2 & -2 & -2 & -2 & 0 & 0 & 0 & 0 & 0 & 0 & 0 & 0 \\
$X_{2}$ & 2 & 2 & -2 & -2 & -2 & 2 & 0 & 0 & 0 & 0 & 0 & 0 & 0 & 0 \\
$X_{3}$ & 2 & -2 & 0 & -2 & 2 & 0 & 0 & 0 & 0 & 0 & 0 & 0 & 0 & 0 \\
$X_{4}$ & 2 & -2 & 0 & -2 & 2 & 0 & 0 & 0 & 0 & 0 & 0 & 0 & 0 & 0 \\
\hline \hline
\end{tabular}


Table 5.5. Repeated operations of the space group for the four-site cluster in the square latice and their identification with point-group operations. The clusterpermutation group is isomorphic to the point group $C_{4 v}$ with an origin at the center of the square. The space-group operations are denoted in the standarl notation of a point-group operation followed by a translation all enclosed in braces. Put in more mathematical terms, this table explicitly lists the homomorphism that maps the space group onto the cluster-permutation group. The first row (corresponding to the redundant operations of the space group) forms the kernel of the homomorphism.

\begin{tabular}{c|ccc}
\hline $\begin{array}{c}\text { point-group } \\
\text { operation }\end{array}$ & \multicolumn{3}{c}{$\begin{array}{c}\text { space-group } \\
\text { operations }\end{array}$} \\
\hline$E$ & $\{E \mid 0\}$, & $\left\{C_{4}^{2} \mid 0\right\}$, & $\{\sigma \mid 0\}$ \\
$C_{4}^{2}$ & $\{E \mid \theta\}$, & $\left\{C_{4}^{2} \mid \theta\right\}$, & $\{\sigma \mid \theta\}$ \\
$C_{4}$ & $\left\{C_{4} \mid \tau\right\}$, & $\left\{\sigma^{\prime} \mid \tau\right\}$ & \\
$\sigma$ & $\{E \mid \tau\}$, & $\left\{C_{4}^{2} \mid \tau\right\}$, & $\{\sigma \mid \tau\}$ \\
$\sigma^{\prime}$ & $\left\{C_{4} \mid 0, \theta\right\}$, & $\left\{\sigma^{\prime} \mid 0, \theta\right\}$ & \\
\hline
\end{tabular}


Table 5.6. Class structure and group elements of the 128 element cluster-permutation group of the eight-site square-lattice ciuster. The notation is the same as that of Table 5.4 and $\Omega$ denotes the third-nearest-neighbor translation. The element $P$ corresponds to the transposition of site-1 and site-5 (see Fig. 5.16).

\begin{tabular}{|c|c|c|c|c|}
\hline class & & group elements & & size of class \\
\hline 1 & $\{E \mid 0\}$ & & & 1 \\
\hline 2 & $\left\{C_{4} \mid 0, \theta, \Omega\right\}$ & & & 8 \\
\hline 3 & {$\left[C_{4}^{2} \mid 0, \Omega\right]$} & & & 2 \\
\hline 4 & $\{\sigma \mid 0, \Omega\}$ & & & 4 \\
\hline 5 & $\left\{\sigma^{\prime} \mid 0, \theta_{\perp}\right\}$ & & & 4 \\
\hline 6 & $\{E \mid \tau\}$ & {$\left[\sigma \mid \tau_{1}\right]$} & & 8 \\
\hline 7 & $\left\{C_{4} \mid \tau\right)$ & $\{\sigma \mid \tau)$ & & 16 \\
\hline 8 & $\left\{C_{4}^{2} \mid \tau\right\}$ & $\left\{\sigma \mid \tau_{k}\right\}$ & & 8 \\
\hline 9 & $\{E \mid \theta\}$ & $\left\{C_{4}^{2} \mid \theta\right\}$ & & 4 \\
\hline 10 & $\{\sigma \mid \theta\}$ & & & 4 \\
\hline 11 & $\left\{\sigma^{\prime} \mid \theta_{\|}, \Omega\right\}$ & & & 4 \\
\hline 12 & $\{E \mid \Omega\}$ & & & 1 \\
\hline 13 & $P\{E \mid 0\}$ & $P\left\{C_{4}^{2} \mid \Omega\right\}$ & $P[\sigma \mid \Omega\}$ & 4 \\
\hline 14 & $P\left\{C_{4} \mid 0\right\}$ & $P\left(\sigma^{\prime} \mid \theta_{\|}\right\}$ & & 4 \\
\hline 15 & $P\left(C_{4}^{2} \mid 0\right\}$ & $P\{\sigma \mid 0\}$ & $P\{E \mid \Omega\}$ & 4 \\
\hline 16 & $P\left\{\sigma^{\prime} \mid 0, \Omega\right]$ & $P\left\{C_{4} \mid \theta\right\}$ & & 8 \\
\hline 17 & $P\{E \mid \tau\}$ & $P\left\{C_{4}^{2} \mid \tau\right\}$ & $P(\sigma \mid \tau)$ & 16 \\
\hline 18 & $P\left(C_{4} \mid \tau\right\}$ & $P\left(\sigma^{\prime} \mid \tau\right]$ & & 16 \\
\hline 19 & $P[E \mid \theta]$ & $P\left\{C_{4}^{2} \mid \theta\right\}$ & $P(\sigma \mid \theta)$ & 8 \\
\hline 20 & $P\left(C_{4} \mid \Omega\right)$ & $P\left[\sigma^{\prime} \mid \theta_{\downarrow}\right]$ & & 4 \\
\hline
\end{tabular}


Table 5.7. Character table of the 128 element clustror-permutation group for the eightsite square-lattice cluster. The class structure and group elements are given in Table 5.6. The classes are labeled by their number to save space in the table below. The last column gives the compatibility relations with the irreducible representations of the space group $H$ (Table 3.16 of Chapter III). The subscripts $p, z$, and $n$ denote representations that have a positive character, zero character, or negative characier, respectively, for the element $P[E \mid 0]$. The symbol $\phi$ is used to denote representations that mix different wavevectors. 


\begin{tabular}{|c|c|c|c|c|c|c|c|c|c|c|c|c|c|c|c|c|c|c|c|c|c|}
\hline & $\begin{array}{c}1 \\
c_{1}\end{array}$ & $\begin{array}{r}8 \\
c_{2} \\
\end{array}$ & $\begin{array}{r}2 \\
c_{3} \\
\end{array}$ & $\begin{array}{r}4 \\
\angle 4 \\
\end{array}$ & $\begin{array}{r}4 \\
c_{5}\end{array}$ & $\begin{array}{r}8 \\
c_{6} \\
\end{array}$ & $\begin{array}{l}16 \\
c ?\end{array}$ & $\begin{array}{r}8 \\
6\end{array}$ & $\begin{array}{r}4 \\
c_{9} \\
\end{array}$ & $\begin{array}{c}4 \\
c^{10}\end{array}$ & $\begin{array}{c}4 \\
c_{1} !\end{array}$ & $\begin{array}{c}1 \\
c_{12}\end{array}$ & $\begin{array}{r}4 \\
c_{13} \\
\end{array}$ & $\begin{array}{r}4 \\
c_{14} \\
\end{array}$ & $\begin{array}{c}4 \\
c_{13}\end{array}$ & $\begin{array}{c}8 \\
c_{16} \\
\end{array}$ & $\begin{array}{l}16 \\
c_{17}\end{array}$ & $\begin{array}{l}16 \\
c_{11}\end{array}$ & $\begin{array}{c}8 \\
c_{19} \\
\end{array}$ & $\begin{array}{r}4 \\
c_{20} \\
\end{array}$ & \\
\hline$r_{1, p}$ & 1 & 1 & 1 & 1 & 1 & 1 & 1 & 1 & 1 & 1 & 1 & 1 & 1 & 1 & 1 & 1 & 1 & 1 & 1 & 1 & $\Gamma_{1}$ \\
\hline$r_{i n}$ & 1 & 1 & 1 & 1 & 1 & 1 & 1 & 1 & 1 & 1 & 1 & 1 & 1 & +1 & -1 & -1 & -1 & -1 & -1 & -1 & $r_{1}$ \\
\hline$\Gamma_{3}$ & 1 & -1 & 1 & 1 & -1 & 1 & -1 & 1 & 1 & I & -1 & 1 & i & -1 & 1 & -1 & 1 & -1 & 1 & -1 & $\Gamma_{3}$ \\
\hline$\Gamma_{3 n}$ & 1 & -1 & 1 & 1 & -1 & 1 & -1 & 1 & 1 & 1 & -1 & 1 & -1 & 1 & -1 & 1 & -1 & I & -1 & 1 & $\Gamma_{3}$ \\
\hline$M_{1,}$ & I & 1 & 1 & 1 & 1 & -1 & -1 & -1 & 1 & 1 & 1 & 1 & 1 & 1 & 1 & 1 & +1 & -1 & 1 & 1 & $\overrightarrow{n i}_{1}$ \\
\hline$M_{1 n}$ & I & 1 & 1 & 1 & 1 & -1 & -1 & -1 & I & 1 & $\mathbf{I}$ & 1 & -1 & -1 & -1 & -1 & I & 1 & -1 & -1 & $\mu_{1}$ \\
\hline$M_{3 p}$ & 1 & -1 & 1 & 1 & -1 & -1 & 1 & -1 & 1 & 1 & -1 & 1 & 1 & -1 & 1 & -1 & .1 & 1 & 1 & -1 & $M_{3}$ \\
\hline$M_{3 n}$ & 1 & -1 & 1 & 1 & -1 & -1 & 1 & -1 & 1 & 1 & -1 & 1 & -1 & 1 & -1 & 1 & 1 & -1 & -1 & 1 & $M_{3}$ \\
\hline$\phi_{1}$ & 2 & 2 & 2 & -2 & -2 & 0 & 0 & 0 & 2 & -2 & -2 & 2 & 0 & 0 & 0 & 0 & 0 & 0 & 0 & 0 & $\Gamma_{2} \oplus M_{2}$ \\
\hline$\phi_{2}$ & 2 & -2 & 2 & -2 & 2 & 0 & 0 & 0 & 2 & -2 & 2 & 2 & 0 & 0 & 0 & 0 & 0 & 0 & 0 & $\mathbf{0}$ & $\Gamma_{4} \oplus M_{4}$ \\
\hline$x_{1 p}$ & 2 & 0 & 2 & 2 & 0 & 0 & 0 & 0 & -2 & -2 & 0 & 2 & 2 & 0 & 2 & 0 & 0 & 0 & -2 & 0 & $x_{1}$ \\
\hline$x_{1 n}$ & 2 & 0 & 2 & 2 & 0 & 0 & 0 & 0 & -2 & $\cdot 2$ & 0 & 2 & -2 & 0 & -2 & 3 & 0 & 0 & 2 & 0 & $x_{1}$ \\
\hline$x_{2}$ & 2 & D & 2 & -2 & 0 & 0 & 0 & 0 & -2 & 2 & 0 & 2 & $\mathbf{0}$ & 2 & 0 & -2 & 0 & 0 & 0 & 2 & $x_{2}$ \\
\hline$x_{2 x^{\prime}}$ & 2 & 0 & 2 & -2 & 0 & 0 & 0 & 0 & -2 & 2 & 0 & 2 & 0 & -2 & 0 & $\mathbf{2}$ & 0 & 0 & 0 & -2 & $X_{2}$ \\
\hline$\$ \mathbf{3}$ & 4 & 0 & -4 & 0 & 0 & 2 & 0 & -2 & 0 & $\mathbf{0}$ & 0 & 4 & 0 & 0 & 0 & 0 & 0 & 0 & 0 & $\mathbf{0}$ & $\Gamma_{5} \oplus X_{3}$ \\
\hline$\$$ & 4 & 0 & -4 & 0 & 0 & $m 2$ & 0 & 2 & 0 & 0 & 0 & 4 & 0 & 0 & 0 & 0 & 0 & 0 & 0 & 0 & $M_{3} \oplus X_{1}$ \\
\hline$\Sigma_{1 p}$ & 4 & 0 & $\mathbf{0}$ & 0 & 2 & 0 & 0 & 0 & o & 0 & -2 & -4 & 2 & -2 & -2 & $\mathbf{0}$ & 0 & 0 & 0 & 2 & $z_{1}$ \\
\hline$\varepsilon_{1 n}$ & 4 & 0 & 0 & 0 & 2 & 0 & 0 & 0 & 0 & 0 & -2 & -4 & -2 & 2 & 2 & 0 & 0 & 0 & 0 & -2 & $\Sigma_{1}$ \\
\hline$\Sigma \Sigma_{p p}$ & 4 & 0 & 0 & 0 & -2 & 0 & o & 0 & 0 & 0 & 2 & -4 & 2 & 2 & $\cdot 2$ & 0 & 0 & 0 & 0 & .2 & $\Sigma_{2}$ \\
\hline$\Sigma_{2 n}$ & 4 & 0 & 0 & 0 & -2 & 0 & 0 & 0 & 0 & 0 & 2 & -4 & -2 & -2 & 2 & 0 & 0 & 0 & 0 & 2 & $\Sigma$ \\
\hline
\end{tabular}


Table 5.8. Reduction of the twenty irreducible representations of the clusterpermutation group to the corresponding ireducible representations of the subgroup $H$ of the space group for the body-centered and face-centered cubic-lattice clusters. The dimensions of the irreducible representations of the cluster-permutation group label the columns.

\begin{tabular}{|c|c|c|c|c|c|c|c|c|c|}
\hline & 1 & 2 & 3 & 4 & 6 & 8 & 9 & 12 & 18 \\
\hline$b c c$-lattice & $\begin{array}{l}\Gamma_{1} \\
\Gamma_{1} \\
H_{1} \\
H_{1}\end{array}$ & $\Gamma_{2} \oplus H_{2}$ & & $\begin{array}{c}\Gamma_{12} \oplus H_{12} \\
\Gamma_{12} \oplus H_{12} \\
\Gamma_{1} \oplus \Gamma_{12} \oplus H_{2} \\
\Gamma_{2} \oplus H_{1} \oplus H_{12}\end{array}$ & $\begin{array}{l}N_{1} \\
N_{1} \\
N_{4} \\
N_{4}\end{array}$ & & $\begin{array}{l}\Gamma_{2 s^{\prime}} \oplus N_{2} \\
\Gamma_{2 s^{\prime}} \oplus N_{2} \\
H_{25^{\prime}} \oplus N_{3} \\
H_{25^{\prime}} \oplus N_{3}\end{array}$ & $\begin{array}{l}N_{1} \oplus N_{4} \\
N_{1} \oplus N_{4}\end{array}$ & $\Gamma_{15^{\prime}} \oplus H_{15^{\prime}} \oplus N_{2} \oplus N_{3}$ \\
\hline$f c c$-lattice & $\begin{array}{l}\Gamma_{1} \\
\Gamma_{1} \\
\Gamma_{2} \\
\Gamma_{2}\end{array}$ & $\begin{array}{l}\Gamma_{12} \\
\Gamma_{12}\end{array}$ & $\begin{array}{l}x_{1} \\
x_{1} \\
x_{2} \\
x_{2}\end{array}$ & $\begin{array}{l}L_{1} \\
L_{1} \\
L_{2} \\
L_{2}\end{array}$ & $\begin{array}{c}\Gamma_{15}^{\prime} \oplus X_{4} \\
\Gamma_{25^{\prime} \oplus X_{3}} \\
X_{5} \\
X_{5}\end{array}$ & $\begin{array}{l}L_{3} \\
L_{3}\end{array}$ & & & \\
\hline
\end{tabular}


Table 5.9. Symmetries of parameter-dependent eigenstates of the $t-t^{\prime}-J$ model that stick together in the eight-site clusters of the face-centered-cubic lattice and the square lattice. The sticking together of levels is not required by the cluster-permutation group. In the table below, $N$ denotes the number of electrons and $S$ denotes the total spin of the many-body wavefunctions. The subscript $n$ denotes representations that have a negative character for the operation $P\{E \mid 0\}$.

\begin{tabular}{cccc|cccc}
\hline & \multicolumn{3}{c}{$f c c$-lattice } & \multicolumn{4}{c}{$s q$-lastice } \\
\hline$N$ & symmetry & $S$ & $\begin{array}{c}\text { number of } \\
\text { levels }\end{array}$ & $N$ & symmetry & $s$ & $\begin{array}{c}\text { number of } \\
\text { levels }\end{array}$ \\
\hline 5 & $L_{1 n} \oplus L_{3 n}$ & $\frac{3}{2}$ & 2 & 4 & $\phi_{1} \oplus \phi_{3} \oplus \phi_{4}$ & 2 & 2 \\
5 & $L_{1 n} \oplus L_{3 n}$ & $\frac{1}{2}$ & 2 & 6 & $\Gamma_{1 n} \oplus M_{1 n}$ & 2 & 2 \\
6 & $L_{1 n} \oplus L_{3 n}$ & 1 & 4 & 7 & $\Gamma_{1 n} \oplus M_{1 n}$ & $\frac{3}{2}$ & 2 \\
7 & $L_{1 n} \oplus L_{3 n}$ & $\frac{3}{2}$ & 2 & & & & \\
7 & $L_{1 n} \oplus L_{3 n}$ & $\frac{1}{2}$ & 2 & & & & \\
\hline
\end{tabular}




\section{V.9 Figures for Chapter V}

Figure 5.1. Four-, eight-, and sixteen-site clusters with periorlic boundary conditions in the square lattice. The $\sqrt{M} \times \sqrt{M}$ tilings of the square lattice are highlighted in gray. The four-site cluster (a) is a self-contained cluster since the periodic boundary conditions do not add any new lattice connections. This fact is highlighted by the dashed lines in (a). The eight-site cluster (b) is not a 1N:-determined lattice, as discussed in the text, and lies in the high-symmetry regine. The sixteen-site cluster (c) has no additional symmetry for arbitrary interactions but does possess hidden symmetry for Hamiltonians that contain only $1 \mathrm{NN}$ interactions.

a)

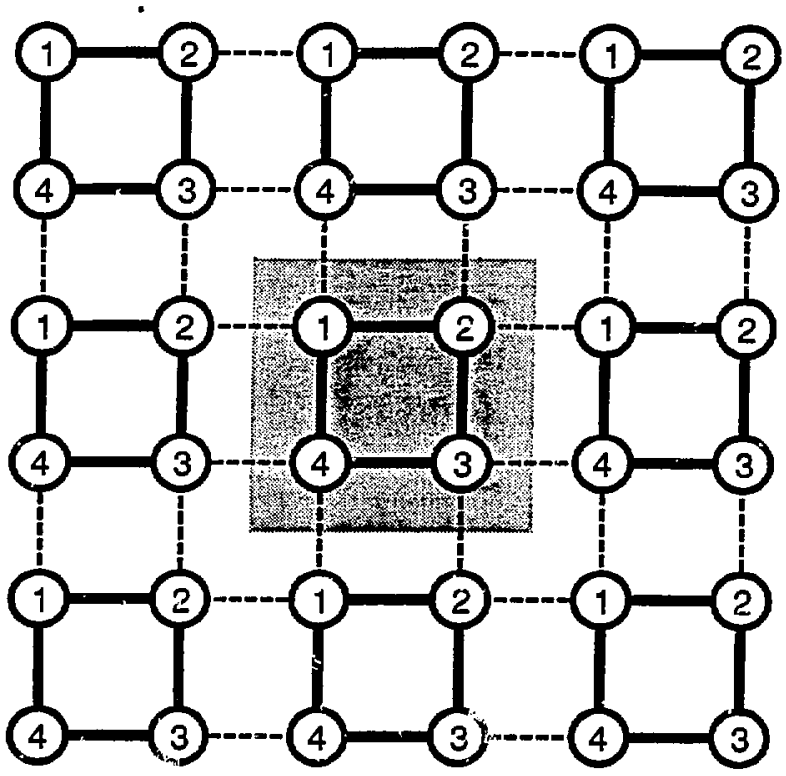


b)

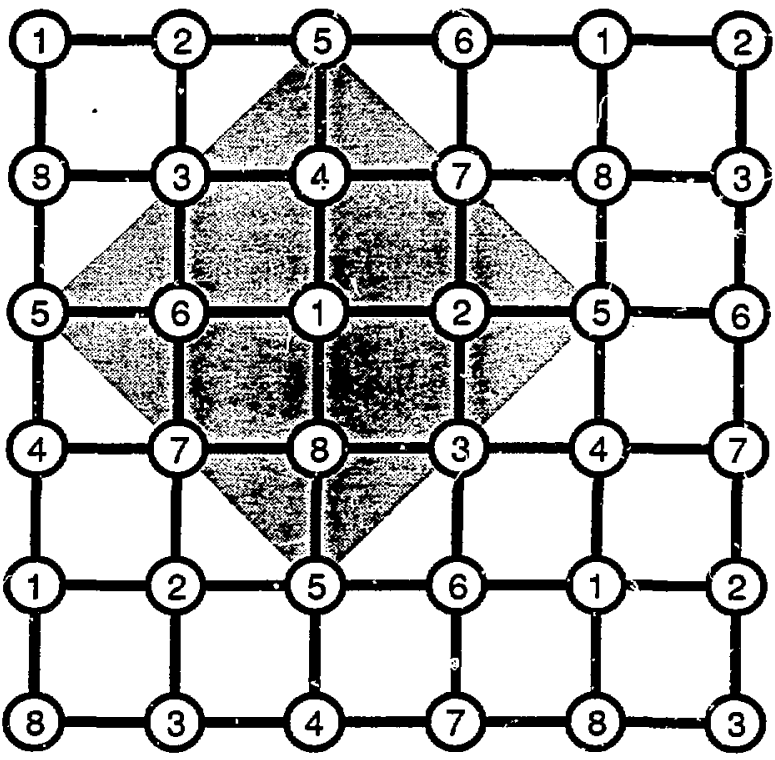


c)

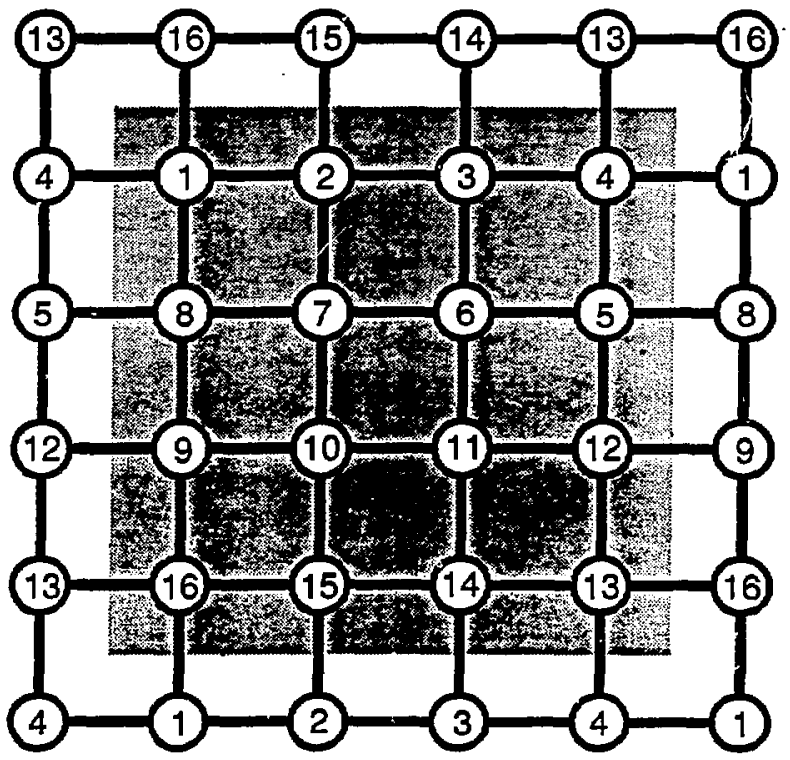

